\title{
Corneal desiccation in rigid gas permeable contact lens wear : time to deal with 3- and 9-o clock staining
}

Citation for published version (APA):

van der Worp, E. C. J. (2008). Corneal desiccation in rigid gas permeable contact lens wear : time to deal with 3- and 9-o clock staining. [Doctoral Thesis, Maastricht University]. Datawyse / Universitaire Pers Maastricht. https://doi.org/10.26481/dis.20081128ew

Document status and date:

Published: 01/01/2008

DOI:

10.26481/dis.20081128ew

Document Version:

Publisher's PDF, also known as Version of record

\section{Please check the document version of this publication:}

- A submitted manuscript is the version of the article upon submission and before peer-review. There can be important differences between the submitted version and the official published version of record.

People interested in the research are advised to contact the author for the final version of the publication, or visit the DOI to the publisher's website.

- The final author version and the galley proof are versions of the publication after peer review.

- The final published version features the final layout of the paper including the volume, issue and page numbers.

Link to publication

\footnotetext{
General rights rights.

- You may freely distribute the URL identifying the publication in the public portal. please follow below link for the End User Agreement:

www.umlib.nl/taverne-license

Take down policy

If you believe that this document breaches copyright please contact us at:

repository@maastrichtuniversity.nl

providing details and we will investigate your claim.
}

Copyright and moral rights for the publications made accessible in the public portal are retained by the authors and/or other copyright owners and it is a condition of accessing publications that users recognise and abide by the legal requirements associated with these

- Users may download and print one copy of any publication from the public portal for the purpose of private study or research.

- You may not further distribute the material or use it for any profit-making activity or commercial gain

If the publication is distributed under the terms of Article $25 \mathrm{fa}$ of the Dutch Copyright Act, indicated by the "Taverne" license above, 


\section{Corneal Desiccation in Rigid Gas Permeable Contact Lens Wear}

Time to deal with 3- and 9-o'clock staining

Eef van der Worp 
Eef van der Worp

Corneal Desiccation in Rigid Gas Permeable Contact Lens Wear

Time to deal with 3- and 9-o'clock staining

(C) Copyright ECJ van der Worp 2008

All rights reserved. No part of this book may be translated or reproduced in any form by print, photo print, microfilm, or any other means without prior written permission of the publisher.

ISBN 9789052787732

Vormgeving: Datawyse bv

Druk: Datawyse | Universitaire Pers Maastricht 


\section{Corneal Desiccation in Rigid Gas Permeable Contact Lens Wear}

\section{Time to deal with 3- and 9-o'clock staining}

\section{Proefschrift}

ter verkrijging van de graad van doctor

aan de Universiteit Maastricht

op gezag van de Rector Magnificus

Prof. mr G.P.M.F. Mols

volgens het besluit van het College van Decanen

in het openbaar te verdedigen

op vrijdag 28 november 2008 om 10.00 uur

door

Everardus Cornelis Johannes van der Worp

Geboren op 3 augustus 1969 te Schalkwijk (Houten)

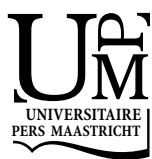




\section{Promotor}

Prof. dr. F. Hendrikse

\section{Copromotores}

Dr. J. de Brabander

Dr. H. Swarbrick (University of New South Wales, Sydney, Australië)

\section{Beoordelingscommissie}

Prof. dr. ir. L.H. Koole (voorzitter)

Prof. dr. A. Kijlstra (Universiteit van Amsterdam)

Prof. dr. F. Ramaekers

Prof. dr. G.H.M.B van Rens (Vrije Universiteit, Amsterdam)

Dr. A.J.P. Rouwen (Centraal Militair Hospitaal, Utrecht) 
"Tijd heelt vele wonden..."

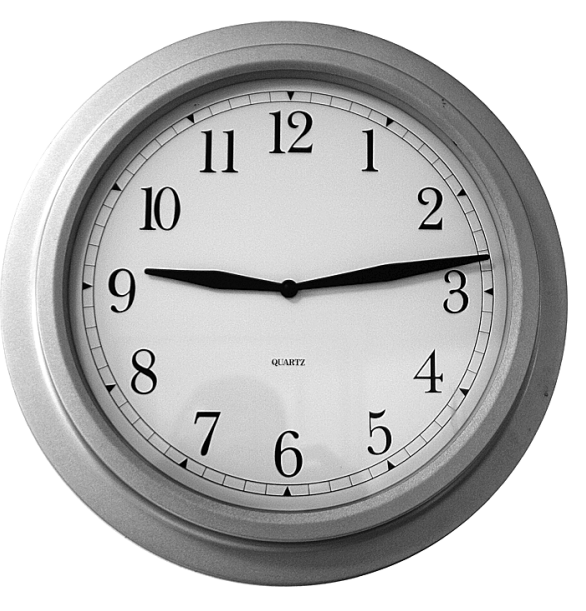





\section{Contents}

\section{CHAPTER 1}

Introduction $\quad 9$

Introduction to the studies $\quad 11$

Aims and outline of this thesis $\quad 34$

\section{CHAPTER 2}

The art of rigid gas permeable contact lens fitting 43

2.1 Optimising RGP lens fitting in normal eyes using 3D topographic data 45

2.2 Respecting the shape of the cornea in rigid gas permeable lens fitting 55

\section{CHAPTER 3}

Background \& classification of corneal desiccation in rigid gas permeable contact lens wear

3.1 Corneal desiccation in rigid contact lens wear:

3- and 9-o'clock staining - a review

3.2 The development of an image grading scale with high linearity for 3- and 9-o'clock staining

\section{CHAPTER 4}

Clinical Studies: Evaluation of 3- and 9-o'clock staining in existing rigid gas permeable contact lens wearers

4.1 Evaluation of signs and symptoms in 3- and 9-o'clock staining 115

4.2 Analysis of contact lens related variables in 3- and 9-o'clock staining

\section{CHAPTER 5}

Tear volume and eyeblinking in relation to 3- and 9-o'clock staining

5.1 Tear meniscus height evaluation in rigid gas permeable lens wear

5.2 Eyeblink frequency and type in relation to 3- and 9-o'clock staining and gas permeable contact lens variables

\section{CHAPTER 6}

Summary and Conclusions $\quad 185$

$\begin{array}{ll}\text { Samenvatting en Conclusies } & 197\end{array}$

Abbreviations \& Terms $\quad 209$

Dankwoord 211

$\begin{array}{ll}\text { Curriculum Vitae } & 215\end{array}$

$\begin{array}{ll}\text { Publications } & 217\end{array}$ 



\section{CHAPTER 1 Introduction}


10 CHAPTER 1 


\section{History and Usage of Contact Lenses}

Contact lenses, originally designed to correct severe optical irregularities of the human cornea, are widely used today to correct ametropia in non-pathological eyes. The basic idea of putting a refractive device directly on the eye has its origin in the early 16th century from the work of Leonardo da Vinci ${ }^{1}$ (1508) and later Descartes $^{2}$ (1637), but it was not until 1887 that Saemisch and Müller in Germany used a lens that was developed for use on a human eye. ${ }^{3}$ The term contact lens (first used as "Kontaktbrille" in the German language, meaning contact-glasses) was introduced by Fick in $1888 .{ }^{4}$ The first lenses were large diameter glass shells. ${ }^{5,6}$ These lenses actually did not contact the cornea, but rather bridged over it while the periphery rested on the scleral portion of the eye. The wearing time of the original glass shell lenses was limited to a few hours because of the lack of tear film exchange behind the lens and because of oxygen shortage to the cornea.

Smaller versions of the original contact lens were developed that were placed directly on the cornea, and the term "corneal contact lens" was introduced. Technically these lenses do not touch the cornea either because a tear film is present between the lens and the cornea. In the mid 1930s a plastic material (Poly Methyl Methacrylate, PMMA) was used for the production of these lenses, ${ }^{7}$ and the PMMA rigid contact lenses were, and still are, referred to as hard lenses. The usage of contact lenses increased and the lenses became more widely available. Oxygen delivery to the cornea with these hard lenses is primarily provided through tear film exchange underneath the lens because the material itself is not gas permeable. It is estimated that the tear pump provides about one third of the oxygen requirements of the cornea in daily wear (DW) use of hard contact lenses. ${ }^{8,9}$ Hence, on average a relative shortage of oxygen delivery to the cornea can be expected with these type of lenses. ${ }^{10}$

In 1978, the first rigid lens material for contact lens wear was approved by the Food and Drug Administration (FDA) in the United States of America (USA). Since then, materials with various levels of oxygen permeability have been developed, allowing oxygen transmissibility to be adequate enough for normal corneal functioning in daily and extended wear (EW) use of these lenses. ${ }^{11}$ These lenses are referred to as rigid gas permeable contact lenses, abbreviated as RGP lenses or, in large parts of the world (including in the USA), as GP lenses. Apart from material improvements, rigid lenses have further advanced with the introduction of aspheric back surface geometries. This concept was introduced by Hirst. ${ }^{12}$ These lenses have the ability to follow the shape of the cornea more closely, which is believed to promote comfortable lens wear. ${ }^{13}$ 
In the meantime, the FDA approved a new contact lens modality (1971) originally designed by Wichterle ${ }^{7}$ using a poly-hydroxyethyl methacrylate (HEMA) material, which was used to produce the so called soft or hydrogel contact lenses. A new era started with the introduction of these hydrated lenses, which typically had a water content of $38 \%$. Subsequent material improvements resulted in higher water content (up to $80 \%$ ). Higher water content materials (given the same thickness) improve the oxygen permeability of the lens. This is considered essential because the tear film exchange underneath hydrogel lenses is suggested to be low; a tear replenishment underneath the lens of only 1 to $2 \%$ has been suggested, ${ }^{14}$ which means that the bulk of the desired oxygen needs to go through the lens rather than relying on the tear pump. The water content and the oxygen permeability (Dk) of a soft lens have a linear relationship. By making the lens thinner, manufacturers tried to improve the oxygen transmissibility (the oxygen permeability of a lens in relation to the thickness of the lens, $\mathrm{Dk} / \mathrm{t}$ ). However, there are limits to the water content and minimal thickness of a soft contact lens. Handling issues (not enough stability) and breakability become problematic with very high water content, thin lenses.

Silicone materials have always been looked upon as a possible way to overcome this status-quo with conventional soft lenses. Silicone materials have long been known for their reversed linear relationship between oxygen permeability and water content: the lower the water content, the higher the oxygen permeability. However, a major disadvantage of using this material on the human eye is the hydrophobic nature of the material, ${ }^{15}$ which prevents comfortable lens wear in adults. The tear composition in newborn children appears to be different, and wettability is usually not a problem. This makes silicone a popular choice of material for aphakic EW contact lenses in young children. ${ }^{16}$ It was not until 1999 that silicone hydrogel contact lenses entered the market. ${ }^{17}$ Many silicone hydrogel materials are available now, and one way or the other they have been specially treated to make the surface of the lens hydrophilic in nature. Originally designed for day and night use because of the exceptionally high oxygen transmissibility of the lens, ${ }^{18}$ it is now used worldwide as a popular lens of choice for DW as well. One in every two soft lens fits for DW in the USA today is with a silicone hydrogel material. ${ }^{19}$

There are significant differences among countries in contact lens usage and in mode of correction. Taking all lens modalities into account, it appears that Japan $(15 \%)$ and the Nordic countries (Denmark, Finland, Norway, Sweden combined) with $14 \%$ have the highest rates of contact lens wearers globally. ${ }^{20}$ In the Netherlands, contact lenses are a relatively popular mode of wear compared to most other countries; $11.9 \%$ of the Dutch population above 12 years of age wears contact lenses according to a recent survey, ${ }^{21}$ which appears to be close to the penetration 
of contact lens wear in the biggest single contact lens market in the world (USA) with $12 \%$. In most European countries the contact lens penetration rates are much lower: France and Germany have percentages in the range of $4.5 \%$, while the United Kingdom is slightly higher at $6.2 \%{ }^{20}$

\section{The Classic Debate: Soft Lenses versus Rigid Gas Permeable Lenses}

When fitting contact lenses, the classic consideration always has been whether to use rigid gas permeable lenses or soft lenses for new wearers. When all the positive characteristics that modern rigid gas permeable lenses have are listed, one could easily be misled and conclude that rigid gas permeable lenses must be the most popular type of lenses prescribed in the world. One of the most important advantages of rigid gas permeable lenses is superior optical quality, and another benefit is the superb oxygen transmissibility of today's available rigid gas permeable lenses. But the reality is that currently less than 1 out of every 10 lens fits around the world is with rigid gas permeable lenses, ${ }^{22}$ although large variations exist in different countries as indicated in figure 1 . In the Netherlands, $36 \%$ of the contact lens population is wearing rigid lenses, ${ }^{21}$ and $24 \%$ of new lens fits and refits are with the rigid gas permeable lens modality. ${ }^{22}$ In some countries, including the UK and Japan, the number of rigid gas permeable refits is remarkably higher than the number of new lens fits (fig. 1). This seems to imply that rigid gas permeable lenses may serve as a secondary option if other methods fail or are less successful.

In summary: while the majority of lens fits globally is with soft lenses, substantial differences in the ratio of soft versus rigid gas permeable lenses are present, and average values do not do justice to local markets. ${ }^{22}$ Considerations related to choosing one lens modality over the other will be discussed in the next paragraphs. 


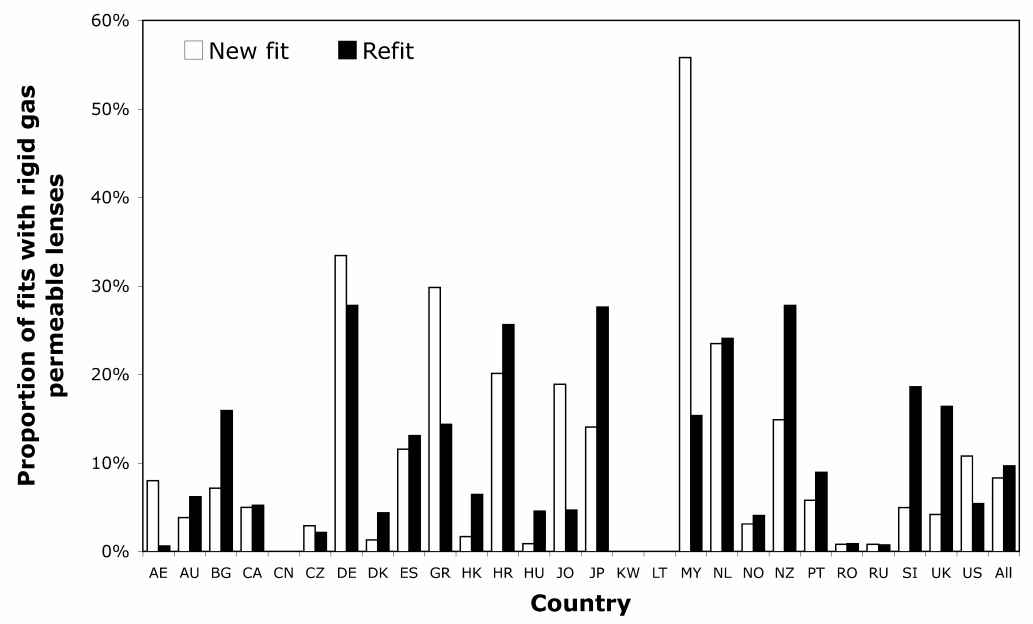

FIGURE 1. Percentage of rigid gas permeable lens fits in 2007 as a proportion of total lens fits in United Arab Emirates (AE), Australia (AU), Bulgaria (BG), Canada (CA), China (CN), Czech Republic (CZ), Germany (DE), Denmark (DK), Spain (ES), Greece (GR), Hong Kong (HK), Croatia (HR), Hungary (HU), Jordan (JO), Japan (JP), Kuwait (KW), Lithuania (LT), Malaysia (MY), the Netherlands (NL), Norway (NO), New Zealand (NZ), Portugal (PT), Romania (RO), Russia (RU), Slovenia (SI), the United Kingdom (UK) and the United States (US). New fits (white bars) and refits (black bars). ${ }^{22}$

\section{Quality of Vision}

If superb optical quality is desired, rigid gas permeable lenses generally provide the best visual outcome. Rigid gas permeable lenses are the only optical correction method that is able to correct the irregular cornea. Mild forms of corneal irregularities can usually be corrected with standard rigid gas permeable lenses. For more irregular corneal surfaces such as in keratoconus, post corneal grafts, high astigmatism and post-refractive surgery, specially designed rigid gas permeable contact lenses can be fitted which are usually the only method that can provide acceptable visual acuity. Higher order optical aberrations can often be corrected by using rigid gas permeable lenses. ${ }^{23-25}$ Soft lenses do not correct these aberrations since they follow the shape of the anterior ocular surface almost entirely. Laser refractive surgery actually induces large amounts of higher order aberrations (in particular spherical aberration when correcting myopia) due to the nature of the procedure..$^{26,27}$

The discussion on the visual quality of different correction methods can be extended to the area of presbyopia. At this moment about half of the population in the developed world has reached the presbyopic age. ${ }^{28}$ The contact lens wearers within this group are especially interested in a solution for their decrease in reading 
abilities that does not require the use of glasses, which is what they are used to. Although many soft bifocal systems are available today, it is generally agreed upon that soft bifocals have their limitations, especially when higher reading additions are required. The same is true for refractive surgery: presbyopic correction options are limited. Rigid gas permeable bifocal lenses are currently considered to be the best mode of vision correction as an alternative to glasses, mostly because both alternating and simultaneous rigid gas permeable bifocal lenses have the ability to translate on the eye, providing the most optimal vision possible for far and near. ${ }^{29}$

\section{Corneal Physiology}

Another consideration for soft versus rigid gas permeable lenses is ocular health. The high safety levels of rigid gas permeable lenses relative to other contact lens modalities have been confirmed in the past by many researchers. ${ }^{30-33}$ Work by Cavanagh ${ }^{34,35}$ has revealed that compared to soft lenses, rigid gas permeable lenses demonstrate lower levels of bacterial binding to the corneal epithelium, even if compared to high oxygen transmissible silicone hydrogel lenses. In fact, rigid gas permeable lenses showed no statistically significant difference in bacterial binding compared to a control group not wearing lenses at all.

A large study looking at the annual incidence rates (cases per 10,000 wearers) and analyzing over 55,000 contact lens wearers looked at severe and non-severe cases of keratitis for different wearing modalities and lens types. It was found that wearers of some modes of contact lens wear have a higher risk of developing adverse reactions (table 1). ${ }^{36-38}$ The lowest incidence rates were found in rigid gas permeable lens wear, both within the DW and the EW groups (although EW rigid gas permeable wear is rare, and was limited to only 18 wearers in this study).

TABLE 1. The annual incidences (cases per 10,000 wearers) of keratitis with different types of contact lenses ${ }^{38}$

\begin{tabular}{lcc}
\hline & non-severe keratitis & severe keratitis \\
\hline DW & 5.7 & \\
Rigid gas permeable & 9.1 & 4.9 \\
Soft - daily disposables & 14.1 & 6.9 \\
Soft - non-daily disposables & 55.9 & 0.0 \\
Silicone hydrogel soft & & \\
EW & 0.0 & 0.0 \\
Rigid gas permeable & 48.2 & 96.4 \\
Soft & 98.8 & 19.8 \\
Silicone hydrogel soft & &
\end{tabular}


Another advantage of rigid lenses over soft lenses is deposit resistance. Rigid gas permeable lenses are less prone to deposition compared to soft lenses, although there is a high material variability. ${ }^{39}$ Also, less solution allergies and toxic reactions have been reported in rigid gas permeable lens wear than in soft lens wear, and contact lens induced papillary conjunctivitis is believed to be one of the complications less frequently seen in rigid gas permeable lens wear than in soft lens wear. ${ }^{39,40}$

Generally speaking, when it comes to health of the eye, rigid gas permeable lenses are believed to be the safest alternative to glasses for the correction of ametropia. One of the few complications that is typical for, and exclusively seen in, rigid gas permeable lens wear is 3-and 9-o'clock staining, which is the focus of this thesis.

\section{Comfort}

Another issue regarding successful contact lens wear is lens comfort. Comfort is considered the main reason for the greater popularity of soft lenses as opposed to rigid gas permeable lenses. ${ }^{41-43}$ This despite the fact that some investigators have found that comfort is relative in nature, and that the main obstacle is initial comfort (not the long term comfort). Fonn et al. ${ }^{42}$ found that after three months of lens wear, comfort in soft and rigid gas permeable lens wear in unadapted contact lens wearers was not significantly different. Vajdic et al. ${ }^{44}$ in a prospective study of adapted soft and rigid contact lens wearers found similar types and frequency of ocular symptoms. The use of a topical anaesthetic at the fitting and dispensing of rigid gas permeable lenses has been suggested to overcome the initial comfort issues and has been shown to result in a decrease in dropouts. ${ }^{45}$

Still, comfort remains the most important issue in the classic consideration of soft versus rigid gas permeable lenses. While innovations in soft lens wear have been fairly radical over the recent years, including more frequent replacement of lenses (up to the point of daily replacement) and the introduction of silicone hydrogel materials, rigid gas permeable lenses have not undergone substantial improvements in lens design, material or frequency of wear in the recent years. More so, contact lens practitioners are still using the same basic techniques for fitting rigid gas permeable lenses as were used in the PMMA era in the 1950s. This typically means they are relying on two centrally measured keratometry values and are using trial contact lens sets for the fitting of these lenses. These trial sets have major limitations because lens design is fixed, and the trial lens sets typically contain lenses with 0.05 to $0.10 \mathrm{~mm}$ differences in back optic zone radius (BOZR) between sequential lenses, which are relatively large steps. This does not always support the cornea to the fullest and this may compromise comfort (see part 2.1 of this thesis). New sophisticated and ultra-precise lathe manufacturing is available today that can pro- 
duce lenses of any desired shape with a very high accuracy. Also, more precise techniques to measure the cornea and its shape are available to practitioners, potentially improving comfort of lens wear.

\section{Measuring the Shape of the Cornea}

Eye care practitioners who fit contact lenses have always had great interest in understanding the shape of the cornea, rigid gas permeable lenses in particular. With the known shape, contact lenses can be fitted that optimize the cornea-lens relationship as much as possible. Generally, mimicking the shape of the cornea promotes wearing comfort and can reduce mechanical effects of the lens on the cornea, thus potentially preventing unwanted corneal changes (part 2.2).

\section{Keratometry}

The standard procedure in contact lens practice has been (and still is) to measure the cornea with a keratometer (derived from "keratos," the Greek word for cornea). Keratometry measures typically the average curve of the central three millimeters of the cornea in two meridians. This three millimeter area is only a small portion of the corneal surface (of which the total size is $11.8 \mathrm{~mm}$ in diameter on average). Measuring the central three millimeters covers only about $8 \%$ of the total corneal surface in an average cornea. Contact lenses stretch out over a much larger part of the cornea than this, and keratometry does not provide any information about the peripheral corneal shape. Theoretically, peripheral corneal curves can be measured with a keratometer by having the subject look at an angle of 25 or 30 degrees nasally, temporally, superiorly and inferiorly and performing keratometry under these angles. ${ }^{46}$ If this information is related to the central curves of the cornea, some idea about the amount of flattening towards the periphery can be obtained. Apart from the fact that with a keratometer it is hard to obtain reliable peripheral curve data from the periphery of the cornea, computation of corneal shape from this data is difficult because two different measurements are used (sagittal versus tangential radii). Also, a keratometer measures curves, and curves are not the equivalent of shape. More and more, contact lens practitioners rely on total corneal shape information rather than on corneal curve data alone. This is crucial when dealing with refractive surgery, orthokeratology and keratoconus but also for the design and manufacturing of any type of contact lens.

\section{Corneal Topography}

Corneal topographers can instantly provide information about thousands of data points on the corneal surface and will give us a better understanding of corneal 
shape than will keratometry. Corneal topographers can be categorized into reflection systems and height systems. ${ }^{47}$

\section{Reflection Systems}

Most topographers, especially those used in contact lens practices, are reflection systems. For centuries, eye care practitioners have been using the reflection capacity of the anterior surface of the eye to obtain a qualitative impression of the integrity of the cornea. Historically it has been described to diagnose corneal integrity by viewing the reflected image of a rectangle window. This simple diagnostic tool is based on the fact that the boundary air-tear film acts as a mirror. From this the basis for quantitative corneal topography was described by Helmholtz, Placido and Gullstrand in the late 19th century. ${ }^{48-50}$

Instead of pairs of objects, as used with keratometry, Placido ${ }^{50}$ in 1880 used a disc with concentric rings and a central hole through which he observed the image reflected by the subject's eye. Strictly speaking, the name keratoscopy means viewing the cornea. So, the original Placido disc is a true keratoscope in the hands of the practitioner looking at the formed image. With photographing of the image, the name of the device has historically been changed in photokeratoscope. With the replacement of the photo camera by a CCD (charge-coupled device) camera, the name of the instrument became videokeratoscope. After the implementation of computer assisted software algorithms to analyze the corneal pictures, the name was changed to corneal topography.

One of the main disadvantages of a reflection system topographer is the limited area that can be measured, although it is a much larger area than the keratometer can measure. Often the measurable area is limited to six or seven millimeters of the corneal surface. In order to measure a larger surface area, it is important to minimize upper eyelid and nose interference, which can cause shadows on the cornea and result in missing data points. ${ }^{51}$ The more irregular a cornea is, the more peripheral data points may be missing. Reflected peripheral rings may start to overlap, and the topographer does not know which ring to analyze. This effect is described as 'ring jam'. Possibly, newly developed color coded corneal reflection topographers may be able to alleviate this problem..$^{52}$

Another disadvantage of reflection systems is that it is crucial to have a well wetting ocular surface. Taking several measurements to exclude artifacts caused by dry spots on the cornea is strongly advised when using reflection systems. Having the subject blink a number of times before measurement could help in achieving this. If this does not provide a well wetting ocular surface, tear supplements can be used 
to overcome the problem. Dynamic corneal topography in which a 'live' corneal topography image is generated, currently an experimental technique, could potentially help overcome this limitation. ${ }^{53}$

\section{Height Systems}

Appreciating the disadvantages of reflection systems, alternatives to the Placido disc based corneal topographers have been developed, ${ }^{54-56}$ broadly categorized as height systems. One of the main disadvantages of reflection systems is that they measure corneal curvature and not corneal height. Height maps can be generated from curvature data, using fast and smart algorithms combined with logical iterative interpolation and extrapolation. These can be reasonably accurate, given a reliable starting point in the Placido disc image and taking into account some assumptions about the shape of the cornea.

But height systems generate sagittal height data directly from the cornea. Some systems use a scanning slit or a Scheimflug image, such as in Orbscan ${ }^{\circledR}$ (Bausch \& Lomb), Pentacam (Oculus Inc.) and Galilei ${ }^{\mathrm{Tm}}$ (Ziemer Ophthalmic) systems. The principle of such devices can be easily appreciated from a cross section view of the cornea. In the slit image the profile of the front and back corneal surface can be seen. To overcome distortion by different incidence angles, a Scheimpflug correction system is built in. ${ }^{54}$

Analysis of a height map is different from analysis of a curvature map. A height map representing the total, absolute sagittal height of the cornea would in fact not provide much detail at all. It would only show the obvious fact that the central cornea is higher (or more forward) than the periphery of the cornea. Height maps only become useful after matching the corneal surface with a so-called 'best fit sphere' (or sometimes 'a best fit ellipse'). Everything that is higher (closer to the observer) is color-coded with warmer colors; everything that is positioned further away from the observer compared to the best fit sphere or ellipse is presented with cooler colors. This way, it is immediately appreciated what the actual shape of the cornea is.

More experimental in nature for corneal imaging are instruments such as anterior segment optical coherence tomography (OCT) and interferometry (Maastricht Shape Topographer, MST). These systems are able to present a height map of the total anterior ocular surface area, including the limbus and parts of the sclera.

The MST, ${ }^{55}$ which was used for one of the studies in this thesis (part 2.1) uses Fourier profilometry. It projects a line pattern from two different directions onto 
the front surface of the eye in which fluorescein acts as a diffusing medium. Because the line patterns are viewed by a central camera, they become curved in the image depending on the shape of the eye. Fourier analysis can transform this information to height data. From this it is possible to create cross sections of the cornea and the sclera. As with Placido disc devices, a good image is essential. Advantages of the MST are that it directly measures shape of the entire front surface of the eye, although fluorescein is needed to form a 'screen' to project the images on.

More recently, $\mathrm{OCT}^{57}$ was developed as a method to image the anterior segment of the eye. Instead of sound waves (as with ultrasound techniques), OCT uses light waves that are sent through the cornea. The principle behind this device is a Michelson interferometer in which time differences using two imaging paths from the same target are used to compute distance data. From these two images, height information from the anterior ocular surface can be obtained, including the limbal area (the sulcus) and a small portion of the sclera (Figure 2).

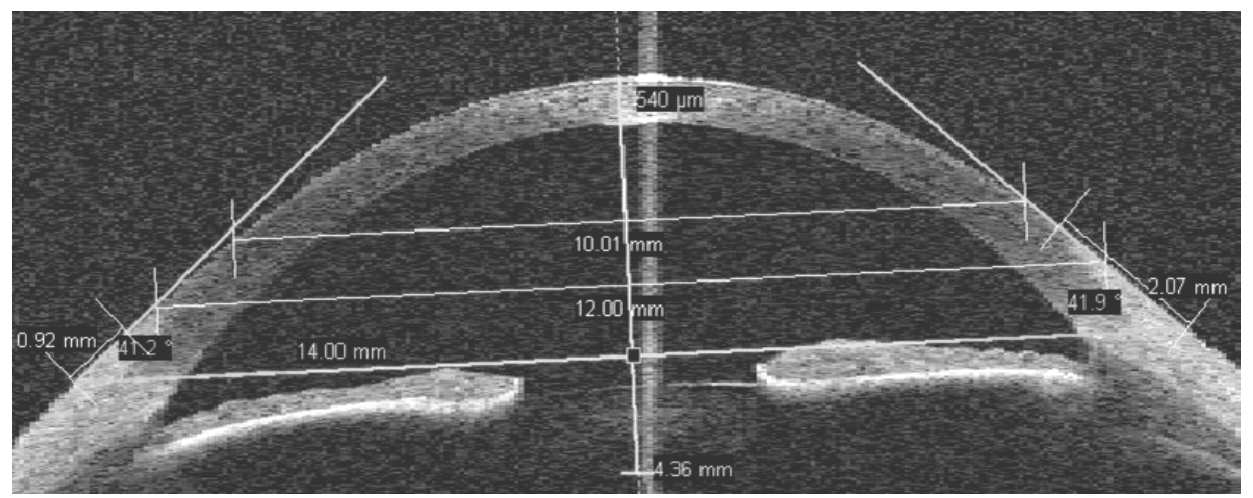

FIGURE 2. Design of a semi-scleral lens based on anterior segment OCT data (Visante ${ }^{\mathrm{TM}}$, Zeiss).

In summary: although current corneal topographers have some limitations, the accuracy of lens fitting can be improved using the thousands of data points per cornea that these instruments can generate compared to using the limited keratometry data, typically reduced to two data points.

\section{Corneal Desiccation in Contact Lens Wear}

With currently available corneal analyzing techniques, fitting methods, lens designs and materials, the majority of contact lenses can be fitted and worn successfully. One of the most frequently reported remaining complaints, and a main reason why patients drop out of lens wear, relates to dryness symptoms, ${ }^{58-61}$ possibly affecting 
up to $50 \%$ of all contact lens wearers. ${ }^{62}$ This occurs in both soft and rigid gas permeable lens wear but seems to be different in manifestation, etiology and severity. In soft lens wear, corneal desiccation manifests as dehydration staining while in rigid gas permeable lens wear, desiccation may lead to 3- and 9-o'clock corneal staining.

\section{Dehydration Staining}

Corneal staining as a result of lens dehydration in soft lens wear is a common finding. Dehydration staining can lead to discomfort, bulbar hyperemia, decreased visual acuity and discontinuation of lens wear. It presents typically in the midperipheral, inferior part of the cornea and parallel to the limbus. ${ }^{63,64}$ Because of its location and curved shape it is often referred to as 'smile staining. ${ }^{65,66}$ Several researchers have suggested depletion of the post lens tear film arising from lens dehydration as a mechanism behind corneal staining, hence the term dehydration staining. ${ }^{67-69}$ In dry eye conditions, pervaporation of water through the lens occurs and water is ultimately subtracted from the post lens tear film. At this point the post lens tear film becomes very thin or vanishes. The lens can come in contact with the epithelium and cause staining as a result of water withdrawal from the epithelial cells or by mechanical pressure on the epithelial cells. ${ }^{65,70}$

Material properties and lens thickness seem to play an important role in the occurrence of dehydration staining. ${ }^{59,68,71-74}$ Thin, high water contact lenses have been reported to have the highest risk of developing dehydration staining. ${ }^{66-68}$ Prevalence has been reported to be as high as $100 \%$ in ultra thin, high water content lenses, of which $70 \%$ was reported to be clinically relevant. ${ }^{67,68}$ But the water bounding properties of the material have recently gained a lot of attention as well and seem to be one of the key factors in preventing dehydration staining: materials with better water bounding properties have proven to be beneficial in alleviating the dryness symptoms. ${ }^{75-79}$

\section{3- and 9-O'Clock Staining}

While corneal desiccation in soft lens wear has gained a lot of attention over the recent years, corneal desiccation in rigid gas permeable lens wear has not been widely investigated in the recent past, ${ }^{14}$ and in general very few articles into the therapeutic options for 3- and 9-o'clock staining meet the criteria of randomized clinical studies. ${ }^{80}$ The most recent papers on this subject date from the 1994-1997 period, with peaks of interest in the late 1960s, late 1980s and early 1990s. The oldest paper found on 3- and 9-o'clock staining was written by Steele in 1959. ${ }^{81}$ Subjective symptoms of 3- and 9-o'clock staining typically include discomfort, foreign body sensation, burning, reduced wearing times and photophobia. ${ }^{4,28}$ When 
these symptoms occur they have been reported to increase during the day ${ }^{28}$ However, subjective symptoms of ocular irritation seem not necessarily to correlate with the objective findings. Low grades and even absence of discomfort in 3- and 9o'clock staining have been reported. ${ }^{2,15,46}$

With regards to subjective observations: bulbar hyperemia has been described as a first sign in 3- and 9-o'clock staining, ${ }^{2}$ and it appears a very common sign in the more advanced stages of the condition. ${ }^{43}$ Anecdotal clinical observation suggests that patients suffering from 3- and 9-o'clock staining may present with higher levels of conjunctival fluorescein staining, but to the best of our knowledge the occurrence of conjunctival staining in patients with 3- and 9-o'clock staining has not been investigated before. In part 4.1 of this thesis, the possible relationship between conjunctival staining and 3- and 9-o'clock staining will be further considered.

The term 3- and 9-o'clock staining, ${ }^{4,27-29}$ also referred to as peripheral corneal staining, ${ }^{18,21,22}$ peripheral corneal desiccation, ${ }^{17,23,24-27}$ 4-8 o'clock staining, ${ }^{31}$ juxtaposition staining ${ }^{32}$ or persistent nasal and temporal stippling ${ }^{33}$, describes a condition of corneal epithelial staining in the limbal area at the two to five o'clock and seven to ten o'clock positions of the cornea. The amount of staining in 3- and 9-o'clock staining varies, ranging from transient superficial epithelial stippling to an intense staining defect with localized thinning of the cornea (dellen formation), possibly leading to a vascularized limbal keratitis. ${ }^{82}$ The etiology of the condition and potential treatment options is not agreed upon in the international literature. These potential mechanisms behind and the possible treatment options for the condition will be discussed in part 3.1 of this thesis.

This thesis will focus in depth on the commonly seen and often frustrating condition in rigid gas permeable lens wear that will be referred to by the clinical sign that marks the corneal condition: 3- and 9-o'clock staining. This simply describes the objective manifestation of the condition, without implying a possible etiology.

\section{The Corneal Epithelium}

The corneal epithelium is the outermost layer of the five layer corneal structure, and it forms the outer barrier function. Beneath the epithelium, the anterior limiting membrane (Bowman's membrane) separates the epithelium from the stromal layer. The stroma forms the bulk of the cornea. On the other side of the stroma, the posterior limiting membrane (Descemet's membrane) separates the stroma from a single cell layer that forms the inner barrier, the corneal endothelium. 
The outer layer of the cornea, the epithelium, measures approximately $50.6 \pm 3.9$ $\mu \mathrm{m}$ in thickness. ${ }^{83}$ Three distinct cell shapes are recognized within the epithelial layer: basal, intermediate (wing) and superficial (squamous) cells. Basal cells are small (approximately $10 \mu \mathrm{m}$ in diameter), while wing cells are larger, typically 30 $45 \mu \mathrm{m}$ in diameter, and superficial cells measure the largest surface area: they are roughly $40-50 \mu \mathrm{m}$ in diameter. ${ }^{84}$ When corneal desiccation staining is present, it typically involves the superficial epithelial cells, possibly the intermediate cells and seldom the deeper basal cells. It is very unlikely that deeper corneal layers such as the anterior limiting membrane or the stroma are affected. The corneal nerves are located in the deep basal layer of the epithelium and/or underneath that, and are typically not affected in low to moderate levels of corneal desiccation staining.

The lifecycle of an epithelial cell has been estimated to be around seven days. ${ }^{85}$ During this period it evolves from a basal cell to a superficial cell, after which it ceases and is sloughed off at the ocular surface. The movement of epithelial cell proliferation within the epithelial layer is believed to be accomplished by a combination of processes. One movement is forced by cell division of the basal cells at the basement layer of the epithelium. Then cells start to move outwards towards the corneal surface area (the x movement), forced by new cells that are produced at the basal cell level. At the same time, cell movement from the corneal limbal area towards the center of the cornea takes place (the y movement); studies have shown that the far periphery of the cornea has a higher epithelial mitotic rate than the central cornea does ${ }^{86}$ forcing cells to move towards the center. The combination of the $\mathrm{x}$ and the $\mathrm{y}$ movement results in a diagonal vector movement (the $\mathrm{z}$ movement) of epithelial cells towards the center of the cornea and the corneal surface. Once cells reach the ocular surface and die, they desquamate into the tear film layer and are disposed of via the tear drain system.

It appears that in a normal cornea, peripheral epithelial cells are larger in size compared to central epithelial cells. Interestingly, in a study by Barr and Testa ${ }^{86}$ it was found that after eight hours of rigid gas permeable contact lens wear in subjects with 3- and 9-o'clock staining, the peripheral epithelial cell size significantly decreased. The peripheral mean cell area was found to be approximately half in size after lens wear compared to before lens wear. It was also found to be half the size compared to the epithelial cells of control subjects not wearing contact lenses. As 3and 9-o'clock staining increased in severity, the mean cell area of cells in the peripheral cornea decreased, but after one night of ceasing lens wear no statistically significant difference in cell size was found. It was hypothesized that the larger, more mature surface cells are desquamated prematurely due to either mechanical 
trauma by the contact lens or from desiccation of the corneal surface, while it appears that this can be overcome by one night of cessation of lens wear.

Looking at wound healing processes of the cornea: minor stages of corneal damage are believed to recover within hours and certainly overnight, ${ }^{40}$ while more severe cases of corneal damage can take up to 4 or 5 days to heal. The pathophysiology of wound repair in the more severe cases of corneal staining differs from that in the earlier stages. In minor cases of staining, epithelial basal cells at the corneal wound margin move into and cover the wound area within six hours after injury to seal off the damaged area. In larger abrasions, cell migration and adhesion takes place 2436 hours after the abrasion, followed by cell proliferation (which lasts 36 hours to weeks). ${ }^{87}$ Apart from wound size, the epithelial wound healing is also dependent on location. Central wounds tend to recover more slowly than peripheral ones. The rate of corneal wound healing is also dependent on the presence or absence of an epithelial basement membrane. When this membrane is present, re-epithelialization is faster, typically 2-3 days, while when absent the same process can take up to $5-7$ days. ${ }^{88}$

\section{Observation}

Damage to the corneal epithelium is viewed by clinicians through the use of Fluorescein $(\mathrm{NaFl})$ dyes. Fluorescein has been used for clinical diagnosis and research in the field of eye care since $1882 .{ }^{89-91}$ Fluorescein can be easily instilled into the tear film of the eye, and because the dye is highly water soluble it is in a very short time absorbed by cells that are physically in place but have permeable walls due to mechanical or physiological stress. Because fluorescein absorbs the blue and violet parts of the spectrum and emits fluorescent light of longer wavelength, it is ideal for use in clinical practice especially when viewed with cobalt blue light. However, the type and the concentration of fluorescein used, the fluid it is diluted with and the exact excitation wavelength can all influence the observed clinical appearance. ${ }^{92}$ A yellow barrier filter $(490 \mathrm{~nm})$ is used for excitation. The emitted light, in the visible spectrum of around $530 \mathrm{~nm}$ wavelength, can be better evaluated by the clinician. To enhance the visibility of the fluorescence, a barrier filter such as the Wratten \#12 provides greater contrast of the stained areas (Figure 3).

Practitioners have the choice of using either a $1 \%$ or a $2 \%$ fluorescein solution or to make use of disposable fluorescein strips. The latter option is preferred by most contact lens practitioners for hygienic reasons, but the concentration of the fluid applied to the eye with the strips depends heavily on the type of solution that is used to wet the strip and the amount of liquid that is present on the strip after wet- 
ting. It is advised to wet the strip with a sterile saline solution $(\mathrm{NaCl} 0.9 \%)$ and tap off any excess fluid before placing it on the ocular surface.

Soft contact lens materials will absorb the standard fluorescein dye that has a molecular weight of 376 . Therefore, soft lenses should always be removed before using fluorescein to evaluate the integrity of the ocular surface. As an alternative, fluorescein with a high molecular weight of 710 is also available that can be used with the soft lens on the eye. But the low molecular weight fluorescein is superior in showing details in epithelial damage compared to the high molecular weight fluorescein. ${ }^{5}$

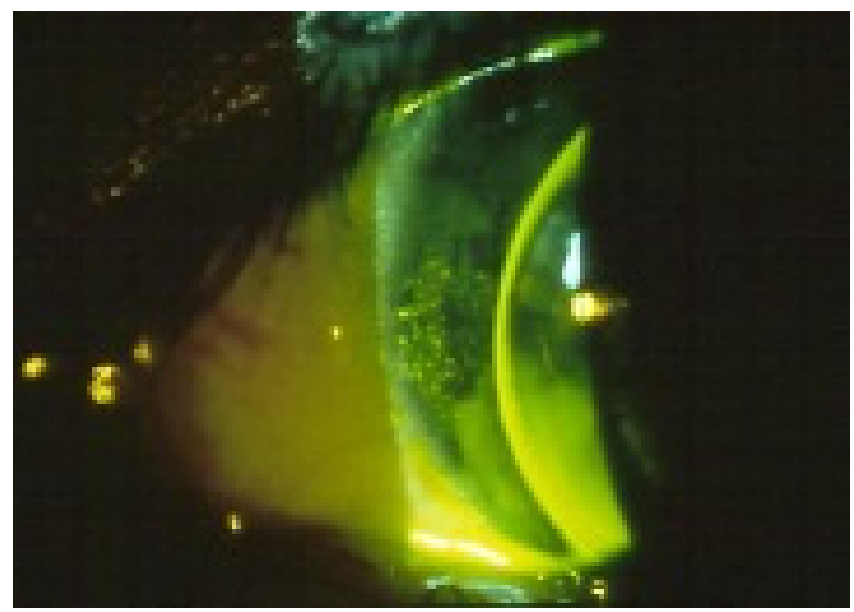

FIGURE 3. Observation of corneal staining in the 9-o'clock limbal area of the corneal surface using fluorescein.
Wilson et al. ${ }^{93}$ demonstrated that fluorescein primarily reveals cells that have taken up fluorescein optimally, which typically are degenerated or devitalized cells. Furthermore, to be clinically perceptible, a small group of maybe 5 to 10 cells needs to be dislodged together. Gaps in the surface which are left by single desquamating cells during the natural epithelial cell turnover would be too minute to be observed during routine slitlamp examination of the cornea. ${ }^{94}$

Clinicians should differentiate true corneal staining, caused by damage to the corneal epithelium, from other forms of fluorescein staining before labelling the staining. In contact lens wear, especially in rigid gas permeable lens wear and orthokeratology, the corneal epithelium can be indented by gas bubbles underneath the lens. Areas of fluorescein pooling may occur in the indented areas, but this does not represent true corneal staining (defined as damaged or devitalized cells). After a few blinks the pooling of staining disappears, while damaged epithelial cells may stain for minutes. ${ }^{95}$ 


\section{Grading}

Grading scales for ocular conditions can be of service for the evaluation of eye conditions and may be helpful in following the progression. With respect to epithelial corneal staining, one question that arises is: Is the extent or the depth of the staining more important clinically, or are both equally important? The Efron grading scale for contact lens complications ${ }^{96}$ grades corneal staining as a whole (combining depth and extent). The Vistakon grading scale takes both depth and type of staining into account, ${ }^{97}$ whereas the CCLRU grading scale ${ }^{98}$ differentiates depth, extent and type of staining (Table 2). Although the latter may be a more accurate method to describe the severity of the condition, it also is more time consuming and may therefore in the end be less effective in clinical settings than would grading epithelial staining as one entity.

TABLE 2. CCLRU descriptive grading scale for corneal staining ${ }^{98}$

\begin{tabular}{lll}
\hline Extent (surface area) & Depth & Type \\
\hline $1: 1-15 \%$ & 1 Superficial epithelium & 1 Micropunctate \\
2: $16-30 \%$ & 2 Deep epithelium, delayed stromal glow & 2 Macropunctate \\
3: $31-45 \%$ & 3 Immediate localized stromal glow & 3 Coalescent macropunctate \\
4: $>45 \%$ & 4 Immediate diffuse stromal glow & 4 Patch \\
\hline
\end{tabular}

It is common practice to divide the cornea into five segments when allocating the position of the staining on the cornea. The central portion is generally designated as area 1, the superior and inferior area as 4 and 5, while 2 and 3 represent the nasal and temporal areas respectively. In 3- and 9-o'clock staining, corneal staining is limited to the lateral portions of area 2 and 3 of the cornea (Figure 4).

For 3- and 9-o'clock corneal staining, several specific grading scales have been designed, all descriptive in nature and all combining type, extent and depth of the staining to assess the condition. ${ }^{86,99-104}$

Image grading scales could provide higher levels of accuracy as opposed to descriptive scales ${ }^{26}$ but no image grading scale is currently available for 3- and 9-o'clock corneal staining. In part 3.2 of this thesis, the development and testing of a new image grading scale specifically designed for 3-and 9-o'clock staining is outlined. 


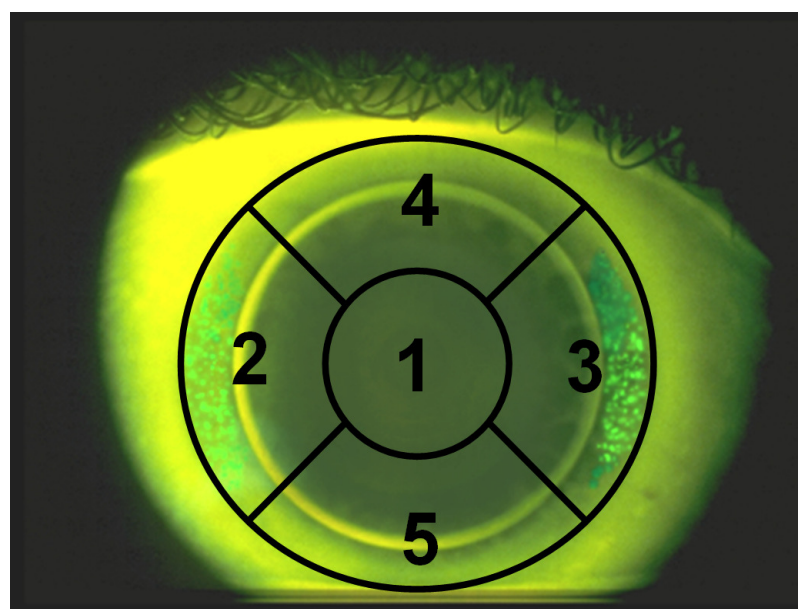

FIGURE 4. Location of corneal epithelial cell damage in 3- and 9-o'clock staining, limited to the lateral portions of area 2 and 3 of the cornea.

\section{Interpretation}

One of the key tasks for contact lens clinicians in daily practice is to evaluate the presence and amount of corneal staining, e.g. to assess the physiological status of the corneal epithelium. ${ }^{105}$ The prevalence of corneal staining in the contact lens wearing population is thought to be as high as $60 \%{ }^{65}$ Often, the presented staining is of a low level and generally clinically insignificant. ${ }^{106}$ To put things in perspective, corneal staining is frequently observed in non-lens wearers as well.65,90 Hamano et al. ${ }^{107}$ reported the prevalence of clinically significant amounts of staining (defined as grade 2 or higher) to be $0.9 \%$ in soft lens wearers and $0.5 \%$ in rigid gas permeable lens wearers.

Recently, corneal staining and its clinical relevance have gotten a lot of attention. Superficial epithelial damage caused by contact lens storage solutions has been linked to infectious and inflammatory events by some, ${ }^{90,108,109}$ but others have disputed this relationship stating that the mechanism behind corneal infectious disease is complex and multifactorial and cannot simply be attributed to corneal staining. ${ }^{105,110}$ It appears that superficial punctate patterns of fluorescein staining (as seen in solution toxicity) should be viewed differently from non-superficial coalesced injury-related staining. ${ }^{105}$

With regard to 3-and 9-o'clock staining: it has been reported that $80 \%$ of rigid gas permeable wearers show some degree of staining, ${ }^{111}$ but it is estimated to be of clinical significance in only $10-15 \% .{ }^{111,112}$ But the state of 'clinically significant' is undefined. As with any other condition, 3-and 9-o'clock staining corneal staining 
above stage two should be considered as highly clinically significant. Typically, grade 2 on $0-4$ clinical grading scales is accepted as a critical clinical cut-off point for the management of contact lens related conditions, ${ }^{96,98}$ and clinical action usually is required. Apart from a possible gateway for ocular pathogens to the stroma, there is an increased risk of corneal scarring if the anterior limiting membrane is affected. ${ }^{88,113}$ In severe cases, 3- and 9-o'clock staining may cause localized thinning of the cornea in the limbal areas and a vascularized limbal keratitis may develop. This includes corneal vascularization, sub-epithelial infiltration, scarring and hyperplasia. This is seen more in EW of rigid gas permeable lenses, but can present itself in DW as well. ${ }^{114}$

The clinical consequence of lower levels of 3- and 9-o'clock corneal staining is hard to define. A clear association between dry eye, punctate corneal staining and tear film integrity has been reported, ${ }^{115-118}$ but no studies directly analyzing the possible risks involved in 3- and 9-o'clock staining are available. As a general rule in clinical practice, it is accepted that 'brighter is worse' and 'more is bad' when it comes to corneal staining. In many studies, the presence of corneal staining is considered a complication of lens wear, and generally it is believed that it is better to avoid 3and 9-o'clock staining at any time if possible, since it represents an alteration in corneal integrity caused by contact lens wear. ${ }^{116}$

\section{The Tear Film}

The most outer surface of the epithelium is covered by numerous tiny microvilli, and one of the main proposed functions of these is to stabilize the tear film on the ocular surface. The average thickness of the tear film varies from approximately 9 $\mu \mathrm{m}$ immediately after an eyeblink to around $4 \mu \mathrm{m}$ just before the next eyeblink. ${ }^{119}$ An average contact lens exceeds the thickness of the tear film, which can cause an increase in tear film evaporation. The simple presence of a contact lens itself may be the challenging factor causing dry eye symptoms in an otherwise perfectly normal functioning eye. ${ }^{119}$

The normal tear film is classically described as a three laminar structure comprising a superficial lipid layer, an intermediate aqueous phase and an underlying mucous layer. ${ }^{120,121}$ The central aqueous phase of the tear film is approximately 6.5 to 7.5 $\mu \mathrm{m}$ thick, ${ }^{122}$ which is the bulk of the total tear film. The aqueous phase contains dissolved ions and proteins and is thought to be an important provider of nutrition to the cornea. This aqueous phase is covered by a layer of lipids (the lipid layer) which is only around $0.1 \mu \mathrm{m}$ in thickness. The main function of the lipid layer is 
to prevent evaporation of the underlying water from the aqueous phase. ${ }^{119}$ More recently, investigators have shown that the tear film is more complex than originally believed, containing additional layers and interfaces. Especially the interface between the aqueous phase and the corneal epithelium deserves a more detailed description. Mucins, which form the third and most deeply located layer in the classic tear film composition, play a vital role in that interface.

The corneal microvilli overlaying the epithelial cells are covered with mucins, and it is the combination of microvilli and mucins that is believed to be capable of attracting the tear layer to the otherwise hydrophobic cornea. This ensures a clear optical surface that serves as the main refractive medium of the human eye. ${ }^{94}$ Apart from maintenance of tear film on the ocular surface, another function of mucins is provision of a pathogen barrier on the wet ocular surface, as well as lubrication. When areas of surface damage occur, mucins seem to play an essential role in overcoming the temporary hydrophobicity of the cornea. ${ }^{123}$

To date, 16 human mucin (MUC) genes have been identified. The primary secretory mucin is the large gel-forming MUC5AC that is produced by conjunctival goblet cells and the crypts of Henle in the fornices of the anterior eye. ${ }^{119}$ However, the stratified epithelium of the cornea and conjunctiva also expresses mucins; at least three transmembrane mucins have been found (MUC1, MUC4 and MUC16). They are an extension of the epithelial cell surface and form the glycocalyx of apical microvilli. The transmembrane mucins and the gel-forming mucins in the tear film attach to each other and work together to achieve tear spreading after lid opening. After each eyeblink, the gel-forming mucins spread over the transmembrane produced glycocalyx layer. This facilitates the spread and attachment of the aqueous layer to the corneal epithelium. ${ }^{119}$

Liotet et al. ${ }^{124}$ in 1987 described a hypothesis on tear film stability, stating that epithelial cell integrity is essential for the stability of the tear film. Theoretically, this could play a role in the etiology of 3-and 9-o'clock staining. More on possible mechanisms behind the condition can be found in part 3.1 of this thesis.

\section{Tear Volume}

The total volume of tears present on the ocular surface within the palpebral aperture has been estimated to be between $7 \mu \mathrm{l}$ and $10 \mu \mathrm{l} .{ }^{125}$ Any fluid volume above $10 \mu \mathrm{l}$ is thought to be flushed out of the normal palpebral fissure, although this will vary with the size of the palpebral fissure. Of the total volume of tear fluid present within the palpebral aperture, about $70-90 \%$ is thought to be consumed by the tear menisci lining the upper and the lower eyelid margins. ${ }^{119,122,126}$ When a rigid 
gas permeable contact lens is placed on the ocular surface, another tear meniscus forms that surrounds the entire edge of the lens. According to Holly ${ }^{127}$ and others $^{128,129}$ this could be one of the underlying causes of 3- and 9-o'clock staining: capillary attraction of tears to the tear meniscus adjacent to the lens edge could withdraw substantial amounts of fluids. A more detailed description on the tear volume or tear meniscus theory can be found in part 3.1 of this thesis.

Measuring tear volume in clinical practice is not an easy task. The classical method to get an indication of the available tear volume on the ocular surface was introduced in 1903 by Schirmer, ${ }^{130}$ but it was not until 1941 when Whatman \#41 filter paper was used to standardize the procedure that the test became widely available. ${ }^{131}$ Using this technique, small strips of the filter paper are placed near the outer cantus on the lower eyelid, and the amount of wetting of the filter paper by tears in a particular time period is registered. Different versions of the test have been developed, with and without the use of an anesthetic. The latter was suggested to avoid reflex tearing, but some reflex tearing seems to occur even despite instillation of the anesthetic. ${ }^{131}$ Based on these and other considerations, the National Eye Institute in the USA recommended that the Schirmer test without an anesthetic is a better measure of the capacity of the lacrimal glands to produce tears than with the use of an anesthetic. ${ }^{131,132}$ Despite the risk of reflex tearing and the discomfort for the subject while performing the test, Schirmer tests provide a probable diagnosis of an aqueous deficient eye if the outcome after 5 minutes is less than $5 \mathrm{~mm}$ of wetting of the filter paper. If the value is below $3 \mathrm{~mm}$ within that same timeframe, the chance of an aqueous-deficient eye is considered likely. Schirmer test values of between $6 \mathrm{~mm}$ and $10 \mathrm{~mm}$ can be considered marginal aqueous-deficient eyes, but this outcome cannot be considered conclusive since there is no assurance that reflex tearing due to the irritation of the paper strip was absent.

The phenol red test is a modified Schirmer measurement in which the filter strip is replaced with a fine cotton thread stained with phenol red. ${ }^{130,131}$ A change of coloration of the thread from orange to red reveals the length of thread that is wetted by the alkali tears. The thread is inserted into the lower tear meniscus for 15 seconds, and a tear production deficiency is suspected when less than $10 \mathrm{~mm}$ of the thread is wetted within that timeframe. In view of its less invasive nature than the Schirmer test, the chances of having reflex tearing seem reduced. ${ }^{133}$

Measurement of the tear meniscus height (TMH) on the lower eyelid has been proposed by many as an alternative test in this regard. ${ }^{121,134-138}$ Observation and measurement of the TMH and the regularity of the tear meniscus has been sug- 
gested as a simple and fast alternative to get an indication of the available tear volume on the ocular surface, ${ }^{125}$ including during contact lens wear. ${ }^{139}$ A scanty appearance of the lower tear meniscus or local areas of discontinuity are believed to be signs of a tear film deficiency. A TMH of less than $0.3 \mathrm{~mm}$ has been considered as an indication of a dry eye, ${ }^{59,125,140}$ but measurement is difficult in clinical settings. TMH measurements have been found to correlate well with results of the phenol red test and tear stability tests. ${ }^{137,141}$ However, although currently available tear volume tests such as the Schirmer test, phenol red test and TMH measurement may be able to reveal pathological dry eye situations, many investigators have pointed out that there is a high variability in outcome and that it is unclear whether these tests are able to detect minor aqueous deficiencies. Especially small changes to the tear volume on the anterior eye that may result from contact lens wear may be difficult to determine. In part 5.1, TMH in relation to rigid gas permeable lens wear and 3- and 9-o'clock staining is investigated and discussed.

\section{Tear Film Stability}

The more stable a tear film is, the better the protection of the epithelium against the environment seems to be. ${ }^{130}$ The lipid layer of the tear film is important to retard evaporation of the underlying aqueous layer in order to retain stability of the tear film. A four-fold increase in tear evaporation is observed when the human lipid layer is absent. ${ }^{142}$ The lipid layer is comprised of polar and non-polar lipids. Disturbance of the lipid layer, as happens in contact lens wear, causes migration of lipids towards the mucous layer on the corneal surface, and contamination of this mucous layer may result in small hydrophobic areas. This occurance has generally been accepted as the mechanism responsible for breakup of the tear film. When a thinner aqueous phase is present, migration of lipids and contamination of the mucous layer happens faster. The time it takes for a tear film to break up can be used as a general indicator of the stability of the ocular tear film. The measured time before the tear film breaks up is generally referred to as the tear break up time (BUT). The normal tear film in humans does not remain stable for long after an eyeblink; ${ }^{127}$ when eyeblinking is prevented the tear film ruptures usually within 15 40 seconds. ${ }^{143}$ A tear film with a BUT of less than 10 seconds is generally considered unstable from a clinical perspective. The quality of the BUT test has been questioned frequently because of its high variance, mainly caused by the need to instill fluids (fluorescein). But if performed correctly, this test can be repeatable and reliable especially when the amount of fluorescein added to the tear film is limited and controlled. ${ }^{144}$

To overcome the variation of the BUT values, non-invasive techniques have been proposed to assess tear film stability. By projecting an image on the pre-ocular tear 
film and observing changes in the specular image, the non-invasive break up time (NIBUT) can be measured. ${ }^{133}$ What is actually measured when using this technique is the tear thinning time because the tear film not necessarily breaks up completely before changes in the projected image are visible. NIBUT values are significantly higher than BUT values: the normal NIBUT range is typically between 40 60 seconds. The NIBUT test is in theory an ideal method for measuring tear stability, but it is not in routine clinical use because if done properly it requires special instrumentation that is not widely available in contact lens practices. ${ }^{131}$ Pflugfelder et al. ${ }^{145}$ argued that the NIBUT test does not replace the BUT test but rather evaluates a different phenomenon than the NIBUT test, namely the integrity of the precorneal mucin layer rather than the overall stability of the tear film.

While wearing contact lenses, BUT and NIBUT values are significantly lower than the BUT and NIBUT values in non-lens wearing eyes. ${ }^{139}$ Usually it takes only a few seconds for the pre-lens tear film to break up, or the BUT and NIBUT are entirely absent (meaning the tear film breaks up immediately after an eyeblink). ${ }^{146}$

Corneal desiccation staining is believed to be part of a mechanism that involves the tear film stability and eyeblink frequency. At some time after an eyeblink the tear film starts to break up as described, and the ocular surface may be exposed to the environment from that point on and discomfort can occur as a result. At that point in time an eyeblink must take place in order to protect the ocular surface, otherwise corneal staining may occur. Consequently, there is a relationship between the BUT of the tear film and the time between eyeblinks (the inter eyeblink interval, IEBI). The BUT divided by the IEBI provides the ocular protection index (OPI) ${ }^{147}$ An OPI larger than one is considered sufficient in creating a protected ocular surface. An OPI of less than one results in an unprotected ocular surface, which can potentially lead to corneal staining.

\section{Eyeblinking}

In the past 75 years, many investigators have reported on eyeblink rates. Since the first reports on eyeblink frequency, large variations in spontaneous eyeblink rate (SEBR) have been found in individuals. SEBR depends for instance on the subjects' direction of gaze during the test. ${ }^{148,149}$ The SEBR value ( \pm standard deviation) for reading tasks was found to be $7.9 \pm 3.3$ eyeblinks/minute on average based on 16 literature reports analyzed by Doughty. ${ }^{150}$ The average SEBR in primary eye gaze (based on 22 studies) was found to be $14.5 \pm 3.3$ eyeblinks/minute, and the average SEBR during conversation was $21.5 \pm 5.6$ eyeblinks/minute based on 14 sets of reports. Variations in eyeblink rates can also occur based on the subjects' 
task: watching a video tape, ${ }^{151}$ conversing ${ }^{152}$ and looking at a silent object ${ }^{153}$ can result in different eyeblink rates, even with the direction of gaze being the same. Most studies on eyeblink rate observe it in primary eye gaze while the participant is looking at a silent object. ${ }^{150,153-157}$

The definition of an eyeblink is a discussion in itself. Usually an eyeblink is described in the literature as a 'discernible movement of the upper eyelid causing an obvious reduction in the size of the palpebral fissure, ${ }^{154,158}$ although it often remains unclear how large that discernible movement exactly is. Many studies do not specify what is considered an eyeblink action and what is not. ${ }^{159-163}$ Categorization of eyeblinks based on the amount of reduction in aperture height was suggested by Abelson and Holly ${ }^{154}$ in 1977. They subdivided unforced eyeblinks into either complete eyeblinks, incomplete eyeblinks or twitch eyeblinks. They describe complete eyeblinks as an eyeblink action in which the upper eyelid covers more than two-thirds of the cornea. ${ }^{154}$ Incomplete eyeblinks are described as 'the descending upper eyelid covering less than two-thirds of the cornea' and a twitch eyeblink is defined broadly as 'an almost undetectable small movement of the upper eyelid'. Some studies use a 'forceful eyeblink' in addition to these as a separate category. This is described as a voluntary, forced eyeblink in which the lower eyelid is raised, producing a near squint, ${ }^{151-156,163,164}$ but this type of eyeblink appears to be very rare. ${ }^{151,152,154-156}$

Differences in eyeblink frequency and eyeblink amplitude in contact lens wear have been cited in the literature, ${ }^{156,159,160,165}$ although the number of studies on this subject is limited with regard to rigid gas permeable lens wear. Based on theoretical considerations and clinical observation, alteration in eyeblink behavior during rigid gas permeable lens wear can be a possible risk factor for the development of 3-and 9-o'clock staining. ${ }^{59,166,167}$ The assumption is made that discomfort inhibits eyeblinking, which stimulates tear evaporation, and this in turn can stimulate the development of 3- and 9-o'clock staining, ${ }^{168,80}$ but no publications studying this potential relationship are available. Eyeblink behavior in rigid gas permeable contact lens wear will be covered and studied in detail in part 5.2 of this thesis. 


\section{Aims and Outline of this Thesis}

The primary aim of this thesis is to better understand the mechanism behind corneal desiccation in rigid gas permeable lens wear (3- and 9-o'clock staining) and to provide the contact lens practitioner with clinical tools to better manage the condition.

A general introduction into the subject and the main challenges regarding rigid gas permeable contact lens wear are described in chapter 1 of this thesis. Chapter 2 focuses on whether comfort of lens wear can be improved by optimizing the lens fits and evaluates whether better methods are available today than the traditional fitting techniques to achieve this. In chapter 3 , part 3.1 provides an overview of the condition; while in part 3.2 a specially designed image grading scale for 3- and 9o'clock corneal staining is introduced. Chapter 4 of this thesis presents the results of clinical studies evaluating 3- and 9-o'clock staining in existing rigid gas permeable lens wearers. The relationship between the signs and the symptoms accompanying 3- and 9-o'clock staining (bulbar hyperemia, conjunctival staining and comfort of lens wear) is investigated in part 4.1, while part 4.2 evaluates contact lens related variables that could potentially contribute to the development of the condition. In chapter 5, possible mechanisms behind the development of 3- and 9o'clock staining are analysed. Part 5.1 describes a study comparing tear meniscus height (as a representative of tear volume) in rigid gas permeable lens wearers (with and without 3- and 9-o'clock corneal staining) with a control group of non-lens wearers. Part 5.2 looks at the quantity and quality of eyeblinks in rigid gas permeable lens wear. It investigates the possible difference in frequency and in completeness of eyeblinks in non-lens wearers and in rigid gas permeable lens wearers (with and without 3-and 9-o'clock staining). Finally, in chapter 6 the results of the studies are summarized and placed in a broader perspective. 


\section{References}

1. Vinci da L. Codex of the Eye, Manuscript D. 1508.

2. Enoch JM. Descartes' contact lens. Am J Optom Arch Am Acad Optom 1956;33:77-85.

3. Bowden T, Gasson A. Overseas Contact Lens Pioneers. In: BCLA Pioneers' Conference; 2006; p. 2-4.

4. Fick A. Eine Contactbrille. Archive für Augenheilkunde 1888:279-89.

5. Panas P. Presentation of instruments and apparatus: A discussion of Kalt's use of shells of glass against keratoconus. Bull Acad Med 1888;3:400-1.

6. Rohr von M, Boegehold H. Das Brillenglas als Optisches Instrument. In. Berlin: Springer, 1934:17.

7. Koetting R. Contact Lenses. New York: Gower Medical Publishing, 1991.

8. Burger R. Effect of contact lens motion on the oxygen tension distribution under the lens. Am J Optom Physiol Opt 1974;57:441.

9. Holden B, Mertz G. Critical oxygen levels to avoid corneal edema for both daily and extended wear contact lenses. Invest Ophthalmol Vis Sci 1984;25:1161-7.

10. Finnemore VM, Korb JE. Corneal edema with polymethylmethacrylate versus gas-permeable rigid polymer contact lenses of identical design. J Am Optom Assoc 1980;51:271-4.

11. Holden B, Mertz G. Critical oxygen levels to avoid corneal edema for daily and extended wear contact lenses. Invest Ophthalmol Vis Sci 1984:1161-7.

12. Hirst E. Fitting a true aspherical contact lens. Contactologia 1974;1:15-9.

13. Van der Worp E, De Brabander J, Lubberman B, Marin G, Hendrikse F. Optimising RGP lens fitting in normal eyes using 3D topographic data. Cont Lens Anterior Eye 2002;25:95-9.

14. Polse K. Tear Flow under hydrogel contact lenses. Invest Ophthalmol Vis Sci 1979;18:409-13.

15. Lai $Y$, Friends G. Surface wettability enhancement of silicone hydrogel lenses by processing with polar plastic molds. J Biomed Mater Res 1997;35:349-56.

16. De Brabander J, Kok JH, Nuijts RM, Wenniger-Prick LJ. A practical approach to and longterm results of fitting silicone contact lenses in aphakic children after congenital cataract. CLAO J 2002;28:31-5.

17. Efron N. Are silicone hydrogel lenses safer? Cont Lens Anterior Eye 2005;28:153-5.

18. Amos C, Grant T. Optimizing Oxygen Without Compromising Performance. Contact Lens Spectrum 1999.

19. Morgan P, Efron N, Woods $C$ et al. International contact lens prescribing 2007. Contact Lens Spectrum 2008;23(1):36-41.

20. Ciba Vision. Global Contact Lens Penetration Study; 2007 (June 2006-June 2007).

21. NAC-report. Kwantitatief onderzoek lenzenmarkt (Nederland) 2007 Hogeschool Drenthe 2008:1-57.

22. Morgan P, Efron N, Woods C et al. International contact lens prescribing 2006. Contact Lens Spectrum 2007;22(1):34-8.

23. Dorronsoro C, Barbero S, Llorente L, Marcos S. On-eye measurement of optical performance of rigid gas permeable contact lenses based on ocular and corneal aberrometry. Optom Vis Sci 2003;80:115-25.

24. Hong X, Himebaugh N, Thibos LN. On-eye evaluation of optical performance of rigid and soft contact lenses. Optom Vis Sci 2001;78:872-80.

25. Lu F, Mao X, Qu J, Xu D, He JC. Monochromatic wavefront aberrations in the human eye with contact lenses. Optom Vis Sci 2003;80:135-41.

26. Cano D, Barbero S, Marcos S. Comparison of real and computer-simulated outcomes of LASIK refractive surgery. J Opt Soc Am A Opt Image Sci Vis 2004;21:926-36. 
27. Marcos S, Cano D, Barbero S. Increase in corneal asphericity after standard laser in situ keratomileusis for myopia is not inherent to the Munnerlyn algorithm. J Refract Surg 2003;19:S592-6.

28. Sorbara LG, Woods CA. Correction of Presbyopia with GP Contact Lenses. Waterloo: The Centre for Contact Lens Research, Waterloo, Canada, 2007.

29. Bennett ES. Contact lens correction of presbyopia. Clin Exp Optom 2008;91:265-78.

30. Cheng KH, Leung SL, Hoekman HW et al. Incidence of contact-lens-associated microbial keratitis and its related morbidity. Lancet 1999;354:181-5.

31. Chalupa E, Swarbrick HA, Holden BA, Sjostrand J. Severe corneal infections associated with contact lens wear. Ophthalmology 1987;94:17-22.

32. Poggio EC, Glynn RJ, Schein OD et al. The incidence of ulcerative keratitis among users of daily-wear and extended-wear soft contact lenses. N Engl J Med 1989;321:779-83.

33. Nilsson SE. [Ulcerative keratitis as a complication of contact lenses]. Lakartidningen 1990;87:2245-7.

34. Ladage PM, Yamamoto K, Li L et al. Corneal epithelial homeostasis following daily and overnight contact lens wear. Cont Lens Anterior Eye 2002;25:11-21.

35. Ren DH, Petroll WM, Jester JV, Ho-Fan J, Cavanagh HD. The relationship between contact lens oxygen permeability and binding of Pseudomonas aeruginosa to human corneal epithelial cells after overnight and extended wear. CLAO J 1999;25:80-100.

36. Efron N, Morgan PB, Hill EA, Raynor MK, Tullo AB. Incidence and morbidity of hospitalpresenting corneal infiltrative events associated with contact lens wear. Clin Exp Optom 2005;88:232-9.

37. Efron N, Morgan PB, Hill EA, Raynor MK, Tullo AB. The size, location, and clinical severity of corneal infiltrative events associated with contact lens wear. Optom Vis Sci 2005;82:519-27.

38. Morgan PB, Efron N, Hill EA, Raynor MK, Whiting MA, Tullo AB. Incidence of keratitis of varying severity among contact lens wearers. Br J Ophthalmol 2005;89:430-6.

39. Phillips A. Rigid gas permeable and hard corneal lens fitting. In: Phillips A, Stone J, ed. Contact Lenses. London: Butterworth, 1989:333-81.

40. Efron N. Contact Lens Complications. Oxford: Butterworth-Heinemann, 1999.

41. Yamane SJ. Are hard lenses superior to soft? The advantages of soft lenses. Cornea 1990;9 Suppl 1:S12-4; discussion S5.

42. Fonn D, Gauthier CA, Pritchard N. Patient preferences and comparative ocular responses to rigid and soft contact lenses. Optom Vis Sci 1995;72:857-63.

43. Johnson M, Chen A, Epstein DL, Kamm RD. The pressure and volume dependence of the rate of wash-out in the bovine eye. Curr Eye Res 1991;10:373-5.

44. Vajdic C, Holden BA, Sweeney DF, Cornish RM. The frequency of ocular symptoms during spectacle and daily soft and rigid contact lens wear. Optom Vis Sci 1999;76:705-11.

45. Bennett ES, Smythe J, Henry VA et al. Effect of topical anesthetic use on initial patient satisfaction and overall success with rigid gas permeable contact lenses. Optom Vis Sci 1998;75:800-5.

46. Kok J. New developments in the field of contact lenses. Amsterdam: University of Amsterdam; 1991.

47. Van der Worp E, De Brabander J, Jongsma F. Corneal topography. In: Bennett E, Henry V, ed. Clinical Manual of Contact Lenses, 3rd ed. Philadelphia: Lippincott Williams \& Wilkins, 2008:48-78.

48. Von Helmholz H. Graefes's Archiv Ophthalmol;1854:3.

49. Javal E, Schiötz I. Un opthalmomètre practique. Ann Oculis 1881;84:5.

50. Placido A. Novo instrumento de exploracao da cornea. Periodico d'Ophthalmologica Practico, Lisbon 1880;5:27-30. 
51. Naroo SA, Cervino A. Corneal Topography and its role in refractive surgery. In: Naroo SA, ed. Refractive Surgery: A Guide to Assessment and Management. London: ButterworthHeinemann, 2004:9-17.

52. Sicam VA, Van der Heijde RG. Topographer reconstruction of the nonrotation-symmetric anterior corneal surface features. Optom Vis Sci 2006;83:910-8.

53. Iskander DR, Collins MJ. Applications of high-speed videokeratoscopy. Clin Exp Optom 2005;88:223-31.

54. Swartz T, Marten L, Wang M. Measuring the cornea: the latest developments in corneal topography. Curr Opin Ophthalmol 2007;18:325-33.

55. Jongsma FH, de Brabander J, Hendrikse F, Stultiens BA. Development of a wide field height eye topographer: validation on models of the anterior eye surface. Optom Vis Sci 1998;75:6977.

56. Vos F, Heijde van der G, Spoelder H, Stokkum van I, Groen F. A new PRBA-based instrument to measure the shape of the cornea. IEEE Trans Instrum Meas 1997:794-7.

57. Dunne MC, Davies LN, Wolffsohn JS. Accuracy of cornea and lens biometry using anterior segment optical coherence tomography. J Biomed Opt 2007;12:064023.

58. Guillon J-P, Young G. Subtle signs of sicca. Optician 1999;218:18-20.

59. Lowther GE. Dryness, Tears, and Contact Lens Wear: Clinical Practice in Contact Lenses. Boston: Butterworth-Heinemann, 1997:84-90.

60. Brennan N, Efron N. Symptomatology of HEMA contact lens wear. Optom Vis Sci 1989;66:834-8.

61. Tomlinson A. Contact lens-induced dry eye. In: Tomlinson A, ed. Complications in Contact Lens Wear. St. Louis: Mosby, 1992:195-218.

62. Fonn D. Targeting contact lens induced dryness and discomfort: what properties will make lenses more comfortable. Optom Vis Sci 2007;84:279-85.

63. Pascucci $S$, et al. An analysis of age related morphologic changes of human meibomian glands. Invest Ophthalmol Vis Sci 1988;29:213.

64. Orsborn G, Robby M. Hydrogel lenses and dry eye symptoms. Journal of the British Contact Lens Association 1989;6:37.

65. Guillon JP, Guillon M, Malgouyres S. Corneal desiccation staining with hydrogel lenses: tear film and contact lens factors. Ophthalmic Physiol Opt 1990;10:343-50.

66. Zadnik K, Mutti D. Inferior arcuate staining with thin high water content contact lenses. Int Contact Lens Clin 1985;12:110.

67. Holden B, Sweeney D, Seger R. Epithelial erosions caused by thin high water contact lenses. Clinical and Experimental Optometry 1986;69:103-7.

68. Little S, Bruce A. Role of post-lens tear film in the mechanism of inferior arcuate staining with ultrathin hydrogel lenses. CLAO J 1995;21:175-81.

69. Efron N, Brennan N, O'Brien K, PJ M. Surface hydration of hydrogel contact lenses. Clinical and Experimental Optometry 1986;69:219-22.

70. Benedotto D, Laibson P, TE C. In vivo observation of the tear dynamics using Fluorphotometry. Arch. Ophthalmology 1984;102:410-2.

71. Efron N, Young G. Dehydration of hydrogen contact lenses in vitro and in vivo. Ophthalmic Physiol Opt 1988;8:253-6.

72. Brennan NA, Lowe R, Efron N, Ungerer JL, Carney LG. Dehydration of hydrogel lenses during overnight wear. Am J Optom Physiol Opt 1987;64:534-9.

73. Zantos S. Studies on corneal staining with thin hydrogel contact lenses. Journal of the British Contact Lens association 1986;9:61-4.

74. Orsborn G, Zantos S. Corneal desiccation staining with thin high water content contact lenses. CLAO J 1988;14:81-5. 
75. Hall B, Jones S, Young G, Coleman S. The on-eye dehydration of proclear compatibles lenses. CLAO J 1999;25:233-7.

76. Fonn D, Situ P, Simpson T. Hydrogel lens dehydration and subjective comfort and dryness ratings in symptomatic and asymptomatic contact lens wearers. Optom Vis Sci 1999;76:700-4.

77. Young G, Bowers R, Hall B, Port M. Six month clinical evaluation of a biomimetic hydrogel contact lens. CLAO J 1997;23:226-36.

78. Young G, Bowers R, Hall B, Port M. Clinical comparison of Omafilcon A with four control materials. CLAO J 1997;23:249-58.

79. Lemp $\mathrm{M}$, al e. Omafilcon A (proclear) soft contact lenses in a dry eye population. CLAO J 1999;25:40-7.

80. Van der Worp E, De Brabander J, Swarbrick H, Nuijts R, Hendrikse F. Corneal desiccation in rigid contact lens wear: 3- and 9-o'clock staining. Optom Vis Sci 2003;80:280-90.

81. Steele E. Observations on the fitting of corneal contact lenses. Am J Optom Arch Am Acad Optom 1959;36:194-9.

82. Jones L, Jones D. Common Contact Lens Complications. Oxford: Butterworth-Heinemann, 2000:70-3.

83. Li HF, Petroll WM, Moller-Pedersen T, Maurer JK, Cavanagh HD, Jester JV. Epithelial and corneal thickness measurements by in vivo confocal microscopy through focusing (CMTF). Curr Eye Res 1997;16:214-21.

84. Efron N, Matalib A, Gomez I. Confocal microscopy of the edematous cornea following overnight lens wear. In: American Acadamy of Optometry; 1999 December 11; Seattle.

85. Hanna C, O'Brien J. Cell production and migration in the epithelial layer of the cornea. Arch Ophthalmol 1960;64:536-41.

86. Barr J, Testa L. Corneal epithelium 3 and 9 o'clock staining studied with the specular microscope. Int Contact Lens Clin 1994;21:105-11.

87. Steele C. Corneal wound healing: a review. Optometry Today 1999:28-32.

88. Doshi S. Corneal anatomy, physiology and response to wounding. In: Naroo SA, ed. Refractive Surgery: A Guide to Assessment and Management. London: Butterworth-Heinemann, 2004:1726.

89. Fromm, Groenouw. Ueber die diagnostische Verwendbarkeit der Fluoresceinfarbung bei Augenerkrankungen. Archive fuer Augenheilkunde 1891;22:247-57.

90. Norn MS. Micropunctate fluorescein vital staining of the cornea. Acta Ophthalmol (Copenh) 1970;48:108-18.

91. Pflueger. Zur Ernaehrung der Cornea. Klinisches Monatsblaetter Augenheilkunde 1882;20:6981.

92. De Brabander J, Jongsma F, Van Rooij, Hendrikse F. Tear Film Fluorescence. PhD thesis. Maastricht: University of Maastricht; 2002.

93. Wilson G, Ren H, Laurent J. Corneal epithelial fluorescein staining. J Am Optom Assoc 1995;66:435-41.

94. Bergmanson J. Light and electron microscopy. In: Efron N, ed. The Cornea. Oxford: Butterworth-Heinemann, 2001:136-78.

95. Weissman BA. Assessing and Treating Complications. Contact Lens Spectrum 2006;21(6):529.

96. Efron N. Grading scales for contact lens complications. Ophthalmic Physiol Opt 1998;18:1826.

97. Andersen JS, Davies IP, Kruse A, Løfstrom T, Ringmann LA. Handbook of Contact Lens Management. Jacksonville: Vistakon, 1996.

98. Terry RL, Schnider CM, Holden BA et al. CCLRU standards for success of daily and extended wear contact lenses. Optom Vis Sci 1993;70:234-43. 
99. Bennet E. The effect of varying axial edge lift on silicone/acrylate lens performance. Contact Lens Journal 1986;14:3-7.

100. Andrasko G. Peripheral corneal staining: incidence and time course. Contact Lens Spectrum 1990;5(7):59.

101. Schnider C, Terry R, Holden B. Effect of lens design on peripheral corneal desiccation. J Am Optom Assoc 1997;68:163-70.

102. Brannen R. Incidence of central edema and peripheral staining as a function of contact lens fitting philosophy. J Am Optom Assoc 1977;48:391-5.

103. Lebow K. Peripheral corneal staining. In: Silbert J, ed. Anterior Segment Complications of Contact Lens Wear. Edinburgh: Churchill Livingstone, 1994:59-90.

104. Henry VA, Bennett ES, Forrest JF. Clinical investigation of the Paraperm EW rigid gaspermeable contact lens. Am J Optom Physiol Opt 1987;64:313-20.

105. Ward KW. Superficial punctate fluorescein staining of the ocular surface. Optom Vis Sci 2008;85:8-16.

106. Efron N. Staining. In: Efron N, ed. Contact Lens Complications. Oxford: ButterworthHeinemann, 1999:75-81.

107. Hamano H, Kitano J, Mitsunaga S, Kojima S, Kissling GE. Adverse effects of contact lens wear in a large Japanese population. CLAO J 1985;11:141-7.

108. Li SL, Ladage PM, Yamamoto T, Petroll WM, Jester JV, Cavanagh HD. Effects of contact lens care solutions on surface exfoliation and bacterial binding to corneal epithelial cells. Eye Contact Lens 2003;29:27-30.

109. Cavanagh HD. Fusarium, contact lens solutions, and patient compliance: a tangled, critical web. Eye Contact Lens 2006;32:255.

110. Levy B. Superficial corneal "staining"-clinical observation and risk assessment. Eye Contact Lens 2007;33:165-6.

111. Solomon J. Causes and treatments of peripheral corneal desiccation. Contact Lens Forum 1986;11:30-6.

112. Ghormley N, Bennett E, Schnider C. Corneal desiccation-clinical management. Int Contact Lens Clin 1990;17:5.

113. Naroo SA. Refractive Surgery: A Guide to Assessment and Management. London: ButterworthHeinemann, 2004:17-26.

114. Grohe R, Lebow K. Vascularized limbal keratitis. Int Contact Lens Clin 1989;16:197-209.

115. Yokoi N, Takehisa Y, Kinoshita S. Correlation of tear lipid layer interference patterns with the diagnosis and severity of dry eye. Am J Ophthalmol 1996;122:818-24.

116. Nichols KK, Mitchell GL, Simon KM, Chivers DA, Edrington TB. Corneal staining in hydrogel lens wearers. Optom Vis Sci 2002;79:20-30.

117. Nichols KK, Nichols JJ, Lynn Mitchell G. The relation between tear film tests in patients with dry eye disease. Ophthalmic Physiol Opt 2003;23:553-60.

118. Bourcier T, Acosta MC, Borderie V et al. Decreased corneal sensitivity in patients with dry eye. Invest Ophthalmol Vis Sci 2005;46:2341-5.

119. Korb D, Craig J, Doughty M, Guillon J-P, Smith G, Tomlinson A. The Tear Film: structure, function and clinical examination. London: Butterworth-Heinemann, 2002:18-51.

120. Wolff $\mathrm{E}$. The muco-cutaneous junction of the lid margin and the distribution of the tear fluid. J Br Contact Lens Association 1946;66:291.

121. Holly FJ, Lemp MA. Tear physiology and dry eyes. Surv Ophthalmol 1977;22:69-87.

122. Mishima S, Gasset A, Klyce SD, Jr., Baum JL. Determination of tear volume and tear flow. Invest Ophthalmol 1966;5:264-76.

123. Tiffany JM. Composition and biophysical properties of the tear film: knowledge and uncertainty. Adv Exp Med Biol 1994;350:231-8. 
124. Liotet S, Van Bijsterveld OP, Kogbe O, Laroche L. A new hypothesis on tear film stability. Ophthalmologica 1987;195:119-24.

125. Korb D, Craig J, Doughty M, Guillon J-P, Smith G, Tomlinson A. The Tear Film: structure, function and clinical examination. London: Butterworth-Heinemann, 2002:51-83.

126. Kwok LS. Calculation and application of the anterior surface area of a model human cornea. J Theor Biol 1984;108:295-313.

127. Holly F. Tear film physiology in contact lens wear, Part II, Contact lens tear film interactions. Am J Optom Physiol Opt 1981;58:331-141.

128. Mc Donald J, Brubaker S. Meniscus-induced thinning of tear films. Am J Ophthalmol 1986;72:139.

129. Brubaker RF, Ezekiel S, Chin L, Young L, Johnson SA, Beeler GW. The stress-strain behavior of the corneoscleral envelope of the eye. I. Development of a system for making in vivo measurements using optical interferometry. Exp Eye Res 1975;21:37-46.

130. Guillon J-P. Current clinical techniques to study the tear film. In: Korb D, Craig J, Doughty M, Guillon J-P, Smith G, Tomlinson A, ed. The Tear Film: structure, function and clinical examination. London: Butterworth-Heinemann, 2002:51-83.

131. Korb D. The tear film - its role today and in the future. In: Korb D, Craig J, Doughty M, Guillon J-P, Smith G, Tomlinson A, ed. The Tear Film: structure, function and clinical examination. London: Butterworth-Heinemann, 2002:126-93.

132. Lemp MA. Report of the National Eye Institute/Industry workshop on Clinical Trials in Dry Eyes. CLAO J 1995;21:221-32.

133. Guillon J. Current clinical techniques to study tear film and tear secretions. In: Korb D, Craig J, Doughty M, Guillon J, Smith G, Tomlinson A, ed. The Tear Film. London: ButterworthHeinemann, 2002:52-81.

134. Klein M. The lacrimal strip and the precorneal flim in cases of Sjögren's syndrome. Br J Ophthalmol 1949;33:387-8.

135. Wright P. Diagnosis and management of dry eyes. Trans Ophthalmol Soc UK 1971;91:119-28.

136. Baum JL. Systemic disease associated with tear deficiencies. Int Ophthalmol Clin 1973;13:15784.

137. Mainstone JC, Bruce AS, Golding TR. Tear meniscus measurement in the diagnosis of dry eye. Curr Eye Res 1996;15:653-61.

138. Doughty MJ, Laiquzzaman M, Button NF. Video-assessment of tear meniscus height in elderly Caucasians and its relationship to the exposed ocular surface. Curr Eye Res 2001;22:420-6.

139. Efron N. Tear film dysfunction. In: Efron N, ed. Contact Lens Complications. Oxford: Butterworth-Heinemann, 1999:61-71.

140. Terry JE. Eye disease of the elderly. J Am Optom Assoc 1984;55:23-9.

141. Golding TR, Bruce AS, Mainstone JC. Relationship between tear-meniscus parameters and tearfilm breakup. Cornea 1997;16:649-61.

142. Tomlinson A, Craig J. Time and tear film. In: Korb D, Craig J, Doughty M, Guillon J-P, Smith G, Tomlinson A, ed. The Tear Film: structure, function and clinical examination. London: Butterworth-Heinemann, 2002:83-104.

143. Lemp MA. The mucin-deficient dry eye. Int Ophthalmol Clin 1973;13:185-9.

144. Korb DR, Greiner JV, Herman J. Comparison of fluorescein break-up time measurement reproducibility using standard fluorescein strips versus the Dry Eye Test (DET) method. Cornea 2001;20:811-5.

145. Pflugfelder SC, Tseng SC, Sanabria O et al. Evaluation of subjective assessments and objective diagnostic tests for diagnosing tear-film disorders known to cause ocular irritation. Cornea 1998;17:38-56. 
146. Guillon M, Styles E, Guillon JP, Maissa C. Preocular tear film characteristics of nonwearers and soft contact lens wearers. Optom Vis Sci 1997;74:273-9.

147. Ousler GW, Michaelson C, Christensen MT. An evaluation of tear film breakup time extension and ocular protection index scores among three marketed lubricant eye drops. Cornea 2007;26:949-52.

148. Ponder E, Kennedy W. On the act of blinking. Q J Exp Physiol 1927;18:89-110.

149. Telford C, Thompson N. Some factors influencing voluntary and reflex eyelid responses. J Exp Psychol 1933;16:35-9.

150. Doughty MJ. Consideration of three types of spontaneous eyeblink activity in normal humans: during reading and video display terminal use, in primary gaze, and while in conversation. Optom Vis Sci 2001;78:712-25.

151. Hill RM, Carney LG. The effect of hard lens wear on blinking behavior. Int Contact Lens Clin 1984;11:242-8.

152. Collins M, Heron H, Larsen R, Lindner R. Blinking patterns in soft contact lens wearers can be altered with training. Am J Optom Physiol Opt 1987;64:100-3.

153. Doughty MJ. Further assessment of gender- and blink pattern-related differences in the spontaneous eyeblink activity in primary gaze in young adult humans. Optom Vis Sci 2002;79:43947.

154. Abelson MB, Holly FJ. A tentative mechanism for inferior punctate keratopathy. Am J Ophthalmol 1977;83:866-9.

155. Carney LG, Hill RM. The nature of normal blinking patterns. Acta Ophthalmol (Copenh) 1982;60:427-33.

156. Carney LG, Hill RM. Variation in blinking behavior during soft lens wear. Int Contact Lens Clin 1984;11:250-3.

157. Barbato G, Ficca G, Muscettola G, Fichele M, Beatrice M, Rinaldi F. Diurnal variation in spontaneous eye-blink rate. Psychiatry Res 2000;93:145-51.

158. York M, Ong J, Robbins JC. Variation in blink rate associated with contact lens wear and task difficulty. Am J Optom Arch Am Acad Optom 1971;48:461-7.

159. Pointer JS. Eyeblink activity with hydrophilic contact lenses. A concise longitudinal study. Acta Ophthalmol (Copenh) 1988;66:498-504.

160. Brown M, Chinn S, Fatt I, Harris M. The effect of soft and hard contact lenses on blink rate, amplitude and length. J Am Optom Assoc 1973;44:254-7.

161. Nakamori K, Odawara M, Nakajima T, Mizutani T, Tsubota K. Blinking is controlled primarily by ocular surface conditions. Am J Ophthalmol 1997;124:24-30.

162. Yolton DP, Yolton RL, Lopez R, Bogner B, Stevens R, Rao D. The effects of gender and birth control pill use on spontaneous blink rates. J Am Optom Assoc 1994;65:763-70.

163. Schlote T, Kadner G, Freudenthaler N. Marked reduction and distinct patterns of eye blinking in patients with moderately dry eyes during video display terminal use. Graefes Arch Clin Exp Ophthalmol 2004;242:306-12.

164. Yap M. Tear break-up time is related to blink frequency. Acta Ophthalmol (Copenh) 1991;69:92-4.

165. Collins MJ, Iskander DR, Saunders A, Hook S, Anthony E, Gillon R. Blinking patterns and corneal staining. Eye Contact Lens 2006;32:287-93.

166. Buch J. Peripheral corneal staining: a survey of RGP laboratories. Contact Lens Spectrum 1997;12(7):31-7.

167. Efron N. Blinking. In: Efron N, ed. Contact Lens Complications. Oxford: ButterworthHeinemann, 1999:3-9.

168. Fonn D. Preventing contact lens dropouts. Contact Lens Spectrum 2002;17:43-51. 



\section{CHAPTER 2}

The Art of Rigid Gas Permeable Contact Lens Fitting

"Respect for all" (Nelson Mandela) 


\section{Introduction}

Chapter 2 of this thesis is an introduction to rigid gas permeable lens fitting. The first section of this chapter (part 2.1) tries to answer the question: can we improve comfort of rigid gas permeable lens wear by optimizing the lens fit? And if so, can more sophisticated fitting methods than traditionally used techniques based on keratometry measures, such as corneal topography, be helpful in this respect? The second section (part 2.2) describes how rigid gas permeable lens fitting can be improved. It focuses on strategies to optimally distribute equal lens pressure over the corneal surface. In other words: what are the strategies to create a better lens-tocornea relationship? This section was published as a review article, based on available literature and practical experience, in Optometry in Practice under the title: "Respecting the Shape of the Cornea in Rigid Gas Permeable Lens Fitting". 


\section{1}

\section{Optimizing RGP Lens Fitting in Normal Eyes Using 3D Topographic Data}

Van der Worp E, De Brabander J, Lubberman B, Marin G, Hendrikse F. Contact lens \& Anterior Eye 2002;11:1-5

Eye Research Institute Maastricht, Department of Ophthalmology Academic Hospital, Maastricht University, The Netherlands (EW, JB, FH), Sumipro, Almelo, The Netherlands (BL) and Ocular Sciences (formerly Essilor CL division), R\&D International, Créteil, France (GM) 


\begin{abstract}
Purpose: To analyse, retrospectively, the effect of fitting characteristics on comfort of wear and the role that 3D topographic data can play in attaining an optimal lens fit with rigid gas permeable (RGP) contact lenses in normal eyes.

Methods: Included were 60 normal myopic eyes $(1.00-5.00 \mathrm{D})$ with astigmatism limited to 2.00D. Lenses were ordered empirically, based on traditional fitting rules.
\end{abstract}

Results: The initial fit based on traditional computation was accepted in $40 \%$ of the eyes. To achieve an acceptable fit, $15 \%$ of the eyes needed an adaptation of the back optic zone radius, in $28 \%$ switching from a multicurve to an aspheric lens design was indicated and in $17 \%$ a non-rotational symmetric lens design was favoured. The reason for changing the lens parameters could in $88 \%$ be attributed to mid-peripheral differences between corneal shape as found with 3D corneal topography. Average comfort of wear improved statistically significantly $\left(\chi^{2}, \mathrm{p}<0.05\right)$ from 5.2 initially to 7.7 after 3 months in the group with optimal lens fits, while comfort slightly decreased (not significant) in the group with sub-optimal fits. Switching to a non-rotational symmetric lens design, when indicated, improved comfort significantly $\left(\chi^{2}, \mathrm{p}<0.05\right)$ from 5.0 to 7.3 .

Conclusion: In normal eyes, the measurement of corneal shape, especially in the mid-peripheral regions, is of importance to optimise RGP lens fit. Optimised lens fits increases comfort of wear.

Key words: Rigid gas permeable contact lenses, fitting technique, corneal topography, lens comfort 
Rigid gas permeable (RGP) contact lenses have several advantages over hydrogel contact lenses. One of the most important advantages is their good optical quality. Another benefit is the high oxygen transmissibility, making them suitable for both daily wear (DW) and extended wear (EW). In addition, tear film exchange is superior to that of hydrogel lenses, which is beneficial to oxygen delivery to the cornea ${ }^{1}$ and to removal of tear debris and metabolic waste. ${ }^{2}$

RGP lenses have been proven to be a safer type of lens wear than hydrogel lenses. The far most important contact lens complication, corneal infection, is less frequently reported in RGP lens wear. ${ }^{3-6}$ Cheng et al. ${ }^{6}$ found an incidence of 1.1 per 10,000 cosmetic contact lens wearers in DW RGP and 3.5 in DW hydrogel lens wear in a retrospective study in the Netherlands. For EW with conventional hydrogel lenses the incidence was much higher: 20:10,000. Data on RGP EW were not available. Recent studies showed reduced bacterial binding to corneal epithelial cells in RGP lens wear compared to both conventional hydrogel and hyper-Dk Silicone hydrogel lens wear. ${ }^{7,8}$ In fact, no statistically significant difference in bacterial binding in RGP lens wear was found compared to a (non-lens wearing) control group.7

Another advantage of RGP lenses over hydrogel lenses is deposit resistance. RGP lenses attract fewer deposits than hydrogel lenses although there is a high intermaterial variability.?

Solution allergies and toxic reactions have been reported less frequently in RGP lens wear and contact lens induced papillary conjunctivitis is seen considerably less frequently in RGP lens wear. ${ }^{9,10}$ The possibility to produce a wide variety of lens geometries and lens types (such as bifocal, multifocal and toric designs) as well as the possibility to modify or polish these lenses, constitute some other advantages. ${ }^{9}$ Additional, for some patients better lens handling and maintenance are also essential aspects. Finally, lower costs of RGP lenses and lower costs of maintenance should not be overlooked as factors in patient considerations on lens choice.

However, the ratio between hydrogel and RGP lens use worldwide has been in favour of hydrogel lenses for years. For instance: in the UK in 1999 14\% of lenses worn were rigid, in the US this was close to $10 \% .{ }^{11}$ The results of a survey carried out in 2001 amongst contact lens practitioners (Figure 1) showed that the percentages of RGP lens fits were evidently lower than those for hydrogel lenses both with new fits and refits. ${ }^{12}$ This is also the case in the Netherlands, although the amount of RGP lens fittings is high compared to other countries. This is in conjunction with calculations of $\mathrm{Cheng}^{13}$ and others, who calculated a relatively high amount 
(46.4\%) RGP lens wearers amongst the contact lens population in the Netherlands in 1999. The most important disadvantage of RGP lenses compared to hydrogel lenses is (initial) discomfort. ${ }^{14,15}$ Other disadvantages, especially for the contact lens practitioner, are the more complicated fitting and the need for reusable trial lenses.

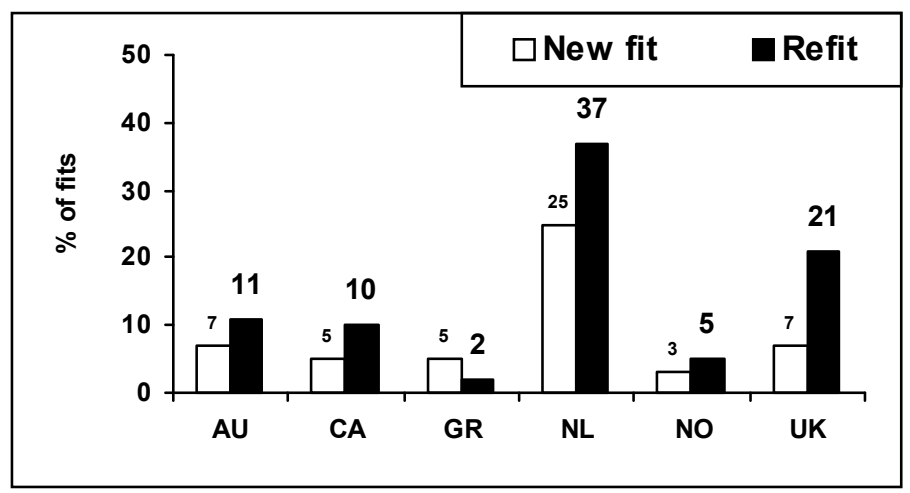

FIGURE 1. Percentages of RGP lens new fits and refits in Australia (AU), Canada (CA), Greece (GR), the Netherlands (NL), Norway (NO) and the United Kingdom (UK).

The purpose of this study is to analyse, retrospectively, the effect of fitting characteristics on comfort of wear and the role 3D topographic data can play in attaining an optimal lens fit with RGP contact lenses in normal eyes.

\section{Methods}

A total of 60 myopic eyes between 1.00 and 5.00D, free from pathological eye conditions and no history of lens wear were included in this study. K-readings ranged between 7.20 and $8.40 \mathrm{~mm}$, and corneal astigmatism was limited to $2.00 \mathrm{D}$. The study was performed in two separate practices in different parts of the Netherlands.

On all eyes 3D topography was performed using the Maastricht Shape Topographer (MST) at the first visit. ${ }^{16}$ Following topography RGP lenses were ordered for all patients using traditional fitting rules based on central K-readings and visual vertical iris diameter (VVID). Randomly, one eye was fitted with a multicurve (MC) and the other eye with an aspheric (AS) lens design. The MC lens was a standard tetra-curve design which back optic zone radius (BOZR) was chosen 0.10 $\mathrm{mm}$ flatter than the flattest central keratometer reading for low corneal astigmatism (0-0.50D), $0.05 \mathrm{~mm}$ flatter in astigmatism of $0.75-1.25 \mathrm{D}$ and in alignment for 
1.50-2.00D astigmatism. The AS design was a lens with a 14 degree spherical central zone and a peripheral zone having an increasing eccentricity towards the periphery of the lens, reaching a total eccentricity of 0.6 at 30 degrees. For all AS lenses the BOZR was chosen $0.05 \mathrm{~mm}$ flatter than the average central keratometer readings. A 11:9 ratio between VVID and total lens diameter was chosen for both lens designs. All lenses were made of Fluoroperm 60 material.

TABLE 1. Grading of tear layer thickness

\begin{tabular}{ll}
\hline Grade & \\
\hline+2 & Rejected thick \\
+1 & Sub-optimal thick \\
0 & Optimal \\
-1 & Sub-optimal thin \\
-2 & Rejected thin \\
\hline
\end{tabular}

TABLE 2. Average tear layer thickness scores in acceptable and rejected fits

\begin{tabular}{lllllll}
\hline & Central & \multicolumn{4}{l}{ Mid-periphery } & Edge \\
& Flat & Steep & Flat & Steep & Flat & Steep \\
\hline Acceptable & 0.1 & 0.1 & 0.2 & 0.3 & 0.4 & 0.6 \\
Rejected & 0.4 & 0.6 & 0.5 & 1.0 & 0.7 & 1.2 \\
\hline
\end{tabular}

The lens fit was evaluated by two masked investigators (the same investigators at both practices) who scored: tear layer thickness in the central and mid-peripheral area and edge lift in both the steepest and flattest meridian using fluorescein dye. The tear layer thickness was graded either optimal, slightly too thick or too thin but acceptable (sub-optimal) or not acceptable (rejected) as shown in Table 1. Diameter, movement and centration were also graded: optimal (0), acceptable (suboptimal, 1) or non-acceptable (rejected, 2). These assessments were performed at three subsequent visits: at dispensing, after 2 weeks of lens wear and 3 months after dispensing. Comfort was scored on a Visual Analogue Scale (VAS) from 0 - 10 for each eye separately at each consequent visit.

Optimising the fits in the rejected group by ordering lenses with new specifications or by changing the lens design was allowed. In case an acceptable (e.g. optimal or sub-optimal) fit in all meridians could not be reached using a MC or AS lens design (showing an non-acceptable toric fluorescein pattern), changing to a nonrotational symmetric lens design (NRS) was permitted. The standard design of this lens included one spherical meridian (steeper appearance) and one aspherical (0.6 eccentricity) meridian (flatter appearance), thus creating a peripheral back toric lens design. 


\section{Results}

Of all initial fits based on traditional computation, $40 \%$ was acceptable (optimal or sub-optimal), $15 \%$ needed an adaptation of the back optic zone radius, $28 \%$ needed change from a MC to an AS lens design and in 17\% a NRS lens design was favoured. In Table 2 the average scores for tear film thickness in acceptable and rejected fits are given for the flat and steep meridians, centrally, mid-peripherally and at the edge. A statistically significant $\left(\chi^{2}, p<0.10\right)$ difference in average tear layer thickness between acceptable and rejected fits in the mid-periphery and at the edge in the steep meridian was found. Comparing this information with the MST topographer data, it was calculated that the reasons for changing the lens parameters originated in $88 \%$ of cases from mid peripheral differences between corneal shape as found with topography and lens shape as predicted from K-readings.

The data on comfort scores at each visit in the group of acceptable fits were related to optimal and sub-optimal fits (Figure 2). At the initial visit the group of suboptimal fits reached a slightly (not statistically significant) higher average score on the VAS (6.1) than the group of optimal fits (5.2). The opposite is true at the two week follow up visit, 7.2 for the optimal group versus 6.3 for the sub-optimal group, and neither statistically significant. After three months the group of optimal fits scored 7.7 on the comfort scale whereas comfort in the sub-optimal group was 5.7, and this difference was statistically significant $\left(\chi^{2}, \mathrm{p}<0.05\right)$. Also the gain in comfort between the initial visit (5.2) and after three months (7.7) in the optimal group was statistically significant $\left(\chi^{2}, \mathrm{p}<0.05\right)$. In the group of sub-optimal fits there was only a very small temporary increase in comfort from dispensing (6.1) till two weeks (6.3), while at three months comfort was even slightly (but not statistically significant) decreased to 5.7 .

To make a retrospective analysis of the changes in comfort possible, the patients were subdivided in three groups on the basis of the lens geometry worn at three months (Figure 3). Comfort at initial visit was not related to the type of lens geometry. Within the AS group comfort increased statistically significantly $\left(\chi^{2}\right.$, $\mathrm{p}<0.05)$ from dispensing (5.6) to two weeks of wear (7.5). Although there was an increase in comfort over that same period for the MC group as well (from 5.9 to 6.7), this was not statistically significant. Both groups did show a statistically significant $\left(\chi^{2}, \mathrm{p}<0.05\right)$ increase in comfort between the initial visit and three months follow up.

In contrast with the other two groups, comfort hardly increased between dispensing (4.8) and after two weeks (5.0) in the group with non-acceptable toric fluo- 
rescein patterns with the standard lens design ( $\mathrm{MC}$ or AS). At this point the lens design was changed to a NRS lens which resulted in an increase in comfort of 2.3 after three months. This increase was statistically significant $\left(\chi^{2}, \mathrm{p}<0.05\right)$ and resulted in a final comfort rate of 7.3 which is equal to the average comfort scores reached with the other two lens designs.

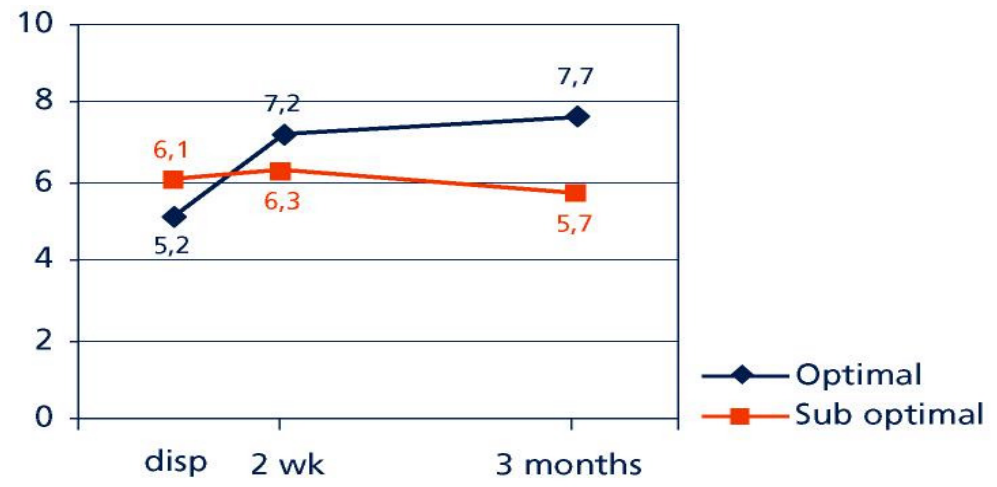

FIGURE 2. Comfort of wear versus lens type in optimal and sub-optimal fits.

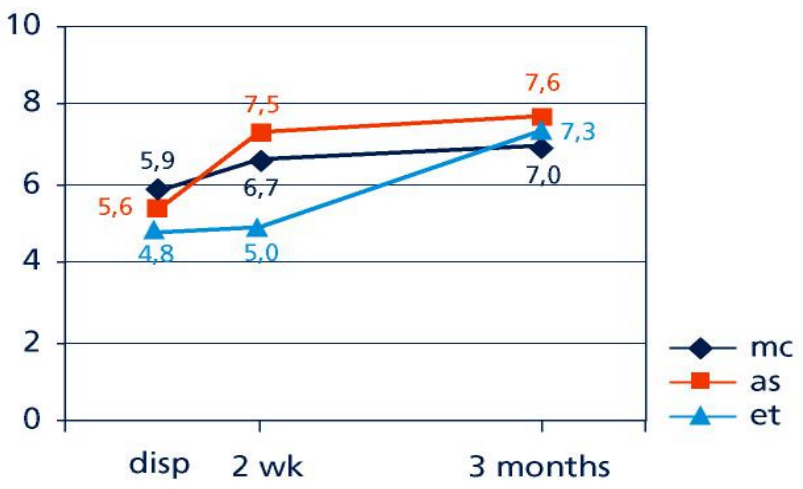

FIGURE 3. Average comfort scores at dispensing, after 2 weeks and three months given per lens type finally worn. 


\section{Discussion}

\section{Fitting Characteristics}

To classify the fit we chose to assess the fluorescein pattern centrally, midperipherally and at the edge in both the steepest and flattest meridian. We realise that manual and subjective grading has some limitations, but up to now no reliable technique for measuring post-lens tear film objectively is available.

The results of this study show that the fitting technique for RGP lenses can be improved compared to the traditional method based on central K-readings. Measuring the corneal shape (especially in the mid-periphery) seems to be of importance to optimise the RGP lens fit in normal eyes. Only $40 \%$ of lenses that were fit traditionally were acceptable. This outcome is inconsistent with the results of previous studies, in which percentages of around $85 \%$ were found. ${ }^{17}$ It is suggested that the criteria applied to evaluate the fit in this study were more critical than in the former studies which might explain this inconsistency.

In $60 \%$ of all eyes a change in lens fit was necessary to obtain an acceptable fit. This finding suggests the need for a trial lens fitting before dispensing lenses when using traditional fitting techniques. The BOZR had to be changed in $15 \%$ of the cases to achieve an evenly distributed fluorescein pattern without unacceptable bearing or pooling and $28 \%$ needed a change from a MC to an AS lens design. This confirms the finding that the shape of the cornea cannot be predicted accurately from central keratometer readings alone. Furthermore, $17 \%$ of the original fits based on central K-readings needed a NRS lens (e.g. a back toric lens design) after evaluation. This figure is surprisingly high since the inclusion criteria excluded corneal astigmatism over $2.00 \mathrm{D}$ (the highest corneal astigmatism found in this study was $1.83 \mathrm{D}$ ) and the amount of central corneal astigmatism that is generally considered to require a back toric lens design is around $2.5 \mathrm{D} .{ }^{18} \mathrm{We}$ can assume that corneal astigmatism in these cases increased towards the periphery. Based on this we can say that a toric fluorescein pattern which fails to provide an acceptable physiological fit is a more important indication for a toric back surface geometry than central corneal astigmatism.

With regard to the value of $3 \mathrm{D}$ corneal topography in fitting RGP lenses, it can be concluded that in $88 \%$ of the cases necessary changes of lens parameters resulted from mid-peripheral differences between corneal shape as assessed by topography and lens shape as predicted from K-readings. Collecting more information about the shape of the cornea could therefore be of high value when fitting rigid contact lenses in normal eyes. This information is encouraging for the development of cus- 
tom made aspheric and asymmetric contact lens geometries based on corneal topography. In addition, custom made lenses could promote RGP lens prescribing because disadvantages such as the use of trial lenses and the need for specialised fitting skills are reduced.

\section{Comfort}

An important clinical question is whether comfort of wear can be improved by fitting a lens more accurately to the shape of the cornea. In our study comfort of optimal and sub-optimal fits did not improve significantly between dispensing and two weeks. One may suggest that anaesthetics ${ }^{19}$ or other eye drops ${ }^{20}$ might be used to decrease initial discomfort.

Comfort did improve over a period of three months in the optimal fit group but not in the sub-optimal group. The difference after three months between these two groups was 2 on a 10 point scale, which is a statistically significant difference. This information is of interest to the practitioner, since it implies that even small improvements in RGP fits influence comfort of wear significantly. It should also be noted that this could potentially lead to drop out among patients with acceptable but not optimal lens fits.

An interesting result of this study is that NRS lens designs improved comfort significantly. Practitioners should be aware that some eyes with lesser degrees of central corneal astigmatism can show toric fluorescein patterns because of an increase of corneal astigmatism towards the periphery and would therefore benefit from NRS lens designs.

\section{Conclusions}

Based on the results of this study we can conclude that fitting techniques can be improved by making use of $3 \mathrm{D}$ topographic corneal data and that in the long term good fitting characteristics do significantly improve comfort of RGP lens wear in normal eyes. This information is encouraging for the development of custom made aspheric and asymmetric contact lens geometries.

\section{Acknowledgements}

This study was supported by a grant from the EC as part of BRITE-EURAM project BE 97-4608. We thank Polymer Technology Corporation (PTC) for supply of the materials. Special thanks go to Ziemerink Totaal Optiek in Glanerbrug and Groenhof Optometrie in Amstelveen for their help and access to their practices. Also thanks to Ineke Krijger of the Hogeschool van Utrecht, the Netherlands for her valuable comments. 


\section{References}

1. Burger R. Effect of contact lens motion on the oxygen tension distribution under the lens. Am J Optom Physiol Opt 1974;57:441.

2. Key J, 2nd. Are hard lenses superior to soft? Arguments in favor of hard lenses. Cornea 1990;9 Suppl 1:S9-11; discussion S5.

3. Chalupa E, Swarbrick HA, Holden BA, Sjostrand J. Severe corneal infections associated with contact lens wear. Ophthalmology 1987;94:17-22.

4. Poggio EC, Glynn RJ, Schein OD et al. The incidence of ulcerative keratitis among users of daily-wear and extended-wear soft contact lenses. N Engl J Med 1989;321:779-83.

5. Nilsson SE, Montan PG. The annualized incidence of contact lens induced keratitis in Sweden and its relation to lens type and wear schedule: results of a 3-month prospective study. CLAO J 1994;20:225-30.

6. Cheng $\mathrm{KH}$, Leung SL, Hoekman $\mathrm{HW}$ et al. Incidence of contact-lens-associated microbial keratitis and its related morbidity. Lancet 1999;354:181-5.

7. Ladage PM, Yamamoto K, Ren DH et al. Effects of rigid and soft contact lens daily wear on corneal epithelium, tear lactate dehydrogenase, and bacterial binding to exfoliated epithelial cells. Ophthalmology 2001;108:1279-88.

8. Ren DH, Yamamoto K, Ladage PM et al. Adaptive effects of 30-night wear of hyper-O2 transmissible contact lenses on bacterial binding and corneal epithelium: a 1-year clinical trial. Ophthalmology 2002;109:27-39.

9. Phillips A. Rigid gas permeable and hard corneal lens fitting. In: Phillips A, Stone J, ed. Contact Lenses. London: Butterworth, 1989:333-81.

10. Efron N. Papillary Conjuntivitis. In: Efron N, ed. Contact Lens Complications. Oxford: Butterworth-Heinemann, 1999:40-7.

11. Loan N. Psychological, clinical and commercial perspectives on contact lenses: invisible, reliable, profitable. Cont Lens Anterior Eye 2000;23:36-43.

12. Van der Worp E, Morgan P, Efron $\mathrm{N}$ et al. Enquete contactlensaanpassingen internationaal 2001. VISUS 2001;1:27-31.

13. Cheng K. Contact lens wear and its complications. PhD thesis. Amsterdam: University of Amsterdam; 1999.

14. Yamane SJ. Are hard lenses superior to soft? The advantages of soft lenses. Cornea 1990;9 Suppl 1:S12-4; discussion S5.

15. Fonn D, Gauthier CA, Pritchard N. Patient preferences and comparative ocular responses to rigid and soft contact lenses. Optom Vis Sci 1995;72:857-63.

16. Jongsma FH, De Brabander J, Hendrikse F, Stultiens BA. Development of a wide field height eye topographer: validation on models of the anterior eye surface. Optom Vis Sci 1998;75:6977.

17. De Brabander J, Kok JH, Nuijts RM. Evaluation of a new system of lens parameter selection and comparison of traditional vs one-step lens care systems for aspheric high-Dk RGP contact lenses. CLAO J 2000;26:193-9.

18. Grosvenor T. Fitting the astigmatic patient with rigid contact lenses. In: Ruben M, Guillon M, ed. Contact lens practice. London: Chapman \& Hall, 1994:623-47.

19. Bennett ES, Smythe J, Henry VA et al. Effect of topical anesthetic use on initial patient satisfaction and overall success with rigid gas permeable contact lenses. Optom Vis Sci 1998;75:800-5.

20. Jackson R, Westin E, Mathur R et al. The influence of Voltaren on ocular discomfort associated with adaptation gas permeable contact lenses. Contact Lens Spectrum 2001;16:3. 


\section{2}

\section{Respecting the Shape of the Cornea in Rigid Gas Permeable Lens Fitting}

Van der Worp E.

Optometry in Practice 2004;5:153-162

Eye Research Institute Maastricht, Department of Ophthalmology Academic Hospital, Maastricht University, The Netherlands 


\begin{abstract}
The primary goal in fitting RGP lenses is to respect the shape of the cornea as much as possible and to distribute pressure equally over the entire corneal surface. If the surface area of contact is maximised, the weight of the lens is distributed over the largest possible area of the cornea. Corneal topographers and modern, sophisticated lathing techniques make it possible to fit RGP lenses more accurately, which will better follow the shape of the cornea. This may result in less epithelial indentation and corneal warpage, but also can lead to improved comfort and may help reduce common RGP lens problems such as 3- and 9-o'clock staining. A systematic approach in evaluating the RGP lens on the eye is important to bring the technical and physiological benefits of RGP lenses to clinical success.
\end{abstract}

Key words: Rigid gas permeable contact lenses, fitting techniques, corneal shape, corneal astigmatism 
On paper, it is easy to list the many advantages that rigid gas-permeable (RGP) lenses have to offer over hydrogel contact lenses. One of their most important advantages is their good optical quality, correcting higher-order aberrations, ${ }^{1}$ since they mask all irregularities of the corneal surface. Another benefit of RGP lenses is their high oxygen transmissibility, making them suitable for both daily-wear (DW) and extended-wear (EW) modalities. In addition, tear film exchange is superior to that of hydrogel lenses, which is beneficial for oxygen delivery to the cornea ${ }^{2}$ and for removal of tear debris and metabolic waste. ${ }^{3}$

In general, RGP lenses have been proven to be a safer type of lens than hydrogel lenses. The most serious contact lens complication, corneal infection, is reported less frequently in RGP lens wear than in other modalities. ${ }^{4-6}$ In a retrospective study in the Netherlands Cheng et al. ${ }^{7}$ found an incidence in cosmetic contact lens wearers of 1.1 per 10000 in DW RGP lens wear and 3.5 per 10000 in DW hydrogel lens wear. For EW with conventional hydrogel lenses the incidence was much higher - 20 per 10 000. Data on RGP EW are not available. Recent studies have shown that bacterial binding to corneal epithelial cells is reduced in RGP lens wear compared to conventional hydrogels, but also compared to hyper- $D k$ silicone hydrogel lens wear. ${ }^{8,9}$ In fact, no statistically significant difference in bacterial binding was found between wearers of normal RGP lenses and a (non-lens-wearing) control group.

Another advantage of RGP lenses over hydrogel lenses is deposit resistance. RGP lenses attract fewer deposits than hydrogel lenses, although there is a high intermaterial variability. ${ }^{10}$ Solution allergies and toxic reactions are reported less frequently in RGP lens wear and contact lens-induced papillary conjunctivitis is seen considerably less frequently in RGP lens wear than in hydrogel lens wear. ${ }^{11}$ The ability to produce a wide variety of lens geometries and lens types (such as bifocal, multifocal and toric designs) as well as the ability to modify or polish these lenses constitute some other advantages. Additionally, for some patients simpler care and maintenance are essential aspects. Finally, the lower costs of RGP lenses as well as lower costs of maintenance should not be overlooked as factors in patient consideration on lens choice.

In the Netherlands about $10-11 \%$ of the population wears contact lenses; this is relatively high compared to other European countries (percentages of total contact lens population are estimated to be $2.5 \%$ for France and 5\% for the UK and Germany). Based on information from the industry plus an annual consumer survey, ${ }^{12}$ it is estimated that about $39 \%$ of that group is wearing RGP lenses. This percentage has been stable over the last few years. One could speculate whether there exists 
a correlation between the popularity of RGP lens wear and the total number of lens wearers in the Netherlands.

The annual Eurolens research survey ${ }^{13}$ provides comparable data about new fits and refits around the world. On average, $12 \%$ of new fits is with RGP lenses. Exceptions are the Netherlands (24\%) and Japan (21\%). We need to be careful in interpreting these data. In the UK, for instance, only $8 \%$ of new fits are with RGP lenses but $20 \%$ of all refits are with RGP lenses. In the Netherlands $30 \%$ and in Japan $44 \%$ of all refits are with RGP lenses. In all countries, over $50 \%$ of all fits are refits.

Obviously, RGP lenses are not the number-one choice for all patients. When deciding whether to fit RGP lenses or hydrogel lenses, often the complexity of the fitting is mentioned as a major disadvantage of RGP lenses, as well as comfort problems. It is a misconception that RGP lenses are difficult to fit. In fact, following the shape of the cornea to create an optimal and comfortable fit has never been easier thanks to the increasing availability of modern equipment such as corneal topographers and sophisticated lathes.

\section{Respecting the Shape of the Cornea}

\section{Shape}

The primary goal in fitting RGP lenses is to respect the shape of the cornea as much as possible and to distribute pressure equally over the entire corneal epithelium. If the surface area of contact is maximised, the weight of the lens is distributed over the largest possible area of cornea. ${ }^{14}$ In this situation the force per unit of surface area applied to the cornea by the lens is minimised, and the likelihood of corneal distortion is reduced.

It has been shown that lenses that are fitted $0.3 \mathrm{~mm}$ steeper than the flattest meridian induce corneal steepening after short-term wear. ${ }^{15}$ Although the authors state that corneal moulding is the primary factor implicated in the change of shape, corneal edema and post-lens tear film pressures are thought to modify this response. In a recent orthokeratology study, patients showed significant central corneal flattening $(-0.61 \pm 0.35 \mathrm{D} ; \mathrm{p}=0.014)$ within 10 minutes of open-eye lens wear, showing the vulnerable nature of the epithelium and the speed with which it can be altered. 16

Alterations of corneal topography in RGP contact lens wearers have been reported by many researchers. ${ }^{17-19}$ The resting position of the lens on the cornea seems to 
play an important role; the topography of warped corneas is usually characterised by a relative flattening of the cornea underlying the RGP contact lens in its resting position. Lenses that ride high, for example, produce flattening superiorly and result in a relatively steeper contour inferiorly. ${ }^{20}$

Recently, the impact of contact lenses on the cornea has become clear within the framework of laser refractive surgery. ${ }^{21,22}$ RGP lens wearers frequently need to stop wearing their lenses for many weeks before surgery for the cornea to return to its baseline shape. It is becoming increasingly clear that RGP lenses can significantly influence corneal topography. One refractive surgery centre in the Netherlands reported that $95 \%$ of all retreatments for refractive reasons were performed on previous RGP lens wearers. ${ }^{23}$ Currently, we advise RGP-wearing patients to stop wearing their lenses for 8 weeks before laser refractive surgery, and after these 8 weeks to evaluate the cornea at 2 -week intervals until the topography is stable $(0.5 \mathrm{D}$ change or less). Work by Wang et al. ${ }^{22}$ supports this approach; the mean recovery time for corneal warpage in RGP lens wearers was $8.8 \pm 6.8$ weeks in their study. Backsurface aspheric-type multifocal lenses are fitted steeper than the flattest $k$ to create a reading addition towards the periphery of the lens (sometimes up to 3D steeper). This can easily create corneal warpage and when these lenses are fitted this should be kept in mind.

The goal in normal RGP lens wear is to avoid the induction of corneal change as much as possible. In order to do so, knowledge of the shape of the cornea is essential. By far the best instrument to analyse this shape is the corneal topographer. Several attempts to modify manual keratometers to carry out this analysis have been proven to be of limited use. Although many of the Placido disc systems are not able to measure curves in the far periphery of the cornea, they are usually able to measure until the area where the edge of the lens will be positioned. Most corneal topographers extrapolate the 'missing' parts of the cornea that could not be measured, but these data should be interpreted with care, since assumptions are made about the shape of the cornea that do not necessarily reflect the real corneal shape.

The shape of the corneal surface is usually described as a prolate ellipse, indicating a gradual flattening from centre to periphery. This is one of first things that is obvious when looking at an average corneal topography map: relatively cooler colours in the periphery of the corneal map represent this flattening. The amount of flattening is traditionally designated as eccentricity, mostly noted as the $e$-value. The $e$ value of an ellipse can be calculated from the central curvature and the peripheral curvature plus the distance (angle) from the centre where that peripheral curve was 
measured. The average cornea has been described by many and its $e$-value is thought to be somewhere between 0.40 and $0.57 . .^{24-26}$ The $e$-value varies widely among individuals, and ideally it should be measured and evaluated on every single eye before fitting contact lenses. As an example, the Ohio State University looked at the corneal shape of 683 children's eyes (aged 8-15): the vast majority flattened towards the periphery, but two corneas actually steepened. ${ }^{27}$ There seems to be a small correlation between ametropia and eccentricity (higher myopia, reduced eccentricity). ${ }^{24,28}$

Not all meridians of the cornea have the same $e$-value. Most corneal topographers and automated keratometers will provide the average value of all meridians, although some will give $e$-values per meridian or quadrant. A major drawback with regard to $e$-value measurement is that manufacturers of topographers are very secretive about the way they calculate the $e$-value: neither the distance from the centre nor the meridian used are usually revealed, which means that differences in $e$-values between topographers may occur. Unfortunately there is no standard for defining corneal shape.

Besides this, another disadvantage of the $e$-value is that it can only describe prolate shapes. In corneas that are steeper in the periphery by default or that are reshaped that way by orthokeratology or laser surgery, an oblate shape is present. In these cases the $e$-value is useless, since mathematically it can only define shapes larger than zero (spherical). This problem can be overcome by using what has been called the $p$-value, which can be derived directly from the $e$-value: $p=1-e^{2}$. By using the $p$-value the exact same shape is described, but it should be borne in mind that a circle's value is now 1 instead of 0.29 The $p$-value of all prolate shapes is less than 1 and in the case of peripheral steepening, the $p$-value is larger than 1 , which is exactly the opposite of the $e$-value. Practitioners may be confronted with $p$-values as an alternative to $e$-values in the international literature, and should be aware of the opposite effect compared to the $e$-value.

Another approach to describe the asphericity of the cornea is to use $Q$. This value can be derived: $Q=p-1$, or $Q=-e^{2}$. A negative $Q$-value describes prolate shapes and positive values an oblate shape, but the $Q$-value of a sphere remains 0 , the same as the $e$-value. ${ }^{30,31}$ Therefore the $Q$-value has advantages over both the other shape descriptors and may be considered as a standard index for describing corneal shape, ${ }^{32}$ especially in orthokeratology and refractive surgery.

In reality, however, the actual corneal shape is not as easily defined as a standard ellipse. Especially towards the periphery it becomes more complex and less predict- 
able. The corneal shape is usually more spherical near the apex and it may flatten at a variable (usually progressive) rate towards the periphery. Zernike polynomials are often used to describe the corneal shape in more detail. But even these complex mathematical formulations have their limitations and newer mathematical definitions of the corneal shape are being developed. However, the clinical usefulness of these complex formulations when manually fitting RGP lenses is very limited. Therefore, to describe corneal eccentricity, shape parameters are still preferred and provide a good idea about the flattening of the cornea. The $e$-value is still the parameter most used in contact lens practice and most topographers and automated keratometers currently use this value.

As a rule of thumb, practitioners may square the $e$-value that was provided by the topographer or automated keratometer to have some idea of the amount of flattening in the periphery of average corneas. An $e$-value of 0.4 means that the flattening in the periphery at $30^{\circ}$ from the centre is about $0.16 \mathrm{~mm}$. An $e$-value of $0.6 \mathrm{de}$ scribes a flattening of $0.36 \mathrm{~mm}$ and a cornea with an $e$-value of 0.8 is $0.64 \mathrm{~mm}$ flatter in the periphery. From this it can be concluded that, as the $e$-value goes up, flattening increases progressively. This also means that small $e$-values are clinically of little importance, but the importance of $e$-values increases rapidly as they get higher.

To respect the shape of the cornea, the amount of flattening should be followed as closely as possible. A spherical lens (tricurve, tetracurve, etc) on an aspheric cornea could give friction in the mid-periphery of the cornea. Young ${ }^{33}$ describes the percentage of corneal alignment (PCA) when an aspheric and a spherical lens are placed on an average cornea with an $e$-value of 0.45 . When compared to each other, the PCA of an aspheric lens is significantly higher and thus is supporting an even distribution of pressure on the corneal epithelium. A spherical lens on such an (average) aspheric cornea is shown in Figure 1. As most corneas flatten towards the periphery, most lenses should also be aspheric in nature. ${ }^{14,34}$ In practice this usually means choosing a lens with the same $e$-value as the cornea or an $e$-value closest to the nearest flattest value available. Usually aspheric RGP lenses are manufactured with an $e$-value between 0.4 and 0.8 for normal corneas, and higher $e$-values for keratoconic eyes. The common interval between $e$-values of available lenses is 0.15 or 0.2 , but some manufacturers allow the practitioner to order the $e$-value in 0.05 steps if desired.

This way, the shape of the lens is followed accurately in an annular fashion. Some lens design software programs allow the practitioner to choose different $e$-values for different zones. In this way the shape of the cornea can be followed even more 
closely (since the cornea is not usually a perfect ellipse). In Figure 2 an evenly distributed fluorescein pattern is presented, indicating an alignment between the back-surface of the contact lens and the shape of the cornea.

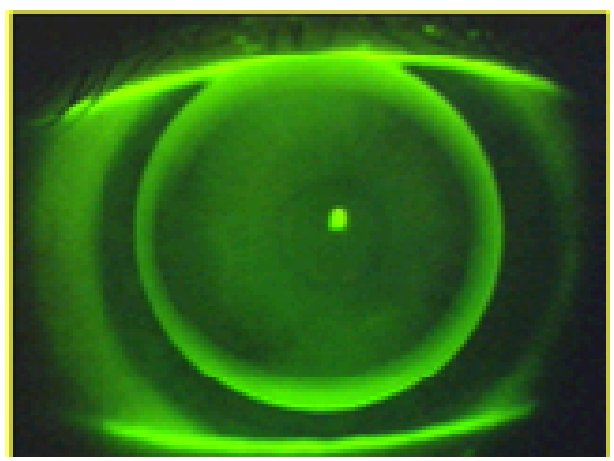

FIGURE 1. A spherical lens on an aspheric cornea: note the mid peripheral bearing.

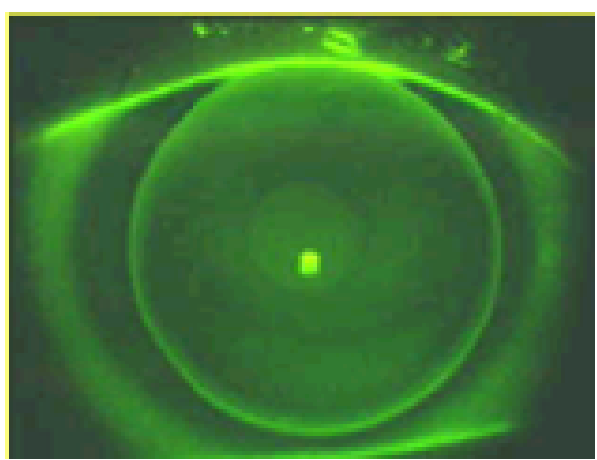

FIGURE 2. An evenly distributed fluorescein pattern indicating an alignment between the back-surface of the contact lens and the shape of the cornea.

In RGP lens wear, 3- and 9-o'clock staining is one of the most often reported problems and this complication is difficult to remedy. Several author $s^{35-38}$ have suggested that in theory an aspheric lens design could be beneficial in managing 3and 9-o'clock staining. Firstly, this is because aspheric back-surface designs may follow the corneal shape closely, lessening areas of contact between the lens and (peripheral) cornea and thus enhancing tear fluid exchange and corneal wettability. Furthermore aspheric lens designs are able to minimise lid-lens interaction, thereby decreasing discomfort and interference with blinking habits. Also, decreased edgelifts in aspheric lenses could lead to a reduction of the bridge effect (upper eyelid bridging the gap between lens edge and cornea) and tear meniscus formation around the edge of the lens ${ }^{39}$ - all thought to be beneficial in 3- and 9-o'clock staining management.

Surprisingly, there are few data available in the literature with regard to the effect of aspheric lenses and 3- and 9-o'clock staining. De Brabander et al..$^{40}$ found no significant difference in 3- and 9-o'clock staining between aspheric and spherical design lenses, but the group size was fairly small (30 patients). Ames $\&$ Erickson ${ }^{41}$ compared spherical (bicurve) and aspheric lens types in 10 patients to study comfort and centration and found that the aspheric lens design resulted in a more central vertical position and were more comfortable. Good central lens position has proven to be beneficial in 3- and 9-o'clock staining management. However, lens design (a simple bicurve spherical design) and manufacturing variables (different 
laboratories were used for different lens types) could also have contributed to this difference. Edwards 14 also mentioned more central lens positioning of lenses with aspheric back-surface geometries, based on manufacturers' claims. In the Jones et al. ${ }^{38}$ nomogram for 3- and 9-o'clock staining management, aspheric back-surface geometry is advised to create a superiorly or centrally positioned lens and/or to create optimal edge lifts.

To summarise, to avoid the induction of corneal topographical changes by RGP lenses, the shape of the cornea should be followed as closely as possible. Matching the corneal shape with the lens shape by using aspheric lenses can be helpful in achieving this and in addition may aid in the management of 3-and 9-o'clock staining. Corneal topography is very useful for this purpose, although the instruments that are currently available have their limitations.

\section{Astigmatism}

The other major factor with regard to respecting the shape of the cornea is corneal toricity. Dealing with corneal toricity follows the same principle as dealing with corneal shape: the lens pressure should be evenly distributed over the corneal surface. Non-toric lenses on with-the-rule corneas will create pressure in the horizontal meridian.

Devising a general rule for the degree of corneal toricity that should be fitted with a toric back-surface is not easy. Textbooks generally consider that toric designs are indicated when the corneal toricity is $2.5-3.0 \mathrm{D}$ or more. ${ }^{42}$ However, this could easily lead to topographical changes and spectacle blur. As stated above, $0.3 \mathrm{~mm}$ steep lenses (accounting for a $1.5 \mathrm{D}$ steep fit) will give significant corneal changes. ${ }^{15}$ In addition to this, corneal toricity may increase or decrease towards the periphery and thus influence fitting characteristics. Szczotka et al. ${ }^{43}$ found peripheral corneal toricity to be one of the major factors determining the success of toric hydrogel lens fitting. Since RGP lenses rest mostly peripherally, this influence should not be neglected either in RGP lenses. Central corneal astigmatism is easier to deal with than limbal to limbal corneal astigmatism when fitting RGP lenses. Corneal topographers can aid in assessing the degree of peripheral astigmatism (Figure 3). If not available, a standard non-toric trial lens can be placed on the eye and the fluorescein pattern will tell the practitioner how much corneal toricity is present and whether this is acceptable or not. 

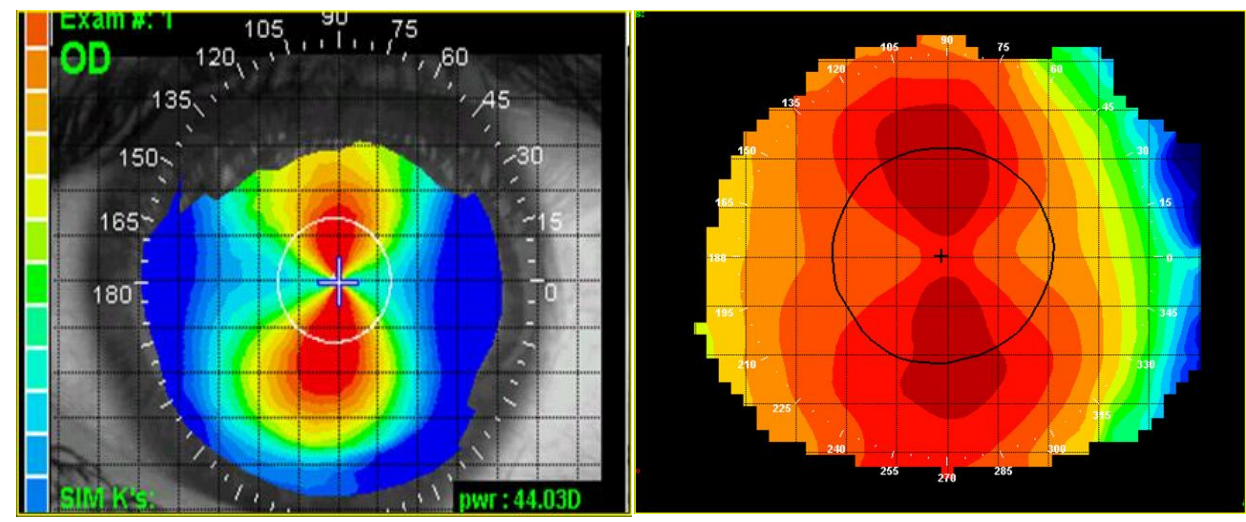

FIGURE 3. Central (left) versus peripheral (right) corneal astigmatism

When significant corneal toricity is noted, the first option is often to choose a full back toric lens design. The flattest meridian is usually fitted in alignment with the cornea or slightly flatter, taking radius of curvature and the $e$-value into account. The other meridian is generally fitted flatter than alignment to create a lens that moves well, but also to compensate for the difference in refractive index between tears and lens material and thus to prevent induced astigmatism. A popular rule of thumb is to take two-thirds of the corneal astigmatism and add this to the flattest meridian to calculate the steepest meridian. However, with the highly sophisticated lathing technology currently available it is technically no problem to compensate the induced astigmatism on the front surface of the lens. Hence, practitioners should not worry about induced astigmatism when fitting the lens. Still, a slightly flatter back optic zone radius (BOZR) in the steepest meridian than alignment is desirable to promote movement, but should be limited to about $0.75 \mathrm{D}$.

Newly developed peripheral or edge toric back-surface geometries with one spherical and one aspheric meridian can be used in lower degrees of corneal toricity; in particular when peripheral corneal toricity is present. Usually one meridian is fitted with a low or zero $e$-value, while the flattest meridian is fitted with an $e$-value between 0.6 and 0.8 . Practical tips are to use fairly high $e$-values that provide more flattening and therefore more peripheral toricity. Also; use large diameters since the toric effect increases towards the periphery. Be aware that, when evaluated with a radiuscope, these lenses are spherical centrally and only start to diverge towards the periphery. Some corneal topographers provide a lens holder, to be placed on the chin rest, to evaluate contact lens surfaces. However, most topographers use assumptions about the shape of the cornea and, since the back-surface of a contact lens is hollow in contrast to the convex corneal shape, this could lead to erroneous values. The lenses are marked in the flattest meridian to make evaluation of the 
position of the lens on the eye possible; these lenses should show no or limited signs of rotation during lens wear.

In managing 3- and 9-o'clock staining, the toric cornea should also be respected. With-the-rule corneal toricity could give rise to mechanical damage of the peripheral corneal epithelium by the lens. A non-toric back-surface geometry lens on such a cornea creates friction in the three and nine o'clock regions of the peripheral cornea and, according to Businger et al ${ }^{44}$ a lens with a toric back-surface geometry should be fitted in these cases. Jones $\&$ Jones ${ }^{45}$ also urge the use of a toric backsurface design in the presence of significant corneal toricity. With toric lens designs, the corneal contour is followed much more precisely and lid-lens interaction can be minimised. In this way, the influence on blinking habits is supposedly diminished, which can be beneficial in the management of 3- and 9-o'clock staining. ${ }^{38,46,47}$

Another reason for using back-surface toric geometries on toric corneas is that this improves lens centration. Especially in with-the-rule toricity there is a tendency for the lens to ride high or low. ${ }^{42,48}$ The latter should be avoided at all times to prevent 3- and 9-o'clock staining.

In summary, to reduce the influence of the contact lens on the corneal epithelium, corneal toricity should be respected. Textbooks generally advise practitioners to use back toric lens designs on corneas with an astigmatism of $2.5 \mathrm{D}$ or $3 \mathrm{D}$ and higher, but this could easily lead to topographical corneal changes and spectacle blur. When fitting back toric lens designs, the amount of peripheral astigmatism should also be taken into account. Different types of back toric surface geometries are available to the practitioner to respect the shape of the cornea at all times. This could also have a positive effect on 3- and 9-o'clock staining management.

\section{Fluorescein Pattern Evaluation}

Evaluation of the lens fit with fluorescein has been used ever since rigid lenses came on to the market. It has been proven to be very useful in clinical practice, although the human eye is only able to detect fluorescein layers with a thickness of at least $20 \mu \mathrm{m} .{ }^{49,50}$ This means that tear layers thinner than $20 \mu \mathrm{m}$ appear dark. For this reason it is difficult to fit orthokeratology lenses relying on fluorescein patterns alone. ${ }^{51}$ With yellow barrier filters the contrast of the fluorescein pattern can be significantly enhanced. The application of these filters combined with different slit lamp techniques (anterior view plus cross-section approach) is highly recommended to gain as much information about the lens fit as possible. 
For an accurate assessment of the lens fit, the tear layer thickness should be assessed centrally, mid peripherally and at the edge of the lens in both the steepest and flattest meridian. This is often mentioned as a complex and difficult component of RGP lens fitting. In fact, it is not difficult if done systematically and using the proper instrumentation. To explain such a systematic evaluation of RGP lens fit, Table 1 shows a grading scale that has been developed for use in both research and teaching. The tear layer thickness is graded as either optimal (0), slightly too thick or too thin but acceptable (grade 1 or suboptimal) or not acceptable (grade 2 or rejected). The diameter and movement of the lens are graded as optimal (0), acceptable (suboptimal, 1) or unacceptable (rejected, 2). Centration only has negative values (there is no such thing as a 'too good' centration), and zero for a perfect centration.

TABLE 1. Grading of tear layer thickness

\begin{aligned} & \hline Grade \\ & \hline+2 Rejected thick \\ &+1 Suboptimal thick \\ & 0 Optimal \\ &-1 Suboptimal thin \\ &-2 Rejected thin \\ & \hline\end{aligned}

This system was used in a study initiated by the University of Maastricht in the Netherlands looking at the relation between RGP lens fitting and comfort of wear. The first question this study sought to answer was what percentage of lens fits were acceptable when only central $k$-readings were used to fit the lenses. The second question in this study was related to corneal topography: can information derived from this technique be beneficial in finding the optimal lens fit? Finally, does accurate RGP lens fitting improve comfort of wear? A total of 60 myopic eyes (myopia between 1.00 and 5.00D), free from pathological eye conditions and with no history of lens wear were included in this study ${ }^{52}$ to answer these questions. $k$-readings ranged from $7.20 \mathrm{~mm}$ to $8.40 \mathrm{~mm}$, and corneal astigmatism was limited to 2.00D. The study was performed in two separate practices in different parts of the Netherlands by two masked investigators.

On all eyes three-dimensional topography was performed at the first visit, using the Maastricht shape topographer. ${ }^{53}$ Following topography, RGP lenses were ordered for all patients using traditional fitting rules based on central $k$-readings and visual vertical iris diameter (VVID). Randomly, one eye was fitted with a multicurve (MC) and the other eye with an aspheric (AS) lens design. The MC lens was a standard tetracurve design. The BOZR of the lens was chosen $0.10 \mathrm{~mm}$ flatter 
than the flattest central keratometer reading when low corneal astigmatism (0$0.50 \mathrm{D}$ ) was present, $0.05 \mathrm{~mm}$ flatter in astigmatism of $0.75-1.25 \mathrm{D}$ and in alignment for $1.50-2.00 \mathrm{D}$ of astigmatism. The AS design was a lens with a $14^{\circ}$ spherical central zone and a peripheral zone in which eccentricity increased towards the periphery of the lens, reaching a total $e$-value of 0.6 at $30^{\circ}$ (based on average corneal $e$-values). For all AS lenses the BOZR was chosen $0.05 \mathrm{~mm}$ flatter than the average central keratometer readings. A 11:9 ratio between VVID and total lens diameter was chosen for both lens designs. All lenses were made of Fluoroperm 60 material.

Of all initial fits based on traditional computation, only $40 \%$ were acceptable (optimal or suboptimal). From the unacceptable fits, $15 \%$ needed an adaptation of the BOZR to be acceptable. In $28 \%$, it was necessary to switch from a MC to an AS lens design to create an acceptable fit, and in $17 \%$ a toric back-surface was necessary since the fluorescein pattern happened to be too toric. This was despite the fact that the maximum degree of central corneal astigmatism was only $1.83 \mathrm{D}$. The influence of peripheral astigmatism on lens fit was evident in these cases.

When the changes in lens fit that were made based on keratometry alone are compared with the topographical data, it can be concluded that in $88 \%$ of cases the reasons for changing the lens parameters originated from mid peripheral differences between corneal shape as established with topography and lens shape as predicted from $k$-readings. In other words, in $88 \%$ of cases the lens fit could have been optimised before fitting by making use of corneal topography data.

The final and most important question the study had to answer was: does accurate lens fitting improve comfort? Figure 4 shows the comfort scores for optimal versus suboptimal fits. At the initial visit and at 2 weeks, there was no statistically significant difference between the two groups. In other words, initially (up to 2 weeks) the accuracy of the lens fit is not important for wearing comfort.

However, after 3 months of lens wear, the group of optimal fits scored 7.7 on the comfort scale whereas comfort in the suboptimal group was 5.7. This difference was statistically significant $\left(\chi^{2}, \mathrm{p}<0.05\right)$. Also the gain in comfort between the initial visit (5.2) and the visit 3 months later (7.7) was statistically significant $\chi^{2}$, $\mathrm{p}<0.05)$ in the group of optimal fits. In the group of suboptimal fits there was only a small temporary increase in comfort from dispensing (6.1) to the visit 2 weeks later (6.3), while after 3 months comfort had even slightly (but not statistically significantly) decreased to 5.7 . 


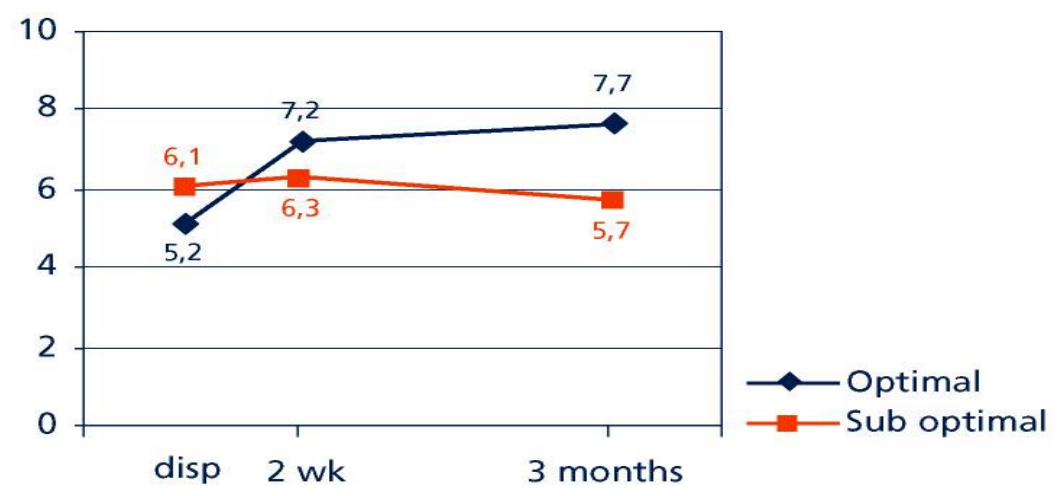

FIGURE 4. Comfort scores for optimal versus suboptimal fits.

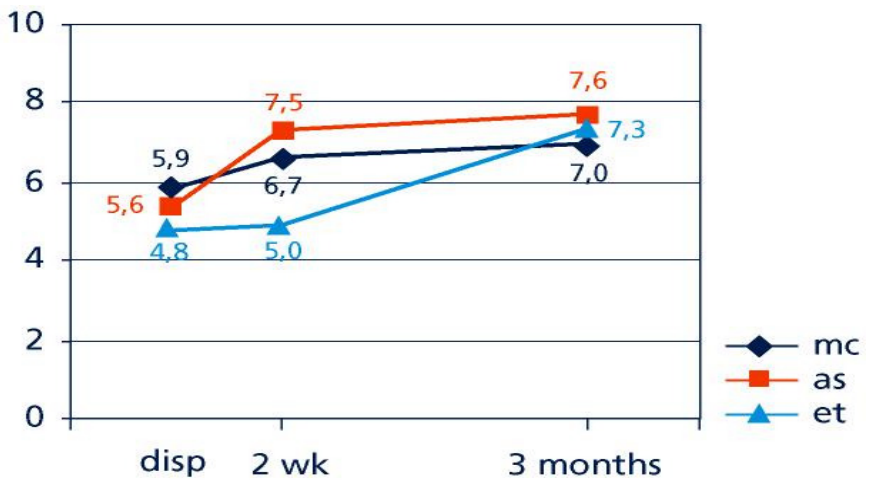

FIGURE 5. Comfort scores for a multicurve (MC), aspheric (AS) and edge toric (ET) lens design.

To analyse the aspect of comfort further, patients were subdivided into three groups on the basis of the lens geometry worn at 3 months (Figure 5). No relationship between comfort and lens geometry was found at the initial visit. Within the AS group, comfort increased significantly $\left(\chi^{2}, \mathrm{p}<0.05\right)$ from dispensing $(5.6)$ to the follow-up visit after 2 weeks of wear (7.5). Although there was also an increase in comfort over that same period in the MC group (from 5.9 to 6.7), this increase was statistically not significant. Both groups did show a significant $\left(\chi^{2}, \mathrm{p}<0.05\right)$ increase in comfort between the initial visit and the follow-up visit 3 months later.

In contrast to the other two groups, comfort scarcely increased between dispensing (4.8) and a follow-up visit after 2 weeks (5.0) in the group with unacceptable toric fluorescein patterns with standard lens design (MC or AS). At this point the lens design was changed to a back toric lens. Because of the relatively low central difference in $k$-readings, a peripheral toric lens was chosen (two different $e$-values in two 
meridians). This resulted in an increase in comfort from the moment the lenses were introduced at the 2 -week visit to the follow-up visit at 3 months. This increase (2.3 points) was statistically significant $\left(\chi^{2}, \mathrm{p}<0.05\right)$ and resulted in a final comfort rate of 7.3 , which is equal to the average comfort scores reached with the other two lens designs.

In conclusion, there is no difference in comfort within the first 2 weeks between optimally fitted lenses and suboptimally fitted lenses. However, after 3 months there is a 2-point difference on a 10-point scale between these two groups, which is a statistically and clinically significant difference. This information is of interest to the practitioner, since it implies that even small improvements in RGP lens fits could influence comfort of wear. It should be noted that suboptimal lenses could lead to discontinuation of lens wear.

Another interesting result of this study is that corneas with lesser degrees of central corneal astigmatism can show toric fluorescein patterns because of an increase in corneal astigmatism towards the periphery and would therefore benefit from modern back toric lens designs, leading to improved comfort compared to non-toric designs.

\section{Summary}

Corneal topographers and modern, ultra sophisticated lathing techniques make it possible and easier to fit RGP lenses that follow the shape of the cornea accurately. This results in less epithelial indentation and corneal warpage, but also leads to improved comfort and might help reduce common RGP lens problems such as 3and 9-o'clock staining. A systematic approach in evaluating the RGP lens on the eye is important to maximise the technical and physiological benefits of RGP lenses. 


\section{References}

1. Dorronsoro C, Barbero S, Llorente L, Marcos S. On-eye measurement of optical performance of rigid gas permeable contact lenses based on ocular and corneal aberrometry. Optom Vis Sci 2003;80:115-25.

2. Burger R. Effect of contact lens motion on the oxygen tension distribution under the lens. Am J Optom Physiol Opt 1974;57:441.

3. Key J, 2nd. Are hard lenses superior to soft? Arguments in favor of hard lenses. Cornea 1990;9 Suppl 1:S9-11; discussion S5.

4. Chalupa E, Swarbrick HA, Holden BA, Sjostrand J. Severe corneal infections associated with contact lens wear. Ophthalmology 1987;94:17-22.

5. Nilsson SE, Montan PG. The annualized incidence of contact lens induced keratitis in Sweden and its relation to lens type and wear schedule: results of a 3-month prospective study. CLAO J 1994;20:225-30.

6. Poggio EC, Glynn RJ, Schein OD et al. The incidence of ulcerative keratitis among users of daily-wear and extended-wear soft contact lenses. N Engl J Med 1989;321:779-83.

7. Cheng KH, Leung SL, Hoekman HW et al. Incidence of contact-lens-associated microbial keratitis and its related morbidity. Lancet 1999;354:181-5.

8. Ren DH, Yamamoto K, Ladage PM et al. Adaptive effects of 30-night wear of hyper-O2 transmissible contact lenses on bacterial binding and corneal epithelium: a 1-year clinical trial. Ophthalmology 2002;109:27-39.

9. Ladage PM, Yamamoto K, Ren DH et al. Effects of rigid and soft contact lens daily wear on corneal epithelium, tear lactate dehydrogenase, and bacterial binding to exfoliated epithelial cells. Ophthalmology 2001;108:1279-88.

10. Phillips A. Rigid gas permeable and hard corneal lens fitting. In: Phillips A, Stone J, ed. Contact Lenses. London: Butterworth, 1989:333-81.

11. Douglas JP, Lowder CY, Lazorik R, Meisler DM. Giant papillary conjunctivitis associated with rigid gas permeable contact lenses. CLAO J 1988;14:143-7.

12. NAC-report. Kwantitatief onderzoek lenzenmarkt (Nederland) 2004. Hogeschool Drenthe 2004:1-57.

13. Morgan P, Efron N, Woods C et al. International Contact Lens Prescribing. Contact Lens Spectrum 2004;19(1):34-7.

14. Edwards K. Contact lens problem-solving: aspheric RGP lenses. Optician 2000;219:28-32.

15. Swarbrick HA, Hiew R, Kee AV, Peterson S, Tahhan N. Apical clearance rigid contact lenses induce corneal steepening. Optom Vis Sci 2004;81:427-35.

16. Sridharan R, Swarbrick H. Corneal response to short-term orthokeratology lens wear. Optom Vis Sci 2003;80:200-6.

17. Maeda N, Klyce SD, Hamano H. Alteration of corneal asphericity in rigid gas permeable contact lens induced warpage. CLAO J 1994;20:27-31.

18. Ruiz-Montenegro J, Mafra CH, Wilson SE, Jumper JM, Klyce SD, Mendelson EN. Corneal topographic alterations in normal contact lens wearers. Ophthalmology 1993;100:128-34.

19. Wilson SE, Lin DT, Klyce SD, Reidy JJ, Insler MS. Topographic changes in contact lensinduced corneal warpage. Ophthalmology 1990;97:734-44.

20. Wilson SE, Lin DT, Klyce SD, Reidy JJ, Insler MS. Rigid contact lens decentration: a risk factor for corneal warpage. CLAO J 1990;16:177-82.

21. Budak K, Hamed AM, Friedman NJ, Koch DD. Preoperative screening of contact lens wearers before refractive surgery. J Cataract Refract Surg 1999;25:1080-6.

22. Wang X, McCulley JP, Bowman RW, Cavanagh HD. Time to resolution of contact lensinduced corneal warpage prior to refractive surgery. CLAO J 2002;28:169-71. 
23. Lafeber R. Personal communication. 2004.

24. Carney LG, Mainstone JC, Henderson BA. Corneal topography and myopia. A cross-sectional study. Invest Ophthalmol Vis Sci 1997;38:311-20.

25. Eghbali F, Hsui EH, Eghbali K, Weissman BA. Oxygen transmissibility at various locations in hydrogel toric prism- ballasted contact lenses. Optom Vis Sci 1996;73:164-8.

26. Guillon M, Lydon DP, Wilson C. Corneal topography: a clinical model. Ophthalmic Physiol Opt 1986;6:47-56.

27. Walline JJ, Mutti DO, Jones LA et al. The contact lens and myopia progression (CLAMP) study: design and baseline data. Optom Vis Sci 2001;78:223-33.

28. Horner DG, Soni PS, Vyas N, Himebaugh NL. Longitudinal changes in corneal asphericity in myopia. Optom Vis Sci 2000;77:198-203.

29. Lindsay RG. Videokeratoscopy in contact lens practice. Cont Lens Anterior Eye 2000;23:12834.

30. Horner DG, Soni PS, Vyas N, Himebaugh NL. Longitudinal changes in corneal asphericity in myopia. Optom Vis Sci 2000;77:198-203.

31. Lindsay R, Smith G, Atchison D. Descriptors of corneal shape. Optom Vis Sci 1998;75:156-8.

32. Swarbrick HA. Mind your P's and Q's. Roger Kame award lecture. In: Global Orhtokeratology Symposium 2004; Toronto July 24. Unpublished.

33. Young G. The effect of rigid lens design on fluorescein fit. Eye Contact Lens 1998;21:41-6.

34. Kok JH, Smulders F, van Mil C. Fitting of aspheric high gas-permeable rigid contact lenses to scarred corneas. Am J Ophthalmol 1991;112:191-4.

35. Barr J. Aspheric update. Contact Lens Spectrum 1988;3(11):56-62.

36. Bennet E, et al. DW investigation of aspheric posterior Boston IV lens design. Contact Lens Forum 1987;12(4):65-9.

37. Holden T, Bahr K, Koers D, Edrington T. The effect of secondary curve lift-off on peripheral corneal desiccation. Am J Optom Physiol Opt 1987;64:313-20.

38. Jones D, Bennett E, Davis L. How to manage peripheral corneal desiccation. Contact Lens Spectrum 1989;4(5):63-6.

39. Van der Worp E, De Brabander J, Swarbrick H, Nuijts R, Hendrikse F. Corneal desiccation in rigid contact lens wear: 3- and 9-o'clock staining. Optom Vis Sci 2003;80:280-90.

40. De Brabander J, Brinkman C, Kok J, Van Mil C. Low or High Dk? Spherical or aspherical rigid gas permeable lenses? The gap between theory and practice. JBCLA 1992;15(4):169-2.

41. Ames K, Erickson p. Optimizing aspheric and spherical rigid lens perfomrance. CLAO J 1987:165-9.

42. Grosvenor T. Fitting the astigmatic patient with rigid contact lenses. In: Ruben M, Guillon M, ed. Contact lens practice. London: Chapman \& Hall, 1994:623-47.

43. Szczotka LB, Roberts C, Herderick EE, Mahmoud A. Quantitative descriptors of corneal topography that influence soft toric contact lens fitting. Cornea 2002;21:249-55.

44. Businger U, Treiber A, Flury C. The etiology and management of three and nine o'clock staining. Int Contact Lens Clin 1989;16:136-9.

45. Jones L, Jones D. Common Contact Lens Complications. Oxford: Butterworth-Heinemann, 2000:70-3.

46. Holly F. Tear film physiology in contact lens wear, Part II, Contact lens tear film interactions. Am J Optom Physiol Opt 1981;58:331-141.

47. Lowther G. Peripheral corneal staining - can it be prevented? Int Contact Lens Clin 1989;16:37.

48. Westerhout D. Toric contact lens fitting. In: Phillips A, Stone J, ed. Contact Lenses. London: Butterworth-Heinemann 1989:505-54. 
49. Carney LG. Luminance of fluorescein solutions. Am J Optom Arch Am Acad Optom 1972;49:200-4.

50. Guillon M, Sammons W. Contact lens design. In: Ruben M, Guillon M, ed. Contact Lens Practice. London: Chapman \& Hall, 1994:87-112.

51. Mountford J, Cho P, Chui WS. Is fluorescein pattern analysis a valid method of assessing the accuracy of reverse geometry lenses for orthokeratology? Clin Exp Optom 2005;88:33-8.

52. Van der Worp E, De Brabander J, Lubberman B, Marin G, Hendrikse F. Optimising RGP lens fitting in normal eyes using 3D topographic data. Cont Lens Anterior Eye 2002;25:95-9.

53. Jongsma FH, De Brabander J, Hendrikse F, Stultiens BA. Development of a wide field height eye topographer: validation on models of the anterior eye surface. Optom Vis Sci 1998;75:6977. 


\section{CHAPTER 3}

\section{Background \& Classification of Corneal Desiccation in Rigid Gas Permeable Contact Lens Wear}

"A picture tells more than a thousand words" (unknown) 


\section{Introduction}

In this chapter, 3- and 9-o'clock staining is analyzed and evaluated, focusing on the background and classification of the condition. Part 3.1 provides an overview of 3and 9-o'clock staining and its possible management options, as published in a review article in Optometry and Vision Science. Terminology, signs, symptoms, prevalence, etiology and treatment options are all discussed.

For this thesis, an image 3- and 9-o'clock staining grading scale was developed (see back cover) which is presented in part 3.2. Several grading scales to evaluate 3-and 9-o'clock staining are available, but these are all descriptive in nature. Image grading scales for corneal staining have been developed, but these are not specifically designed to grade 3- and 9-o'clock staining. The scale was developed based on available descriptive grading scales for 3- and 9-o'clock staining, general image grading scales for corneal staining and the clinical input of a large group of Dutch contact lens practitioners. 


\title{
3.1
}

\section{Corneal Desiccation in Rigid Contact Lens Wear}

\author{
3- and 9-O'Clock Staining - a review
}

Van der Worp E, de Brabander J, Swarbrick H, Nuijts R, Hendrikse F.

Optometry \& Vision Science 2003;80:280-290

Eye Research Institute Maastricht, Department of Ophthalmology Academic Hospital, Maastricht University, The Netherlands (EW, JB, RN, FH) and the School of Optometry and Vision Science, University of New South Wales, Sydney, Australia (HS) 


\begin{abstract}
The current knowledge of treatment options together with signs and symptoms, classification, prevalence and etiology of 3- and 9-o'clock staining in rigid contact lens wear are described in this review paper. In total eleven treatment options for the management of 3-and 9-o'clock staining could be found in the literature. Five of these are related to lens parameters, namely edge lift, edge thickness and shape, back surface geometry, total diameter and back optic zone radius (BOZR). Three other options are related to lens performance: movement, centration and surface wettability. Three more variables can be identified: blinking habits, tear supplements and finally switching to a hydrogel material. There is controversy on how to adjust the most important individual variables for management of 3-and 9-o'clock staining. The advocated treatment options are often related to the fitting techniques used: lid attachment or interpalpebral fit. Implications for practice and for further research are discussed.
\end{abstract}

Key words: Rigid gas permeable contact lenses, fitting techniques, 3- and 9-o'clock staining, contact lens parameters, contact lens performance 
With currently available designs and materials, the majority of contact lenses can be fitted and worn successfully. In the literature one of the most frequently reported remaining problems relates to dryness symptoms. ${ }^{1-4}$ These occur in both hydrogel and rigid gas permeable (RGP) lens wear but manifestation, etiology and severity are different. In RGP lens wear the dryness symptoms may be associated with 3- and 9-o'clock staining, also called peripheral corneal desiccation. In daily wear (DW) of RGP lenses 3- and 9-o'clock staining is very common, ${ }^{5-7}$ and has been reported in up to $80 \%$ of wearers. ${ }^{8}$ The amount of staining present varies, from transient superficial epithelial stippling to an intense staining defect with dellen formation. For contact lens practitioners management of 3- and 9-o'clock staining can be frustrating, since it is not always easy to remedy.

The objective of this paper was to search for treatment options to decrease or remedy 3- and 9-o'clock staining. Literature was searched using MEDLINE and Visionet digital databases. First, the search focused on rigid lenses in general (including hard, PMMA, gas permeable, etc). MEDLINE yielded 1048 hits for rigid lenses and alternatives, and Visionet yielded 2118 hits. Within the bibliography that was thus created, specific key words (stain, dryness, desiccation, peripheral, pannus, limbal, vascular, etc) were used to find papers related to 3- and 9-o'clock staining. In this way, 68 papers could be identified relating to 3-and 9-o'clock staining, four were retrieved from both databases, leaving 64 papers. When analyzing these papers manually based on the criterion of any involvement of treatment options for the condition, 20 papers remained. Searching for references in textbooks ${ }^{9-13}$ and internet journal databases identified another 13 papers meeting the same criteria. In this manner, ultimately 33 papers were retrieved. Since the early days of rigid lenses, there has been an interest in this subject; the oldest paper found was written by Steele in $1959 .{ }^{14}$ Peaks of interest were found in the late 1960s, late 1980s and early 1990s. The most recent papers date from the 1994-1997 period.

Finally, the remaining 33 articles were analyzed to identify if they met the criterion of randomized clinical studies into therapeutic options for 3- and 9-o'clock staining. Only five articles met this criterion. One investigated the effect of sodium hyalunorate ophthalmic solution on peripheral staining in RGP lens wearers. ${ }^{15}$ The others focused on lens design with edge lift $^{16-18}$ as the main variable, while Schnider investigated both edge lift and diameter. ${ }^{19}$ In addition, one paper by Brannen ${ }^{20}$ was found presenting a retrospective study of 700 lens wearers, with back optic zone radius (BOZR) as the main variable. In this review all relevant papers that were found in the literature will be used to create a broad overview of the topic. The papers meeting the described criteria will be highlighted. 


\section{Terminology}

Staining of the cornea in the nasal and temporal limbal areas in RGP lens wear as a result of corneal desiccation is most often referred to as peripheral corneal staining, ${ }^{18,21,22}$ peripheral corneal desiccation, ${ }^{17,23-27}$ or 3- and 9-o'clock staining. ${ }^{4,27-}$ ${ }^{29}$ Although these terms are used interchangeably, Lebow ${ }^{30}$ argues that corneal desiccation is not exactly the same as peripheral corneal staining, because corneal desiccation is only one of several factors contributing to peripheral corneal staining.

Other terms used to describe the condition are 4-8 o'clock staining ${ }^{31}$ or juxtaposition staining, ${ }^{32}$ and persistent nasal and temporal stippling. ${ }^{33}$ In more severe cases pseudopterygium, ${ }^{12,30}$ vascularized limbal keratitis, ${ }^{12,34}$ and dellen ${ }^{12,35}$ are used to describe the condition.

The term most often used to describe this complication and certainly the most striking one, is 3- and 9-o'clock staining. ${ }^{4}$ This simply describes the objective manifestation of this complication, without implying a possible cause. Therefore 3and 9-o'clock staining will be used as the standard term in this paper.

\section{Signs, symptoms and classification}

Corneal staining in the specific three and nine o'clock areas of the cornea is the most typical and most prominent sign of 3- and 9-o'clock staining, as the term suggests. Several investigators have tried to quantify the amount of staining. Apart from general grading scale systems such as the CCLRU grading scale ${ }^{36}$ and the scale designed by Efron $^{37}$ specific verbal grading scales have been designed to grade 3and 9-o'clock staining (Table 1). 6,16,18-20,30,39,41,42 One of the most applicable is the one by Schnider, ${ }^{6}$ who introduced a five point grading scale for 3- and 9-o'clock staining. Grade 0 indicates no staining, whereas grade 1 corresponds with diffuse superficial non-coalescent staining, not requiring any intervention. Grade 2 shows superficial coalescence but with no penetration of fluorescein into the deeper layers of the epithelium. Patients with this condition are advised to have regular followup checks. In grade 3 staining, a marked coalescence is seen with some penetration of fluorescein into the deeper epithelial layers. Modification of lens fit, design or wearing schedule is suggested for this stage. Grade 4 staining shows complete coalescence of staining and extensive loss of epithelial cells. In such instances Schnider's suggestion is to remove the lenses from the eye to allow epithelial regeneration, before refitting is undertaken. 


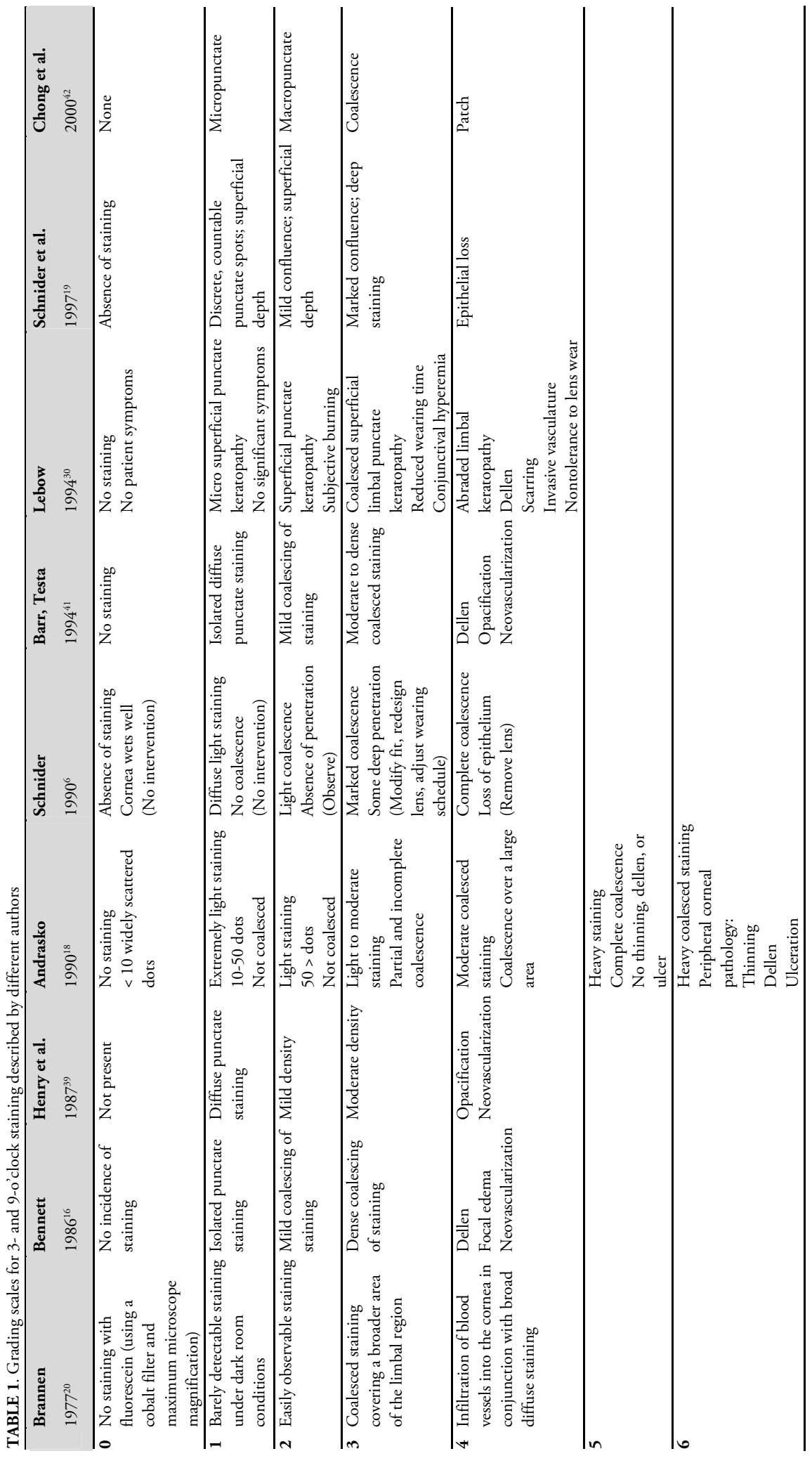


A grading system for vascularized limbic keratitis introduced by Grohe and Lebow $^{34}$ continues where the other grading scales end, thus being applicable to more severe cases. In stage 1 epithelial chafing with varying degrees of superficial punctate keratitis and heaping of hyperplastic corneal or limbal epithelium (or both) are present. Stage 2 is characterized by the occurrence of an inflammatory response. During this hyperemic phase there is increased lens awareness, bulbar conjunctival hyperemia and coalesced peripheral corneal staining. In stage 3 , conjunctival hyperemia is increased as a result of greater infiltrative reaction and expansion of the overlying corneal staining as compared to stage 2 . At this stage discomfort or pain is present and the patient's wearing time decreases. In stage 4, significant conjunctival hyperemia and staining occur, often associated with an erosion of the elevated hyperplastic epithelium. Patients are usually not able to wear their lenses any longer at this stage.

Chong et al. ${ }^{42}$ graded 3- and 9-o'clock staining in three different ways: verbal descriptors scales, photographic matching scales and continuous matching scales (using a video technique). It was suggested that the advantage of the latter technique is that the grading is non-stepwise, but a gradual continuous scale. The coefficient of repeatability of these grading systems was respectively $0.49,0.39$ and 0.49 . Based on this they concluded that all types of grading systems for 3- and 9-o'clock staining showed good reliability.

Bulbar conjunctival hyperemia is often the first subjective symptom in 3- and 9o'clock staining, ${ }^{2}$ and it is very common in the more advanced stages. ${ }^{43}$ Standard grading scales such as the CCLRU and Efron grading scales are generally used to grade bulbar hyperemia. Some investigators have recently used objective digital assessment successfully to grade bulbar hyperaemia. ${ }^{44,45}$ Chong et al. ${ }^{42}$ concluded that bulbar redness was graded with higher precision using the continuous matching scale than with photographic matching scales or verbal description scales.

Subjective symptoms of 3- and 9-o'clock staining typically include discomfort, foreign body sensation, tearing, burning, reduced wearing times and photophobia. ${ }^{4,28}$ If these symptoms occur they tend to increase during the day. ${ }^{28}$ However, subjective symptoms of ocular irritation do not necessarily correlate with objective findings. Low grades and even absence of discomfort in 3- and 9-o'clock staining have been reported frequently. ${ }^{2,15,46}$ 


\section{Prevalence}

Since the introduction of rigid corneal lenses in the early 1950s staining in the nasal and temporal limbal area has been mentioned. ${ }^{14,32,33,35}$ It has been reported that $80 \%$ of RGP wearers show some degree of 3- and 9-o'clock staining, ${ }^{8}$ but it is estimated to be of clinical significance in only $10-15 \% .{ }^{8,24}$ Andrasko $^{18}$ reported a 43 $70 \%$ prevalence, while Schnider6 observed higher than grade 1 staining in 19.8\% and severe staining in $4.3 \%$ of subjects in an extended wear (EW) study. The prevalence of 3- and 9-o'clock staining in DW seems to differ considerably from the prevalence in EW. Schnider and co-workers reported a $28 \%$ prevalence in DW and $63 \%$ in EW. ${ }^{26}$ Henry et al. ${ }^{39}$ also found a high prevalence (83\%) in EW RGP lens wear.

\section{Etiology}

A number of theories have been advanced to explain the phenomenon of 3- and 9o'clock staining. The most popular theory in the literature is the bridge effect, also called the lid gap theory (Figure. 1). Due to a bridge being formed by the upper eyelid between the edge of the lens and the cornea during blinking a gap is created under the eyelid and mucin coverage of the area under the 'bridge' may be inadequate. Lack of mucin coverage of the nasal and temporal portions of the cornea can result in poor epithelial wetting and therefore corneal staining. ${ }^{2,8,33,38,40,47}$

Decreased blinking during RGP lens wear has also often been reported as a factor in 3- and 9-o'clock staining. ${ }^{2,22,47}$ The assumption is made that discomfort will increase the influence on blinking habits. ${ }^{23}$ In particular interaction between the lens edge and the upper eyelid is thought to cause this effect. Decreased blinking results in increased tear evaporation. Large palpebral apertures have been reported also to have a negative effect for the same reason. ${ }^{29}$

Another theory, the tear meniscus theory (Figure 2), introduced by Holly ${ }^{48}$ focuses on the capillary attraction of tears in the corneal area adjacent to the lens edge which creates a tear meniscus around the lens. The thicker the lens edge and the higher the edge clearance, the greater the volume of tears attracted to this area. The tear film in the area immediately adjacent to the tear meniscus at the lens edge may therefore become very thin and can break up easily, causing a dry area and subsequent staining.

According to some authors mechanical factors play a role in the etiology of this condition. ${ }^{33,34,49,50}$ This theory states that excessive friction of the lens edge against 
the limbal area can cause 3- and 9-o'clock staining, and with-the-rule corneal toricity is recognized as a risk factor. ${ }^{12,49}$

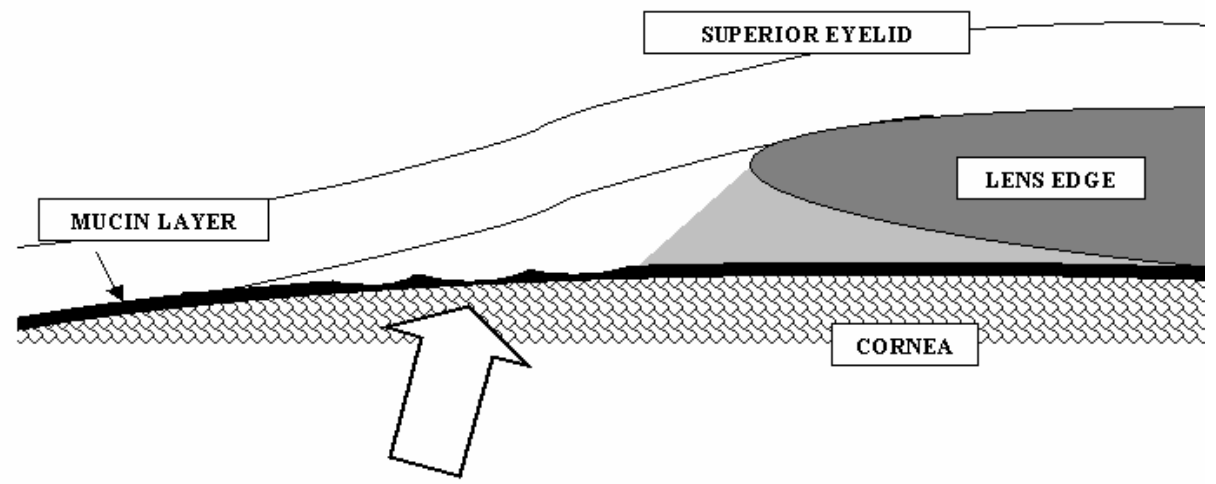

FIGURE 1. Bridge effect or lid gap theory: due to a bridge being formed by the upper eyelid between the edge of the lens and the cornea upon blinking a gap is created under the eyelid and mucin coverage (arrow) of the area under the 'bridge' may be inadequate.

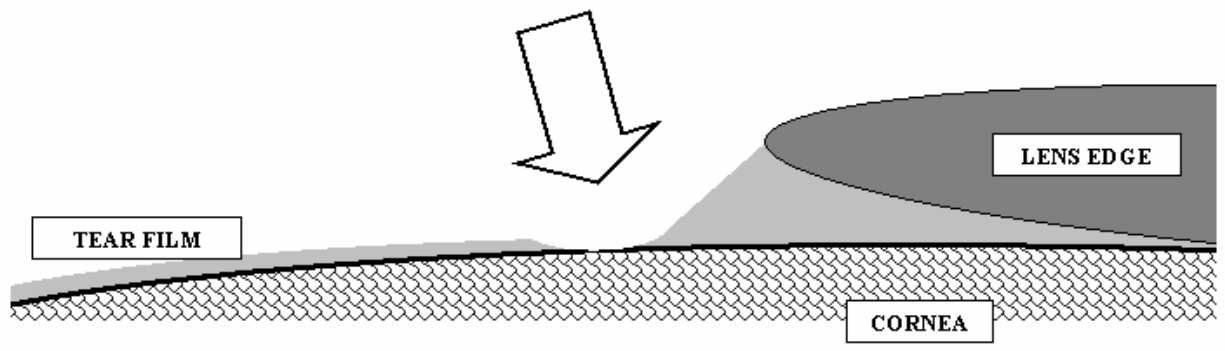

FIGURE 2. Tear meniscus theory: localized thinning (arrow) of the tear film in the area immediately adjacent to the tear meniscus at the lens edge.

Finally, there is one paper by Bell ${ }^{51}$ that suggests involvement of tear fluid dynamics in the etiology of 3- and 9-o'clock staining. Movement of a RGP lens can cause an upward and downward wake in the tear film. Within the wake, he states, swirling vortices of fluid particles may bombard the cornea and cause staining.

In summary, it can be stated that the international literature is not in agreement on the etiology of 3- and 9-o'clock staining in RGP lens wear. Different authors suggest different possibilities. The involvement of the tear film is not questioned, but is viewed from different perspectives. Based on this literature search it can be concluded that either the specific cause of 3- and 9-o'clock staining is not known or that there is no one single cause. 


\section{Treatment}

Without knowing the exact cause of a complication it is a difficult task to treat it. Strategies for decreasing 3- and 9-o'clock staining found in the literature are often based on practical experience or clinical trials where different options are tried out in an attempt to find the best solution. One paper of particular interest reports the results of a survey, with all inherent potential limitations, amongst 27 US contact lens laboratories with respect to modification of different contact lens parameters to decrease 3- and 9-o'clock staining. ${ }^{22}$ The opinion of specialists like these can be useful in creating a full picture. Another example of this is an interview by Ghormley ${ }^{24}$ with two specialists on RGP lenses, Dr. Ed Bennett and Dr. Christina Schnider, which also will be quoted often in the following text. A review paper by Jones, Bennett and Davis ${ }^{23}$ is also frequently referred to, especially because of the corneal desiccation management nomogram they presented.

Of considerable interest, due to its scientific character and its relatively recent publication, is an EW study by Schnider et al. ${ }^{19}$ of 24 patients. Based on this study they identified four factors that best represent the variables essential in 3- and 9o'clock staining management, and these will be discussed in subsequent sections of this paper.

In total eleven treatment options for the management of 3- and 9-o'clock staining could be found in the literature. Five of these are lens parameter variables: edge lift, edge thickness and shape, back surface geometry, total diameter and back optic zone radius (BOZR). Three others are related to lens performance: movement, centration and surface wettability. Two additional variables could be defined: blinking habits and tear supplements. Finally, switching to a hydrogel lens material has been mentioned as a possibility in 3- and 9-o'clock staining management. All of these options will be discussed in this overview, and are summarized in Table 2.

\section{Lens Parameters}

\section{Edge Lift}

The edge lift of a RGP contact lens can be calculated in either axial or radial directions. Both measure the height of the lens edge relative to the extension of the BOZR. However, in reality the distance from the corneal surface to the back surface of the lens edge is the clinical relevant edge clearance. This clearance is what practitioners observe and judge when evaluating fluorescein patterns, and is dependent on the rate of peripheral flattening of the cornea. 
TABLE 2. Treatment options for 3- and 9-o'clock staining

\begin{tabular}{|c|c|}
\hline Variable & Adjustment \\
\hline \multicolumn{2}{|c|}{ LENS PARAMETERS } \\
\hline Edge lift & $\begin{array}{l}\text { Is believed to be one of the key factors. Disagreement in the literature whether to re- } \\
\text { duce or increase it Both have proven to be beneficial. Increasing supports lid attach- } \\
\text { ment, leading to less influence on blinking. Decreasing reduces bridge effect and tear } \\
\text { meniscus and also encourages normal blinking. }\end{array}$ \\
\hline $\begin{array}{l}\text { Edge thick- } \\
\text { ness and } \\
\text { shape }\end{array}$ & $\begin{array}{l}\text { Both edge thickness and edge shape have an effect on bridge effect, tear meniscus and } \\
\text { blinking and should be taken into account. In higher minus lenses lenticular lenses are } \\
\text { advised. }\end{array}$ \\
\hline $\begin{array}{l}\text { Back surface } \\
\text { geometry }\end{array}$ & $\begin{array}{l}\text { Based on theoretical considerations it can be concluded that aligning the curves of the } \\
\text { lens with the cornea as much as possible (for instance by back toric and aspheric lens } \\
\text { designs) is likely to be beneficial in managing 3-and 9-o'clock staining. These issues } \\
\text { have received surprisingly little attention in the literature. }\end{array}$ \\
\hline $\begin{array}{l}\text { Lens diame- } \\
\text { ter }\end{array}$ & $\begin{array}{l}\text { There is controversy in regard to the efficacy of changing lens diameter. Larger diame- } \\
\text { ters are recommended mostly as long as thickness and optic zone diameter are reduced, } \\
\text { especially when lid attachment is desired. When adhering an interpalpebral fit philoso- } \\
\text { phy, striving for smaller diameters seems wise. }\end{array}$ \\
\hline BOZR & $\begin{array}{l}\text { Lenses fitted slightly flatter than } \mathrm{K} \text { are most commonly advised (especially if a lid at- } \\
\text { tachment fit is desired), followed by alignment fits (for a central fit). }\end{array}$ \\
\hline \multicolumn{2}{|c|}{ LENS PERFORMANCE } \\
\hline Centration & $\begin{array}{l}\text { Centration is an important factor to be considered in the management of 3-and 9- } \\
\text { o'clock staining. It is apparent that an inferior lens position should be avoided. Chang- } \\
\text { ing any of the other parameters to evaluate their effect on 3- and 9-o'clock staining } \\
\text { should not be performed without observing the effect on lens centration. }\end{array}$ \\
\hline Movement & $\begin{array}{l}\text { An adequate and smooth movement of the lens is essential to achieve adequate wetting } \\
\text { in the three and nine o'clock positions of the cornea. }\end{array}$ \\
\hline $\begin{array}{l}\text { Material } \\
\text { properties }\end{array}$ & $\begin{array}{l}\text { General agreement in the literature on contact lens material: a clean and well wetting } \\
\text { lens surface is beneficial in minimizing } 3 \text { - and 9-o'clock staining. The way to achieve } \\
\text { this is highly dependent on material characteristics and care system that is used. When } \\
\text { changing lens material, effects of lens mass on lens centration should be monitored. }\end{array}$ \\
\hline \multicolumn{2}{|c|}{ OTHER VARIABLES } \\
\hline Blinking & $\begin{array}{l}\text { It is clear that blinking habits are an essential factor. Apart from blinking exercises it is } \\
\text { advised to create a thin lens with minimal edge lift for a central fit or to increase the } \\
\text { edge lift plus diameter and flatten the BOZR to achieve lid attachment in order to } \\
\text { support proper blinking. }\end{array}$ \\
\hline $\begin{array}{l}\text { Tear sup- } \\
\text { plements }\end{array}$ & $\begin{array}{l}\text { Tear film supplementation has been shown to be helpful, although the effect is tempo- } \\
\text { rary. }\end{array}$ \\
\hline $\begin{array}{l}\text { Hydrogel } \\
\text { lenses }\end{array}$ & $\begin{array}{l}\text { Switching to a hydrogel material in chronic 3- and 9-o'clock staining is a final option. } \\
\text { Dehydration staining might become manifest, but the specific problem of 3- and 9- } \\
\text { o'clock staining will be solved. }\end{array}$ \\
\hline
\end{tabular}


Edge lift is a very important issue in 3- and 9-o'clock staining. All four studies investigating the therapeutic effect of changes in lens parameters or lens performance that met the inclusion criteria for this review use edge lift as at least one or even as the only variable. In the Jones et al. nomogram ${ }^{23}$ it was considered to be one of the four main factors while in the Buch survey it was stated to be the most influential lens parameter. In the Schnider study post lens tear film profile was considered to be the most influential factor, including both central and peripheral fluorescein fit.

Looking at the main theories on the etiology of 3- and 9-o'clock staining (bridge effect, blinking inhibition and lens edge tear meniscus) a relatively steep peripheral fit seems a logical management option. Firstly, the lack of mucin coverage of the corneal epithelium due to the bridge effect is reduced with a steeper edge. Secondly, a lower edge lift should reduce impedance of normal blinking. ${ }^{16}$ Thirdly, with lower edge clearance less tear fluid would be attracted to the lens edge tear meniscus and the adjacent area consequently could be less prone to drying. ${ }^{22,28,44}$

Some authors do agree with this argument for a reduction of edge lift, although others disagree. In the survey by Ghormley, Bennett stated that it is 'imperative' to reduce edge clearance. Out of the 26 respondents in the RGP laboratory survey, ${ }^{17}$ said they would reduce axial edge lift (versus 8 who would increase edge lift). Jones et al. ${ }^{23}$ also suggested a decrease in edge lift by using steeper peripheral curve radii and/or smaller peripheral curve widths. Holden et al. ${ }^{17}$ reached the same conclusion: they increased peripheral edge lift by $20 \mu \mathrm{m}$ steps beginning at $80 \mu \mathrm{m}$ until peripheral corneal desiccation occurred. Out of the 14 subjects who completed the study, 42\% exhibited 3- and 9-o'clock staining after the peripheral edge lift was increased to $100 \mu \mathrm{m}$. The remaining subjects showed staining at $120 \mu \mathrm{m}$ edge lift. No subject showed staining one week after being refitted with the $80 \mu \mathrm{m}$ edge lift lenses.

Lebow $^{28}$ also agreed with this finding, arguing that excessive edge clearance can generate peripheral corneal staining because of either increased lid gap or thinning of the tear film adjacent to the lens edge meniscus. However, he found a decrease in chronic 3- and 9-o'clock staining in one patient (out of 18) after changing from a low edge lift (radial edge lift $55 \mu \mathrm{m})$ to a moderate edge lift $(110 \mu \mathrm{m})$. As mentioned above, it is believed that low edge lifts could be a mechanical irritating factor to the corneal epithelium causing staining and hyperemia in the three and nine o'clock position in the limbal area. Some investigators indeed found more 3- and 9-o'clock staining with lower edge lifts. Williams ${ }^{52}$ compared axial edge lifts of 60 $\mu \mathrm{m}$ and $120 \mu \mathrm{m}$ and concluded that the lower edge lift created more peripheral staining than the higher edge lift. In another study of 18 patients fitted with 
aspheric lenses, less staining (grade 1.4 versus 2.1) was observed with the higher edge lifts compared to lower edge lifts. ${ }^{53}$ The Schnider study ${ }^{19}$ confirmed that staining levels increase with lenses that have excessively shallow or narrow lens edge tear reservoirs. Finally, Bennett ${ }^{16}$ found no difference in corneal desiccation in 14 myopic patients fitted with a conventional $(170 \mu \mathrm{m})$ edge lift on one eye and a lower $(155 \mu \mathrm{m})$ edge lift on the other eye.

Increasing the edge lift (and/or flattening BOZR and increasing lens diameter) to create a lid attachment fit has been described by Korb ${ }^{54}$ and others. ${ }^{52,53}$ With a lid attachment fit the lens moves in conjunction with the upper eyelid with every blink, thus reducing the interaction between the upper eyelid margin and the lens edge resulting in close to normal blinking habits and thereby reducing the likelihood of 3- and 9-o'clock staining. Also, lens movement with this type of fit can be improved compared to low riding interpalpebral lenses. The essential question in this approach is whether the increased edge clearance decreases 3- and 9-o'clock staining because of post lens tear film changes at the lens edge or whether the staining is due to a different dynamic fit (lens moving with the eyelid). It is difficult to provide an answer to this question based on the available literature.

In summary, lens edge clearance is believed to be one of the key factors in managing 3- and 9-o'clock staining, but it is not clear whether it is better to increase or decrease it. Flatter lenses (higher BOZR and edge lift) create lid attachment fits, which might be beneficial in decreasing 3- and 9-o'clock staining but not necessarily as a direct consequence of a change in the post lens tear film profile. On the other hand, with a decreased edge clearance, the tear film profile is directly affected, with a positive effect on 3- and 9-o'clock staining in many cases. One other aspect that should not be overlooked is that in all the studies described above it was not the actual observed edge clearance but the calculated edge lift that was investigated.

\section{Edge Thickness and Shape}

The thickness of the lens edge and/or the overall thickness of the lens has frequently been an object of study in the management of 3- and 9-o'clock staining. Applying the same theories as were applied to edge lift in the previous section, reduction of the lens edge thickness itself $5,8,29,55$ or overall lens thickness can be expected to be beneficial in managing 3- and 9-o'clock staining. ${ }^{23,24,28,48}$ An edge thickness between 90 and $120 \mu \mathrm{m}$ is advised in the interview with Schnider, which in practice means that lenses would often need some form of lenticulation. In the laboratory survey most consultants recommended reducing edge thickness by an average of $40 \mu \mathrm{m} .{ }^{22}$ Even those in favor of the lid attachment technique argued for 
a thin edge thickness to minimize bridging of the lid between the lens edge and the cornea. $^{54}$

Edge shape plays an important role in blinking behavior. La Hood states that one of the main factors determining the comfort of a RGP lens is the shape of the edge. Lenses with rounded anterior edge profiles were found to be significantly more comfortable than lenses with square anterior edges. ${ }^{56}$ Thick and uncomfortable lens edges inhibit complete blinking and decrease the frequency of blinking. ${ }^{41}$ Another reason for decreasing edge (and lens) thickness (by reducing center thickness) is the resultant decrease of lens mass and consequently improved lens centration. ${ }^{23}$ Attention must be paid to this effect especially in lenses made of highly gas permeable materials, since these lenses are often thicker by design. ${ }^{24}$

In conclusion, there is overall agreement about reducing the thickness profile and especially the lens edge thickness of a RGP lens to minimize 3- and 9-o'clock staining. Also, paying attention to edge shape seems important. Therefore, in higher minus lenses lenticular edge designs are advised to reduce edge thickness.

\section{Back Surface Geometry}

The first question that arises with regard to this topic is: what is meant by back surface geometry as a parameter variable? Basically it can be divided into two aspects. Firstly, the back surface lens geometry from the center to the periphery can be manipulated in terms of spherical or aspheric back surface geometry. Secondly, different meridians of the cornea have different curvatures and geometries. To follow the shape of the cornea as much as possible, sometimes a toric back surface design is chosen. However, the specific shape characteristics of the cornea can be much too complex for this type of design to reflect the shape of the cornea accurately. What role do these two corneal characteristics play in the management of 3and 9-o'clock staining?

Several authors ${ }^{17,23,51,57,58}$ have suggested that in theory an aspheric lens design could decrease 3- and 9-o'clock staining. First, aspheric back surface designs may follow the corneal shape more accurately, ${ }^{59,60}$ lessening areas of contact between lens and (peripheral) cornea and thus enhancing tear fluid interchange. Furthermore aspheric lens designs may be able to minimize lid-lens interaction, decreasing discomfort and interference with blinking habits.

Surprisingly, there are hardly any data available in the literature with regard to the effect of aspheric lenses versus spherical lenses on 3- and 9-o'clock staining. De Brabander et al. ${ }^{61}$ found no significant difference in 3- and 9-o'clock staining be- 
tween aspheric and spherical design lenses in two groups of 30 patients. Cheung et al. ${ }^{62}$ did not study 3- and 9-o'clock staining when looking at polynomial versus tricurve designs, but they did find a small increase in comfort (one on an eightpoint scale) for the polynomial lens design, which was not statistically significant ( $\mathrm{p}$ $>0.28$ ). Ames and Erickson ${ }^{63}$ compared spherical (bicurve) and aspheric lens types in ten patients to study comfort and centration and found the aspheric lens design to have a more central vertical position and to be more comfortable. Lens design (a simple bicurve spherical design) and manufacturing variables (different laboratories were used for different lens types) could also have contributed to this difference. Edwards ${ }^{60}$ also mentioned more central lens positioning with aspheric back surface geometries, based on manufacturers' claims. Good central lens position has proven to be beneficial in 3- and 9-o'clock staining management. In the Jones et al. nomogram, ${ }^{23}$ aspheric back surface geometry is advised to create a superiorly or centrally positioned lens and/or to create optimal edge lifts.

Concerning the other back surface geometry variable, meridional differences, we need to consider the following. In with-the-rule corneal toricity mechanical damage of the peripheral corneal epithelium by the lens may develop. A non-toric back surface geometry lens on such a cornea creates friction in the three and nine o'clock regions of the peripheral cornea and, according to Businger et al. ${ }^{49}$ a lens with a toric back surface geometry should be fitted. Jones and Jones ${ }^{12}$ also urge the use of a toric back surface design in the presence of significant corneal toricity. With toric lens designs, the corneal contour is followed much more precisely, and lid-lens interaction can be minimized. In this way, the influence on blinking habits is supposedly diminished, which can be beneficial in 3- and 9-o'clock staining management. $5,23,48$

Another reason for using back surface toric geometries on toric corneas is that this improves lens centration. Especially in with-the-rule toricity there is a tendency for the lens to ride high ${ }^{64}$ or low. ${ }^{65}$ The latter should be avoided at all times when managing 3- and 9-o'clock staining (see later).

A general rule for the degree of corneal toricity that should be fit with a toric lens is not easy to give. As a rule, 2.5 or $3.0 \mathrm{D}$ of corneal toricity or more ${ }^{64}$ is generally considered to be indicative for toric designs. However, corneal toricity may increase or decrease towards the corneal periphery thus influencing fitting characteristics. Newly developed peripheral toric back surface geometries (with one spherical and one aspheric meridian) can be used in lower degrees of corneal toricity in general, and in particular when peripheral toricity is relatively high compared to the central toricity. 
In summary, the cornea is a non-spherical surface, and different meridians show different curvatures and shapes. Based on theoretical considerations it can be concluded that aligning the curves of the lens with the cornea as much as possible (for instance by back toric and aspheric lens designs) is likely to be beneficial in managing 3-and 9-o'clock staining. These issues have received surprisingly little attention in the literature.

\section{Lens Diameter}

Although lens diameter is an important parameter, as emphasized by the Schnider study, there is disagreement in the literature on this topic. Illustrating this controversy, nine consultants in the laboratory survey would reduce lens diameter while nine others said they would increase it to manage 3- and 9-o'clock staining. ${ }^{22}$

In the discussion with Bennett, Schnider advocated large diameters: increasing lens diameter serves to better cover the problem area physically to protect it from drying, and a larger lens is often more comfortable, resulting in less inhibition of blinking. Bennett agreed with Schnider, and advised increasing lens diameter to blanket more of the corneal surface and reduce the area exposed to the air. He also stated however that this should not be done at the expense of centration, as the greater lens mass may result in an inferiorly positioned lens. In the Schnider study the extent and severity of staining decreased with well-fitted large lenses. Jones and Jones $^{12}$ also recommended large diameters in general. Furthermore, as discussed previously, large diameters are advocated to achieve a lid attachment fit, ${ }^{41}$ which can be beneficial in decreasing 3- and 9-o'clock staining.

Lebow $^{28}$ pointed out that the effect of a larger diameter is an increase in lens mass that may lead to a low lens position, which in general is discouraged. With an increase in total diameter in spherical lenses the optic zone diameter is often increased as well. Larger optic zones may increase 3- and 9-o'clock staining. ${ }^{41}$ It has been suggested that lenses with large optic zones move less, particularly laterally, due to the edge of the zone touching the flatter corneal periphery, and consequently using larger lenses with relatively small optic zones (less than $8 \mathrm{~mm}$ ) may be wise. ${ }^{21}$ Another effect of increasing the lens diameter is the effect on edge thickness. When the diameter of a minus lens is increased the edge becomes thicker. Thicker edges may decrease comfort and increase peripheral corneal staining, as mentioned above.

In summary there is controversy in regard to the efficacy of changing lens diameter. The controversy on lens diameter shows similarities with that on edge lift and lens centration. To achieve a lid attachment fit large diameters are necessary. Larger 
diameters are recommended as long as thickness (overall and edge) and optic zone diameter are not also increased. On the other hand, when using an interpalpebral fit philosophy, decreasing lens thickness, lens mass, edge thickness and optic zone diameter are desirable. With the latter goal in mind, striving for smaller diameters seems appropriate.

\section{$B O Z R$}

The question in relation to BOZR is whether it should be flatter or steeper than the flattest keratometry reading $(\mathrm{K})$ or in alignment with $\mathrm{K}$. In a 700 patient retrospective study Brannen ${ }^{20}$ found less 3- and 9-o'clock staining (11\%) in patients with a flat BOZR as compared to alignment fits $(28 \%)$ or steeply fitted lenses (34\%). A steep BOZR could impinge on the limbal sulcus during lens movement and cause epithelial damage. Grohe and Lebow ${ }^{34}$ advised alignment fits to prevent the occurrence of vascular limbal keratitis. Bennett advised fitting lenses slightly flatter than K when interviewed by Ghormley, while Schnider suggested alignment fits because both steeper and flatter than $\mathrm{K}$ lenses bear on the cornea, restrict posterior surface tear flow and disrupt the mucin layer of the cornea. For a more central lens position, alignment fits are preferred over flat fits.

Jones et al. ${ }^{23}$ advised fitting minus lenses slightly flatter than $\mathrm{K}$, in order to achieve a lid attachment fit and thus to reduce 3- and 9-o'clock staining. Williams ${ }^{52}$ reached the same conclusion: to create lid attachment fits, thinner and lightweight minus carrier lenticular designs with a flatter BOZR/cornea relationship should be used. Furthermore, most RGP laboratories in the US advised flattening the BOZR to diminish 3- and 9-o'clock staining according to the Buch study.

In summary, lenses fitted slightly flatter than $\mathrm{K}$ are most commonly advised (especially if a lid attachment fit is desired), followed by alignment fits (for a central fit). Again, the choice depends on the fitting philosophy that is followed.

\section{Lens Performance}

\section{Centration}

Contact lens centration is difficult to assess as an isolated variable because of its dependence on different parameters such as lens diameter, BOZR, edge lift, edge thickness, lens mass and back surface geometry. ${ }^{22,28,53}$ The importance of centration as such is beyond question. Jones et al. ${ }^{23}$ state that lens centration is a primary consideration in managing 3- and 9-o'clock staining. Of the four key factors identified in the Schnider study, vertical lens stability was the second most important factor while horizontal lens stability was the third most important factor influencing 3and 9-o'clock staining. 
An example of the complexity of centration in the management of 3-and 9-o'clock staining is the Jones et al. nomogram. ${ }^{23}$ Several options are given to optimize centration, including: altering BOZR (flatter for minus lenses, steeper for plus lenses or aspheric lens designs), lenticular designs (plus lenticular zones for high myopes and minus carrier edge design for low myopes and hyperopes), and decreasing center thickness.

Andrasko ${ }^{53}$ tried three different edge lifts in aspheric contact lenses and found that a more superior position achieved with high edge lift, when compared to low and medium edge lifts, led to a reduction in peripheral staining. Schnider and coworkers $^{19}$ also showed that a central or slightly superior lens position is a characteristic of lower staining levels, as opposed to low riding lenses. Henry et al ${ }^{39}$ reported that a superior-central fitting resulted in less corneal desiccation than interpalpebral fits, while inferiorly positioned lenses resulted in the greatest amount of staining. Williams $s^{52}$ also advocated thinner, lightweight, minus carrier lenticular designs with flatter BOZR/cornea relationships to create lid attachment fits and reduce 3and 9-o'clock staining. Others have also supported this approach. ${ }^{20,54}$ The importance of lens mass should not be overlooked as a factor in lens centration. Specific gravity of a material, lens diameter and center thickness directly influence lens mass. ${ }^{28}$ Increased lens mass is associated with lower lens position, which should be avoided in managing 3 - and 9-o'clock staining.

In conclusion, it can be stated that centration is an important factor to be considered in the management of 3- and 9-o'clock staining. It is apparent that an inferior lens position should be avoided. The reason why a superiorly positioned lens fit is beneficial is not clear. Possibly a lens tucked under the upper eyelid influences blinking habits less and therefore favors better wetting of the corneal epithelium. A possible negative effect of this type of lens fit that should be taken into account but that is not covered in this paper is the possible effect on corneal shape and topography. Finally, changing parameters to evaluate their effect on 3- and 9-o'clock staining should not be performed without evaluating the effect on lens centration.

\section{Movement}

Movement of the lens is not very often mentioned as a major topic in the literature, but in the Schnider study it is part of the second factor of importance. Efron ${ }^{47}$ mentions good lens movement in particular as one of the major factors in 3- and 9o'clock staining management.

In order to achieve good mucin coverage of the corneal epithelium, good movement of the lens and the resulting spreading of mucin is important. ${ }^{5}$ Mucin coverage is also dependent on microvilli distribution, which has been reported to be re- 
duced in desiccated areas. ${ }^{27}$ Andrasko ${ }^{53}$ demonstrated that lenses with steep peripheral curves tend to translate mostly vertically on the cornea during blinking. When the peripheral curves were flattened, the lens tended to translate both vertically and laterally, thus wetting the portions of the cornea adjacent to the lens edge. These findings concur with the importance of blinking as described below. Blinking and movement are obviously very closely related. Good blinking also results in a better tear flow around and under the lens, resulting in less staining. ${ }^{22,41}$ Both Bennett and Schnider confirmed this hypothesis in the Ghormley interview. Schnider advised avoiding restriction of posterior surface tear flow and disruption of the mucin layer of the cornea, and therefore advocated an alignment fit. Aspheric designs may be beneficial in achieving this type of fit because they follow the shape of the cornea more closely. Also, the discussion of lid attachment or interpalpebral fit is of interest in terms of lens movement. Lid attachment fits may provide better movement than a more stable low riding interpalpebral lens.

It can thus be stated that apart from a good (central or superior) lens position, adequate and smooth movement of the lens is essential to achieve adequate wetting of the cornea in 3- and 9-o'clock staining management.

\section{Material Properties}

The literature is reasonably united on contact lens material: a clean and wellwetting lens surface is beneficial in managing 3- and 9-o'clock staining., ${ }^{4,23,24,29,30}$ In the Schnider study it is considered to be one of the four key factors in managing 3and 9-o'clock staining, but the last in order of importance. In the Jones et al. nomogram ${ }^{23}$ optimization of material properties is one of the four key considerations when managing 3-and 9-o'clock staining.

Special attention to the cleaning regimen to ensure good wettability of the lens surface has been advocated, including the use of alcohol based cleaners. ${ }^{2,24}$ Achieving a better wettability with specific lens materials is a difficult matter, which is impossible to cover in detail here. But changing to fluoropolymer or fluorosilicone-acrylate materials has been reported to achieve better wettability and to minimize corneal staining. ${ }^{23}$ Some consultants in the survey by Buch recommended the use of a silicone-acrylate material with an average Dk of 24 , but most favored a fluorosiliconeacrylate material with an average $\mathrm{Dk}$ of 38 . The addition of fluorine monomer to silicone-acrylate formulations is believed to improve the wettability of the material. ${ }^{66}$ Others ${ }^{2,24}$ have suggested choosing lower Dk lens materials in 3- and 9o'clock staining management to improve wetting of the lens surface. 
When changing lens material, lens mass may consequently be altered, which can influence centration of the lens. This is highly dependent on individual material properties. In general, higher Dk materials may significantly increase lens mass and possibly induce inferior decentration, ${ }^{24}$ which is thought to be one of the main causes of 3- and 9-o'clock staining.

In conclusion, a clean and well wetting lens surface is beneficial in minimizing 3and 9-o'clock staining. The way to achieve this is highly dependent on material characteristics. When changing lens material, effects of lens mass on lens centration should be monitored.

\section{Other Variables}

\section{Blinking}

Blinking is one of the key variables in the management of 3- and 9-o'clock staining. ${ }^{2,22,23,47,54,67}$ Blinking is different from most of the other factors mentioned in this overview, in that it is not an isolated parameter that can easily be changed by itself. Adjustment of several lens parameters can enhance proper blinking. The assumption can be made that improved comfort will decrease the influence on blinking habits. ${ }^{23}$ In particular the interaction between the lens edge and the upper eyelid should be minimized as much as possible. This can be achieved by reducing edge lift and edge thickness. ${ }^{5}$ On the other hand, increasing edge lift, BOZR and diameter can result in a lid attachment fit. ${ }^{22,23}$ In this case, less interaction between lens edge and upper eyelid is also achieved. In addition to manipulation of lens parameters, blinking exercises have been widely advocated in managing 3- and 9o'clock staining., ${ }^{2,12,22,47,54}$

To summarize, it is clear that blinking habits are an essential factor when dealing with 3- and 9-o'clock staining. Changing blinking to a normal frequency and quality is desirable. Apart from blinking exercises, the most logical option is to create a thin lens with minimal edge lift to limit lens-lid interaction and to create good central positioning of the lens. On the other hand lid attachment fits, requiring an increase of edge lift, BOZR and diameter, may also be efficacious in optimizing blinking behavior.

\section{Tear Supplements}

The literature is almost unanimous about the tear film being the major factor implicated in 3- and 9-o'clock staining. Improvement of tear stability and volume could thus be beneficial. In the Jones et al. nomogram, ${ }^{23}$ tear film stability is one of the four key factors to be taken into consideration in 3- and 9-o'clock staining management. 
It has been suggested that tear supplements may be beneficial in managing this complication. A study by Itoi et al. ${ }^{15}$ looks into this subject, and is in fact one of the five studies found by the literature search that met all inclusion criteria. This study showed that tear supplements, both sodium hyaluronate and artificial tear supplement (containing $\mathrm{NaCl}$ and $\mathrm{KCl}$ ), brought about a significant reduction of subjective symptoms (65 and 78\% respectively) in persons suffering from 3- and 9o'clock staining. However, they found a decrease in objective signs with sodium hyaluronate only (43\%), whereas the artificial tear supplement group showed no significant change. Frequent use of tear supplements has also been mentioned by others to have a positive effect, at least temporarily, as well as punctal plugs to preserve tears. ${ }^{23,30,38,68}$

In summary, tear film supplementation has been shown to be helpful in minimizing 3-and 9-o'clock staining, although the effect is temporary.

\section{Hydrogel Lenses}

If manipulation of other variables is not able to solve the problem, a final option in chronic 3- and 9-o'clock staining is to switch to a hydrogel material, as suggested by several authors. ${ }^{2,12,23,29,47,49,69}$ This mode of lens wear is not without its own particular problems and corneal desiccation may become manifest in hydrogel lenses as dehydration staining, but the specific problem of 3- and 9-o'clock staining will be solved.

\section{Summary}

Papers about treatment options for 3- and 9-o'clock staining, especially reports of randomized clinical trials, are hard to find. Only five such papers could be distilled from the current literature. However, the prevalence of 3- and 9-o'clock staining is reported to be very high. Up to $80 \%$ of RGP wearers have been reported to show some form of 3- and 9-o'clock staining, of which $10-15 \%$ is estimated to be of clinical significance.

The available literature is not unanimous about the etiology of 3- and 9-o'clock staining. The most widely cited possible mechanism is the bridge effect, also frequently referred to as the lid gap theory, followed by the theory on blinking inhibition. Tear meniscus formation around the lens edge and mechanical factors are two other possible causes. It can be concluded that either the exact cause of 3- and 9o'clock staining is not known or that there is no one single cause. 
The objective of this paper was to search for treatment options to decrease or remedy 3-and 9-o'clock staining. In total eleven treatment options for the management of 3-and 9-o'clock staining could be found in the available literature. Five of these were related to lens parameters, three were related to lens performance and three other variables, blinking habits, tear supplements and switching to a hydrogel material, were also identified.

Edge lift (or edge clearance) is by far the most popular variable (most frequently investigated and most often referred to) of all treatment options. It seems to be one of the key factors in managing 3- and 9-o'clock staining. However, conflicting evidence is found on this topic. Both increasing and decreasing edge clearance is reported to be beneficial. It should be emphasized that by changing this parameter, not just the edge clearance is changed but a completely different fitting technique may be introduced. Higher edge lifts can result in lid attachment fits, while with lower edge lifts interpalpebral fits can be created. These fitting techniques or philosophies are directly related to other parameters that have been found to be of importance in 3- and 9-o'clock staining management, but lead to conflicting conclusions. For instance; a flatter BOZR is beneficial in creating a lid attachment fit, while alignment of the BOZR is suggested to achieve interpalpebral fits. Both are advocated in 3- and 9-o'clock staining management. Another example is lens diameter. Both decreasing (to create an interpalpebral fit), and increasing diameter (to achieve a lid attachment fit) have been reported to be beneficial.

Good blinking behavior is also considered essential, and all investigators desire blinking habits to be as normal as possible. However, again there is discussion on how to achieve this depending on the fitting philosophy used. For interpalpebral fits, thin and centrally positioned lenses are advocated in order to decrease lid-lens interaction as much as possible. For lid attachment fits large diameters and large edge lifts should be chosen to achieve a superiorly positioned lens. Blinking exercises have been suggested in general to improve blinking and to decrease 3- and 9o'clock staining. Lens centration is also pertinent to the discussion on fitting philosophies. Inferior lens position should be avoided at all times, while central or superior lens position is desirable. To achieve the latter, large lenses with a large edge clearance are desired in order to create a lid attachment fit. For interpalpebral fits the goal is a thin lens with minimal edge clearance, to achieve a centered lens position.

In the discussion on management options for 3- and 9-o'clock staining, including the fitting technique that is favored, the role of lens back surface geometry has hardly been investigated. We know that the corneal surface is non-spherical and 
furthermore that different meridians show different curvatures and shapes. Based on theoretical considerations it can be hypothesized that aligning the curves of the lens as much as possible with these specific corneal characteristics (for instance by using toric or aspheric designs) may be beneficial in managing 3- and 9-o'clock staining. Possible positive effects are a decrease of local mechanical pressure, optimizing tear flow behind the lens, improvement of centration, decrease of the bridge effect, decrease of the tear meniscus around the lens edge and minimization of lidlens interaction.

In general, unanimity is found in the literature about most of the remaining variables. Good movement is considered important in achieving proper wetting of the cornea, as are decreasing edge thickness and creating a comfortable edge shape. Well wetting lens surfaces also seem desirable in 3- and 9-o'clock staining management. In addition, tear supplements could be beneficial. If not successful with all these possibilities, changing to a hydrogel material is generally accepted as a last option to remedy this condition.

\section{Conclusions}

\section{Implications for Practice}

It is clear that solving the problem of 3-and 9-o'clock staining is not an easy task. Treatment for this condition is not straightforward, and often is not possible at all. Although there are several options found in the literature available to the practitioner, not much of this literature refers to evidence-based studies.

In total eleven variables that can be used to reduce 3- and 9-o'clock staining could be identified from the literature: edge lift, edge thickness/shape, back surface geometry, lens diameter, BOZR, centration, movement, material properties, blinking, tear supplements and hydrogel lenses. There is controversy on how to adjust individual variables, with respect to both the direction and the degree of the adjustment. In fact, this controversy concerns the most important variables: edge lift, lens diameter, BOZR, centration and blinking. On analysis of the available information, different fitting philosophies (lid attachment and interpalpebral fit) emerge as the source of most of the controversy with respect to manipulation of variables. Patients suffering from 3- and 9-o'clock staining could benefit from creating one or the other fit, although it remains unclear which one is more desirable in a particular situation. Simply switching from one technique to the other may solve the problem. In addition, other options such as increasing lens wettability (lens material and care system), use of tear supplements and blinking exercises could be helpful in managing 3 - and 9-o'clock staining. 


\section{Implications for Research}

There is still a high demand for research in this field, in order to understand the controversial issues with respect to the known variables, and thus to solve the problem of 3- and 9-o'clock staining. Most variables suggested to remedy 3- and 9o'clock staining relate to the lens fit (edge clearance, edge thickness and shape, BOZR, back surface geometry, lens diameter, centration, blinking and movement). It is hypothesized that if, with modern assessment techniques, corneal contour could be measured accurately and posterior lens surfaces could be created accordingly, controlled manipulation of the post lens tear film thickness would be possible and 3- and 9-o'clock staining could be managed better. Another key element in decreasing 3- and 9-o'clock staining may be the blinking behavior (which is clearly influenced by the lens design). It seems essential to include close monitoring of blinking patterns in any further research in this field, and to develop lenses that will minimize interference with blinking. Lens movement and centration are two other variables that could have a positive influence in management of 3- and 9o'clock staining. It would be interesting to monitor or control these variables in future research. Finally, the role of lens surface wettability should not be overlooked as a possible critical factor in the management of 3-and 9-o'clock staining. Investigation of both lens material properties and the influence of care (and cleaning) systems should be considered for future research.

\section{Acknowledgements}

This study was supported by a grant from the EC as part of BRITE-EURAM project BE 97-4608. Special thanks to Ineke Krijger of the Hogeschool van Utrecht, the Netherlands for her valuable comments. 


\section{References}

1. Brennan N, Efron N. Symptomatology of HEMA contact lens wear. Optom Vis Sci 1989;66:834-8.

2. Lowther G. Dryness, Tears, and Contact Lens Wear. Boston: Butterworth-Heinemann, 1997:84-90.

3. Guillon J-P, Young G. Subtle signs of sicca. Optician 1999;218:18-20.

4. Tomlinson A. Contact lens-induced dry eye. In: Tomlinson A, ed. Complications in Contact Lens Wear. St. Louis: Mosby, 1992:195-218.

5. Lowther G. Peripheral corneal staining - can it be prevented? Int Contact Lens Clin 1989;16:37.

6. Schnider CM. Clinical Correlations of Peripheral Corneal Desiccation. MSc thesis, University of New South Wales, 1994:10-37.

7. Jones L, Jones D. Common Contact Lens Complications. Oxford: Butterworth-Heinemann, 2000:70-3.

8. Solomon J. Causes and treatments of peripheral corneal desiccation. Contact Lens Forum 1986;11(6):30-6.

9. Ruben M, Guillon M. Contact Lens Practice. London: Chapman \& Hall, 1994.

10. Phillips A, Stone J. Contact Lenses, $3^{\text {rd }}$ ed. London: Butterworth-Heinemann, 1989.

11. Efron N. Contact Lens Complications. Oxford: Butterworth-Heinemann, 1999.

12. Jones L, Jones D. Common Contact Lens Complications. Oxford: Butterworth-Heinemann, 2000.

13. Silbert J. Anterior Segment Complications of Contact Lens Wear. New York, 1994.

14. Steele E. Observations on the fitting of corneal contact lenses. Am J Optom Arch Am Acad Optom 1959;36:194-99.

15. Itoi M, Kim O, Kimura T, Kanai A, Momose T, Kanki K, Yamaguchi T, Ueno Y, Kurokawa M, Komemushi S. Effect of sodium hyaluronate ophthalmic solution on peripheral staining of rigid contact lens wearers. CLAO J 1995;21:261-4.

16. Bennett E. The effect of varying axial edge lift on silicone/acrylate lens performance. The Contact Lens Journal 1986;14:3-7.

17. Holden T, Bahr K, Koers D, Edrington T. The effect of secondary curve lift-off on peripheral corneal desiccation. Am J Optom Physiol Opt 1987;64:313-20.

18. Andrasko G. Peripheral corneal staining: incidence and time course. Contact Lens Spectrum 1990;5(7):59.

19. Schnider C, Terry R, Holden B. Effect of lens design on peripheral corneal desiccation. J Am Optom Assoc 1997;68:163-70.

20. Brannen R. Incidence of central edema and peripheral staining as a function of contact lens fitting philosophy. J Am Optom Assoc 1977;48:391-5.

21. Lowther G. Review of rigid contact lens design and effects of design on lens fit. Int Contact Lens Clin 1988;15:378-89.

22. Buch J. Peripheral corneal staining: a survey of RGP laboratories. Contact Lens Spectrum 1997;12(5):31-7.

23. Jones D, Bennett E, Davis L. How to manage peripheral corneal desiccation. Contact Lens Spectrum 1989;4(5):63-6.

24. Ghormley N, Bennet E, Schnider C. Corneal desiccation-clinical management. Int Contact Lens Clin 1990;17:5-8.

25. Dougal J. Abrasions secondary to contact lens wear. In: Tomlinson A, ed. Complications in Contact Lens Wear. St. Louis: Mosby, 1992:123-56. 
26. Schnider C, Terry R, Holden B. Effect of patient and lens performance characteristics on peripheral corneal desiccation. J Am Optom Assoc 1996;67:144-50.

27. Bell G. A new theory on 3- and 9-o'clock staining. Contact Lens Spectrum 1997;12(12):44-7.

28. Lebow K. Reduce three-and-nine corneal staining with moderate edge lift profiles. Contact Lens Spectrum 1990;5(7):20-33.

29. Gasson A, Morris J. Contact Lens Manual. London: Butterworth-Heinemann, 1998:318-21.

30. Lebow K. Peripheral corneal staining. In: Silbert J, ed. Anterior Segment Complications of Contact Lens Wear. New York: Churchill Livingstone, 1994:59-90.

31. Arner R. Corneal contact lens design by minimal corneal insult. J Am Optom Assoc 1969;40:308-9.

32. Barabas R, Fontana A. Juxtaposition staining. Contacto 1967;11:3-6.

33. Graham R. Persistent nasal and temporal stippling. Contacto 1968;12:20-1.

34. Grohe R, Lebow K. Vascularized limbal keratitis. Int Contact Lens Clin 1989;16:197-209.

35. Mackie I. Lesions at the corneal limbus at 3 o'clock and 9 o'clock in association with the wearing of corneal lenses. In: Dabezies O, Laues H, A S, GP H, eds. Contact Lenses. New York: S. Karger, 1967:66-73.

36. Terry R, Schnider C, Holden B, Cornish R, Grant T, Sweeney D, La Hood D, Back A. CCLRU standards for success of daily and extended wear contact lenses. Optom Vis Sci 1993;70:234-43.

37. Efron N. Contact Lens Complications. Oxford: Butterworth-Heinemann, 1999:161-89.

38. Bennett E, Egan D. Rigid gas permeable lens problem solving. J Am Optom Assoc 1986;57:504-11.

39. Henry V, Bennett S, Forrest J. Clinical investigation of the Paraperm EW rigid gas permeable contact lens. Am J Optom Physiol Opt 1987;64:313-20.

40. Schnider C. Rigid gas permeable extended wear. Contact Lens Spectrum 1990;5(9):101-6.

41. Barr J, Testa L. Corneal epithelium 3 and 9 o'clock staining studied with the specular microscope. Int Contact Lens Clin 1994;21:105-11.

42. Chong T, Simpson T, Fonn D. The repeatability of discrete and continuous anterior segment grading scales. Optom Vis Sci 2000;77:244-51.

43. Snyder C. Dry eye symptoms and contact lens wear. In: Silbert J, ed. Anterior Segment Complications of Contact Lens Wear. New York: Churchill Livingstone, 1994:213-20.

44. Guillon M, Shah D. Objective measurement of contact lens-induced conjunctival redness. Optom Vis Sci 1996;73:595-605.

45. Papas E. Key factors in the subjective and objective assessment of conjunctival erythema. Invest Ophthalmol Vis Sci 2000;41:687-91.

46. Guillon J, Guillon M. The role of tears in contact lens performance and its measurement. In: Ruben M, Guillon M, eds. Contact Lens Practice. London: Chapman \& Hall, 1994:453-83.

47. Efron N. Contact Lens Complications. Oxford: Butterworth-Heinemann, 1999:75-81.

48. Holly F. Tear film physiology in contact lens wear, Part II, Contact lens tear film interactions. Am J Optom Physiol Opt 1981;58:331-41.

49. Businger U, Treiber A, Flury C. The etiology and management of three and nine o'clock staining. Int Contact Lens Clin 1989;16:136-40.

50. Cotie B. How to manage 3- and 9-o'clock staining. Contact Lens Forum 1990;15(5):42-3.

51. Bell G. How's your glide? A sensible approach for rating RGP movement. Rev Optom 1996;133(3):57-61.

52. Williams CE. New design concepts for permeable rigid contact lenses. J Am Optom Assoc 1979;50:331-6.

53. Andrasko G. Peripheral corneal staining: Edge lift \& extended wear. Contact Lens Spectrum 1990;5(8):33-5. 
54. Korb D, Korb J. A new concept in contact lens design; parts 1 \& 2. J Am Optom Assoc 1970;41:1-12.

55. Efron N. Blinking. In: Efron N, ed. Contact Lens Complications. Oxford: ButterworthHeinemann, 1999:3-9.

56. La Hood D. Edge shape and comfort of rigid lenses. Am J Optom Physiol Opt 1988;65:613-8.

57. Bennett E. DW investigation of aspheric posterior Boston IV lens design. Contact Lens Forum 1987;12(4):65-9.

58. Barr J. Aspheric update. Contact Lens Spectrum 1988;3(11):56-62.

59. Kok J. New developments in the field of contact lenses. PhD thesis, University of Amsterdam, 1991:17-25.

60. Edwards K. Contact lens problem-solving: aspheric RGP lenses. Optician 2000;219:28-32.

61. de Brabander J, Brinkman C, Kok J, van Mil C. Low or high Dk? Spherical or aspherical rigid gas permeable lenses? The gap between theory and practice. J BCLA 1992;15:169-2.

62. Cheung S, Cho P, Douthwaite W. Clinical performance of polynomial lenses versus tri-curve lenses II: ocular response. Contact Lens Ant Eye 2001;24:108-14.

63. Ames K, Erickson P. Optimizing aspheric and spherical rigid lens performance. CLAO J 1987;13:165-69.

64. Grosvenor T. Fitting the astigmatic patient with rigid contact lenses. In: Ruben M, Guillon M, eds. Contact Lens Practice. London: Chapman \& Hall, 1994:623-47.

65. Westerhout D. Toric contact lens fitting. In: Phillips A, Stone J, ed. Contact Lenses, $3^{\text {rd }}$ ed. London: Butterworth-Heinemann, 1989:505-54.

66. Doane, M. An instrument for in vivo tear film interferometry. Optom Vis Sci 1989;66:383-8.

67. Sarver M, Nelson J, Polse K. Peripheral corneal staining accompanying contact lens wear. J Am Optom Assoc 1969;310-3.

68. Itoh R, Yokoi N, Kinoshita S. Tear film instability induced by rigid contact lenses. Cornea 1999; 18:440-3.

69. Brennan N, Bruce A. A Guide to Clinical Contact Lens Management. Bulach/ Zurich: CIBA Vision, 1993:52. 


\section{2}

\section{The Development of an Image Grading Scale with High Linearity for 3- and 9-O'Clock Staining}

Van der Worp E, de Brabander J, Swarbrick H, Hendrikse F.

Published in part in Optometry \& Vision Science 2008;85(9):857-66.

Eye Research Institute Maastricht, Department of Ophthalmology Academic Hospital, Maastricht University, The Netherlands (EW, JB, FH), School of Life and Health Sciences, Aston University, Birmingham, United Kingdom (JW) and the School of Optometry and Vision Science, University of New South Wales, Sydney, Australia (HS) 


\begin{abstract}
Purpose: The condition of 3- and 9-o'clock staining is a common rigid gas permeable lens complication. Currently, no specifically designed image based grading scale is available to clinicians to quantify this condition. The aim of this study was to develop an image grading scale for 3- and 9-o'clock staining with high linearity.
\end{abstract}

Method: A five-point image grading scale for 3- and 9-o'clock staining was developed based on currently available descriptive scales for 3-and 9-o'clock staining and available general image grading scales for corneal staining. A combination of a background photograph and an overlaid pictorial representation was used to create the desired images. The input of 182 contact lens practitioners in the Netherlands, divided into 51 teams, was used to further refine the scale and the resulting scale was objectively tested for linearity using previously validated image analysis.

Results: The image representing grade 2.03 - and 9-o'clock staining was assessed by 14 teams $(27 \%)$ as grade 2.0 whereas 32 teams $(58 \%)$ graded the image as 2.5 , while grade 3.0 was reported four times $(8 \%)$ and once (2\%) grade 1.5 was scored. This clinical opinion was used in addition to the currently available scales and input of the researchers at the University of Maastricht to further refine the scale. The resulting scale showed a linear increase between grades units for area of detected edges $\left(\mathrm{r}^{2}=0.99\right)$. The intensity of the green coloration increased linear as well $\left(r^{2}=0.96\right)$, but the relative green coloration was less linear due to an increase in red intensity in the grade 4.0 image $\left(r^{2}=0.70\right)$. The number of affected areas (particles) increased initially, but decreased with higher severity of the condition, $\left(r^{2}=0.02\right)$, indicating coalescence of staining areas.

Conclusion: The 3- and 9-o'clock staining image grading scale that was developed and that is presented in this study proved to be linear in nature and therefore it is considered appropriate for practitioners to interpolate between scale images and use small incremental steps to increase discrimination when grading this condition.

Key words: Grading scale, rigid gas permeable contact lenses, 3- and 9-o'clock corneal staining 
Epithelial staining in the three and nine o'clock areas of the cornea is a well known condition in rigid gas permeable lens wear. However, reported incidence rates vary greatly, ranging from $25 \%$ to $90 \% .^{1-4}$ One of the reasons for this large variation may be that there is no uniform way of classifying the condition. The clinical presentation of 3- and 9-o'clock staining can vary from transient superficial epithelial stippling to deep corneal defects (with stromal involvement) that stain intensely.

The clinical relevance of grading corneal staining is, apart from discomfort and possibly corneal scarring, the potential invasion of the cornea by pathogenic microorganisms via damaged epithelial cells and the risk of the development of a corneal infection. ${ }^{5,6}$

Image grading scales for corneal staining are available to practitioners, ${ }^{7-10}$ but these are not specifically designed to grade 3- and 9-o'clock staining. Several specific grading scales to evaluate 3- and 9-o'clock staining have been developed or are used for clinical studies (see table 1-part 3.1), but these are all descriptive in nature. ${ }^{2,11-18}$ Most of these grading scales use five units to describe the severity of the condition, where generally grade 0 indicates no corneal staining whereas grade 1.0 corresponds with diffuse superficial non-coalescent staining. In grade 2.0 superficial coalescence is noted, but without penetration of fluorescein into the deeper layers of the epithelium and in grade 3.0 a marked coalescence is seen with some penetration of fluorescein into the deeper epithelial layers. Grade 4.0 staining shows complete coalescence and/or extensive loss of epithelial cells with possibly stromal involvement. The affected area in 3- and 9-o'clock staining is usually limited to small temporal and nasal portions of the cornea - e.g. area 2 and 3 of the cornea classification system (Figure. 1.) of the CCLRU. ${ }^{10}$

Whichever grading scale is used, there is a large inter- and intra-observer variability. ${ }^{19,20}$ Interpolating between grading images (such as to one tenth of a unit) instead of using whole units can be helpful in increasing discrimination. ${ }^{21}$ However, this is dependent upon a linear incremental increase in severity between grades. Without a linear relationship, half or 0.1 step grading increments will not correspond to the exact grade the practitioner intends to assign. ${ }^{21}$

The purpose of this study was to develop an image based grading scale for 3- and 9-o'clock staining that is linear in nature and which can be of value to practitioners to evaluate and follow the condition in clinical practice. 


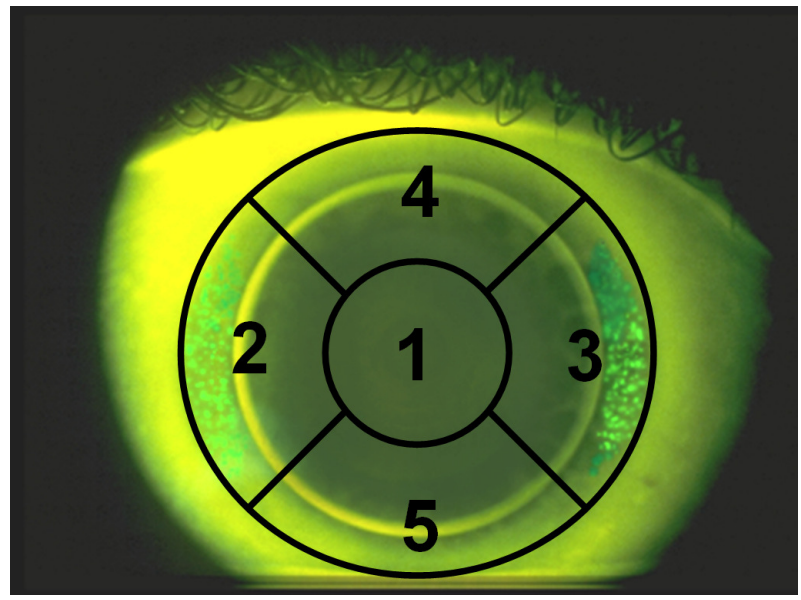

FIGURE 1. The affected area in 3and 9-o'clock staining is limited to area 2 and 3 of the CCLRU classification system

\section{Methods}

It was decided to use a five point unit scale as the basis for the 3-and 9-o'clock staining image grading scale, in line with almost all currently available descriptive grading scales for this condition ${ }^{22}$ and image grading scales for general corneal staining. ${ }^{7-10}$ Based on the existing scales, a baseline scale to grade 3- and 9-o'clock staining was developed. A combination of a background photograph and an overlaid pictorial representation of staining was used: a photograph of an eye wearing a rigid gas permeable lens was chosen to reflect an actual clinical situation and the amount of staining was manually modified with photo editing software (Photoimpressions ${ }^{\circ}$, version $5, \mathrm{ArcSoft}^{\circ}$ ) to match the desired grade of staining as subjectively identified by the investigators.

In addition to this, an input of a large group of contact lens practitioners was requested to aid in the development process of the scale. An image representing grade 2.0 staining on the baseline scale was presented to 182 contact lens practitioners (contact lens specialists and optometrists) at six different locations throughout the Netherlands. The practitioners were divided into 51 teams (with 3.6 persons on average per team). These teams of clinicians were asked to grade the 3- and 9o'clock staining image on a $0-4$ scale, choosing between four options only: grade 1.5 , grade 2.0 , grade 2.5 or grade 3.0 . The outcome of this clinical evaluation together with the input of the investigators at the University of Maastricht was used to further refine the baseline scale.

In order to test the linearity of the resulting scale after adjustments, a computer software program (LabView and Vision Software, National Instruments, Austin 
TX, USA) was used to objectively quantify the Tag Image File Format (TIFF) images. These images were analysed three times nasally and temporally for each of the five grading scales units. The images were evaluated using previously validated image analysis techniques, ${ }^{23}$ involving edge detection, color extraction, and count of affected areas (particles). Edge detection is used to get an idea about the size of the area that is affected in different stages of the condition. To identify the edges of the affected area, each pixel is compared to its neighbors on $3 \times 3$ grids. The intensity of the green color plane was evaluated as a percentage of the maximum light intensity possible and the intensity of the green was compared to the combined intensity of green, red and blue to calculate the relative green coloration (expressed as a percentage of the total). In addition to the above techniques, the total number of discrete edge detected areas of staining (e.g. the number of affected areas, called particles) was counted in the specified regions to evaluate whether an increase in the number of staining areas was present.

\section{Results}

When the grade 2.0 image of the developed baseline grading scale was presented to 51 teams of practitioners, 14 teams $(27 \%)$ assessed this image as grade 2.0, 32 teams $(58 \%)$ graded the image as 2.5 , while grade 3.0 was reported four times $(8 \%)$ and once (2\%) grade 1.5 was scored (Figure 2). At three different locations around the country grade 2.0 and grade 2.5 were reported equally frequent, while at the three other locations grade 2.5 was reported most frequent. The mean score of all teams combined was $2.4 \pm \mathrm{SD} 0.3$. This clinical assessment was used in addition to the opinion of the investigators at the University of Maastricht to further refine the 3- and 9-o'clock grading scale. The resulting five point 3- and 9-o'clock staining image grading scale is presented in Figure 3 and in full color on the back cover of this thesis.

The resulting scale was evaluated for linearity using objective image analysis: edge detection, green intensity/relative green coloration and number of affected areas (Figure 4.). The area of detected edges increased gradually from $1.5 \%$ in grade 0 to $43.8 \%$ in grade 4.0 , and a high linearity was found for this variable $\left(r^{2}=0.99\right)$. The green intensity increased linearly from $55.5 \%$ to $63.8 \%$ with increasing scale grade units $\left(r^{2}=0.96\right)$, but the relative green coloration was less strongly correlated with increasing scale grade units due to an increase in red intensity in the grade $4.0 \mathrm{im}$ age $\left(r^{2}=0.70\right)$. The number of affected areas (particles) increased initially in the lower grade units, but decreased in the higher grades $\left(r^{2}=0.02\right)$, indicating coalescence of the staining areas (Figure 5). 


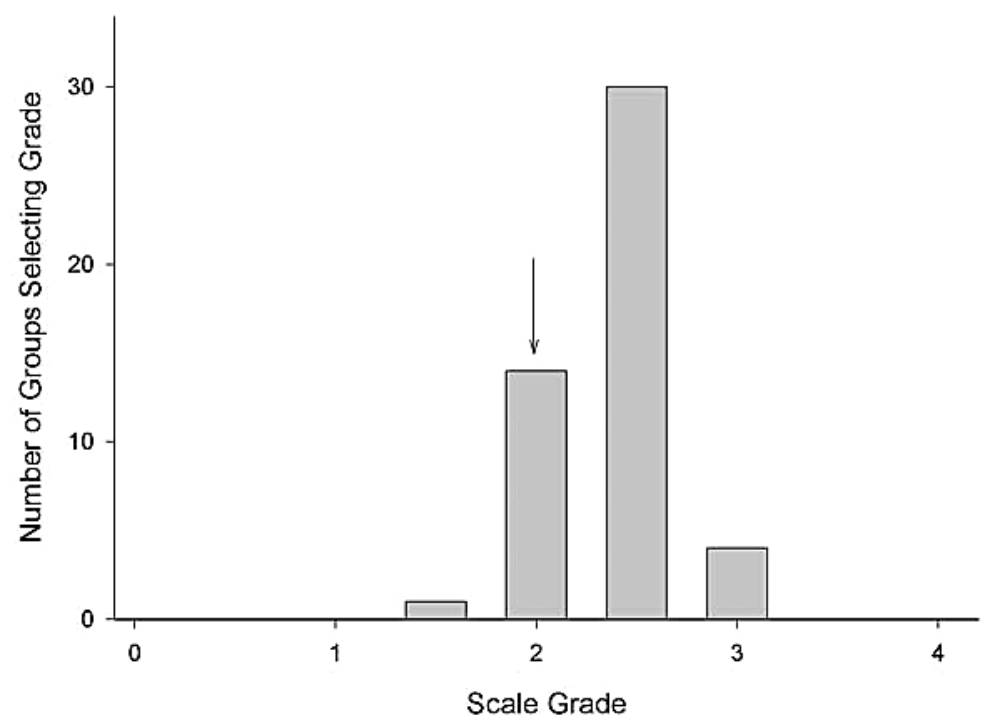

FIGURE 2. Scores of all groups of practitioners when assessing the image representing grade 2.0 .

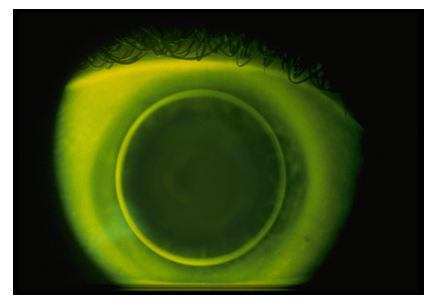

Grade 0

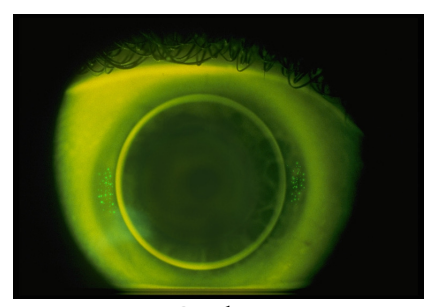

Grade 1

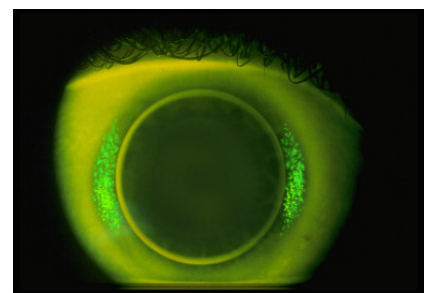

Grade 3

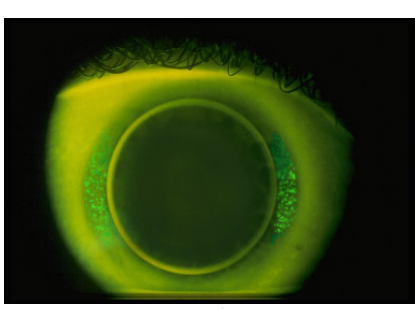

Grade 2

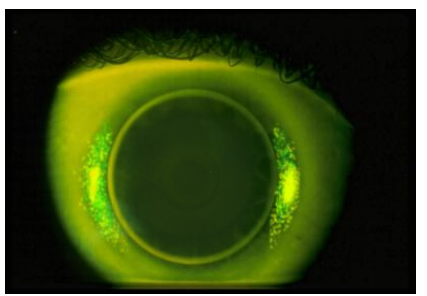

Grade 4

FIGURE 3. Units 0-4 of the resulting image 3- and 9-o'clock grading scale. 


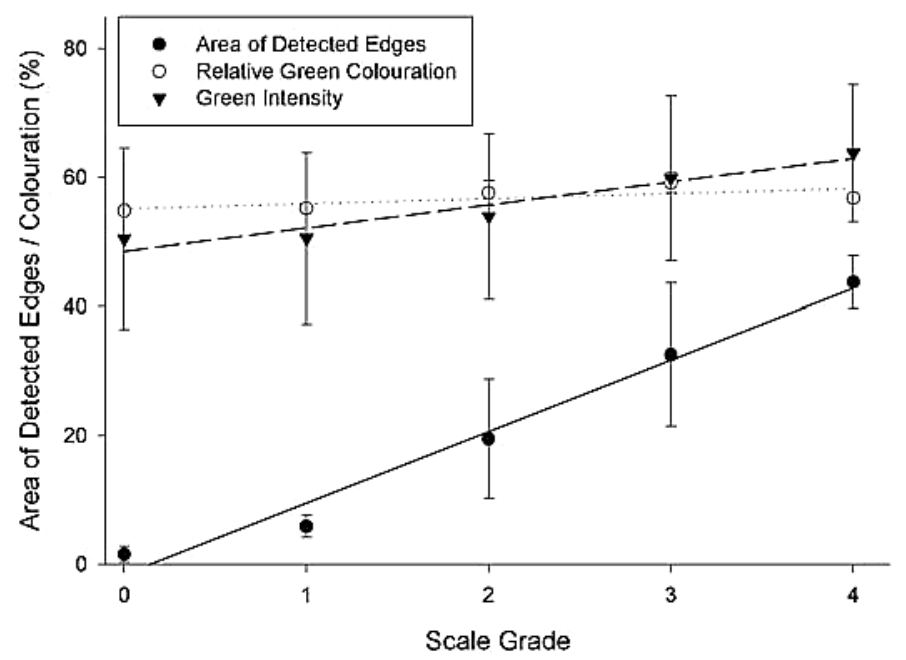

FIGURE 4. Percentage of area of detected edges, green intensity and relative green coloration for all five grading scale units.

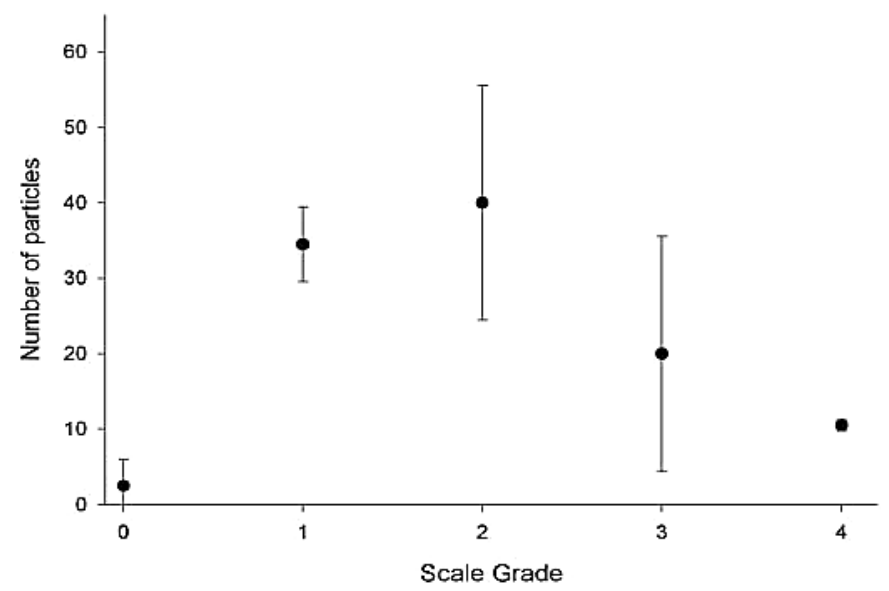

FIGURE 5. Number of particles detected for all five grading scale units. 


\section{Discussion}

Different types of grading scales are available: descriptive (written) scales and two types of image grading scales: photographic scales (photos of different stages of the condition) and pictorial scales (created by artists). Chong et al. ${ }^{18}$ compared image (photographic) grading scales with descriptive scales and found a better repeatability for the image scale with regards to the grading of corneal staining. Efron et al. ${ }^{24}$ compared two pictorial sets of grading scales $\left(\mathrm{Efron}^{7}\right.$ and Annunziato ${ }^{8}$ ) with two photographic scales (Vistakon ${ }^{9}$ and the CCLRU ${ }^{10}$ ). They concluded that the use of the pictorial grading scales generally results in lower grading estimates and better grading reliability than the use of photographic grading scales. With all four scales, practitioners showed a $95 \%$ confidence limit of $+/-1.2$ grading scale units (range $+/-0.7$ to $+/-2.5$ grading scale units). This indicates according to the authors, that a change or difference in severity of more than 1.2 grading scale units can be considered to be clinically relevant.

The major advantage of photographic images over pictorial scales is that they represent a more realistic clinical picture. However, when developing photographic scales it is a challenge to find a picture that represents exactly the desired grade. Furthermore, differences in anatomy between eyes, lighting conditions and other observational variables hamper the clinical assessment and interpretation of the photographic grades. All these disadvantages are overcome by pictorial scales in which the condition of interest is sketched at different levels of severity. This option obviously lacks a level of realism, which might be a disadvantage when used in a clinical setting. For the development of this 3- and 9-o'clock staining scale, a combination of a background photograph and an overlaid pictorial representation of staining was used to benefit from the advantages both systems have to offer.

One other difficulty that arises with regard to grading corneal staining is that the surface area affected might not be in line with the intensity of the staining. All the descriptive scales and most of the image scales combine these characteristics, but the Vistakon grading scale ${ }^{9}$ assesses depth and type of staining separately whereas the CCLRU grading scale differentiates depth, type and also extent of staining. ${ }^{10}$ Although in general this may be a more accurate method in general to describe the condition, grading two or three different aspects of staining is more time consuming, which is a disadvantage in clinical practice. In 3- and 9-o'clock staining, the variation in the extent of corneal staining is limited to small portions of the nasal and temporal cornea, and it seems therefore less relevant to grade extent and intensity separately in this condition. Therefore it was decided to use only one variable to grade corneal staining. To acknowledge any potential variance in staining inten- 
sity versus the extent of the staining, slight differences between intensity and extent of the staining (deeper and smaller versus larger and more superficial) are marked nasally and temporally in the developed scale.

In the opinion of the Dutch practitioners who observed the proposed grade 2.0 image of the scale, the severity of the 3- and 9-o'clock staining was somewhat underrated, with a mean score of grade 2.4 . The difference between the practitioners' outcome and the intended score is relatively small and falls easily within what has been shown to be 'the variability of clinical grading' (a change or difference in severity of 1.2 grading scale units or over is considered clinically significant ${ }^{24}$ ). Nevertheless, for the development of this new scale the outcome was used to refine the baseline scale: the amount of staining in the grade 2.0 image was slightly reduced and consequently the images of grades 1.0, 3.0 and 4.0 also had to be adjusted to stay in line with the grade 2.0 image.

The next step in the development of the grading scale was to evaluate the linearity of the resulting scale. To allow the use of interpolations between grading images (such as one tenth of a unit) which increases discrimination, there should be a linear incremental increase in severity between grading scale units. ${ }^{21}$ For comparison, the linearity for the Efron grading scale using edge detection was $\mathrm{r}^{2}=0.49$ while it was $\mathrm{r}^{2}=0.51$ for the CCLRU scale. For the developed 3- and 9-o'clock staining grading this value was found to be $r^{2}=0.99$. For relative coloration of corneal staining, the linearity of the Efron grading scale achieved $r^{2}=0.55$ and $r^{2}=0.86$ for the CCLRU scale, whereas it was $\mathrm{r}^{2}=0.70$ for the 3 - and 9-o'clock staining scale. The reason for the lower linearity with relative coloration than with edge detection is thought to be an increase in red intensity in the grade 4.0 image. But in general the linear correlation between units for the developed 3- and 9-o'clock staining grading scale was found to be high, with only between $2 \%$ (edge detection) and 30\% (relative coloration) of the variance not accounted for. In addition, green intensity also showed a very high linearity $\left(\mathrm{r}^{2}=0.96\right)$, but no corresponding data are available for the Efron and CCLRU scales for comparison. In summary, the developed 3- and 9-o'clock staining grading scale proved to be linear in nature and therefore it is appropriate to increment between grades images with this scale and parametric statistics can be applied when comparing clinical trial results. The number of particles detected showed a decrease with higher levels of severity of 3- and 9-o'clock staining due to coalescence of corneal staining, as expected from previous descriptive scales. ${ }^{2,11-18}$ 


\section{Conclusions}

In conclusion, there is no image-based 3- and 9-o'clock staining grading scale available for practitioners despite the high incidence of this complication in rigid gas permeable lens wear. The 3- and 9-o'clock staining image grading scale that was developed and is presented in this study proved to be linear in nature and therefore it is considered appropriate for practitioners to interpolate between scale images and use small incremental steps to increase discrimination when grading this condition. 


\section{References}

1. Lowther GE. Dryness, Tears, and Contact Lens Wear: Clinical Practice in Contact Lenses. Boston: Butterworth-Heinemann, 1997:84-90.

2. Schnider C. Clinical Correlations of Peripheral Corneal Desiccation. MSc thesis. Sydney: University of New South Wales; 1994.

3. Jones L, Jones D. Common Contact Lens Complications. Oxford: Butterworth-Heinemann, 2000:70-3.

4. Solomon J. Causes and treatments of peripheral corneal desiccation. Contact Lens Forum 1986;11:30-6.

5. Fletcher EL, Fleiszig SM, Brennan NA. Lipopolysaccharide in adherence of Pseudomonas aeruginosa to the cornea and contact lenses. Invest Ophthalmol Vis Sci 1993;34:1930-6.

6. Klotz SA, Au YK, Misra RP. A partial-thickness epithelial defect increases the adherence of Pseudomonas aeruginosa to the cornea. Invest Ophthalmol Vis Sci 1989;30:1069-74.

7. Efron N. Grading scales for contact lens complications. Ophthalmic Physiol Opt 1998;18:182-6.

8. Annunziato R, Davidson R, Christensen MT et al. Atlas of Slit lamp Findings and Contact LensRelated Anomalies. Forth Worth: Southwest Independent Institutional Review Board, 1992.

9. Andersen JS, Davies IP, Kruse A, Løfstrom T, Ringmann LA. Handbook of Contact Lens Management. Jacksonville: Vistakon, 1996.

10. Terry RL, Schnider CM, Holden BA et al. CCLRU standards for success of daily and extended wear contact lenses. Optom Vis Sci 1993;70:234-43.

11. Brannen R. Incidence of central edema and peripheral staining as a function of contact lens fitting philosophy. J Am Optom Assoc 1977;48:391-5.

12. Bennet $\mathrm{E}$. The effect of varying axial edge lift on silicone/acrylate lens performance. Contact Lens Journal 1986;14:3-7.

13. Henry V, Bennett S, Forrest J. Clinical investigation of the Paraperm EW rigid gas permeable contact lens. Am J Optom Physiol Opt 1987;64:313.

14. Andrasko G. Peripheral corneal staining: incidence and time course. Contact Lens Spectrum 1990;5(7):59.

15. Barr J, Testa L. Corneal epithelium 3 and 9 o'clock staining studied with the specular microscope. Int Contact Lens Clin 1994;21:105-11.

16. Lebow K. Peripheral corneal staining. In: Silbert J, ed. Anterior Segment Complications of Contact Lens Wear. Edinburgh: Churchill Livingstone, 1994:59-90.

17. Schnider CM, Terry RL, Holden BA. Effect of lens design on peripheral corneal desiccation. J Am Optom Assoc 1997;68:163-70.

18. Chong T, Simpson T, Fonn D. The repeatability of discrete and continuous anterior segment grading scales. Optom Vis Sci 2000;77:244-51.

19. Efron N, Morgan P, Jagpal R. The combined influence of knowledge, training and experience when grading contact lens complications. Ophthalmic Physiol Opt 2003;23:79-85.

20. Fieguth P, Simpson T. Automated measurement of bulbar redness. Invest Ophthalmol Vis Sci 2002;43:340-7.

21. Wolffsohn J. Incremental nature of anterior eye grading scales determined by objective image analysis. Br J Ophthalmol 2004;88:1434-8.

22. Van der Worp E, De Brabander J, Swarbrick H, Nuijts R, Hendrikse F. Corneal desiccation in rigid contact lens wear: 3- and 9-o'clock staining. Optom Vis Sci 2003;80:280-90.

23. Wolffsohn J, Purslow C. Clinical monitoring of ocular physiology using digital image analysis. Contact Lens Ant Eye 2003;26:27-35.

24. Efron N, Morgan P, Smaragda K. Validation of grading scales for contact lens complications. Ophthal Physiol Opt 2001;21:17-9.Chapter 4 



\section{CHAPTER 4}

Clinical Studies: Evaluation of

3- and 9-O'Clock Staining in

Existing Rigid Gas Permeable

Contact Lens Wearers

"An adventure is only an inconvenience rightly considered" (G.K. Chesterton) 


\section{Introduction}

Chapter 4 of this thesis presents the results of clinical studies evaluating 3- and 9o'clock staining in existing rigid gas permeable lens wearers, in order to better understand the condition and its etiology. The relationship between the signs and the symptoms accompanying 3- and 9-o'clock staining (bulbar hyperemia, conjunctival staining and lens comfort) is investigated in part 4.1, while part 4.2 evaluates contact lens related variables in existing lens wearers that could potentially contribute to the development of the condition. It tries to determine risk factors regarding 3- and 9-o'clock staining. 


\section{1 \\ Evaluation of Signs and Symptoms in 3- and 9-O'Clock Staining}

Van der Worp E, de Brabander J, Swarbrick H, Hendrikse F.

Optometry \& Vision Science - Accepted

Eye Research Institute Maastricht, Department of Ophthalmology Academic Hospital, Maastricht University, The Netherlands (EW, JB, FH) and the School of Optometry and Vision Science, University of New South Wales, Sydney, Australia (HS) 


\begin{abstract}
Purpose. This study evaluates the relationship between bulbar hyperemia, conjunctival staining, lens comfort and 3- and 9-o'clock corneal staining in rigid gas permeable (GP) contact lens wear.
\end{abstract}

Methods. The study involved 55 GP contact lens wearers (with and without 3and 9-o'clock staining) and 26 non-lens wearers. Corneal staining was graded based on a specially designed image grading scale developed at the University of Maastricht. The Efron grading scale was used to grade bulbar hyperemia and conjunctival staining. Ocular comfort and lens comfort were analyzed using a visual analogue scale (VAS) and a questionnaire to differentiate symptomatic from asymptomatic subjects.

Results. Bulbar hyperemia showed a significant but low correlation with corneal staining, and eyes with conjunctival staining showed more corneal staining than eyes without conjunctival staining. No correlation was found between staining in the nasal and temporal regions of the cornea in subjects with substantial 3- and 9o'clock staining. No correlation was found between VAS comfort scores and corneal staining. In those subjects with substantial 3- and 9-o'clock staining, symptomatic subjects showed more corneal staining than asymptomatic subjects.

Conclusions. Practitioners are advised to evaluate both the nasal and temporal regions of the cornea when assessing 3- and 9-o'clock staining, and they should be hesitant to rely on the amount of bulbar hyperemia for the prediction of the severity of 3- and 9-o'clock staining. Assessment of conjunctival staining could potentially be a useful tool in clinical practice to identify patients at risk of developing 3and 9-o'clock staining. The lack of correlation between corneal staining and lens comfort stresses the need for regular contact lens check-ups in GP lens wearers. It appears that the simple registration of the presence or absence of ocular symptoms is a better predictor of 3- and 9-o'clock staining than using VAS comfort scores.

Key words: GP contact lenses, 3- and 9-o'clock corneal staining, bulbar hyperemia, conjunctival staining, lens comfort 
The term 3- and 9-o'clock staining, ${ }^{1}$ (also called peripheral corneal staining, ${ }^{2}$ peripheral corneal desiccation, ${ }^{3} 4$ and 8 o'clock staining, ${ }^{4}$ juxtaposition staining ${ }^{5}$ or persistent nasal and temporal stippling ${ }^{6}$ ) describes the condition of corneal epithelial staining in the nasal and temporal regions of eyes wearing rigid or gaspermeable (GP) contact lenses. This condition has been reported in the literature since the introduction of rigid corneal lenses in the early $1950 \mathrm{~s}^{5-7}$ Some degree of 3- and 9-o'clock staining has been described in as many as $80 \%$ of GP lens wearers, ${ }^{8}$ but it is estimated to be of clinical significance in about $10-15 \%$. As with any other condition, 3- and 9-o'clock staining greater than grade two should be considered as clinically significant. ${ }^{9,10}$ In severe cases, 3- and 9-o'clock staining may cause corneal scarring if the anterior limiting membrane is affected. ${ }^{11}$ Also, localized thinning of the cornea in the limbal area (dellen) and a vascularized limbal keratitis may develop. ${ }^{12}$

The clinical consequence of low levels of 3- and 9-o'clock corneal staining is hard to define. A clear association between dry eye, punctate corneal staining and tear film integrity has been reported, ${ }^{13-16}$ but no studies directly analyzing the possible risks involved in 3- and 9-o'clock staining are available. Recently, corneal staining and its clinical relevance have received a lot of attention. Superficial epithelial damage caused by contact lens storage solutions has been linked to infectious and inflammatory events by some, ${ }^{17-19}$ whereas others have disputed this relationship, stating that the mechanism behind corneal infectious disease is complex and multifactorial and cannot simply be attributed to corneal staining. ${ }^{20,21}$ It has been suggested that superficial punctate patterns of fluorescein staining (as seen in solution toxicity) should be viewed differently from non-superficial coalesced injury-related staining. ${ }^{20}$

One of the key tasks for contact lens clinicians in daily practice is to evaluate the presence and amount of corneal staining, in order to assess the physiological status of the corneal epithelium. ${ }^{20}$ As a general rule in clinical practice it is accepted that 'brighter is worse' and 'more is bad' when it comes to corneal staining. ${ }^{21}$ In many studies, the presence of corneal staining is considered a complication of lens wear and generally it is believed that it is better to avoid 3-and 9-o'clock staining at any time if possible, since it represents an alteration in corneal integrity caused by contact lens wear. ${ }^{14}$ The condition of 3- and 9-o'clock staining is also believed to be a common reason for drop-out in lens wearers. ${ }^{22,23}$

Apart from corneal staining, bulbar hyperemia is frequently mentioned as a secondary sign of 3- and 9-o'clock staining. ${ }^{23,24}$ In addition, anecdotal clinical observation suggests that patients exhibiting 3- and 9-o'clock staining may present with 
significant levels of conjunctival fluorescein staining, but this relationship has not been investigated previously.

The condition of 3- and 9-o'clock staining has been cited to give rise to a wide array of symptoms, usually summarized as 'a decrease in lens comfort'; however, the magnitude of these symptoms is believed to not always correlate well with the amount of corneal staining. ${ }^{10,12-15}$ A reduction in lens comfort during the course of the day has been reported clinically. ${ }^{25}$

This study analyses the relationship between the signs and symptoms related to 3and 9-o'clock staining, such as corneal staining, bulbar hyperemia, conjunctival staining and lens comfort. In addition, other variables such as subject age, gender, wearing time per day, days of lens wear per week and hours of lens wear at the time of examination are evaluated to investigate any relationships with the signs and symptoms that accompany 3-and 9-o'clock staining.

\section{Methods}

\section{Subjects and Lens Wear}

This study involved 81 subjects. Of these, 55 were GP contact lens wearers, both with and without 3- and 9-o'clock staining, from the database of a large contact lens practice in the Netherlands. The control group consisted of 26 randomly selected non-lens wearing patients of the same practice. The GP lens wearers included in this study were long term, successful contact lens wearers who were seen as part of a regular checkup schedule.

To investigate the inter-relationships between the signs related to 3-and 9-o'clock staining (corneal staining, bulbar hyperemia and conjunctival staining), 162 eyes were used for analysis while for all other variables the number of subjects $(n=81)$ was used for analysis.

Information about the ocular history was obtained by interviewing the subjects. All tested eyes were free of pathological eye conditions, including pathological dry eye conditions. Subjects were asked to wear their lenses for a minimum of one hour on the day of the examination visit. The measurements took place between 9am and $6 \mathrm{pm}$. All procedures in this study were conducted in accordance with the Declaration of Helsinki (2000), and all subjects signed an informed consent form prior to participation in the study. 


\section{Signs}

A detailed slitlamp examination was performed using fluorescein (Fluorets, Chauvin), cobalt blue light and a Wratten \#12 yellow barrier filter. A specially designed image grading scale for 3- and 9-o'clock staining, developed at the University of Maastricht, was used to grade all eyes in decimals on a $0-4$ point scale. This grading scale was developed based on currently available descriptive scales for 3- and 9$o^{\prime}$ clock staining and general grading scales for corneal staining. ${ }^{26}$

Both the nasal and temporal regions of the cornea were rated separately. The highest score of the two was used to determine the amount of staining for that eye. The maximum amount of staining in both eyes of each subject was rated by two investigators separately. If different results were found between the two investigators, the average of the two ratings was used for further analysis.

Three groups were identified: non-lens wearers formed the control group (group I), GP lens wearers with less than grade 1 staining in the three or nine o'clock position of the cornea were assigned to group II and GP lens wearers with significant 3-and 9-o'clock corneal staining (grade 1 or more in at least one eye) were assigned to group III.

Bulbar hyperemia was rated in decimals using the continuous Efron 0-4 point grading scale. ${ }^{27}$ Both the nasal and temporal regions of the eye were rated separately and the highest score of the two was used to determine the amount of bulbar hyperemia for that eye.

To mark the presence of conjunctival staining, all eyes were examined using fluorescein utilizing the same method as described for corneal staining. Pooling of fluorescein in conjunctival folds was not considered to be true conjunctival staining. Eyes with grade 1 or higher on the Efron grading scale ${ }^{27}$ were considered as having conjunctival staining, otherwise they were labeled as having no conjunctival staining.

\section{Symptoms}

Ocular comfort and lens comfort were evaluated in two ways. First, visual analogue scale (VAS) scores on a $0-10$ scale $(0=$ pain/minimal eye comfort, $10=$ maximum eye comfort/not feeling anything) were obtained for each eye separately. VAS scores from the two eyes were averaged for further analysis. Secondly, subjects were simply asked if they had any ocular symptoms, including dryness, burning and/or itching. If any of these symptoms were reported, then the subject was marked as symptomatic, otherwise this subject was marked as asymptomatic. To evaluate the 
relationship between comfort ratings and the signs of 3- and 9-o'clock staining, the maximum score of corneal staining, bulbar hyperemia and conjunctival staining of the right and left eyes was used for analysis.

\section{Data Analysis}

Statistical analysis of the data was performed using Statistical Package for Social Sciences (SPSS) version 11.5. Because of the mostly non-Gaussian nature of the distribution of data, the non-parametric Mann-Whitney test and (if indicated in the text) chi-square test were used for statistical analysis. The ANOVA test was used to analyze the mean age of subjects across the three groups. Power analysis was used to ensure an adequate sample size. ${ }^{28} \mathrm{~A}$ critical $\mathrm{p}$ value of 0.05 was used to denote statistical significance. Correlations between variables were analyzed by calculating Spearman's rho. All data are presented as median and range, unless otherwise stated.

\section{Results}

\section{Subjects and Lens Wear}

The mean age $( \pm S D)$ of subjects was $40.7 \pm 13.4$ years (range 14.9 to 72.4 years). Group I consisted of 26 subjects ( $33.8 \pm 10.9$ years, range 18.5 to 56.6), group II consisted of 25 subjects $(41.9 \pm 16.1$, range 14.9 to 72.4$)$, and 30 subjects ( $42.9 \pm$ 11.2 , range 21.6 to 68.0 ) formed group III. A statistically significant difference for age was found between these groups $(\mathrm{p}=0.02)$. The proportion of male to female subjects in the study was $31 \%$ male to $69 \%$ female, which is a realistic reflection of the gender ratio of contact lens wearers around the world. ${ }^{29}$ No statistically significant difference for gender was found across groups.

In the contact lens wearing group, the median number of years that subjects had been wearing GP contact lenses was 22 (1.5 to 38.0) years. The reported median lens wearing time per day was 16 (6.0 to 18.0 ) hours and the number of wearing days per week was 7.0 (5.5 to 7.0) days. The median wearing time of the lenses on the day of examination was 3.5 (1.0 to 11.0) hours. An overview of these variables, subdivided into groups I, II and III, is presented in Table 1.

\section{Signs}

\section{Corneal Staining}

The median grade of corneal staining (Table 2) in group I was 0.2 , while in group II this was grade 0.3 , and grade 1.5 in group III. Median grades of corneal staining nasally and temporally were found to be similar (grade 0.4 ). The correlation be- 
tween nasal and temporal staining in group I was $\mathrm{r}=0.69(\mathrm{p}<0.01)$, in group II the correlation was $\mathrm{r}=0.55(\mathrm{p}<0.01)$ and $\mathrm{r}=0.12(\mathrm{p}=0.36)$ in group III.

No gender difference in the amount of 3- and 9-o'clock staining was found, and no correlation was found between corneal staining and subject age, years of lens wear, lens wearing time per day, or lens wearing days per week. There was a low but significant correlation between staining grade and hours of lens wear at the time of examination $(\mathrm{r}=0.32 ; \mathrm{p}=0.02)$.

TABLE 1.Summary of subject-related variables in relation to 3- and 9-o'clock staining for group I (non-lens wearers), group II (GP lens wearers with grade 1 or less corneal staining) and group III (GP lens wearers with more than grade 1 corneal staining)

\begin{tabular}{lcccc}
\hline & $\begin{array}{c}\text { Group I } \\
\mathrm{n}=26\end{array}$ & $\begin{array}{c}\text { Group II } \\
\mathrm{n}=25\end{array}$ & $\begin{array}{c}\text { Group III } \\
\mathrm{n}=30\end{array}$ & $\mathrm{p}$ value \\
\hline $\begin{array}{l}\text { Age of subject } \\
\text { (mean } \pm \text { SD) }\end{array}$ & $33.8 \pm 10.9$ & $41.9 \pm 16.1$ & $42.9 \pm 11.2$ & $\mathrm{p}=0.02$ \\
$\begin{array}{l}\text { Gender ratio } \\
\text { (male:female) }\end{array}$ & $38: 62$ & $20: 80$ & $33: 67$ & $\mathrm{p}=0.54$ \\
$\begin{array}{l}\text { Years of contact lens wear } \\
\text { (median, range) }\end{array}$ & $\mathrm{n} / \mathrm{a}$ & $26(1.5$ to 38.0$)$ & $20.5(2.0$ to 37.0$)$ & $\mathrm{p}=0.17$ \\
$\begin{array}{l}\text { Hours of wearing time per day } \\
\text { (median, range) }\end{array}$ & $\mathrm{n} / \mathrm{a}$ & $16(12.0$ to 18.0$)$ & $16(6.0$ to 18.0$)$ & $\mathrm{p}=0.48$ \\
$\begin{array}{l}\text { Days of lens wear per week } \\
\text { (median, range) }\end{array}$ & $\mathrm{n} / \mathrm{a}$ & $7.0(5.5$ to 7.0$)$ & $7.0(6.0$ to 7.0$)$ & $\mathrm{p}=0.58$ \\
$\begin{array}{l}\text { Hours of lens wear at time of exami- } \\
\text { nation (median, range) }\end{array}$ & $\mathrm{n} / \mathrm{a}$ & $2.5(1.0$ to 10.0$)$ & $6.0(1.0$ to 11.0$)$ & $\mathrm{p}=0.01$ \\
\hline
\end{tabular}

\section{Bulbar Hyperemia}

The median grade of bulbar hyperemia in groups I and II was 0.8 , and 1.0 in group III (Table 2). The correlation between bulbar hyperemia in the nasal and temporal regions of the eye in group I was $\mathrm{r}=0.80(\mathrm{p}<0.01)$, in group II $\mathrm{r}=0.51$ $(\mathrm{p}<0.01)$, and $\mathrm{r}=0.73(\mathrm{p}<0.01)$ in group III.

No correlation was found between the amount of bulbar hyperemia and the amount of corneal staining in each group separately. However a low but significant correlation between these two variables was found when data for all three groups were combined $(\mathrm{r}=0.23, \mathrm{p}<0.01)$. 
TABLE 2. Summary of signs and symptoms accompanying 3- and 9-o'clock staining in group I (non-lens wearers), group II (GP lens wearers with grade 1 or less corneal staining) and group III (GP lens wearers with more than grade 1 corneal staining)

\begin{tabular}{lccc}
\hline Signs & $\begin{array}{c}\text { Group I } \\
\mathrm{n}=52 \text { eyes }\end{array}$ & $\begin{array}{c}\text { Group II } \\
\mathrm{n}=50 \text { eyes }\end{array}$ & $\begin{array}{c}\text { Group III } \\
\mathrm{n}=60 \text { eyes }\end{array}$ \\
\hline $\begin{array}{l}\text { Grade corneal staining } \\
\text { (median, range) }\end{array}$ & $0.2(0$ to 0.8$)$ & $0.3(0$ to 0.9$)$ & $1.5(1.0$ to 4.0$)$ \\
$\begin{array}{l}\text { Grade bulbar hyperemia } \\
\text { (median, range) }\end{array}$ & $0.8(0.5$ to 1.5$)$ & $0.8(0.2$ to 1.7$)$ & $1.0(0.3$ to 3.0$)$ \\
$\begin{array}{l}\text { Conjunctival staining } \\
\text { (\% of subjects) }\end{array}$ & $0 \%$ & $14 \%$ & $40 \%$ \\
\hline $\begin{array}{l}\text { Symptoms } \\
\text { VAS comfort scores }(0-10)\end{array}$ & Group I & Group II & Group III \\
(median, range) & $\mathrm{n}=26$ & $\mathrm{n}=25$ & $9.1(4.2$ to 10$)$ \\
$\begin{array}{l}\text { Symptomatic subjects } \\
\text { (\% of subjects) }\end{array}$ & $8.9(4.9$ to 10$)$ & $9.7(0$ to 10$)$ & $53 \%$ \\
\hline
\end{tabular}

No gender difference in bulbar hyperemia was found, and no correlation was seen between bulbar hyperemia and subject age, years of lens wear, lens wearing time per day, number of wearing days per week or hours of lens wear at the time of examination.

\section{Conjunctival Staining}

Conjunctival staining was observed in $18 \%$ of eyes. No cases of conjunctival staining were found in group I, while $14 \%$ of subjects in group II and $40 \%$ of subjects in group III showed conjunctival staining (Table 2). The median grade of corneal staining was 2.0 ( 0.2 to 4.0$)$ in eyes with conjunctival staining, while this was only 0.2 ( 0 to 3.0$)$ in eyes without conjunctival staining $(\mathrm{p}<0.01)$.

The median grade of bulbar hyperemia in eyes with conjunctival staining was 0.9 (0.3 to 3.0) and 0.9 ( 0.2 to 2.5$)$ in eyes without conjunctival staining ( $\mathrm{p}=0.91)$.

In relation to gender, $14 \%$ of male subjects showed conjunctival staining whereas $26 \%$ of the female subjects showed conjunctival staining $\left(\chi^{2}, \mathrm{p}=0.07\right)$. The age of subjects with conjunctival staining was higher than subjects without conjunctival staining (mean $46.4 \pm \mathrm{SD} 12.4$ years versus $38.4 \pm 13.2$, $\mathrm{p}<0.01$ ). No difference was found between subjects with and without conjunctival staining with regard to years of lens wear, lens wearing time per day, lens wearing days per week or hours of lens wear at the time of examination. 


\section{Symptoms}

\section{VAS Comfort Scores}

The median reported ocular comfort on a 0-10 point scale in non-lens wearers (group I) was 8.9, while in group II this was 9.7 and 9.1 in group III (Table 2). No correlation between VAS comfort scores and corneal staining was found in any group, nor when data across groups were combined.

No correlations were found between comfort scores and the grade of bulbar hyperemia in groups I or III. A low but significant correlation was revealed in group II $(r=0.45, p=0.02)$. No statistically significant difference in comfort scores were seen between subjects with and without conjunctival staining: 8.9 (0.2 to 10) versus 9.8 ( 4.4 to 10 ) respectively $(\mathrm{p}=0.14)$. No difference in comfort scores was found between male and female subjects. No correlations were found between comfort scores and subject age, lens wearing days per week and hours of lens wear at the time of examination, while low but significant correlations were found between comfort scores and years of lens wear $(r=0.30, p=0.02)$ and lens wearing time per day $(\mathrm{r}=0.30, \mathrm{p}=0.04)$.

\section{Presence of Ocular Symptoms}

Evaluation of ocular comfort and lens comfort by simply recording the presence or absence of ocular symptoms revealed some differences between groups. In groups I and II, $42 \%$ of subjects were symptomatic while this was $53 \%$ in group III (Table 2).

A median grade of 0.8 (0 to 4.0) corneal staining was found in symptomatic subjects versus a grade 0.4 ( 0 to 2.8 ) in asymptomatic subjects $(\mathrm{p}=0.07)$. In group I the difference in staining between symptomatic and asymptomatic subjects was minimal: 0 (0 to 1.3 ) versus 0 ( 0 to 0.8$)$ respectively. This was also the case in group II: 0.4 (0 to 1.2 ) versus 0.3 (0.1 to 1.1 ) respectively. However, in group III a significant difference was found between symptomatic and asymptomatic subjects: grade 2.7 (1.3 to 4.0$)$ versus 1.5 ( 1.8 to 2.8$)$ respectively $(\mathrm{p}<0.01)$.

Bulbar hyperemia in the symptomatic group was not statistically significantly different from that in the asymptomatic group, either overall or in each group separately. Symptomatic subjects presented more often with conjunctival staining than asymptomatic subjects: $26 \%$ of the subjects with symptoms showed conjunctival staining versus $9 \%$ of the subjects without symptoms $\left(\chi^{2}, p=0.04\right)$. 
No statistically significant differences between symptomatic and asymptomatic subjects were found for gender, subject age, years of lens wear, wearing days per week, wearing time per day or hours of lens wear at the time of examination.

\section{Discussion}

The condition 3- and 9-o'clock staining is characterized by staining in the nasal and temporal regions of the cornea. Although the median grades of corneal staining nasally and temporally were found to be of a similar level and highly correlated in Groups I and II, the correlation between nasal and temporal staining was found to be very low and not significant in Group III subjects with substantial amounts of 3- and 9-o'clock staining. Therefore, it is suggested that both the nasal and temporal regions of the cornea should be evaluated when grading this condition.

Overall, our results show a statistically significant but low correlation $(\mathrm{r}=0.32$, $\mathrm{p}=0.02$ ) between the level of corneal staining and hours of lens wear prior to examination. There was also a statistically significant difference in hours of lens wear prior to examination ( $\mathrm{p}=0.01$ ) between groups II and III (Table 1). This leaves open the possibility that subjects in Group II may have progressed to Group III staining levels if they had been seen later in the day or after a longer period of lens wear. Corneal staining may be related to the time of day in dry eye patients. ${ }^{30}$ From clinical observations, a decrease in comfort during the day while wearing lenses has been reported, ${ }^{25}$ although there are no controlled studies to support this. For logistical reasons we did not standardize the time of day or hours of lens wear prior to examination, and this limitation of our study should be controlled in future research. In this study subjects were seen in a private practice, often as part of a regular check up. Some patients were not able or willing to adjust for longer wearing times. Routine corneal staining was analyzed after a minimum of one hour of lens wear; the median prior wearing time of the lenses on the day of examination was 3.5 hours with a range of 1.0 to 11.0 hours. The question whether the time of examination during the day is critical for practitioners assessing GP lens wearers for 3- and 9-o'clock staining is therefore not clear in this study. Nevertheless, the relationships between signs and symptoms of 3- and 9-o'clock staining are likely to be minimally affected by this limitation.

Bulbar hyperemia is mentioned in the literature as the main secondary sign of 3and 9-o'clock staining. ${ }^{23}{ }^{24}$ The amount of bulbar hyperemia in GP lens wearers with substantial 3- and 9-o'clock staining (group III) was only slightly higher than in group I (non-lens wearers) and group II (GP lens wearers with less than grade 1 corneal staining). The correlation between corneal staining and bulbar hyperemia 
was statistically significant, but very low. Based on these results it is suggested that practitioners should be hesitant to use the amount of bulbar hyperemia to predict the severity of 3- and 9-o'clock staining. Duench et al. ${ }^{31}$ found a diurnal variation in hyperemia in normal subjects, the hyperaemia being greatest shortly after waking. This was not taken into account in this study, but should be controlled for in future studies.

In eyes with conjunctival staining, statistically significantly more corneal staining was found than in eyes without conjunctival staining, confirming anecdotal clinical observations. Symptomatic subjects also presented more often with conjunctival staining than asymptomatic subjects. One should keep in mind that the age of subjects with conjunctival staining was higher than those without conjunctival staining, and age may thus be a contributing factor to this finding.

In recent years it has become increasingly apparent that bulbar conjunctival anomalies, including conjunctival staining, are associated with ocular dryness. ${ }^{32-35}$ To the best of our knowledge the occurrence of conjunctival staining in patients with 3- and 9-o'clock staining has never been investigated. The results of this study indicate that observing the presence of conjunctival staining could be a useful adjunct tool in clinical practice when assessing corneal staining, although further research is needed in this regard. In addition, it might be of interest to investigate the potential of other conjunctival dyes for the evaluation of 3-and 9-o'clock staining, such as rose bengal and lissamine green. Both dyes have proven their usefulness in clinical practice for the detection of dry eye related conditions. ${ }^{36-39}$

It has been reported that the signs of 3- and 9-o'clock staining are poorly related to the level of lens comfort. ${ }^{10,12-15}$ No correlation between VAS comfort scores and the amount of corneal staining was found in this study. Also, no relationship was found between VAS comfort scores and bulbar hyperemia or between VAS comfort scores and conjunctival staining. The simple registration of the presence or absence of ocular symptoms proved to be a better predictor than VAS comfort scores for the presence of ocular signs, particularly in group III where symptomatic subjects showed significantly more corneal staining than asymptomatic subjects. This emphasizes that routine contact lens examinations are required for all GP lens wearers since corneal staining seems not necessarily to be accompanied by discomfort.

Looking at the absolute values of comfort in non-lens wearers and lens wearers, it is of interest to note that the level of comfort was within the same range in all three groups. This similarity in comfort between GP wearers and non-wearers may ap- 
pear incongruous, but it should be kept in mind that all of our subjects were existing, successful, well-adapted GP lens wearers, who were apparently comfortable with their mode of lens wear. Subjects did not compare lens wear to non-lens wear. Within the GP lens-wearing group, subjects with significant amounts of staining did show a slightly lower comfort level than subjects without significant staining, although that this was not found to be statistically significant.

\section{Conclusions}

With regard to the signs accompanying 3-and 9-o'clock staining, it was found that the correlation between corneal staining and bulbar hyperemia was low but significant. More corneal staining was found in eyes with conjunctival staining than those without conjunctival staining, confirming anecdotal clinical observations. No correlation between nasal and temporal staining was found in patients with substantial staining. When looking at symptoms in 3- and 9-o'clock staining, the simple registration of the presence or absence of ocular symptoms was found to be a better predictor of 3- and 9-o'clock staining than using VAS comfort scores.

Routine contact lens examinations are recommended for all GP lens wearers because lens comfort and bulbar hyperemia are poorly correlated with the amount of 3- and 9-o'clock staining. It seems advisable for practitioners to evaluate both nasal and temporal regions of the cornea, and they should be hesitant to use the amount of bulbar hyperemia for prediction of the severity of 3-and 9-o'clock staining. The positive relationship between conjunctival staining and 3- and 9-o'clock staining could prove to be a potentially useful tool in clinical practice to identify patients at risk of developing 3-and 9-o'clock staining. The use of additional variables such as gender, subject age, wearing time per day, days of lens wear per week and hours of lens wear at the time of examination were shown to be of limited value in relation to the assessment of 3-and 9-o'clock staining.

\section{Acknowledgements}

The authors wish to thank Jantine Bos and Rob Faas of Groenhof Optiek in Amstelveen, the Netherlands, and Peter van Etten for their help during the study.

Thanks are also due to Michael Baertschi, Ineke Krijger and Matthew Lampa for their valuable input with regards to this study and paper. 


\section{References}

1. Van der Worp E, De Brabander J, Swarbrick H, Nuijts R, Hendrikse F. Corneal desiccation in rigid contact lens wear: 3- and 9-o'clock staining. Optom Vis Sci 2003;80:280-90.

2. Andrasko G. Peripheral corneal staining: incidence and time course. Contact Lens Spectrum 1990;5(7):59.

3. Schnider C, Terry R, Holden B. Effect of patient and lens performance characteristics on peripheral corneal desiccation. J Am Optom Assoc 1996;67:144-50.

4. Arner RS. Corneal contact lens design by minimal corneal insult. J Am Optom Assoc 1969;40:308-9.

5. Barabas R, Fontana A. Juxtaposition staining. Contacto 1967;11:3-6.

6. Graham R. Persistent nasal and temporal stippling. Contacto 1968;12:20-12.

7. Steele E. Observations on the fitting of corneal contact lenses. Am J Optom Arch Am Acad Optom 1959;36:194-9.

8. Solomon J. Causes and treatments of peripheral corneal desiccation. Contact Lens Forum 1986;11:30-6.

9. Efron N. Grading scales for contact lens complications. Ophthalmic Physiol Opt 1998;18:1826.

10. Terry RL, Schnider CM, Holden BA et al. CCLRU standards for success of daily and extended wear contact lenses. Optom Vis Sci 1993;70:234-43.

11. Doshi S. Corneal anatomy, physiology and response to wounding. In: Naroo SA, ed. Refractive Surgery: A Guide to Assessment and Management. London: Butterworth-Heinemann, 2004:1726.

12. Grohe R, Lebow K. Vascularized limbal keratitis. Int Contact Lens Clin 1989;16:197-209.

13. Yokoi N, Takehisa Y, Kinoshita S. Correlation of tear lipid layer interference patterns with the diagnosis and severity of dry eye. Am J Ophthalmol 1996;122:818-24.

14. Nichols KK, Mitchell GL, Simon KM, Chivers DA, Edrington TB. Corneal staining in hydrogel lens wearers. Optom Vis Sci 2002;79:20-30.

15. Nichols KK, Nichols JJ, Lynn Mitchell G. The relation between tear film tests in patients with dry eye disease. Ophthalmic Physiol Opt 2003;23:553-60.

16. Bourcier T, Acosta MC, Borderie V et al. Decreased corneal sensitivity in patients with dry eye. Invest Ophthalmol Vis Sci 2005;46:2341-5.

17. Norn MS. Micropunctate fluorescein vital staining of the cornea. Acta Ophthalmol (Copenh) 1970;48:108-18.

18. Li SL, Ladage PM, Yamamoto T, Petroll WM, Jester JV, Cavanagh HD. Effects of contact lens care solutions on surface exfoliation and bacterial binding to corneal epithelial cells. Eye Contact Lens 2003;29:27-30.

19. Cavanagh HD. Fusarium, contact lens solutions, and patient compliance: a tangled, critical web. Eye Contact Lens 2006;32:255.

20. Ward KW. Superficial punctate fluorescein staining of the ocular surface. Optom Vis Sci 2008;85:8-16.

21. Levy B. Superficial corneal "staining"-clinical observation and risk assessment. Eye Contact Lens 2007;33:165-6.

22. Tomlinson A. Contact lens-induced dry eye. In: Tomlinson A, ed. Complications in Contact Lens Wear. St. Louis: Mosby, 1992:195-218.

23. Lowther GE. Dryness, Tears, and Contact Lens Wear: Clinical Practice in Contact Lenses. Boston: Butterworth-Heinemann, 1997:84-90.

24. Snyder C. Dry eye symptoms and contact lens wear. In: Silbert J, ed. Anterior Segment Complications of Contact Lens Wear. New York: Churchill Livingstone, 1994:213-20. 
25. Lebow K. Reduce three-and-nine corneal staining with moderate edge lift profiles. Contact Lens Spectrum 1990;5(7):20-33.

26. Van der Worp E, De Brabander J, Swarbrick H, Hendrikse F. Eyeblink frequency and type in relation to 3- and 9-o'clock staining and gas permeable contact lens variables Optom Vis Sci 2008;85:857-66

27. Efron N, Morgan PB, Jagpal R. Validation of computer morphs for grading contact lens complications. Ophthalmic Physiol Opt 2002;22:341-9.

28. Hulley SB, Cummings SR, Browner WS, Grady D, Hearst N, Newman TB. Designing Clinical Research. Philadelphia: Lippincott Williams \& Wilkins, 2001:65-91.

29. Morgan P, Efron N, Woods $\mathrm{C}$ et al. International contact lens prescribing 2007. Contact Lens Spectrum 2008;23(1):36-41.

30. Begley CG, Chalmers RL, Abetz L et al. The relationship between habitual patient-reported symptoms and clinical signs among patients with dry eye of varying severity. Invest Ophthalmol Vis Sci 2003;44:4753-61.

31. Duench S, Simpson T, Jones LW, Flanagan JG, Fonn D. Assessment of variation in bulbar conjunctival redness, temperature, and blood flow. Optom Vis Sci 2007;84:511-6.

32. Simon P, Jaison SG, Chopra SK, Jacob S. Conjunctival impression cytology in contact lens wearers. Indian J Ophthalmol 2002;50:301-6.

33. Lakkis C, Brennan NA. Bulbar conjunctival fluorescein staining in hydrogel contact lens wearers. CLAO J 1996;22:189-94.

34. Bron AJ, Evans VE, Smith JA. Grading of corneal and conjunctival staining in the context of other dry eye tests. Cornea 2003;22:640-50.

35. Guillon M, Maissa C. Bulbar conjunctival staining in contact lens wearers and non lens wearers and its association with symptomatology. Cont Lens Anterior Eye 2005;28:67-73.

36. Norn MS. Vital staining of cornea and conjunctiva. Fluorescein-rose bengal mixture and tetrazolium-alcian blue mixture. Acta Ophthalmol Suppl 1972;113:3-66.

37. van Bijsterveld OP. Diagnostic tests in the Sicca syndrome. Arch Ophthalmol 1969;82:10-4.

38. Manning FJ, Wehrly SR, Foulks GN. Patient tolerance and ocular surface staining characteristics of lissamine green versus rose bengal. Ophthalmology 1995;102:1953-7.

39. Khurana AK, Chaudhary R, Ahluwalia BK, Gupta S. Tear film profile in dry eye. Acta Ophthalmol (Copenh) 1991;69:79-86. 


\section{2 \\ Evaluation of Contact Lens Related Variables in 3- and 9-O'clock Staining}

Van der Worp E, De Brabander J, Swarbrick H, Hendrikse F. Submitted

Eye Research Institute Maastricht, Department of Ophthalmology Academic Hospital, Maastricht University, The Netherlands (EW, JB, FH) and the School of Optometry and Vision Science, University of New South Wales, Sydney, Australia (HS) 


\begin{abstract}
Purpose. This study evaluates the contact lens related variables that could potentially contribute to the development of 3- and 9-o'clock staining in rigid gas permeable (GP) contact lens wear, to better understand the condition and its etiology. Methods. The study involved 110 eyes of 55 wearers of GP contact lenses, with and without 3- and 9-o'clock staining. Five contact parameter-related variables were investigated: central lens fitting, edge lift, back surface lens design, on-eye lens size (corneal coverage) and lens edge thickness. Three lens performance variables were studied: lens centration, lens movement and surface wettability.

Results. A low grade of 3- and 9-o'clock staining (< grade 2, low staining group) was recorded in 85 eyes while $\geq$ grade 2 staining (high staining group) was seen in 25 eyes. Eyes with a flat fitting lens (based on edge lift and central lens fit) more often showed a high grade staining (39\%) than eyes with an optimal $(15 \%)$ or steep lens fit $(0 \%)$. Large rated lenses more often showed a high grade of staining $(40 \%)$ than optimal $(24 \%)$ or small $(0 \%)$ rated lenses. Also, interpalpebral lens fits more often showed a high grade of staining (45\%) than lid attachment fits (15\%). None of the other variables showed a significant relationship with 3- and 9-o'clock staining.

Conclusions. Flat lens fits, interpalpebral lens fits and large lenses appear to be risk factors for the development of 3-and 9-o'clock staining, but these cannot be seen as isolated factors. The results of this study point to more than one possible etiology for 3- and 9-o'clock staining, but etiologies based on reduced tear volume and eyeblink inhibition appear to be most strongly supported. Further analysis, focusing specifically on tear volume and eyeblink behavior in GP lens wear, is needed to interpret these findings in more detail.
\end{abstract}

Key words: GP contact lenses, 3- and 9-o'clock corneal staining, lens parameters, lens performance 
The term 3- and 9-o'clock staining describes the condition of corneal epithelial staining in the nasal and temporal regions of eyes wearing GP contact lenses. ${ }^{1}$ This condition has been reported in the literature since the introduction of rigid corneal lenses in the early $1950 \mathrm{~s}^{2-4}$ Some degree of 3- and 9-o'clock staining has been described in as many as $80 \%$ of GP lens wearers, ${ }^{5}$ but it is estimated to be of clinical significance in about $10-15 \% .5,6$

Since the first reports on 3- and 9-o'clock staining, various contact lens related factors have been suggested in the literature that could potentially play a role in the development of the condition and/or serve as management options. These factors can be categorized into eight main groups. Five of these relate to lens parameters, including lens edge lift, back optic zone radius (BOZR), back surface design, lens diameter and the lens edge thickness/shape. Three other factors are related to lens performance, including centration of the lens, lens movement and surface wettability. ${ }^{1}$

The literature is not unanimous about which lens related variables are involved in the development of 3- and 9-o'clock staining, and how these should be optimized to manage 3- and 9-o'clock staining. ${ }^{1}$ For example, there is controversy on whether lenses should be fitted flatter or steeper than corneal contour, ${ }^{6-10}$ and whether they should be made larger or smaller. ${ }^{6,8,10-12}$ The aim of this study is to evaluate the key contact lens related variables that potentially contribute to the development of 3and 9-o'clock staining in order to better understand the condition and its etiology.

\section{Methods}

\section{Subjects and Lens Wear}

This study involved 110 eyes of 55 GP contact lens wearers, both with and without 3- and 9-o'clock staining, from the database of a large contact lens practice in the Netherlands. The lens wearers included in this study were long term, successful contact lens wearers who were seen as part of a regular checkup schedule. All procedures in this study were conducted in accordance with the Declaration of Helsinki (2000), and all subjects signed an informed consent form prior to participation in the study.

Information about the ocular history was obtained by interviewing the subjects. All tested eyes were free of pathological eye conditions, including pathological dry eye conditions. No additional dry eye testing was performed. Subjects were asked to wear their lenses for a minimum of one hour on the day of the examination visit. The measurements took place between $9 \mathrm{am}$ and $6 \mathrm{pm}$. Inclusion criteria only al- 
lowed wearers of rotationally symmetric (non-toric) GP lenses to enter the study. Lens parameters were collected from the subjects' records.

\section{Corneal Staining}

A detailed slitlamp examination was performed using fluorescein, cobalt blue light and a Wratten \#12 yellow barrier filter.

A specially designed image grading scale for 3- and 9-o'clock staining, developed at the University of Maastricht, was used to grade all eyes in decimals on a 0-4 point scale. This grading scale was developed based on currently available descriptive scales for 3- and 9- o'clock staining and general grading scales for corneal staining. ${ }^{13}$

Both the nasal and temporal regions of the cornea were graded in a masked fashion. The highest score of the two was used to determine the amount of staining for that eye. This grading was performed by two investigators separately. If different results were found between the two investigators, the average of the two gradings was used for further analysis.

Typically, grade 2 on $0-4$ clinical grading scales is accepted as a critical clinical cutoff point for the management of contact lens related conditions. ${ }^{14,15} \mathrm{Up}$ to grade 2 is considered to be within normal limits, whereas with grade 2 or higher clinical action usually is indicated. In this study, subjects were categorized as either having $<$ grade 2 staining or $\geq$ grade 2 staining and referred to as 'low' and 'high' staining groups respectively.

\section{Lens Parameters}

To analyze specific contact lens variables that could be related 3- and 9-o'clock staining, an on-eye video recording of each contact lens and its fluorescein pattern was generated with a CCD camera (Mitsubishi CCD-100E) and a DVD recorder (Panasonic DMR E30). The same slitlamp technique (including the use of fluorescein) was used as described for observing corneal staining.

BOZR and edge lift were amongst the key lens parameters identified from the literature as critical for the management of 3- and 9-o'clock staining. ${ }^{1}$ Without any information about the shape of the cornea, however, absolute values of BOZR and edge lift are of limited value when evaluating lens fit and its effect on corneal physiology. Therefore, in this study the lens-to-cornea fit was analyzed on-eye by assessing the tear layer thickness centrally, mid-peripherally and at the edge of the lens. 
The lens was held in a static, central position on the cornea and both the horizontal and vertical meridians of the lens fit were analyzed. Tear layer thickness was graded on a five point scale as either optimal (0), slightly too thick/thin (+/-) but acceptable (grade 1) or excessively too thick/thin (unacceptable, grade 2). An example of a GP contact lens tear layer thickness assessment is shown in Fig. 1.

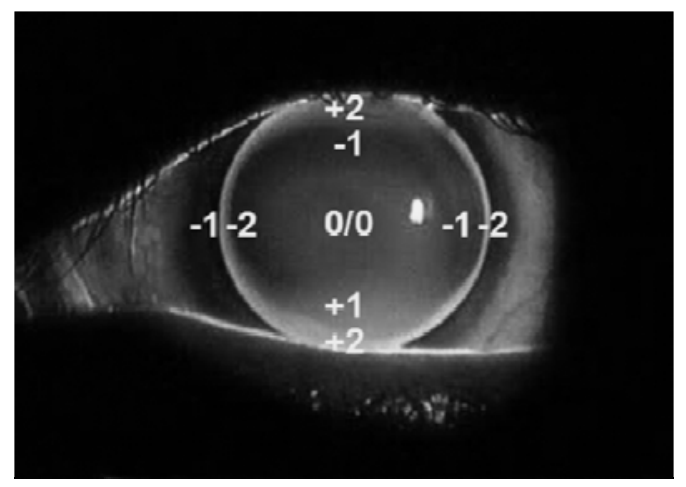

FIGURE 1. Example of tear layer thickness assessment centrally, mid-peripherally and at the edge of the lens in two directions as either optimal (0), slightly too thick/thin (+/-) but acceptable (grade 1) or excessively too thick/thin (unacceptable, grade 2).

The tear layer thicknesses centrally, mid-peripherally and at the edge of the lens were combined and converted into three categories of lens fit. Lens fits were categorized as a flat fit if an unacceptably thin tear layer $(-2)$ was seen centrally and/or an unacceptably thick $(+2)$ tear layer was seen in the midperiphery or at the edge of the lens. An optimal/acceptable lens fit was characterized by a tear layer thickness of grade $0,+1$ or -1 at all lens locations. Steep lens fits showed an unacceptably thick tear layer $(+2)$ centrally and/or an unacceptably thin $(-2)$ tear layer in the midperiphery or at the edge of the lens.

To analyze the back surface geometry of the lens, lens design of the currently worn contact lens was assigned to one of two groups: multicurve designs (bi-, tri- and tetracurve geometries) or aspheric designs. Also, to test the hypothesis that rotationally symmetric lenses on a with-the-rule astigmatic cornea contribute to the development of 3- and 9-o'clock staining, ${ }^{16,17}$ the amount and type of astigmatism of each individual cornea was determined. This was based on pre-contact lens wear keratometry readings. Corneas with up to $0.5 \mathrm{D}$ of toricity were considered spherical and excluded from analysis in this regard. Three groups were formed based on the astigmatism axis: with-the-rule corneal astigmatism $\left(0^{\circ}\right.$ to $30^{\circ}$ and $151^{\circ}$ to $\left.180^{\circ}\right)$, oblique astigmatism $\left(31^{\circ}\right.$ to $60^{\circ}$ and $121^{\circ}$ to $\left.150^{\circ}\right)$ and against the rule corneal astigmatism $\left(61\right.$ to $\left.120^{\circ}\right)$.

Lens size, or more accurately corneal coverage, was subjectively rated on-eye as optimal, large or small. A corneal coverage ratio of approximately 11:9 between the 
vertical visible iris diameter and total lens diameter was considered optimal. Corneal coverage was the preferred variable to consider (as opposed to analysis of absolute lens diameter) because it reflects best whether the size of the lens is appropriate for a particular cornea.

The effect of edge shape is very complex to analyze, especially in a clinical setting. To a lesser extent the same is true for edge thickness. Edge shape and edge thickness were not specifically analyzed due to the large variation of possibilities and the limitations with regard to evaluating these variables. Instead, the spherical equivalent of the refraction was analyzed in relation to 3- and 9-o'clock staining, based on the assumption that a minus-powered lens would typically have a thicker edge profile than a plus-powered lens.

\section{Lens Performance}

Positioning of the lenses on-eye was assessed horizontally and vertically. Lenses were rated as either positioned centrally on the cornea (optimal, grade 0), moderately decentered (acceptable, grade 1) or excessively decentered (grade 2). A decentration nasally was marked as -ve and temporally as +ve and in the vertical direction an inferior decentration was rated -ve while a decentration to a superior position on the cornea was rated $+\mathrm{ve}$.

To further assess the position of the lens in relation to the upper eyelid, all lens fits were rated as either interpalpebral (the contact lens is positioned between the eyelids during interblink periods) or lid attachment (the lens stays under the upper eyelid between eyeblinks).

The movement of the lenses was rated on a five point scale. The speed and amount of movement of the lens with the downward motion of the upper eyelid upon eyeblinking was rated as either optimal (0), suboptimal but acceptable $(+/$-grade 1$)$ or excessive/minimal (+/- grade 2).

Surface wettability was assessed using a Tearscope (Keeler; Windsor, United Kingdom), ${ }^{18}$ and graded on a three point scale as either grade 0 (good wettability), grade 1 (acceptable wettability) or grade 2 (poor wettability). Acceptable wettability was recorded if only localized areas of the lens surface were non-wetting, while in poor wettability large areas of non-wetting or an entire non-wetting lens surface was noted. Also, because of the suggested relationship between oxygen permeability $(\mathrm{Dk})$ and the surface wettability properties of a contact lens material, ${ }^{6,19,20}$ each lens was categorized as either low $\mathrm{Dk}(\leq 30)$ or high $\mathrm{Dk}(>30)$ based on the nominal $\mathrm{Dk}$ 
value as indicated by the manufacturer. In addition, age of the lenses was analyzed to evaluate the potential decrease in surface wettability with older lenses. ${ }^{21}$

\section{Data Analysis}

Statistical analysis of the data was performed using the Statistical Package for Social Sciences (SPSS) version 11.5. Since the distribution of data was mostly nonGaussian in nature, the chi-square test was used for statistical analysis, unless otherwise stated. Correlations between variables were analyzed by calculating Spearman's rho. Power analysis was used to ensure an adequate sample size..$^{22} \mathrm{~A}$ critical $\mathrm{p}$ value of 0.05 was used to denote statistical significance. All data are presented as median and range, unless otherwise stated.

\section{Results}

\section{Subjects and Lens Wear}

The low staining group comprised 85 eyes $(77 \%)$ with a median grade of corneal staining of 0.5 ( 0.0 to 1.9). The high staining group consisted of 25 eyes (23\%) with a median grade of corneal staining of 2.5 (2.0 to 4.0). The mean ( \pm SD) age of subjects in the low staining group was $42.7 \pm 14.2$ years and $43.0 \pm 11.1$ years in the high staining group, and there was no statistically significant difference between these two groups.

The proportion of male to female subjects was $27 \%$ male versus $73 \%$ female, which is a realistic reflection of the gender ratio of contact lens wearers around the world. ${ }^{23}$ No statistically significant difference for gender was found between staining groups. The subjects had been wearing GP lenses for an average of $19.9 \pm 10.6$ years (range 1.5 to 38 years). No difference in years of contact lens wear was found between the low and high staining groups $(20.2 \pm 11.0$ versus $19.1 \pm 9.1$ years respectively).

The median wearing time of the lenses on the day of examination was 3.5 (1.0 to 11.0) hours. The hours of lens wear prior to examination in the high staining group was greater than in the low staining group (mean $5.9 \pm 3.1$ versus $3.9 \pm 3.0$ hours respectively, t-test, $\mathrm{p}=0.01$ ).

\section{Lens Parameters}

\section{Lens Fit/Tear Layer Thicknesses}

Analysis of the tear layer thickness in the central portion of the lens (horizontally and vertically) showed that in the vast majority of cases (96\%) the tear layer thick- 
ness was rated as either optimal or acceptable (grade 1). When looking at the high and low staining groups, no relationship with central tear layer thickness was found in either direction. Table 1 shows the specific tear layer thickness ratings of all lenses at different locations across the lens.

In the midperiphery of the lens, the tear layer thickness was optimal or acceptable in $92 \%$ of cases in the horizontal direction and in $81 \%$ of cases in the vertical direction. Horizontally, a trend towards thicker mid-peripheral tear layers was found in the high staining group compared with the low staining group $(\mathrm{p}=0.06)$, which was not the case in the vertical meridian $(\mathrm{p}=0.47)$.

TABLE 1. Percentage of lens fits in the low and high staining groups showing various tear layer thickness ratings at the centre, mid-periphery and edge of the lens, in the horizontal $(\mathrm{H})$ and vertical (V) meridians. A rating of 0 indicates optimal tear layer thickness, a rating of $+/-1$ indicates an acceptable tear layer thickness, whereas a rating of $+/-2$ indicates an unacceptable tear layer thickness $(+$ $=$ thick, $-=$ thin)

\begin{tabular}{|c|c|c|c|c|}
\hline \multirow{3}{*}{$\begin{array}{l}\text { Tear layer thickness } \\
\text { Staining group } \\
\text { Meridian }\end{array}$} & \multicolumn{4}{|c|}{ Percentage $(\%)$ of lens fits } \\
\hline & \multicolumn{2}{|c|}{ LOW } & \multicolumn{2}{|c|}{ HIGH } \\
\hline & $\mathrm{H}$ & $\mathrm{V}$ & $\mathrm{H}$ & $\mathrm{V}$ \\
\hline \multicolumn{5}{|l|}{ Centre } \\
\hline-2 & 0 & 1 & 4 & 4 \\
\hline-1 & 12 & 14 & 16 & 32 \\
\hline 0 & 62 & 62 & 60 & 44 \\
\hline+1 & 23 & 22 & 20 & 20 \\
\hline+2 & 3 & 1 & 0 & 0 \\
\hline \multicolumn{5}{|l|}{ Mid-periphery } \\
\hline-2 & 8 & 3 & 0 & 0 \\
\hline-1 & 35 & 26 & 32 & 24 \\
\hline 0 & 56 & 31 & 56 & 20 \\
\hline+1 & 0 & 27 & 4 & 32 \\
\hline+2 & 1 & 13 & 8 & 24 \\
\hline \multicolumn{5}{|l|}{ Lens edge } \\
\hline-2 & 7 & 3 & 4 & 0 \\
\hline-1 & 22 & 6 & 16 & 12 \\
\hline 0 & 51 & 30 & 52 & 24 \\
\hline+1 & 19 & 33 & 12 & 8 \\
\hline+2 & 1 & 28 & 16 & 56 \\
\hline
\end{tabular}

At the edge of the lens, the tear layer thickness was rated optimal or acceptable in $89 \%$ of cases in the horizontal direction, and in $63 \%$ of cases in the vertical meridian. In both the horizontal and vertical directions thicker tear layers were seen significantly more frequently at the lens edge in the high staining group than in the low staining group $(\mathrm{p}=0.03)$. 
Based on the combination of tear layer thicknesses centrally, midperipherally and at the edge of the lens, $58 \%$ of lenses showed an optimal or acceptable overall lens fit, in 36\% of cases a flat lens fit was present and in 6\% a steep lens fit was seen. The median grade of corneal staining in flat lens fits was 1.4 (0.0 to 4.0), while this was 0.7 (0.0 to 3.0) in optimal/acceptable lens fits and 0.2 (0.1 to 1.5) in steep lens fits. Eyes with flat lens fits more often were present in the high staining group (39\%) than eyes with optimal/acceptable lens fits (15\%) or eyes with steep lens fits $(0 \%)$, as shown in Figure 2. The relationship between lens fit and staining group was found to be statistically significant $(\mathrm{p}<0.01)$.

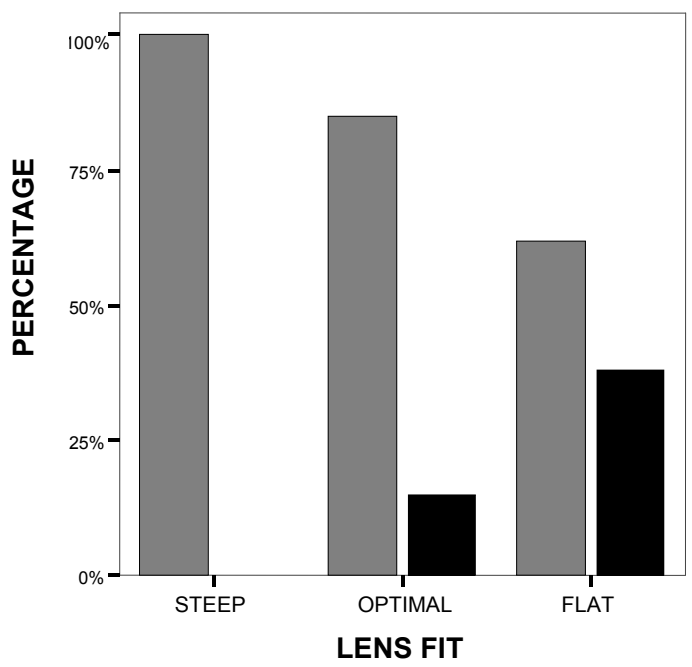

LOW STAINING GROUP

HIGH STAINING GROUP

FIGURE 2. Percentage of subjects with steep, optimal/acceptable and flat lens fittings in the low staining group (grey bars) and the high staining group (black bars).

Lens fit was analyzed separately in the horizontal and vertical directions. Horizontally, 9\% showed a steep lens fit, $86 \%$ had an optimal/acceptable lens fit and 5\% showed a flat lens fit. Of the flat lens fits $(\mathrm{n}=6)$, five eyes $(83 \%)$ were in the high staining group. Of the optimal/acceptable lens fits, $20 \%$ were in the high staining group and of the steep lens fits $10 \%$ were in the high staining group. A statistically significant relationship between staining group and lens fits in the horizontal direction was found $(\mathrm{p}<0.01)$.

In the vertical direction $4 \%$ showed a steep lens fit, $61 \%$ an optimal/acceptable lens fit and $35 \%$ showed a flat lens fit. Of eyes with a flat fit in the vertical direction, $39 \%$ were in the high staining group versus $15 \%$ of eyes with an opti- 
$\mathrm{mal} /$ acceptable lens fit and none of the eyes with a steep lens fit. A statistically significant relationship between staining group and vertical lens fits was seen $(\mathrm{p}=0.01)$.

\section{Back Surface Geometry}

In terms of lens design, $43 \%$ of lenses were identified as having a multicurve design and $57 \%$ as having an aspheric design. In the high staining group, $28 \%$ of the lenses had a multicurve design and $72 \%$ of lenses were of aspheric design, while in the low staining group this was $48 \%$ versus $52 \%$ respectively. The relationship between lens design and staining group was not found to be statistically significant.

To analyze the effect of rotationally symmetric GP lenses on astigmatic corneas, the magnitude of corneal toricity was determined based on central keratometry values. The absolute keratometry measures along either meridian ranged from 7.23 to $8.62 \mathrm{~mm}$ (median $7.94 \mathrm{~mm}$ ) and astigmatism ranged from $0.11 \mathrm{D}$ to $4.35 \mathrm{D}$ (median 1.04D). Overall, 22 eyes were considered 'spherical' (less than $0.5 \mathrm{D}$ of corneal astigmatism), and the median grade of staining in this 'spherical' group was 0.3 (range 0.0 to 3.8). For the 88 'non-spherical' corneas, the distribution of astigmatism type was $84 \%$ with-the-rule astigmatism $(n=74)$, 9\% oblique astigmatism $(n=8)$ and $7 \%$ against-the-rule astigmatism $(n=6)$. The median grade of corneal staining in the with-the-rule astigmatism group was 0.9 (0.0 to 4.0), $0.8(0.0$ to 1.8 ) in the oblique astigmatism group and 0.4 (0.0 to 3.8) in the against-therule astigmatism group. No statistically significant correlations were found between the magnitude or direction of astigmatism and the amount of corneal staining.

\section{Corneal Coverage}

The median lens diameter of all lenses was $9.4 \mathrm{~mm}$ (8.4 to $10.1 \mathrm{~mm}$ ). With regard to corneal coverage, $72 \%$ of lenses were rated as optimal, $13 \%$ as small and $14 \%$ as large. The median grade of corneal staining for small rated lenses was 0.5 (0.0 to 1.4), for optimal rated lenses this was grade 0.8 ( 0.0 to 3.8) and for large rated lenses $1.0(0.0$ to 4.0$)$. In the low staining group $10 \%$ of the lenses were rated as large, $71 \%$ as optimal and $19 \%$ as small. In the high staining group the opposite pattern was seen: $24 \%$ of lenses were rated as large, $76 \%$ as optimal and none of the lenses were rated as small. The relationship between staining group and corneal coverage was statistically significant $(\mathrm{p}=0.03)$.

\section{Edge Thickness}

The spherical equivalent refraction ranged from $-0.50 \mathrm{D}$ to $-10.00 \mathrm{D}$ in myopic subjects (median -3.13D, n=96) and from +2.25D to +7.00D (median +4.00D, $\mathrm{n}=14$ ) in hyperopic subjects. The overall correlation between ametropia and corneal staining was not statistically significant $(\mathrm{r}=-0.02, \mathrm{p}=0.83)$. When divided into myopic 
and hyperopic groups, low correlations were found, which were statistically significant for myopic eyes $(r=-0.24, p=0.02)$, but not for hyperopic eyes $(r=-0.53$, $\mathrm{p}=0.07)$.

\section{Lens Performance}

\section{Centration}

When lens centration in the horizontal direction was evaluated, $67 \%$ of the lenses were found to be positioned centrally on the cornea, $29 \%$ were moderately decentered (grade 1) and 4\% excessively decentered (grade 2). The direction of the decentration was almost equally split between nasal and temporal positioning. In the vertical direction, only $24 \%$ of the lenses were positioned centrally on the cornea: $49 \%$ of lenses showed a grade 1 decentration and $27 \%$ a grade 2 decentration. The direction of the decentration was fairly similar: $40 \%$ of lenses were decentered superiorly and $36 \%$ of lenses were decentered inferiorly.

TABLE 2. Percentages of lenses showing horizontal (H) and vertical (V) lens decentration in the low and high staining groups. A centrally positioned lens was rated as grade 0 (optimal), moderately decentred lenses as grade 1 (acceptable) and unacceptably decentred lenses as grade 2. A decentration temporally was marked as +ve and nasally as -ve, and in the vertical direction a decentration to a superior position was rated +ve while an inferior decentration was rated -ve

\begin{tabular}{|c|c|c|c|c|}
\hline Lens centration & Per & of le & & \\
\hline Staining group & $\mathrm{LO}$ & & $\mathrm{HI}$ & \\
\hline Direction & $\mathrm{H}$ & $\mathrm{V}$ & $\mathrm{H}$ & $\mathrm{V}$ \\
\hline+2 & 1 & 17 & 0 & 8 \\
\hline+1 & 12 & 26 & 4 & 20 \\
\hline 0 & 63 & 19 & 84 & 44 \\
\hline-1 & 21 & 26 & 12 & 16 \\
\hline-2 & 3 & 12 & 0 & 12 \\
\hline
\end{tabular}

In the horizontal direction, $16 \%$ of lenses in the high staining group were decentered while $37 \%$ of lenses in the low staining group were decentered (Table 2). Distribution of nasal versus temporal decentration was found to be similar. No statistically significant relationship between staining groups and centration in the horizontal direction was found.

Vertically, in the high staining group, $56 \%$ showed a decentered lens while $81 \%$ of the lenses in the low staining group showed decentration. Distribution of superior versus inferior decentration was equal. No statistically significant relationship existed between staining group and centration in the vertical direction. 
To further analyze lens centration, the position of the lens in relation to the upper eyelid was assessed during interblink periods. The majority of lens fits (74\%) were lid attachment fits while $26 \%$ showed an interpalpebral fit. Of the lid attachment fits, $15 \%$ showed a high grade of staining, compared with $45 \%$ of the interpalpebral lens fits. This difference was found to be statistically significant $(\mathrm{p}<0.01)$.

\section{Movement}

Optimal lens movement was seen in $43 \%$ of cases, while acceptable lens movement (grade 1) was seen in 50\% of lenses and excessive (grade 2) movement in 7\% of cases. Excessive lens movements all involved slow rather than rapid movement. No statistically significant relationship between staining group and lens movement was found.

\section{Surface Wettability}

Optimal surface wettability was seen in $38 \%$ of cases, and was associated with a median grade of corneal staining of 0.4 (0.0 to 3.8). Acceptable surface wettability was noted in a further $39 \%$ of cases, with a median of grade 1.0 (0.0 to 4.0) corneal staining. In $23 \%$ of cases poor wettability was seen, and this was associated with a median grade 1.0 ( 0.1 to 2.8 ) corneal staining. In the high staining group, $32 \%$ of the lenses showed good wettability while $40 \%$ of the lenses showed acceptable wettability and $28 \%$ poor wettability. The relationship between staining group and surface wettability was not statistically significant.

Low Dk lens materials accounted for 36\% of lenses in this study whereas $64 \%$ of lenses were made of high Dk materials. In the high staining group, $48 \%$ of lenses had a low Dk material while $52 \%$ had a high Dk material. No relationship between lens material Dk and staining group was found.

Age of the lenses that were worn at the time of the examination ranged from 25 days to 8.3 years (median 1.6 years). No correlation was found between lens age and the amount of staining.

\section{Discussion}

\section{Lens Variables}

Of the eight main variables tested, three were found to be related to 3- and 9o'clock staining: more staining was seen with flatter lens fits, larger lenses and interpalpebral lens fits. In addition, myopic subjects demonstrated a low but statistically significant correlation between increasing lens power and corneal staining, presumably because of thicker lens edges. 
Edge lift (or edge clearance) is the most widely cited factor when managing 3- and 9-o'clock staining. ${ }^{7-10}$ However, conflicting evidence is found regarding this in the literature: both increasing (flatter lens fit) ${ }^{9,24}$ and decreasing (steepening the lens fit $)^{7,10}$ the edge clearance has been suggested to remedy 3- and 9-o'clock staining. The same is true for the BOZR: should lenses be fitted flatter or steeper than the flattest keratometry measurement, or in alignment, when managing 3- and 9o'clock staining? ${ }^{3025,26}$ To evaluate these two variables, the post-lens tear layer thickness of all lenses was analyzed in this study. The finding that flat fitting lenses were associated with more 3- and 9-o'clock staining than optimal/acceptable lens fits or steep lens fits seems to point towards avoiding flat fitting lenses in the management of 3- and 9-o'clock staining.

Corneal coverage is an important factor to consider when managing 3- and 9o'clock staining according to the literature, but there is disagreement whether to decrease or increase the total diameter. ${ }^{6,827}$ Illustrating this controversy, nine consultants in a laboratory survey would reduce lens diameter while nine others said they would increase it to manage 3- and 9-o'clock staining. ${ }^{8}$ In our study, corneal coverage did show a positive relationship with 3- and 9-o'clock staining: large rated lenses were associated with more staining than small and optimal rated lenses, suggesting that one should avoid fitting large lenses in 3- and 9-o'clock staining management.

Creating a lid attachment fit has been described by $\operatorname{Korb}^{28}$ and others ${ }^{24,29}$ as being helpful in the management of 3- and 9-o'clock staining. In our study, of all interpalpebral lens fits, $45 \%$ of eyes showed a high grade of staining compared to only $15 \%$ of eyes in the lid attachment group. The findings from this study seem to indicate that the position of the lens on the cornea as such is not the main variable to consider, but rather whether the lens is tucked under the eyelid between eyeblinks or not.

Our results show that lens fit cannot be considered as an individual variable. Avoiding flat fitting lenses as such seems beneficial in the management of 3-and 9o'clock staining, but if a flat lens fit results in a lid attachment fit then this can be useful in the management of 3- and 9-o'clock staining. Based on this, it seems advisable for practitioners to avoid flat fitting lenses in interpalpebral type lens fits. In this study, interpalpebral flat lens fits showed the highest average grade of 3- and 9o'clock staining (grade 2.5, ranging from grade 0.5 to 4.0) of all lens fit-lens type combinations (Table 3). 
A similar argument can be advanced regarding corneal coverage. Smaller lenses seem to be beneficial in the management of 3-and 9-o'clock staining, but larger lenses can create a lid attachment fit, which in turn can be beneficial. Based on this it seems advisable for practitioners to avoid large lenses in interpalpebral fits. In this study, large lenses in interpalpebral fits showed the highest average grade of 3-and 9-o'clock staining (grade 2.5, ranging from grade 0.9 to 4.0 ) of all corneal coverage-lens type combinations (Table 4).

TABLE 3. Median grade of corneal staining (on a scale from $0=$ absent to $4=$ severe) for lid attachment versus interpalpebral lens fits with steep, optimal/acceptable and flat overall lens fittings

\begin{tabular}{llrlll}
\hline & Overall lens fit & $\mathrm{N}$ & $\begin{array}{l}\text { Median } \\
\text { staining }\end{array}$ & $\begin{array}{l}\text { Interquartile } \\
\text { range }\end{array}$ & Overall range \\
\hline Lid attachment & steep & 4 & 0.4 & 1.2 & 0.1 to 1.5 \\
& optimal/acceptable & 51 & 0.6 & 0.9 & 0 to 3.0 \\
& flat & 26 & 1.0 & 2.0 & 0 to 3.0 \\
Interpalpebral & 3 & 0.1 & 1.0 & 0.1 to 1.0 \\
& steep & 13 & 1.3 & 1.7 & 0.2 to 2.5 \\
& optimal/acceptable & 13 & 2.5 & 1.6 & 0.5 to 4.0 \\
\hline
\end{tabular}

TABLE 4. Median grade of corneal staining (on a scale from $0=$ absent to $4=$ severe) for lid attachment versus interpalpebral lens fits with small, optimal and large rated lenses

\begin{tabular}{llrlll}
\hline & Corneal coverage & $\mathrm{N}$ & $\begin{array}{l}\text { Median } \\
\text { staining }\end{array}$ & $\begin{array}{l}\text { Interquartile } \\
\text { range }\end{array}$ & Overall range \\
\hline Lid attachment & small & 14 & 0.4 & 0.7 & 0 to 1.4 \\
& optimal & 57 & 0.6 & 1.3 & 0 to 3.0 \\
& large & 10 & 0.9 & 1.6 & 0 to 3.0 \\
Interpalpebral & 2 & 1.2 & 0.2 & 1.1 to 1.3 \\
& small & 22 & 1.5 & 2.1 & 0.1 to 3.8 \\
& optimal & 5 & 2.5 & 1.8 & 0.9 to 4.0 \\
\hline
\end{tabular}

Apart from the low but statistically significant correlation between myopic power of the lens and corneal staining, none of the other tested variables in this study were found to be related to 3- and 9-o'clock staining. They will be discussed here individually.

Several authors $s^{7,10,30}$ have suggested that in theory an aspheric lens design could have a positive effect on 3- and 9-o'clock staining, since it might follow the shape of the cornea more closely, although De Brabander et al. ${ }^{31}$ were unable to demonstrate this effect in their study. Similarly, no statistically significant relationship between lens design and corneal staining was found in this study. To evaluate the 
effect of lens design on 3- and 9-o'clock staining more accurately, information about the peripheral shape of the cornea would be needed in relation to the lens design. Because current GP lens wearers were evaluated in this study, no reliable baseline topographical data were available and such an evaluation could not be performed.

A non-rotationally symmetric lens on a with-the-rule corneal surface is thought by some to potentially create friction in the three and nine o'clock regions of the peripheral cornea which could lead to 3-and 9-o'clock staining. ${ }^{16}$ In such cases it has been suggested to fit a lens with a toric back surface. ${ }^{16,17}$ In this study, no correlation between the amount of corneal staining and the amount and type of corneal astigmatism was found, although the sample sizes in the oblique and against-therule astigmatism groups were small ( $\mathrm{n}=8$ and 6 , respectively). Central keratometry values were used to establish the amount and type of corneal astigmatism and it has been shown with the use of corneal topographers that corneal astigmatism can increase or decrease towards the periphery, and also that the corneal astigmatism axis may vary towards the periphery. ${ }^{32,33}$ In other words, there may be a relationship between overall corneal astigmatism and 3- and 9-o'clock staining; however central keratometry was inadequate to characterize this fully.

Lens centration has also been cited as a potential key factor in the development and management of 3- and 9-o'clock staining. Typically it is recommended to minimize lens decentration and especially to avoid low riding lenses. ${ }^{10,29,34}$ However, the relationship between corneal staining and lens positioning was not found to be statistically significant in this study, in either the horizontal or vertical direction. This is possibly due to the strong relationship between lens positioning and type of lens fit (lid attachment versus interpalpebral), as discussed before. This indicates that lens centration should not be considered as an independent factor.

Decreasing the edge thickness of a lens and/or improving the edge shape are cited by some as possible management options for 3- and 9-o'clock staining. ${ }^{5,35,36}$ However, the effects of edge shape and edge thickness are very complex to analyze, especially in a clinical setting. It is generally accepted that the periphery of the lens increases in thickness with an increase in myopic correction, and decreases with an increase in hyperopic correction. ${ }^{37}$ However, in high minus lenses the edge of the lens is often lenticulated to reduce edge thickness. Although in this study a statistically significant but low correlation was found between myopic lens power and 3and 9-o'clock staining, this relationship is likely to be complicated by other factors related to peripheral lens design. Based on the findings of this study, therefore, no 
firm statement can be made regarding edge thickness in relation to 3- and 9o'clock staining.

Good lens movement has been mentioned by some to be beneficial in the process of 3- and 9-o'clock staining management, ${ }^{9,38}$ since it is thought that good lens movement promotes the spreading of tear aqueous and mucin which potentially limits peripheral corneal desiccation. The results of our study in relation to lens movement do not provide any support for this hypothesis, as will be further discussed below. As previously mentioned, type of lens fit (lid attachment versus interpalpebral) can be a complicated factor with regards to evaluation of individual lens related variables. This includes lens movement. More lens movement may be expected in lid attachment fits than in interpalpebral lens fits, although no statistically significant difference was found in this regard in our study.

Finally, based on theoretical considerations it has been argued that a clean and well wetting lens material is beneficial in the management of 3- and 9-o'clock staining. ${ }^{10,39,40}$ Although lenses with good wettability showed slightly less 3- and 9o'clock staining than lenses with suboptimal or poor wettability, no statistically significant relationship between the three wettability groups and corneal staining was found. Oxygen permeability and the surface wettability properties of a contact lens are thought to be related, ${ }^{6,19,20}$ but no relationship between oxygen permeability of the material and 3- and 9-o'clock staining was found in this study. Also, age of the lens was evaluated in relation to 3-and 9-o'clock staining since ageing of a lens is believed to have a negative effect on mucous coating and wettability. ${ }^{21}$ Again, no such relationship was found in this study. In summary, it seems that improving the quality of the lens surface and its wettability is of limited value in managing 3-and 9-o'clock staining based on the results of our study.

A statistically significant difference in hours of lens wear prior to examination between the high and the low staining group was found in this study. Theoretically, subjects in the low staining group may have progressed to the high staining group levels if they had been investigated after a longer period of lens wear. The amount of staining that is observed in subjects with dry eye may be related to the time of $\mathrm{day}^{41}$ although no studies have confirmed this finding in relation to 3- and 9o'clock staining. For logistical reasons we did not standardize the time of day or hours of lens wear prior to examination. Subjects were seen in a private practice, often as part of a regular check up, and some patients were not able or willing to adjust for longer wearing times. This limitation should be controlled in future research and this needs to be taken into account as a possible influential factor with regards to the findings of this study. 
It would be of interest to further analyze the lens variables in relation to 3- and 9o'clock staining in more depth, for instance to investigate the effect of flat fitting lenses in interpalpebral lens fits versus the effect of flat fitting lenses in lid attachment fits. However, the sample sizes in individual subcategories were too low in this study for such detailed analysis. Future prospective studies with larger sample sizes representing specific lens fits could possibly differentiate better between the lens related variables in 3- and 9-o'clock staining.

\section{Etiology}

From the literature, a number of theories on the etiology of 3- and 9-o'clock staining can be extracted. ${ }^{1}$ The tear volume theory, also called the tear meniscus theory, described by Holly ${ }^{42}$ and others ${ }^{43,44}$ focuses on the capillary attraction of tears in the corneal area adjacent to the lens edge, where the tear meniscus is created. Due to this capillary attraction, the tear film in the area immediately adjacent to the tear meniscus may become very thin and can break up easily, causing a dry area and subsequent staining.

Another theory is the bridging theory, also called the lid gap theory. Due to a 'bridge' being formed by the upper eyelid between the edge of the lens and the cornea during blinking, a gap is created under the eyelid at the lens edge and mucin coverage of the area under the bridge may be inadequate. Lack of mucin coverage of the nasal and temporal portions of the cornea can result in poor epithelial wetting and corneal staining. ${ }^{5,19,45}$

Decreased eyeblinking during GP lens wear has also frequently been reported as a factor in 3- and 9-o'clock staining. ${ }^{19,36,46}$ The assumption is made that discomfort interferes with eyeblink frequency, ${ }^{23}$ resulting in increased tear evaporation and possibly leading to corneal staining.

According to some authors mechanical factors play a role in the etiology of 3- and 9-o'clock staining. ${ }^{16,17,26}$ This theory postulates that excessive friction of the lens edge against the limbal area can cause 3- and 9-o'clock staining, and with-the-rule corneal toricity is recognized as a risk factor. ${ }^{12,49}$

The finding in this study that more 3- and 9-o'clock staining was associated with flat fitted lenses seems to support more than one theory on the etiology of the condition. Flat fitted lenses may attract more tear volume to the area underneath the periphery of the lens, which is in line with the tear volume theory. The association between increased staining and a flat lens fit also seems to support the bridge theory: the gap between the upper eyelid and the cornea is increased in flatter lens fits, 
potentially leading to peripheral corneal desiccation. Flat lens fits could also lead to an altered eyeblink frequency that may arise from an increase in interaction between the upper eyelid and the edge of the lens.

In relation to corneal coverage, larger lenses could possibly increase the attraction of tears from the ocular surface due to their greater circumference and the consequent increased length of the tear meniscus, thus supporting the tear volume theory. The effect of lens size on the bridging effect and thus on mucin spreading is uncertain. On one hand larger lenses may cover more of the affected 3- and 9o'clock positions on the cornea, which could prevent exposure of these areas of the cornea. On the other hand, smaller lenses leave more of the cornea exposed to the upper eyelid during a blink, facilitating better mucin spreading over the corneal periphery. Also, small lenses may have smaller edge clearances thus decreasing the bridge effect. Therefore the effect of lens size with regard to the bridging effect is uncertain. The same applies to the effect of lens size on eyeblinking, and these factors may or may not be inter-related. Lens size is directly related to the type of lens fit since larger lenses are more likely to result in lid attachment fits and smaller lenses to interpalpebral lens fits. ${ }^{24,28,29}$

The finding that more 3-and 9-o'clock staining was seen in interpalpebral lens fits than in lid attachment fits seems to point directly at the eyeblink inhibition theory, and is less likely to support the other two theories relating to tear volume and the bridging effect. It may be noted that lid attachment lens fits could be associated with more lens movement, but no difference in lens movement was found between the two types of lens fits in this study.

No evidence was found in our study to support the mechanical theory in which with-the-rule corneal toricity is considered as a risk factor. No correlation between the amount of corneal staining and amount or type of corneal astigmatism was found. However, because this was established by looking at central keratometry only, this finding should not be considered conclusive, as discussed above.

In summary, the results of this study seem to point to more than one possible etiology for 3- and 9-o'clock staining, but the tear volume theory and the eyeblink inhibition theory appear to be most widely supported. The bridging effect is another possibility, although this is supported directly by the flat lens fit result only. No evidence in our study was found to support the mechanical theory. 


\section{Conclusions}

The results of this study indicate that flat fitting lenses, interpalpebral lens fits and larger lenses increase the risk for development of 3- and 9-o'clock staining. These variables cannot be seen as isolated factors, and controversy remains as to strategies to avoid 3-and 9-o'clock staining. Avoiding flat lens fits as such seems beneficial to remedy 3- and 9-o'clock staining, but flat fitted lenses can also create a lid attachment type of fit that could in turn be beneficial. The same was found for corneal coverage. Less 3- and 9-o'clock staining was seen with smaller lenses in this study, but larger lenses can lead to a lid attachment fit that can be beneficial in the management of the condition.

The results of this study lend support to more than one possible etiology for 3-and 9-o'clock staining. The two most widely supported theories appear to be the tear volume theory and the eyeblink inhibition theory. Further research, especially focusing on tear volume and on eyeblink behavior in GP lens wear, is needed to interpret these findings in more detail.

\section{Acknowledgements}

The authors wish to thank Jantine Bos and Rob Faas of Groenhof Optiek in Amstelveen, the Netherlands, and Peter van Etten for their help during the study. Thanks are also due to Ineke Krijger and Matthew Lampa for their comments on this paper. 


\section{References}

1. Van der Worp E, De Brabander J, Swarbrick H, Nuijts R, Hendrikse F. Corneal desiccation in rigid contact lens wear: 3- and 9-o'clock staining. Optom Vis Sci 2003;80:280-90.

2. Steele E. Observations on the fitting of corneal contact lenses. Am J Optom Arch Am Acad Optom 1959;36:194-9.

3. Barabas R, Fontana A. Juxtaposition staining. Contacto 1967;11:3-6.

4. Graham R. Persistent nasal and temporal stippling. Contacto 1968;12:20-12.

5. Solomon J. Causes and treatments of peripheral corneal desiccation. Contact Lens Forum 1986;11:30-6.

6. Ghormley N, Bennett E, Schnider C. Corneal desiccation-clinical management. Int Contact Lens Clin 1990;17:5.

7. Holden T, Bahr K, Koers D, Edrington T. The effect of secondary curve lift-off on peripheral corneal desiccation. Am J Optom Physiol Opt 1987;64:313-20.

8. Buch J. Peripheral corneal staining: a survey of RGP laboratories. Contact Lens Spectrum 1997;12(7):31-7.

9. Schnider C, Terry R, Holden B. Effect of lens design on peripheral corneal desiccation. J Am Optom Assoc 1997;68:163-70.

10. Jones D, Bennett E, Davis L. How to manage peripheral corneal desiccation. Contact Lens Spectrum 1989;4(5):63-6.

11. Lowther G. Review of rigid contact lens design and effects of design on lens fit. Int Contact Lens Clin 1988;15:378-89.

12. Lebow K. Reduce three-and-nine corneal staining with moderate edge lift profiles. Contact Lens Spectrum 1990;5(7):20-33.

13. Van der Worp E, De Brabander J, Swarbrick H, Hendrikse F. Eyeblink frequency and type in relation to 3- and 9-o'clock staining and gas permeable contact lens variables Optom Vis Sci 2008;85:857-66

14. Efron N. Grading scales for contact lens complications. Ophthalmic Physiol Opt 1998;18:1826.

15. Terry RL, Schnider CM, Holden BA et al. CCLRU standards for success of daily and extended wear contact lenses. Optom Vis Sci 1993;70:234-43.

16. Businger U, Treiber A, Flury $C$. The etiology and management of three and nine o'clock staining. Int Contact Lens Clin 1989;16:136-9.

17. Jones L, Jones D. Common Contact Lens Complications. Oxford: Butterworth-Heinemann, 2000:70-3.

18. Guillon JP. Use of the Tearscope Plus and attachments in the routine examination of the marginal dry eye contact lens patient. Adv Exp Med Biol 1998;438:859-67.

19. Lowther GE. Dryness, Tears, and Contact Lens Wear: Clinical Practice in Contact Lenses. Boston: Butterworth-Heinemann, 1997:84-90.

20. Jones L, Woods CA, Efron N. Life expectancy of rigid gas permeable and high water content contact lenses. CLAO J 1996;22:258-61.

21. Woods CA, Efron N. Regular replacement of extended wear rigid gas permeable contact lenses. CLAO J 1996;22:172-8.

22. Hulley SB, Cummings SR, Browner WS, Grady D, Hearst N, Newman TB. Designing Clinical Research. Philadelphia: Lippincott Williams \& Wilkins, 2001:65-91.

23. Morgan $\mathrm{P}$, Efron $\mathrm{N}$, Woods $\mathrm{C}$ et al. International contact lens prescribing 2007. Contact Lens Spectrum 2008;23(1):36-41.

24. Williams CE. New design concepts for permeable rigid contact lenses. J Am Optom Assoc 1979;50:331-6. 
25. Brannen R. Incidence of central edema and peripheral staining as a function of contact lens fitting philosophy. J Am Optom Assoc 1977;48:391-5.

26. Grohe R, Lebow K. Vascularized limbal keratitis. Int Contact Lens Clin 1989;16:197-209.

27. Barr J, Testa L. Corneal epithelium 3 and 9 o'clock staining studied with the specular microscope. Int Contact Lens Clin 1994;21:105-11.

28. Korb D, Korb J. A new concept in contact lens design; parts 1 \& 2. J Am Optom Assoc 1970;41:1-12.

29. Andrasko G. Peripheral corneal staining: Edge lift \& extended wear. Contact Lens Spectrum 1990;5(8):33-5.

30. Barr J. Aspheric update. Contact Lens Spectrum 1988;3(11):56-62.

31. De Brabander J, Brinkman C, Kok J, Van Mil C. Low or High Dk? Spherical or aspherical rigid gas permeable lenses? The gap between theory and practice. JBCLA 1992;15(4):169-2.

32. Franklin RJ, Morelande MR, Iskander DR, Collins MJ, Davis BA. Combining central and peripheral videokeratoscope maps to investigate total corneal topography. Eye Contact Lens 2006;32:27-32.

33. Read SA, Collins MJ, Carney LG, Franklin RJ. The topography of the central and peripheral cornea. Invest Ophthalmol Vis Sci 2006;47:1404-15.

34. Schnider C, Terry R, Holden B. Effect of patient and lens performance characteristics on peripheral corneal desiccation. J Am Optom Assoc 1996;67:144-50.

35. Lowther G. Peripheral corneal staining - can it be prevented? Int Contact Lens Clin 1989;16:37.

36. Efron N. Blinking. In: Efron N, ed. Contact Lens Complications. Oxford: ButterworthHeinemann, 1999:3-9.

37. Douthwaite WA. Contact Lens Optics and Lens Design. Bradford: Butterworth-Heinemann, 2006:69-80.

38. Efron N. Staining. In: Efron N, ed. Contact Lens Complications. Oxford: ButterworthHeinemann, 1999:75-81.

39. Lebow K. Peripheral corneal staining. In: Silbert J, ed. Anterior Segment Complications of Contact Lens Wear. Edinburgh: Churchill Livingstone, 1994:59-90.

40. Tomlinson A. Contact lens-induced dry eye. In: Tomlinson A, ed. Complications in Contact Lens Wear. St. Louis: Mosby, 1992:195-218.

41. Begley CG, Chalmers RL, Abetz L et al. The relationship between habitual patient-reported symptoms and clinical signs among patients with dry eye of varying severity. Invest Ophthalmol Vis Sci 2003;44:4753-61.

42. Holly F. Tear film physiology in contact lens wear, Part II, Contact lens tear film interactions. Am J Optom Physiol Opt 1981;58:331-141.

43. Brubaker RF, Ezekiel S, Chin L, Young L, Johnson SA, Beeler GW. The stress-strain behavior of the corneoscleral envelope of the eye. I. Development of a system for making in vivo measurements using optical interferometry. Exp Eye Res 1975;21:37-46.

44. McDonald JE, Brubaker S. Meniscus-induced thinning of tear films. Am J Ophthalmol 1971;72:139-46.

45. Bennett E, Egan D. Rigid gas permeable lens problem solving. J Am Optom Assoc 1986;57:504-11.

46. Fonn D. Preventing contact lens dropouts. Contact Lens Spectrum 2002;17:43-51. 



\section{CHAPTER 5}

Tear Volume and Eyeblinking in Relation to 3- and 9-O'Clock Staining

"I shut my eyes - in order to see". (Paul Gauguin) 


\section{Introduction}

In this chapter, possible mechanisms behind the development of 3-and 9-o'clock staining are analysed. Part 5.1 describes a study comparing tear meniscus height (as a representative of tear volume) in rigid gas permeable lens wearers versus non-lens wearers, and comparing tear meniscus height in subjects with substantial 3- and 9o'clock staining to those without. Part 5.2 looks at the quality and quantity of eyeblinks in rigid gas permeable lens wear. It investigates the possible difference in frequency and in completeness of eyeblinks in non-lens wearers and in rigid gas permeable lens wearers (with and without 3-and 9-o'clock staining). 


\section{1 \\ Tear Meniscus Height Evaluation in Rigid Gas Permeable Lens Wear}

Van der Worp E, de Brabander J, Swarbrick H, Hendrikse F.

Eye Research Institute Maastricht, Department of Ophthalmology Academic Hospital, Maastricht University, The Netherlands (EW, JB, FH) and the School of Optometry and Vision Science, University of New South Wales, Sydney, Australia (HS) 


\begin{abstract}
Purpose. The aim of this study is to investigate if differences in tear meniscus height (TMH) exist between rigid gas permeable (GP) lens wearers and non-lens wearers, and furthermore if differences exist between subjects with substantial 3and 9-o'clock staining and those without. In addition, the possible relationship between lens fit and TMH is investigated.
\end{abstract}

Methods. The study involved 26 non-lens wearers and 55 rigid gas permeable (GP) contact lens wearers, with and without 3- and 9-o'clock staining. A video recording of the tear meniscus on the lower eyelid was generated with a CCD camera and a DVD recorder to measure the TMH from a computer screen. Subjects were categorized as either having < grade 2 staining (low staining group) or $\geq$ grade 2 staining (high staining group) based on a specially designed image grading scale for 3- and 9-o'clock staining.

Results. The TMH in GP lens wearers was $0.20 \pm 0.08 \mathrm{~mm}$, while it was $0.28 \pm$ $0.10 \mathrm{~mm}$ in non-lens wearers $(\mathrm{p}<0.01)$. Within the lens wearing group, the TMH in the high staining group was $0.19 \pm 0.09 \mathrm{~mm}$, while it was $0.21 \pm 0.08$ in the low staining group, but this difference was not statistically significant. No statistically significant differences in TMH in relation to lens fit variables were found (with the exception of hyperopic lens corrections).

Conclusions. The finding that TMH values in GP lens wearers were found to be lower than in non-lens wearers seems to support the hypothesis that GP lenses withhold tear volume from the anterior ocular surface. In general, no relationship between TMH and contact lens fit related variables were found, and no statistically significant difference in TMH between the low staining group and the high staining group was found: either there is no relationship between these variables, or the method of measuring TMH is not sensitive enough to determine potentially small differences in $\mathrm{TMH}$.

Key words: GP contact lenses, tear volume, tear meniscus height, 3- and 9-o'clock corneal staining, lens fit 
The total amount of tears present on the ocular surface within the palpebral aperture has been estimated at between $7 \mu \mathrm{l}$ and $10 \mu \mathrm{l} .{ }^{1}$ Any fluid amount above $10 \mu \mathrm{l}$ is thought to be flushed out of the normal palpebral fissure, although this will vary with the size of the palpebral fissure. ${ }^{1}$ Of the total volume of tear fluid present within the palpebral aperture, about $70-90 \%$ is thought to be consumed by the tear menisci lining the upper and the lower eyelid margins. ${ }^{2-4}$

When a rigid gas permeable (GP) contact lens is placed on the ocular surface, another tear meniscus is added that surrounds the entire lens edge. According to Holly $^{5}$ and others ${ }^{6,7}$ this could be one of the underlying causes of 3- and 9-o'clock staining: capillary attraction of tears to the tear meniscus adjacent to the lens edge could attract substantial amounts of fluids. The tear film in the area immediately adjacent to the tear meniscus at the lens edge may become thin and can break up easily, causing a dry area and subsequent staining. In theory: the thicker the lens edge and the higher the edge clearance, the greater the volume of tears attracted to this area. ${ }^{8}$

The tear meniscus height (TMH) was measured and analyzed as an indication of the total tear volume available on the ocular surface to evaluate the tear volume mechanism and the possible development of 3- and 9-o'clock staining in relation to that.

The aim of this study is to investigate whether differences in lower eyelid TMH exist between GP lens wearers and non-lens wearers, and furthermore if differences exist between subjects with substantial 3- and 9-o'clock staining and those without. In addition, the possible influence of lens fit on $\mathrm{TMH}$ is investigated.

\section{Methods}

\section{Subjects and Lens Wear}

This study involved 81 subjects. Of these, 55 were GP contact lens wearers (110 eyes), both with and without 3-and 9-o'clock staining, from the database of a large contact lens practice in the Netherlands. The control group consisted of 26 randomly selected non-lens wearing patients from the same practice. The GP lens wearers included in this study were long term, successful contact lens wearers who were seen as part of a regular checkup schedule.

Information about the ocular history was obtained by interviewing the subjects. All tested eyes were free of pathological eye conditions, including pathological dry eye conditions. No additional dry eye testing was performed. Subjects were asked to 
wear their lenses for a minimum of one hour on the day of the examination visit. The measurements took place between $9 \mathrm{am}$ and $6 \mathrm{pm}$. All procedures in this study were conducted in accordance with the Declaration of Helsinki (2000), and all subjects signed an informed consent form prior to participation in the study.

\section{Corneal Staining}

A detailed slitlamp (Nikon FS-3) examination was performed using fluorescein (Fluorets, Chauvin), cobalt blue light and a Wratten \#12 yellow barrier filter.

A specially designed image grading scale for 3- and 9-o'clock staining, developed at the University of Maastricht, ${ }^{9}$ was used to grade all eyes in decimals on a $0-4$ point scale. Typically, grade 2 on $0-4$ clinical grading scales is accepted as a critical clinical cut-off point for the management of contact lens related conditions. ${ }^{10,11} \mathrm{Up}$ to grade 2 is considered to be within normal limits, wheras grade 2 or higher usually requires clinical action. In this study, subjects were categorized as either having < grade 2 staining or $\geq$ grade 2 staining, referred to as the 'low' and 'high' staining group respectively, using the developed grading scale.

\section{TMH Measurement}

A video recording of the lower TMH with a 40x slitlamp magnification was generated with a CCD camera (Mitsubishi CCD-100E) and a DVD recorder (Panasonic DMR E30). This allowed the investigators to freeze the image and measure the TMH from the obtained image. The DVD generated image was further magnified on a 17 inch computer monitor. The TMH was then measured as the distance between the darker edge of the lower eyelid and the top of the reflex from the tear strip. ${ }^{12} \mathrm{~A}$ time-averaged assessment was made by taking three TMH measurements over the recorded time at the mid-position of the lower eyelid. ${ }^{13}$ Each time the image was frozen, enlarged and TMH measurement was taken directly from the computer screen. The average of three measurements was taken and used for further analysis.

To investigate the relationship between TMH and the lens variables, and for the relationship between TMH and 3- and 9-o'clock staining, the TMH of 110 eyes was used for analysis. For TMH in GP lens wear versus non-lens wear, the number of subjects $(\mathrm{n}=81)$ was used and the average TMH for the left and right eye was used for analysis.

\section{Lens Variables}

To analyze lens fit, the tear layer thickness was assessed from the DVD recordings by using fluorescein and the same slitlamp technique as described with corneal staining. The lens was held in a static, central position on the cornea and both the 
horizontal and vertical direction of the lens fit were analyzed. The tear layer thickness was graded on a five point scale as either optimal (0), slightly too thick/thin but acceptable (suboptimal, grade 1) or excessive (grade 2). The tear layer thicknesses centrally, mid-peripherally and at the edge of the lens were combined and converted into three categories of lens fit. Lens fits were categorized as a flat fit if an unacceptably thin tear layer $(-2)$ was seen centrally and/or an unacceptably thick $(+2)$ tear layer was seen in the midperiphery or at the edge of the lens. An opti$\mathrm{mal} /$ acceptable lens fit was characterized by a tear layer thickness of grade $0,+1$ or 1 in any location. Steep lens fits showed an unacceptably thick tear layer $(+2)$ centrally and/or an unacceptably thin (-2) tear layer in the midperiphery or at the edge of the lens.

Lens size was subjectively rated on-eye as optimal, large or small, and is referred to as corneal coverage. An 11:9 corneal coverage ratio between the vertical visible iris diameter and total lens diameter was considered optimal. Corneal coverage was the preferred method (as opposed to analysis of absolute lens diameter) because it best reflects whether the size of the lens is appropriate for a particular cornea.

The effect of edge shape is very complex to analyze, especially in a clinical setting. To a lesser extent the same is true for edge thickness. Edge shape and edge thickness were not analyzed in this study due to the large variation of possibilities and the limitations with regard to evaluating this. Instead, the spherical equivalent of the refraction (average of the two eyes) was analyzed in relation to 3- and 9-o'clock staining.

To assess the position of the lens in relation to the upper eyelid, all lens fits were rated as either interpalpebral (the contact lens is positioned between the eyelids during interblink periods) or lid attachment (the lens stays under the upper eyelid in between eyeblinks).

\section{Data Analysis}

Statistical analysis of the data was performed using Statistical Package for Social Sciences (SPSS) version 11.5. The data were tested for differences in TMH between different groups using the independent Student's t-test and ANOVA test. Power analysis was used to ensure an adequate sample size. ${ }^{14} \mathrm{~A}$ critical $\mathrm{p}$ value of 0.05 was used to denote statistical significance for all analysis. Correlations between variables were analyzed by calculating Spearman's rho because the distribution of data regarding wearing time and spherical equivalent refraction was non-Gaussian in nature. All data are presented as mean and standard deviation, unless otherwise stated. 


\section{Results}

The low staining group comprised 85 eyes $(77 \%)$ of lens wearers, while the high staining group consisted of 25 eyes (23\%). The mean age of subjects in the nonlens wearing group was $33.8 \pm 10.9$, in the low staining group this was $42.7 \pm 14.2$ years and $43.0 \pm 11.1$ years in the high stain group. A statistical significant difference in age was found between the non-lens wearing group and the lens wearing group $(\mathrm{p}<0.01)$, but no difference in age between the low and the high staining group was found. The proportion of male to female subjects was $27 \%$ male versus $73 \%$ female, which is a realistic reflection of the gender ratio of lens fits around the world. ${ }^{15}$ No statistically significant difference for gender was found.

The subjects had been wearing GP lenses for an average of $19.9 \pm 10.6$ years (range 1.5 to 38 years). The median wearing time of the lenses on the day of examination was 3.5 (1.0 to 11.0) hours. The hours of lens wear prior to examination in the high staining grouping (mean $5.9 \pm 3.1$ hours) was higher than in the low $(3.9 \pm$ 3.0 hours) staining group ( $\mathrm{t}$-test, $\mathrm{p}=0.01$ ).

The TMH in GP lens wearers was $0.20 \pm 0.08 \mathrm{~mm}$, while it was $0.28 \pm 0.10 \mathrm{~mm}$ in non-lens wearers (table 1). A statistically significant difference in $\mathrm{TMH}$ was found for these two groups $(\mathrm{p}<0.01)$.

TABLE 1. Summary of TMH values $(\mathrm{mm})$ for different variables with their statistical significance

\begin{tabular}{llll}
\hline TMH values $(\mathrm{mm})$ & & $\mathrm{p}$ value \\
\hline $\begin{array}{l}\text { GP lens wearers }(\mathrm{n}=26) \\
0.20 \pm 0.08\end{array}$ & $\begin{array}{l}\text { Non-lens wearers }(\mathrm{n}=55) \\
0.28 \pm 0.10\end{array}$ & $\mathrm{p}<0.01$ \\
Low stain group $(\mathrm{n}=85)$ & High stain group $(\mathrm{n}=25)$ & & \\
$0.19 \pm 0.09$ & $0.21 \pm 0.08$ & & $\mathrm{p}=0.41$ \\
Steep $(\mathrm{n}=7)$ & Optimal/Suboptimal $(\mathrm{n}=-64)$ & Flat $(\mathrm{n}=39)$ lens fit & \\
$0.20 \pm 0.06$ & $0.20 \pm 0.08$ & $0.20 \pm 0.08$ & $\mathrm{p}=0.99$ \\
Lid attachment $(\mathrm{n}=81)$ & Interpalpebral lens fit $(\mathrm{n}=29)$ & & \\
$0.19 \pm 0.07$ & $0.23 \pm 0.09$ & & $\mathrm{p}=0.06$ \\
Large $(\mathrm{n}=15)$ & Optimal $(\mathrm{n}=79)$ & Small rated lenses $(\mathrm{n}=16)$ & \\
$0.19 \pm 0.07$ & $0.20 \pm 0.08$ & $0.21 \pm 0.07$ & $\mathrm{p}=0.93$ \\
\hline
\end{tabular}

Within the lens wearing group, the TMH in the high staining group was $0.19 \pm$ $0.09 \mathrm{~mm}$ while it was $0.21 \pm 0.08 \mathrm{~mm}$ in the low staining group, but this difference was not statistically significant. 
Looking at lens fit: no differences in TMH were found. The TMH in steep lens fits was $0.20 \pm 0.06 \mathrm{~mm}$ while it was $0.20 \pm 0.08 \mathrm{~mm}$ for optimal and suboptimal lens fits and for flat lens fits it also was found to be $0.20 \pm 0.08 \mathrm{~mm}$.

Higher TMH values were seen in interpalpebral fits than in lid attachment lens fits, respectively $0.23 \pm 0.09 \mathrm{~mm}$ and $0.19 \pm 0.07 \mathrm{~mm}$, but this difference was not statistically significant.

Corneal coverage revealed a slightly lower mean TMH for large rated lenses $(0.19 \pm$ $0.07 \mathrm{~mm})$ compared to optimal rated lenses $(0.20 \pm 0.08 \mathrm{~mm})$ and small rated lenses $(0.21 \pm 0.07 \mathrm{~mm})$, but no statistically significant difference was found.

The spherical equivalent refraction ranged from $-0.50 \mathrm{D}$ to $-10.00 \mathrm{D}$ in myopic subjects (median -3.13D, $\mathrm{n}=97$ ) and from +2.25D to +7.00D (median +4.00D, $\mathrm{n}=13$ ) in hyperopic subjects. The correlation between TMH and overall spherical equivalent of the refraction was low $(\mathrm{r}=-0.117, \mathrm{p}=0.23)$, for myopic correction the correlation was $\mathrm{r}=-0.137 \quad(\mathrm{p}=0.18)$ while for hyperopic corrections this was $\mathrm{r}=-0.583$ $(\mathrm{p}=0.04)$.

\section{Discussion}

The TMH in GP lens wearers was found to be lower than in non-lens wearers. This unique finding seems to support the theory that GP lenses attract tears to the area underneath and/or next to the periphery of the lens, leaving the rest of the exposed ocular surface with a thinner tear layer and shorter tear break-up-times. It should be kept in mind that the age of subjects in the non-lens wearing group was lower than that of subject in the lens wearing group, and age may be a contributing factor to this finding. Ainally, it must be mentioned that the amount of staining that is observed in subjects with dry eye may be related to the time of day they are seen. ${ }^{16}$ Our results show a statistically significant but low correlation between the level of corneal staining and hours of lens wear prior to examination. There was also a statistically significant difference in hours of lens wear prior to examination between the high and the low staining group. This leaves open the possibility that subjects in the low staining group may have progressed to the high staining group levels if they had been seen later in the day or after a longer period of lens wear. In this study subjects were seen in a private practice, often as part of a regular check up. Some patients were not able or willing to adjust for longer wearing times.

A TMH of less than $0.30 \mathrm{~mm}$ has been mentioned by some to be an indication of a dry eye in clinical settings. ${ }^{1,17,18}$ This would mean that the TMH in GP lens wear- 
ers found in this study $(0.20 \mathrm{~mm})$ would be considered as too minute. However, also the non-lens wearing control group did not fully comply with the criteria (a TMH of $0.28 \mathrm{~mm}$ was found in non-lens wearers). The TMH values found in this study are within the same range as most of the reported values in previous studies. ${ }^{12,19-22}$ But large variations between studies exist in TMH outcome. Some have found significantly lower TMH values in normal subjects, and even suggested that values of $>0.25 \mathrm{~mm}$ in normal individuals may indicate slight reflex tearing. ${ }^{12}$ The purpose of this study was to evaluate potential differences in TMH between GP lens wearers and non-lens wearers, rather than attempting to define absolute TMH values for specific groups of subjects.

Theoretically, the lower TMH values in GP lens wearers than in non-lens wearers can be caused by capillary attraction of tears in the corneal area adjacent to the lens edge. Due to this capillary attraction, the tear film in the area immediately adjacent to the lens edge may become very thin and can break up easily, causing a dry area and consequently 3- and 9-o'clock staining (the tear volume theory). ${ }^{5}$ But GP lens wearers in the high staining group did not show a statistically significant difference in TMH with subjects in the low staining group, despite the fact that a small difference in $\mathrm{TMH}$ was found in absolute terms.

In previous studies, flat lens fits, large diameter lenses and interpalpebral lens fits were found to be possible risk factors for the development of 3- and 9-o'clock staining, which seems to be in line with the tear volume theory. But no relationship between $\mathrm{TMH}$ and any of these variables was found in this study.

The TMH was similar in flat lens fits, optimal/suboptimal lens fits and steep lens fits, where it may have been expected that flat lenses cause lower TMH values due to the increase in tear volume attraction by the higher lens edge clearance.

Theoretically, larger lenses may be expected to attract a higher volume of tears to the area underneath the lens, leaving a lower amount of tear volume available on the ocular surface. Corneal coverage in this study showed slightly lower TMH values for large rated lenses compared to optimal and small rated lenses, but this difference was not found to be statistically significant.

Higher TMH values in interpalpebral fits lens were seen than in lid attachment lens fits, although this difference was not found to be statistically significant. It is subject to speculation why the TMH was found to be higher in interpalpebral fits. Because interpalpebral lens fits are being considered a risk factor for 3- and 9o'clock staining, the opposite may have been expected. Possibly, the upper eyelid 
caused pressure on the superior part of the lens in lid attachment fits, causing a lift in the inferior part of the lens away from the cornea. This may have subtracted fluids from the inferior ocular surface, causing a decrease in tear volume in the inferior part of the ocular surface and lower TMH values on the lower eyelid.

Myopic lenses in this study showed no correlation with TMH, while hyperopic lenses showed a higher and significant correlation with TMH. Higher myopic correction generally is thought to be associated with a thicker lens edge, and with a higher hyperopic correction typically a thinner lens edge is expected. ${ }^{23}$ This effect may be limited due to the fact that high minus lenses often utilize a lenticular edge design to reduce the lens edge thickness. However, generally the periphery of high minus lenses still is considered to be thicker than that of hyperopic lenses. In summary, there may be a possible relationship between edge thickness and spherical equivalent of the refraction, but the exact nature of this relationship is unclear, and based on the findings of this study no statement can be made regarding edge thickness in relation to $\mathrm{TMH}$.

One possible factor that may affect the outcome of this study is the method used to measure tear volume. Measuring tear volume in clinical practice is not an easy task. $\mathrm{TMH}$ measurement on the lower eyelid has been proposed by many investigators to obtain an indication of tear volume on the ocular surface. ${ }^{13,24-28}$ A scanty appearance of the lower tear meniscus or local areas of discontinuity are believed to be signs of a tear film deficiency. TMH measurements have been found to correlate well with results of the phenol red test and tear stability tests. ${ }^{27,29}$ Observation and measurement of the TMH and the regularity of the tear meniscus has been suggested as a simple and fast alternative to get an indication of the available tear volume on the ocular surface, ${ }^{1}$ also during contact lens wear. ${ }^{30}$ But although currently available tear volume tests such as the Schirmer test, phenol red test and TMH measurement are able to reveal pathological dry eye situations, it has been pointed out that there is a high variability in outcome and it is unclear whether these tests are able to pick up minor aqueous deficiencies. Especially small changes to tear volume on the anterior eye that may occur during, and possibly as the result of, contact lens wear may be difficult to measure. One should keep in mind that the standard deviation of the TMH found in this study was $0.09 \mathrm{~mm}$, where the mean differences between groups of contact lens related variables were often not larger than $0.02 \mathrm{~mm}$.

It should also be kept in mind that analysis of lens variables was sometimes different in the right and left eye of the same subject while tear production may be an overall process involving both eyes. This makes the analysis of TMH in relation to 
lens variable a complicated task. Future, prospective studies aiming at controlling for this as much as possible would be needed to analyze lens variables in relation to TMH in more detail.

Also, Shen et al. ${ }^{31}$ recently found a difference in TMH shortly after eye opening, compared to the baseline before sleep at $10 \mathrm{pm}$. All measurements in our study were taken between 9am and $6 \mathrm{pm}$, but a statistically significant difference in hours of lens wear prior to examination between the high and the low staining group was found, assuming a different time of day when measurements took place. Also, it must be mentioned that the difference in wearing time leaves open the possibility that subjects in the low staining group may have progressed to the high staining group levels if they had been seen later in the day or after a longer period of lens wear. In this study subjects were seen in a private practice, often as part of a regular check up. Some patients were not able or willing to adjust for longer wearing times. Future studies should control better for time of measurement, excluding this as a possible factor.

More recently, investigators ${ }^{21,31-34}$ have reported on using ocular coherence tomography as a possible tool to measure TMH. This method may possibly be a more accurate method of measuring tear meniscus height in the future than the manual measurement technique from slitlamp images that was used in our study.

\section{Conclusions}

TMH values in GP lens wearers were found to be lower than in non-lens wearers. This finding seems to support the hypothesis that GP lenses withhold tear volume from the anterior ocular surface. However, no statistically significant differences in TMH between the low staining group and the high staining group were found. In general, contact lens related variables showed no relationship with TMH. Either there are no substantial differences in TMH or the method of investigating this is not sensitive enough to pick up the subtle differences that may occur.

\section{Acknowledgements}

Many thanks to Jantine Bos and Rob Faas of Groenhof Optiek in Amstelveen, the Netherlands and to Peter van Etten for his help during the study. Special thanks Ineke Krijger and Matthew Lampa for their valuable comments on this paper. 


\section{References}

1. Korb D, Craig J, Doughty M, Guillon J-P, Smith G, Tomlinson A. The Tear Film: structure, function and clinical examination. London: Butterworth-Heinemann, 2002:51-83.

2. Mishima S, Gasset A, Klyce SD, Jr., Baum JL. Determination of tear volume and tear flow. Invest Ophthalmol 1966;5:264-76.

3. Kwok LS. Calculation and application of the anterior surface area of a model human cornea. J Theor Biol 1984;108:295-313.

4. Korb D, Craig J, Doughty M, Guillon J-P, Smith G, Tomlinson A. The Tear Film: structure, function and clinical examination. London: Butterworth-Heinemann, 2002:18-51.

5. Holly F. Tear film physiology in contact lens wear, Part II, Contact lens tear film interactions. Am J Optom Physiol Opt 1981;58:331-141.

6. Brubaker RF, Ezekiel S, Chin L, Young L, Johnson SA, Beeler GW. The stress-strain behavior of the corneoscleral envelope of the eye. I. Development of a system for making in vivo measurements using optical interferometry. Exp Eye Res 1975;21:37-46.

7. McDonald JE, Brubaker S. Meniscus-induced thinning of tear films. Am J Ophthalmol 1971;72:139-46.

8. Van der Worp E, De Brabander J, Swarbrick H, Nuijts R, Hendrikse F. Corneal desiccation in rigid contact lens wear: 3- and 9-o'clock staining. Optom Vis Sci 2003;80:280-90.

9. Van der Worp E, De Brabander J, Swarbrick H, Hendrikse F. Eyeblink frequency and type in relation to 3- and 9-o'clock staining and gas permeable contact lens variables Optom Vis Sci 2008;85:857-66

10. Efron N. Grading scales for contact lens complications. Ophthalmic Physiol Opt 1998;18:1826.

11. Terry RL, Schnider CM, Holden BA et al. CCLRU standards for success of daily and extended wear contact lenses. Optom Vis Sci 1993;70:234-43.

12. Doughty MJ, Laiquzzaman M, Oblak E, Button N. The tear (lacrimal) meniscus height in human eyes: a useful clinical measure or an unusable variable sign? Cont Lens Anterior Eye 2002;25:57-65.

13. Doughty MJ, Laiquzzaman M, Button NF. Video-assessment of tear meniscus height in elderly Caucasians and its relationship to the exposed ocular surface. Curr Eye Res 2001;22:420-6.

14. Hulley SB, Cummings SR, Browner WS, Grady D, Hearst N, Newman TB. Designing Clinical Research. Philadelphia: Lippincott Williams \& Wilkins, 2001:65-91.

15. Morgan P, Efron N, Woods C et al. International contact lens prescribing 2007. Contact Lens Spectrum 2008;23(1):36-41.

16. Begley CG, Chalmers RL, Abetz L et al. The relationship between habitual patient-reported symptoms and clinical signs among patients with dry eye of varying severity. Invest Ophthalmol Vis Sci 2003;44:4753-61.

17. Lowther GE. Dryness, Tears, and Contact Lens Wear: Clinical Practice in Contact Lenses. Boston: Butterworth-Heinemann, 1997:84-90.

18. Terry JE. Eye disease of the elderly. J Am Optom Assoc 1984;55:23-9.

19. Johnson ME, Murphy PJ. The agreement and repeatability of tear meniscus height measurement methods. Optom Vis Sci 2005;82:1030-7.

20. Lemp MA. Report of the National Eye Institute/Industry workshop on Clinical Trials in Dry Eyes. CLAO J 1995;21:221-32.

21. Bitton E, Keech A, Simpson T, Jones L. Variability of the analysis of the tear meniscus height by optical coherence tomography. Optom Vis Sci 2007;84:903-8.

22. Purslow C, Wolffsohn J. The relation between physical properties of the anterior eye and ocular surface temperature. Optom Vis Sci 2007;84:197-201. 
23. Douthwaite WA. Contact Lens Optics and Lens Design. Bradford: Butterworth-Heinemann, 2006:69-80.

24. Klein M. The lacrimal strip and the precorneal flim in cases of Sjögren's syndrome. Br J Ophthalmol 1949;33:387-8.

25. Wright P. Diagnosis and management of dry eyes. Trans Ophthalmol Soc UK 1971;91:119-28.

26. Baum JL. Systemic disease associated with tear deficiencies. Int Ophthalmol Clin 1973;13:15784.

27. Mainstone JC, Bruce AS, Golding TR. Tear meniscus measurement in the diagnosis of dry eye. Curr Eye Res 1996;15:653-61.

28. Holly FJ, Lemp MA. Tear physiology and dry eyes. Surv Ophthalmol 1977;22:69-87.

29. Golding TR, Bruce AS, Mainstone JC. Relationship between tear-meniscus parameters and tearfilm breakup. Cornea 1997;16:649-61.

30. Efron N. Tear film dysfunction. In: Efron N, ed. Contact Lens Complications. Oxford: Butterworth-Heinemann, 1999:61-71.

31. Shen M, Wang J, Tao A et al. Diurnal variation of upper and lower tear menisci. Am J Ophthalmol 2008;145:801-6 e2.

32. Wang J, Palakuru JR, Aquavella JV. Correlations Among Upper and Lower Tear Menisci, Noninvasive Tear Break-up Time, and the Schirmer Test. Am J Ophthalmol 2008;145:795-800 e1.

33. Palakuru JR, Wang J, Aquavella JV. Effect of blinking on tear dynamics. Invest Ophthalmol Vis Sci 2007; 48:3032-7.

34. El Maftouhi A, Baudouin C. [Tear meniscus in Visante OCT]. J Fr Ophtalmol 2007;30:559. 


\section{2 \\ Eyeblink Frequency and Type in Relation to 3- and 9-O'Clock Staining and Gas Permeable Contact Lens Variables}

Van der Worp E, de Brabander J, Swarbrick H, Hendrikse F.

Optometry \& Vision Science 2008;85:857-66

Eye Research Institute Maastricht, Department of Ophthalmology Academic Hospital, Maastricht University, The Netherlands (EW, JB, FH) and the School of Optometry and Vision Science, University of New South Wales, Sydney, Australia (HS) 


\section{Abstract}

Purpose. The primary goal of this study was to evaluate if there is a difference in frequency and in completeness of eyeblinks (type of eyeblink) in rigid gas permeable (GP) lens wearers experiencing 3- and 9-o'clock staining compared to GP lens wearers without substantial staining and non-lens wearers.

Methods. The study involved 26 non-lens wearers and 55 GP contact lens wearers, with and without 3-and 9-o'clock staining. Every eyeblink over a 5-minute period was categorized as a complete eyeblink, incomplete eyeblink or an eyeblink attempt. Additionally, type of lens fit (interpalpebral versus lid attachment), lens-tocornea fit (flat versus steep), spherical equivalent of the refraction and corneal coverage (on-eye lens diameter) were analyzed.

Results. No difference in overall eyeblink frequency was found between non-lens wearers (group I, $n=26$ ), GP lens wearers with less than grade 1 corneal staining (group II, $\mathrm{n}=25$ ) and GP lens wearers with grade 1 or more corneal staining (group III, $\mathrm{n}=30$ ). Fewer complete eyeblinks, more incomplete eyeblinks and more eyeblink attempts were seen in group III than in group I or II $(\mathrm{p}<0.01, \mathrm{p}=0.03$, $\mathrm{p}<0.01$, respectively). Fewer complete eyeblinks and more eyeblink attempts $(\mathrm{p}<0.01$ for both) were found in interpalpebral lens fits than in lid attachment fits. Fewer complete eyeblinks $(\mathrm{p}=0.02)$ and more incomplete eyeblinks $(\mathrm{p}=0.03)$ were found with lenses rated as large or optimal in size than with lenses rated as small.

Conclusions. GP lens wearers experiencing 3- and 9-o'clock staining showed a different eyeblink frequency for individual types of eyeblinks, but not for overall eyeblinks. Fewer complete eyeblinks, more incomplete eyeblinks and more eyeblink attempts were observed in GP wearers with 3- and 9-o'clock staining compared to wearers with minimal staining and non-wearers. In addition, some individual contact lens variables were associated with more incomplete eyeblinks.

Key words: Corneal desiccation, GP contact lenses, 3- and 9-o'clock corneal staining, eyeblink frequency, amplitude of eyeblink, lens fit, lens edge thickness, lens size 
With currently available designs and materials, the majority of contact lenses can be fitted and worn successfully. But one of the most frequently reported remaining problems relates to dryness symptoms, possibly affecting up to $50 \%$ of all contact lens wearers. ${ }^{1}$ This occurs in both hydrogel and rigid gas permeable (GP) lens wear but manifestation, etiology and severity are different. ${ }^{2-4}$ In GP lens wear the dryness symptoms may be associated with 3- and 9-o'clock staining. ${ }^{2,3}$

Based on theoretical considerations and clinical observation, alteration in eyeblink behavior during GP lens wear can be a possible risk factor for the development of 3- and 9-o'clock staining (the eyeblink theory). ${ }^{5-7}$ The assumption is made that discomfort inhibits eyeblinking, which will stimulate tear evaporation and this in turn can promote the development of 3- and 9-o'clock staining. ${ }^{1,3}$

The term 3- and 9-o'clock staining, ${ }^{3}$ (also called peripheral corneal staining, ${ }^{8}$ peripheral corneal desiccation, ${ }^{9} 4$ and 8 o'clock staining, ${ }^{10}$ juxtaposition staining ${ }^{11}$ or persistent nasal and temporal stippling ${ }^{12}$ ) describes the condition of corneal epithelial staining in the nasal and temporal region of eyes wearing GP contact lenses. This condition has been reported in the literature since the introduction of rigid corneal lenses in the early 1950s. ${ }^{11-13}$ Some degree of 3- and 9-o'clock staining has been described in as many as $80 \%$ of GP lens wearers, ${ }^{14}$ but it is estimated to be of clinical significance in about $10-15 \% .^{14,15}$

To the best of our knowledge no studies on the relationship between frequency and type of eyeblink and 3- and 9-o'clock staining have been reported so far. The main aim of this study was to test the hypothesis that frequency of different types of eyeblinks in GP lens wearers experiencing 3- and 9-o'clock staining is different from that in GP lens wearers without substantial 3- and 9-o'clock staining and non-lens wearers. In addition, specific contact lens variables that could potentially influence eyeblink frequency were analyzed.

\section{Methods}

\section{Subjects and Lens Wear}

This study involved 81 subjects. Of these, 55 were GP contact lens wearers, both with and without 3- and 9-o'clock staining, from the database of a large contact lens practice in the Netherlands. The control group consisted of 26 non-lens wearing patients of the same practice. The GP lens wearers included in this study were long term, successful contact lens wearers who were seen as part of a regular checkup schedule. 
Information about the ocular history was obtained by interviewing the subjects. All tested eyes were free of pathological eye conditions, including pathological dry eye conditions. No additional dry eye testing was performed. Subjects were asked to wear their lenses for a minimum of one hour on the day of the examination visit. All procedures in this study were conducted in accordance with the Declaration of Helsinki (2000), and all subjects signed an informed consent form prior to participation in the study.

\section{Corneal Staining}

A detailed slitlamp (Nikon FS-3) examination was performed using fluorescein (Fluorets, Chauvin), cobalt blue light and a Wratten \#12 yellow barrier filter.

A specially designed image grading scale for 3- and 9-o'clock staining, developed at the University of Maastricht, was used to grade all eyes in decimals on a $0-4$ point scale (Figure 1).

A combination of a background photograph and an overlaid pictorial representation was used to create the desired images. The major advantage of photographic images over pictorial scales is that they represent a more realistic clinical picture. However, when developing photographic scales it is a challenge to find a picture that represents exactly the desired grade. Furthermore, differences in anatomy between eyes, lighting conditions and other observational variables hamper the clinical assessment and interpretation of the photographic grades. All these disadvantages are overcome by pictorial scales in which the condition of interest is sketched at different levels of severity. This option obviously lacks a level of realism, which might be a disadvantage when used in a clinical setting. Therefore, a combination of a background photograph with an overlaid pictorial representation of staining was used to benefit from the advantages both systems have to offer.

The five-point image grading scale for 3-and 9-o'clock staining was developed based on currently available descriptive scales for 3- and 9-o'clock staining and available general image grading scales for corneal staining. To acknowledge any potential variance in staining intensity versus the extent of the staining, slight differences in staining (deeper and smaller versus larger and more superficial) are marked nasally and temporally in the developed scale. The input of 182 contact lens practitioners in the Netherlands was used to further refine the scale. The resulting scale was objectively tested for linearity by James Wolffsohn at Aston University, Birmingham, United Kingdom using previously validated image analysis techniques. ${ }^{16}$ To allow the use of interpolations between grading images (such as one tenth of a unit), which can increase discrimination, ${ }^{17}$ there should be a linear 
incremental increase in severity between grading scale units. Without a linear relationship, half or 0.1 step grading increments will not correspond to the exact grade the practitioner intends to assign. ${ }^{17}$
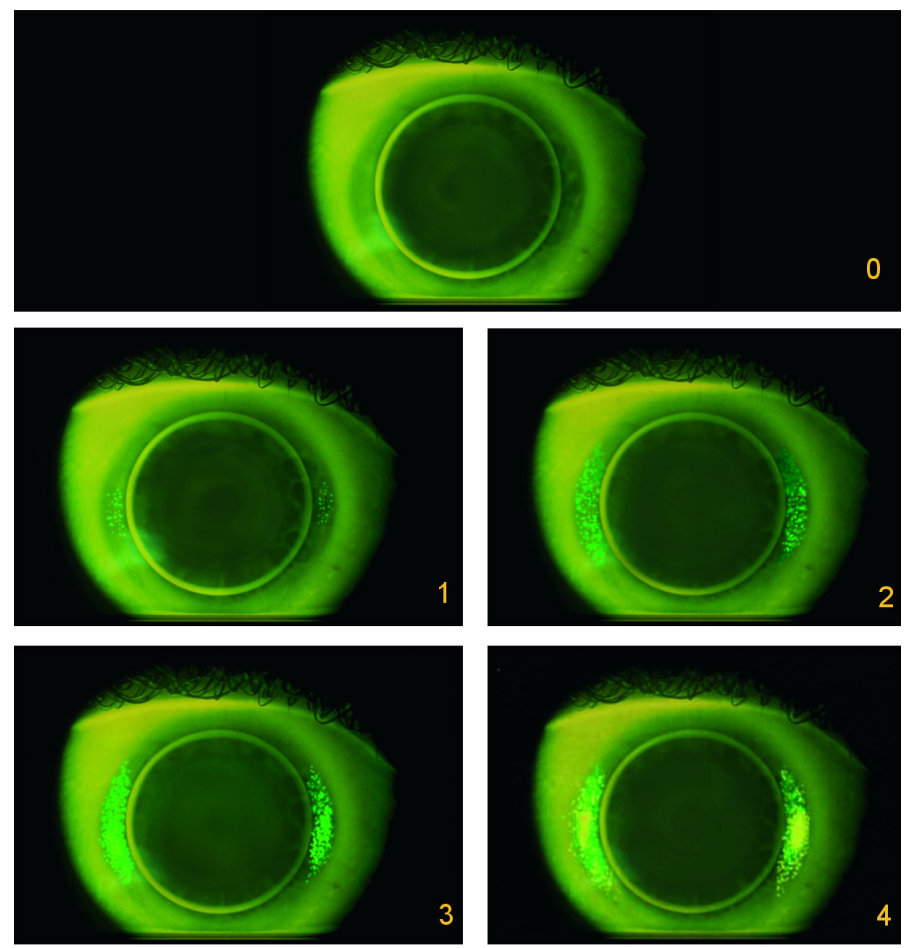

FIGURE 1. The specially designed image grading scale for 3- and 9o'clock staining developed at the University of Maastricht that was used to grade all eyes in decimals on a $0-4$ point scale in this study. A combination of a background photograph and an overlaid pictorial representation was used to create the desired images of this grading scale.

The scale showed a linear increase between grade units for edge detection of $r^{2}=0.99$. For comparison, ${ }^{17}$ the linearity of corneal staining for the Efron grading scale ${ }^{18}$ using edge detection was $r^{2}=0.49$ while it was $r^{2}=0.51$ for the CCLRU (Cornea and Contact Lens Research Unit, Sydney Australia) scale. ${ }^{19}$ For relative coloration of corneal staining the linearity of the 3- and 9-o'clock staining scale was $\mathrm{r}^{2}=0.70$, while the Efron grading scale achieved $\mathrm{r}^{2}=0.55$ and the CCLRU scale $r^{2}=0.86$. For green coloration the linearity was high as well $\left(r^{2}=0.96\right)$, but no corresponding data are available for the Efron and CCLRU scales for comparison. In summary, the developed 3- and 9-o'clock staining grading scale proved to be linear in nature and therefore it seems appropriate to interpolate between scale images 
and use small incremental steps to increase discrimination when grading this condition using this scale.

The maximum amount of staining in both eyes of each subject in this study was rated in a masked fashion by two investigators separately. Using the described scale, the interobserver correlation for grading corneal staining was $\mathrm{r}=0.93(\mathrm{p}<0.01)$. If different results were found between the two investigators, the average of the two ratings was used for further analysis.

Three groups were identified: non-lens wearers formed the control group (group I), GP lens wearers with less than grade 1 staining in the three or nine o'clock position of the cornea were assigned to group II and GP lens wearers with significant 3-and 9-o'clock corneal staining (grade 1 or more in at least one eye) were assigned to group III.

\section{Eyeblink Evaluation}

Subjects were seated in a comfortable, high-backed chair and asked to direct their gaze towards a 35-mm-high, vertically orientated black cross on a white background of the wall that was at a $2.5 \mathrm{~m}$ distance. The chair height was adjusted so that the viewing was comfortable in primary eye gaze. The experimental conditions were adopted from previous eyeblink recordings as described by Doughty et al. ${ }^{20}$ The ambient lightning around the subject was set at 600 lux and the illumination at the target was 350 lux. Room temperature (mean $21.4 \pm \mathrm{SD} 1.2^{\circ} \mathrm{C}$ ) and humidity (mean $53 \pm$ SD 3\%) were monitored. All measurements were taken between 9am and $6 \mathrm{pm}$. Barbato et al. ${ }^{21}$ found that spontaneous eye-blink rate showed a stable pattern in morning, midday and afternoon hours when analyzing diurnal variation in eyeblink rate.

A Hitachi HVC20A camera was located at head height, but at an angle and away from the subject so not to directly interfere with the visual task presented. The camera was linked to a high quality Panasonic NV-FS100 HQ video recorder. The subjects were not specifically notified that their eyeblinks were being monitored, but rather that the study was a general observation of the eye and face dynamics while looking straight ahead. The recording lasted for five minutes, and the subjects were not given clues as to the passage of time. During the recording, no conversation in any form took place and the investigator was situated outside the field of view of the subject.

The video recordings were transferred to DVD format for analysis in a masked fashion digitally using Intervideo Win DVD@ software, version 4.0 DXVA. Every 
eyeblink action of all 81 patients was monitored: the exact time in 1/100 of a second accuracy of each eyeblink was registered at the time of maximum amplitude of the eyeblink action. The investigator was masked as to the subject's amount of staining during eyeblink analysis. The maximum amplitude of each eyeblink itself was measured as well, manually from the computer screen and this was compared to the measured total palpebral aperture height. Based on this, every eyeblink within the five minute period was assigned to one of three eyeblink categories: complete eyeblinks (the upper eyelid covering between 91 and 100\% of the palpebral aperture), incomplete eyeblinks (covering between 40 and $90 \%$ of the palpebral aperture) and eyeblink attempts (any eyelid action covering between zero and $39 \%$ of the palpebral aperture).

\section{Lens Fit}

To analyze specific contact lens variables that could be related to eyeblink frequency, a video recording of each contact lens and its fluorescein pattern was generated with a CCD camera (Mitsubishi CCD-100E) and a DVD recorder (Panasonic DMR E30). The same slitlamp technique (including the use of fluorescein) was used as described for observing corneal staining.

To assess the influence of the upper eyelid in relation to the lens edge, all lens fits were rated as either interpalpebral (the contact lens is positioned between the eyelids during inter-eyeblink periods) or lid attachment (the lens stays under the upper eyelid in between eyeblinks). If an interpalpebral lens fit was seen in only one eye (which was the case in three of the 55 subjects) then this subject was considered as having an interpalpebral lens fit. The assumption was made that in these cases there is substantial interaction of the upper eyelid with the edge of the lens during an eyeblink, even though this happens in only one eye.

To analyze the lens-to-cornea fit in relation to eyeblink frequency, the post-lens tear layer thickness (using fluorescein) in the vertical direction at the edge of the lens was graded on a five point scale as either optimal (0), slightly too thick/thin (+/-) but acceptable (grade 1 ) or excessively too thick/thin (grade 2 ). The lens was held in a static, central position on the cornea. A grade 2 thick tear layer at the edge of the lens was considered a flat lens fit in this study. To compare the eyeblink frequency in flat lens fits compared to other lens fits, subjects with a flat lens fit in one eye and subjects with a flat lens fit in both eyes were identified and evaluated separately.

The effect of edge shape is very complex to analyze, especially in a clinical setting. To a lesser extent the same is true for edge thickness. Edge shape and edge thick- 
ness were not further analyzed due to the large variation of possibilities and the limitations with regard to evaluating this in clinical practice. Instead, the spherical equivalent of the refraction (average of the two eyes) was analyzed in relation to 3and 9-o'clock staining.

Lens size was subjectively rated on-eye as optimal, large or small, and is referred to as corneal coverage. An 11:9 corneal coverage ratio between the vertical visible iris diameter and total lens diameter was considered optimal. If the corneal coverage rating in two eyes was not the same (which was the case in five of the 55 subjects) then the larger of the two ratings was used for further analysis. Corneal coverage was the preferred method (as opposed to analysis of absolute lens diameter) because it reflects best whether the size of the lens is appropriate for a particular cornea.

\section{Data Analysis}

Statistical analysis of the data was performed using Statistical Package for Social Sciences (SPSS) version 11.5. Since the distribution of data was mostly nonGaussian in nature, non-parametric Mann-Whitney (MW) and Kruskal-Wallis $(\mathrm{KW})$ tests were used for statistical analysis. Power analysis was used to ensure an adequate sample size. ${ }^{22} \mathrm{~A}$ critical $\mathrm{p}$ value of 0.05 was used to denote statistical significance. Because of use of multiple comparisons when analyzing the three different staining groups individually, a Bonferroni correction was applied such that $\mathrm{p}=0.017(0.05 / 3)$ was taken as the critical level for statistical significance for comparison between staining groups II and III. Correlations between variables were analyzed by calculating Spearman's rho. The number of eyeblinks over the five minute period was converted to eyeblinks per minute and presented as the median of eyeblinks.

\section{Results}

\section{Subjects and Lens Wear}

The mean age of subjects was $40.7 \pm$ SD 13.4 years of age (range 14.8 to 72.4 years). Group I consisted of 26 subjects $(33.8 \pm 10.9$ years of age, range 18.5 to 56.6), group II consisted of 25 subjects ( $41.9 \pm 16.1$, range 14.9 to 72.4 ) and 30 subjects $(42.9 \pm 11.2$, range 21.6 to 68.0$)$ formed group III. No correlation between age and overall eyeblink frequency was found $(\mathrm{r}=-0.05, \mathrm{p}=0.63)$.

The proportion of male to female subjects was $31 \%$ male versus $69 \%$ female, which is a realistic reflection of the gender ratio of lens fits around the world. ${ }^{23}$ No difference in the overall eyeblink frequency was found in relation to gender (MW $\mathrm{p}=0.50$ ). The median wearing time of the lenses on the day of examination was 3.5 
(1.0 to 11.0) hours. A low correlation was found between hours of lens wear at the time of examination and corneal staining $(r=0.31, p=0.02)$.

\section{Eyeblink Frequency}

Table 1 represents an overview of eyeblink frequencies overall and for different types of eyeblinks. The median overall eyeblink frequency per minute (all eyeblink actions together) in group I (non-lens wearers) was 16.8, in group II this was 15.8 and 17.2 in group III (Figure 2). No difference between the three groups for overall eyeblink frequency was seen (KW $\mathrm{p}=0.99)$.

Major differences were seen in eyeblink frequencies for different types of eyeblinks. The median frequency for complete eyeblinks (Figure 3) in group I was 14.4, while this was 12.2 in group II and 4.6 in group III. This difference was statistically significant $(\mathrm{KW} \mathrm{p}<0.01)$. The reverse pattern was seen for incomplete eyeblinks (Figure 4): the median incomplete eyeblink frequency was 1.4 in groups I and II, while it was 4.0 in group III. This difference was statistically significant (KW p=0.03). Eyeblink attempt distribution showed a similar pattern to incomplete eyeblinks (Figure 5). The median eyeblink attempt frequency was 0.2 in groups I and II, while this was 1.6 in group III. For eyeblink attempts a statistically significant difference was also found (KW $\mathrm{p}<0.01)$.

TABLE 1. Median eyeblink frequency per minute for overall, complete, incomplete eyeblinks and eyeblink attempts in non-lens wearers (group I), GP lens wearers with less than grade 1 corneal staining (group II) and GP lens wearers with grade 1 or more corneal staining (group III)

\begin{tabular}{lllcll}
\hline Group & Eyeblink type & $\mathrm{N}$ & Median & $\begin{array}{l}\text { Interquartile } \\
\text { range }\end{array}$ & $\begin{array}{l}\text { Minimum- } \\
\text { Maximum }\end{array}$ \\
\hline group I & overall & 26 & 16.8 & 13.0 & 2.6 to 47.4 \\
& complete1 $^{2}$ & 26 & 14.4 & 12.8 & 2.4 to 47.4 \\
& incomplete $^{2}$ & 26 & 1.4 & 3.0 & 0 to 14.8 \\
& attempt $^{1}$ & 26 & 0.2 & 0.2 & 0 to 0.8 \\
group II & & & & & \\
& overall $^{*}$ & 25 & 15.8 & 10.3 & 2.4 to 30.8 \\
& complete $^{1}$ & 25 & 12.2 & 14.4 & 1.6 to 22.2 \\
& incomplete $^{2}$ & 25 & 1.4 & 4.1 & 0 to 11.4 \\
& attempt $^{1}$ & 25 & 0.2 & 0.6 & 0 to 11.2 \\
group III & & & & & 3.4 to 50.4 \\
& overall $^{*}$ & 30 & 17.2 & 13.8 & 0 to 33.4 \\
& complete $^{1}$ & 30 & 4.6 & 13.0 & 0 to 34.4 \\
& incomplete $^{2}$ & 30 & 4.0 & 7.8 & 0 to 19.8 \\
\hline
\end{tabular}

Superscript numbers indicate statistically significant differences between groups for different eyeblink types, using Kruskal Wallis test: $1 \mathrm{p}<0.01,2 \mathrm{p}<0.05$ 

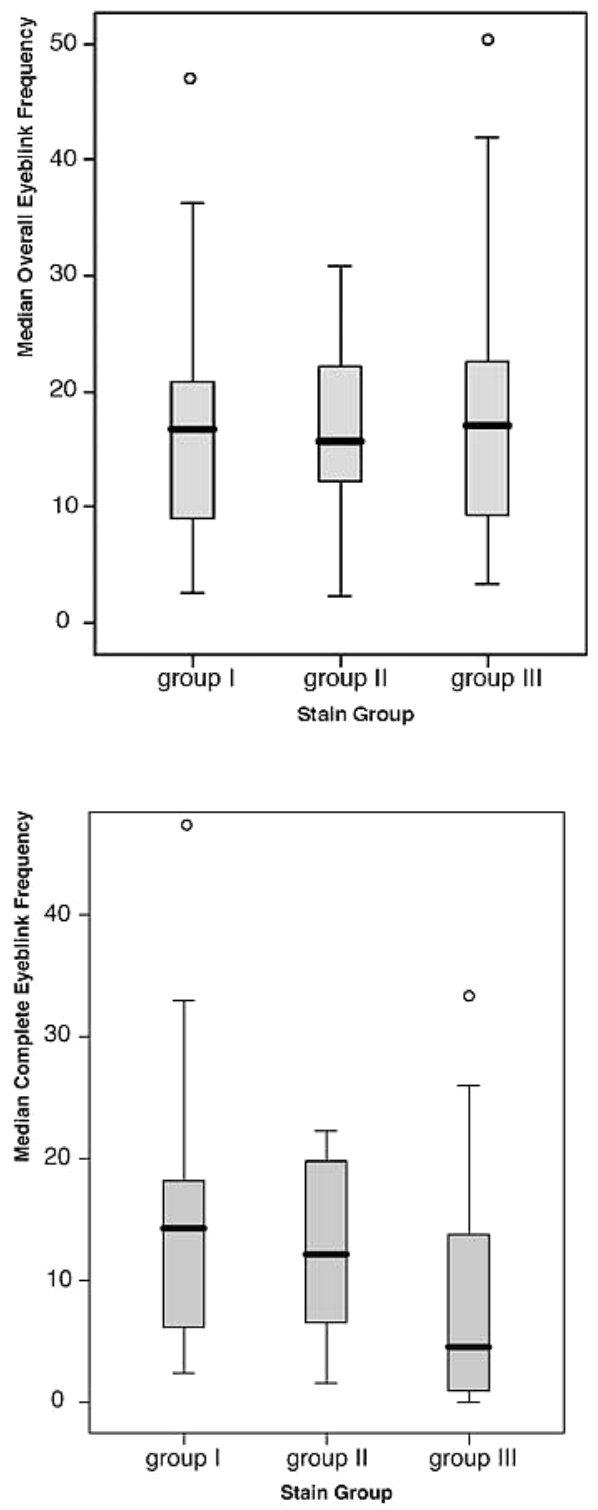

FIGURE 2. Median overall eyeblink frequency per minute in non-lens wearers (group I), GP lens wearers with less than grade 1 corneal staining (group II) and GP lens wearers with grade 1 or more corneal staining (group III). Box indicates interquartile range; a line through this box shows the median. The whiskers extend out to the lowest and highest case that is "not considered to be an outlier or an extreme value"; (o) indicates outlier (1.5-3.0 box lengths away from box edge); $\left(^{*}\right)$ indicates extreme (over 3.0 box lengths away from box edge).

FIGURE 3. Median complete eyeblink frequency per minute in non-lens wearers (group I), GP lens wearers with less than grade 1 corneal staining (group II) and GP lens wearers with grade 1 or more corneal staining (group III). Box indicates interquartile range; a line through this box shows the median. The whiskers extend out to the lowest and highest case that is "not considered to be an outlier or an extreme value"; (o) indicates outlier (1.5-3.0 box lengths away from box edge); $\left(^{*}\right)$ indicates extreme (over 3.0 box lengths away from box edge). 

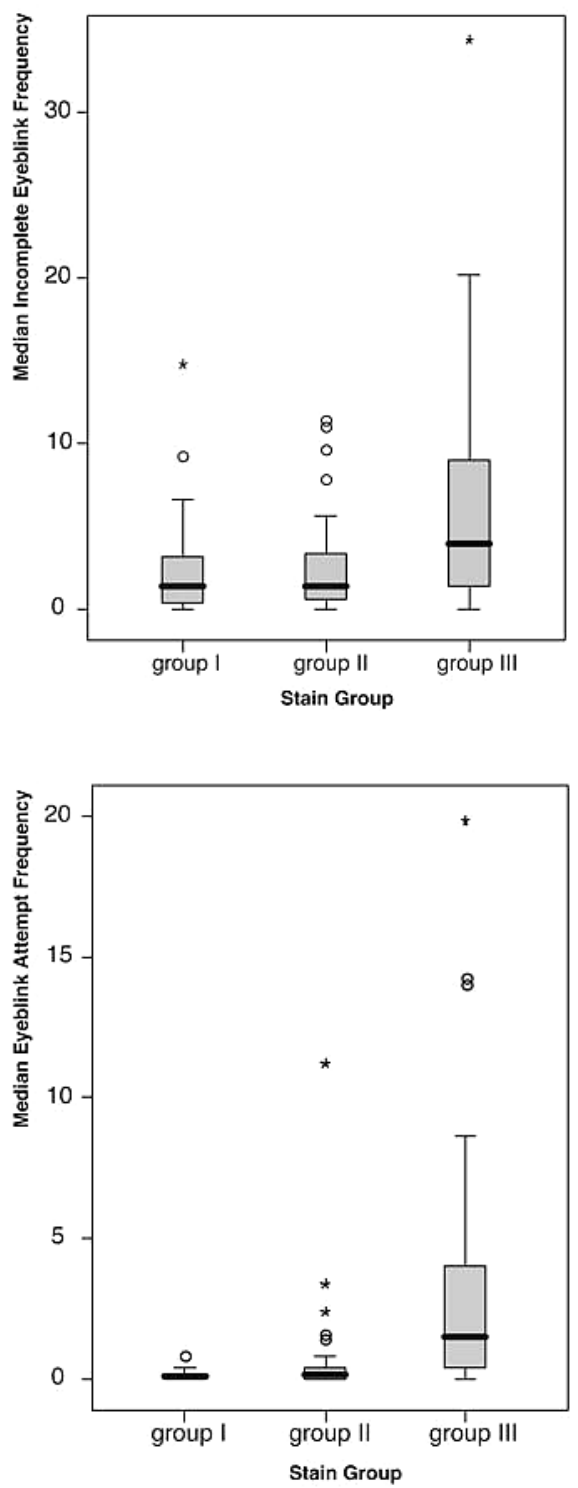

FIGURE 4. Median incomplete eyeblink frequency per minute in non-lens wearers (group I), GP lens wearers with less than grade 1 corneal staining (group II) and GP lens wearers with grade 1 or more corneal staining (group III). Box indicates interquartile range; a line through this box shows the median. The whiskers extend out to the lowest and highest case that is "not considered to be an outlier or an extreme value"; (o) indicates outlier (1.53.0 box lengths away from box edge); $\left({ }^{*}\right)$ indicates extreme (over 3.0 box lengths away from box edge).

FIGURE 5. Median eyeblink attempt frequency per minute in non-lens wearers (group I), GP lens wearers with less than grade 1 corneal staining (group II) and GP lens wearers with grade 1 or more corneal staining (group III). Box indicates interquartile range; a line through this box shows the median. The whiskers extend out to the lowest and highest case that is "not considered to be an outlier or an extreme value"; (o) indicates outlier (1.53.0 box lengths away from box edge); $\left(^{*}\right)$ indicates extreme (over 3.0 box lengths away from box edge).

When comparing GP lens wearers (groups II and III combined, $\mathrm{n}=55$ ) to non-lens wearers (group I), no difference in overall eyeblink frequency was found: 16.8 (range 42.4 to 50.4 ) versus 16.8 (2.6 to 47.4 ) respectively (MW p=0.97). GP lens wearers showed fewer complete eyeblinks in this study than non-lens wearers: 7.6 (0 to 33.4) versus 14.4 (2.4 to 47.4 ) respectively (MW $\mathrm{p}=0.02$ ). GP lens wearers also showed more incomplete eyeblinks than non-lens wearers, 2.6 (0 to 34) versus 1.4 ( 0 to 15$)$ respectively, but this was not statistically significantly ( $\mathrm{MW} \mathrm{p}=0.09$ ). 
Slightly more eyeblink attempts were found in GP lens wearers than in non-lens wearers: 0.6 ( 0 to 19.8 ) versus 0.1 ( 0 to 0.8 ) respectively ( $\mathrm{MW} \mathrm{p}<0.01$ ).

When GP lens wearers with grade 1 or more corneal staining (group III) were compared to GP lens wearers with less than grade 1 corneal staining (group II), no difference in overall eyeblink frequency was found: 17.2 versus 15.8 respectively (MW $\mathrm{p}=0.99$ ). However, fewer complete eyeblinks were seen in group III than in group II: 4.6 versus 12.2 respectively $(\mathrm{MW} \mathrm{p}<0.01)$. More incomplete eyeblinks were seen in group III than in group II: 4.0 versus 1.4 respectively, but this was not statistically significant (MW $\mathrm{p}=0.06$ ). More eyeblink attempts were seen in group III than in group II: 1.6 versus 0.2 respectively (MW $\mathrm{p}<0.01$ ).

\section{Lens Fit}

Of all lens-wearing subjects, 16 were identified as having an interpalpebral lens fit and 39 as having a lid attachment fit (Table 2). A lower overall eyeblink frequency was found for interpalpebral lens fits than for lid attachment fits: 12.2 versus 17.8 respectively, but this difference was not statistically significant (MW $\mathrm{p}=0.11$ ). Considerably fewer complete eyeblinks were found for interpalpebral lens fits than for lid attachment fits: 4.4 versus 12.6 respectively ( $M W \mathrm{p}<0.01$ ). More incomplete eyeblinks were seen for interpalpebral fits than for lid attachment fits, but this was not statistically significant: 4.2 versus 1.8 respectively (MW p=0.29). Also, more eyeblink attempts were seen for interpalpebral fits than for lid attachment fits: 2.6 versus 0.2 respectively $(\mathrm{MW} \mathrm{p}<0.01)$.

To further evaluate the effect of the upper eyelid interaction with the lens edge, subjects with a flat lens fit in both eyes $(\mathrm{n}=12)$ were identified as well as subjects with a flat lens fit in one eye only $(n=15)$. These subjects were compared to subjects not showing a flat lens fit in either eye $(n=28)$. No difference in overall eyeblink frequency between the three defined lens fit groups (Table 3) was found (KW $\mathrm{p}=0.16)$. The highest complete eyeblink frequency (15.2) was seen in subjects with one flat lens fit while lower frequencies were found for subjects with a flat lens fit in both eyes and subjects with no flat lens fits: 5.6 and 6.0 respectively (KW $\mathrm{p}<0.01)$. The incomplete eyeblink frequency was almost equal for all three groups $(\mathrm{KW} \mathrm{p}=0.49)$. More eyeblink attempts were seen in subjects with a flat lens fit in both eyes than in the other groups, but this was not statistically significant (KW $\mathrm{p}=0.22$ ). 
TABLE 2. Median eyeblink frequency per minute for overall, complete, incomplete eyeblinks and eyeblink attempts in lid attachment lens fits and interpalpebral lens fits

\begin{tabular}{lllcll}
\hline Lens fit type & Eyeblink type & $\mathrm{N}$ & Median & $\begin{array}{l}\text { Interquartile } \\
\text { range }\end{array}$ & $\begin{array}{l}\text { Minimum- } \\
\text { Maximum }\end{array}$ \\
\hline lid attachment & overall & 39 & 17.8 & 10.8 & 2.4 to 50.4 \\
& complete $^{1}$ & 39 & 12.6 & 17.6 & 0 to 33.4 \\
& incomplete $^{\text {interpalpebral }}$ & 39 & 1.8 & 6.6 & 0 to 34.4 \\
& attempt $^{1}$ & 39 & 0.2 & 1.4 & 0 to 19.8 \\
& overall $^{1}$ & 16 & 12.2 & 10.1 & 4.8 to 27.4 \\
& complete $^{1}$ & 16 & 4.4 & 4.1 & 0 to 12.2 \\
& incomplete $^{1}$ & 16 & 4.2 & 8.8 & 0 to 20.2 \\
& attempt $^{1}$ & 16 & 2.6 & 4.2 & 0 to 8.6 \\
\hline
\end{tabular}

Superscript numbers indicate statistically significant differences between groups for different eyeblink types, using Mann Whitney test: $1 \mathrm{p}<0.01$

TABLE 3. Median eyeblink frequency per minute for overall, complete, incomplete eyeblinks and eyeblink attempts in subjects with no flat lens fit, a flat lens fit in one eye and a flat lens fit in both eyes

\begin{tabular}{lllrrl}
\hline Lens fit & Eyeblink type & $\mathrm{N}$ & Median & $\begin{array}{l}\text { Interquartile } \\
\text { range }\end{array}$ & $\begin{array}{l}\text { Minimum- } \\
\text { Maximum }\end{array}$ \\
\hline no flat lens fit & overall & 28 & 15.6 & 10.7 & 2.4 to 42.0 \\
& complete $^{1}$ & 28 & 6.0 & 10.7 & 0 to 33.4 \\
& incomplete $^{28}$ & 2.8 & 7.5 & 0 to 20.2 \\
& attempt & 28 & 0.4 & 3.5 & 0 to 19.8 \\
one eye flat lens fit & overall & 15 & 19.4 & 8.2 & 9.8 to 50.4 \\
& complete & 15 & 15.2 & 11.4 & 2.6 to 26.0 \\
& incomplete $^{1}$ & 15 & 2.0 & 3.6 & 0 to 34.4 \\
& attempt & 15 & 0.2 & 1.6 & 0 to 3.6 \\
\hline two eyes flat lens fit & overall & 12 & 12.6 & 13.3 & 3.4 to 27.4 \\
& complete & 12 & 5.6 & 11.3 & 0 to 22.4 \\
& incomplete & 12 & 1.8 & 7.6 & 0 to 18.4 \\
& attempt & 12 & 1.6 & 2.9 & 0 to 6.0 \\
\hline
\end{tabular}

Superscript numbers indicate statistically significant differences between groups for different eyeblink types, using Kruskal Wallis test: $1 \mathrm{p}<0.01$

With regard to corneal coverage, 37 lenses were rated as optimal, eight as small and ten as large (Table 4). When looking at overall eyeblink frequency, no statistically significant difference was found (KW p=0.37). Fewer complete eyeblinks were seen in large and optimal rated lenses than in small rated lenses: 6.2 and 5.6 versus 21.0 respectively $(\mathrm{KW} \mathrm{p}=0.02)$. Most incomplete eyeblinks were found in large rated lenses, followed by optimal rated lenses and small rated lenses: 7.4, 2.4 and 0.6 respectively $(\mathrm{KW} \mathrm{p}=0.03)$. Most eyeblink attempts were seen in large rated lenses, 
followed by optimal and small rated lenses, but no statistically significant difference was found (KW p=0.29).

The correlation between the spherical equivalent of the refraction and different types of eyeblinks was analyzed. The spherical equivalent of the refraction ranged from $-0.50 \mathrm{D}$ to $-10.00 \mathrm{D}$ for myopic subjects (mean $-3.73 \pm \mathrm{SD} 1.66 \mathrm{D}$ ) and from $+2.25 \mathrm{D}$ to $+7.00 \mathrm{D}(+4.23 \pm 1.35 \mathrm{D})$ for hyperopic subjects. Low correlations or no correlation at all between ametropia and overall eyeblinks, complete eyeblinks, incomplete eyeblinks and eyeblink attempts were found (Spearman's rho ranging between $\mathrm{r}=-0.31, \mathrm{p}=0.02$ and $\mathrm{r}=0.19, \mathrm{p}=0.16$ ).

TABLE 4. Median eyeblink frequency per minute for overall, complete, incomplete eyeblinks and eyeblink attempts for small, optimal and large ratings of corneal coverage

\begin{tabular}{llrrrl}
\hline $\begin{array}{l}\text { Corneal cover- } \\
\text { age }\end{array}$ & Eyeblink Type & $\mathrm{N}$ & Median & $\begin{array}{l}\text { Interquartile } \\
\text { range }\end{array}$ & $\begin{array}{l}\text { Minimum- } \\
\text { Maximum }\end{array}$ \\
\hline small & overall & 8 & 21.2 & 8.6 & 8.6 to 23.4 \\
& complete $^{2}$ & 8 & 21.0 & 14.2 & 6 to 22.4 \\
& incomplete $^{2}$ & 8 & 0.6 & 2.8 & 0 to 4.2 \\
optimal & attempt & 8 & 0.2 & 0.4 & 0 to 8.4 \\
& overall & 37 & 14.2 & 11.7 & 2.4 to 50.4 \\
& complete & 37 & 5.6 & 13.0 & 0 to 26.0 \\
& incomplete & 37 & 2.4 & 8.6 & 0 to 34.4 \\
large & attempt & 37 & 0.8 & 3.4 & 0 to 19.8 \\
& overall & 10 & 18.4 & 19.0 & 4.2 to 42.0 \\
& complete & 10 & 6.2 & 12.2 & 1.0 to 33.4 \\
& incomplete & 10 & 7.4 & 5.6 & 0.2 to 20.2 \\
\hline
\end{tabular}

Superscript numbers indicate statistically significant differences between groups for different eyeblink types, using Kruskal Wallis test: 2 p $<0.05$

\section{Discussion}

The number of overall eyeblinks was essentially the same for non-lens wearers and GP lens wearers in our study. Also, there was no difference in overall eyeblink frequency between lens wearers with less than grade 1 corneal staining (group II) and lens wearers with grade 1 or more corneal staining (group III). Eyeblink inhibition has been mentioned as one of the possible causes of 3- and 9-o'clock staining. ${ }^{1,5,7,24,25}$ If so, it seems questionable whether this is reflected in the overall eyeblink frequency. Based on our findings, evaluation of overall eyeblink frequency does not seem to be a useful measurement in GP lens wear when managing 3- and 9-o'clock staining. 
When the different types of eyeblinks were analysed individually, i.e. the eyeblink quality was taken into account, substantial differences were noted. GP contact lens wearers showed fewer complete eyeblinks and more eyeblink attempts than nonlens wearers. This effect can almost exclusively be attributed to group III subjects (GP lens wearers with substantial 3- and 9-o'clock staining). Subjects in group III showed a lower complete eyeblink frequency and a higher eyeblink attempt frequency compared to subjects in group II. This finding suggests a relationship between the completeness of eyeblinks and 3- and 9-o'clock staining. If so, it may support the eyeblink theory concerning the etiology of 3- and 9-o'clock staining, but with the qualification that it is not the overall frequency of eyeblinks that is causing the effect but the quality of the eyeblinks that plays a crucial role.

Discomfort upon eyeblinking because of interaction of the eyelid with the lens edge can possibly result in inhibition of the eyeblink, which in turn can lead to increased tear evaporation, corneal desiccation and thus corneal staining. ${ }^{26}$ If this is the case, alteration of some of the lens parameters may influence this effect. Type of lens fit (interpalpebral versus lid attachment), lens-to-cornea fit (flat lens fits), corneal coverage (lens diameter) and possibly the spherical equivalent of the refraction all may, in theory, interfere with eyeblink behavior.

Considerably fewer complete eyeblinks and more eyeblink attempts were found for interpalpebral lens fits than lid attachment fits, while fewer complete eyeblinks and more incomplete eyeblinks were found in optimal and large rated lenses than in small rated lenses. For flat lens fits no association with frequency or completeness of eyeblinks was found. Furthermore, no relationship for any of these variables with overall eyeblink frequency was found.

Based on these findings it appears that some individual GP lens variables may have an influence on the completeness of eyeblinks. If so, it seems advisable to minimize the interaction of the upper eyelid with the lens edge when fitting GP lenses. This could mean to strive for lid attachment fits and smaller lens sizes. Flat lens fits did not show an effect on eyeblink behaviour, although this effect has been suggested in the literature. ${ }^{3}$ It should be kept in mind that it is difficult to analyze the effect of contact lens variables such as lens-to-cornea fit if this is present in one eye only, while eyeblink frequency is a variable that is measured for both eyes together.

The low number of participants in the different subcategories is another limitation when analyzing the contact lens variables in this study. It would, for instance, be of particular interest to investigate the effect of flat fitting lenses in interpalpebral lens fits, but the sample size in the individual subcategories was too low for such analy- 
sis. Future prospective studies with larger sample sizes representing specific groups of flat and steep fitting lenses - in both eyes - could possibly differentiate better between the lens related variables in this regard.

No association between the spherical equivalent of the refraction and frequency or completeness of eyeblinks was found. Higher myopic correction generally is thought to be associated with a thicker lens edge, and with a higher hyperopic correction typically a thinner lens edge is expected. ${ }^{27}$ This effect may be limited due to the fact that high minus lenses often utilize a lenticular edge design to reduce the lens edge thickness. However, generally the periphery of high minus lenses still is considered to be thicker than for low minus or hyperopic lenses. In summary, there may be a possible relationship between edge thickness and spherical equivalent of the refraction, but the exact nature of this relationship is unclear. Based on the findings of this study no firm statement can be made regarding edge thickness in relation to 3- and 9-o'clock staining.

Differences in the eyeblink frequency and completeness of eyeblinks in contact lens wear have been cited in the literature, ${ }^{28-31}$ but the number of studies on this subject is limited with regard to GP lens wear. Only a few reports are available, and they all concern hard (PMMA) lens wear and only focus on the short term effects of these lenses. Only one study used a categorization of eyeblinks to analyze eyeblink behavior in a small $(\mathrm{n}=7)$ study.

York et al. ${ }^{32}$ in 1971 found an increase in the eyeblink rate, measured nine days after hard lenses were fitted to neophyte lens wearers, but no differentiation in eyeblink type was made. On the other hand, Brown et al..$^{30}$ in 1973 found a general decrease in eyeblink rate with hard lenses ten minutes after lens fit. They suggested that this could be the result of the patient's attempt to avoid contact between the upper eyelid and the lens edge. Hill and Carney ${ }^{33}$ studied seven subjects before and after fitting with hard contact lenses, and found an increase in eyeblink rate from 15.5 eyeblinks/minute to 23.2 eyeblinks/minute approximately three weeks after the lenses were fitted. No difference in distribution of eyeblink type before and after lens fit was found. Our results are not consistent with these earlier reports. The early studies all focused on the initial response to GP lens wear, and did not evaluate the relationship with 3- and 9-o'clock staining. Our study analyzed long term, successful contact lens wearers, involved subjects with and without 3- and 9o'clock staining, and specifically used eyeblink quality as a variable.

One of the difficulties when analyzing eyeblink frequency is the large variation of eyeblinks in individuals, as has been found in other studies. ${ }^{29,34,35}$ A similarly large 
range was found in our study, with a range of overall eyeblink frequency from 12 eyeblinks to as many as 252 eyeblinks over the five-minute test period. The range of the eyeblink frequency in non-GP lens wearers was not found to be different from GP lens wearers (2.6 to 47.4 eyeblinks per minute versus 2.4 to 50.4 eyeblinks per minute). The large variation in eyeblink rates makes it particularly difficult for practitioners to use eyeblink frequency as a tool in clinical practice.

The definition of an eyeblink is a discussion in itself. Usually an eyeblink is described in the literature as a 'discernible movement of the upper eyelid causing an obvious reduction in the size of the palpebral fissure, ${ }^{32,36}$ although it often remains unclear how large that discernible movement exactly is. ${ }^{28,30,37-39}$ Categorization of eyeblinks based on the amount of reduction in aperture height has been suggested by Abelson and Holly. ${ }^{36}$ They subdivided unforced eyeblinks into either complete eyeblinks, incomplete eyeblinks or twitch eyeblinks. The definition of complete eyeblinks was described as the upper eyelid covering more than two-thirds of the cornea. ${ }^{36}$ Incomplete eyeblinks were described as 'the descending upper eyelid covering less than two thirds of the cornea' and a twitch eyeblink was defined broadly as 'an almost undetectable small movement of the upper eyelid'. Some studies have used a 'forceful eyeblink' in addition to these as a separate category. This is described as a voluntary, forced eyeblink in which the lower eyelid is raised, producing a near squint..$^{35,36,39-41}$ This type of eyeblink appears to be very rare. ${ }^{33,35,36,40}$

Since the primary goal of this study was to investigate the frequency and completeness of eyeblinks in GP lens wearers, a specific classification for eyeblinks was used. The smallest category of eyeblinks in this study was defined as an eyeblink action not covering more than $40 \%$ of the total palpebral aperture. In this way, all eyeblinks possibly affected by the interaction between the upper eyelid and the superior part of the lens edge are incorporated even if this involves a small and/or low riding lens. The term used for this type of eyeblink was eyeblink attempt as opposed to twitch eyeblink, since it can be argued that this partial eyelid movement in GP lens wearers might in fact not be a real eyeblink but rather an attempt to do so. An eyeblink was classified as incomplete if the upper eyelid covered between $40 \%$ and $90 \%$ of the palpebral aperture. A complete eyeblink was defined as a complete or nearly complete eyeblink movement of the upper eyelid, covering between 90 and $100 \%$ of the palpebral aperture. One of the main goals of this study was to analyze any difference between complete and incomplete eyeblinks. The decision to choose $90 \%$ as an arbitrary borderline to distinguish between these two eyeblink types was a practical one: $10 \%$ was the minimum visible opening of the palpebral aperture that was reliably apparent to the observer; if the closure was more than $90 \%$ the eye appeared closed on the video recording. 
It is interesting to note that while the number of complete eyeblinks was statistically significantly lowest in GP lens wearers with 3- and 9-o'clock staining, this was caused in particular by an increase in eyeblink attempts and only to a lesser extent related to the incomplete eyeblink frequency. This may have to do with the interaction of the superior eyelid with the edge of the lens, causing more eyeblink attempts, but more studies in this regard are needed to confirm this.

Another point worth mentioning when studying eyeblink behavior is the possible awareness of the videotaping of the eyeblinks. This is a potential difficulty that has been considered previously. ${ }^{20,42}$ In order to overcome this as much as possible in our study, the experimental conditions were adopted from previous eyeblink recordings as described by Doughty. ${ }^{20}$ Still this cannot be excluded as a potential influential factor on eyeblink behavior. Further studies on the effect of the subject's awareness of the taping relative to eyeblink behavior seem required in this respect.

\section{Conclusions}

With currently available designs and materials the majority of contact lenses can be fitted and worn successfully. One of the most frequently reported remaining problems relates to dryness symptoms, which may manifest as 3- and 9-o'clock staining in GP lens wear. In this study, fewer complete eyeblinks, more incomplete eyeblinks and more eyeblink attempts were observed in GP wearers with 3- and 9o'clock staining compared to wearers with less staining and non-wearers. In addition, some individual GP contact lens variables in this study were associated with fewer complete eyeblinks and more incomplete eyeblinks or eyeblink attempts. Further research should aim to identify how to adjust these variables to minimize the potential interference with the frequency and completeness of eyeblinks.

\section{Acknowledgements}

The authors wish to thank Jantine Bos and Rob Faas of Groenhof Optiek in Amstelveen, the Netherlands, and Peter van Etten for their help during the study. Thanks are also due to Ineke Krijger for her comments on this paper.

The authors particularly thank James Wolffsohn for his help in analyzing the image grading scale.

Color printing of the grading scale was made possible by joint support from the European Federation of Contact Lens Industry (EFCLIN) and the Contact Lens Manufacturers Association in the United States (CLMA). 


\section{References}

1. Fonn D. Preventing contact lens dropouts. Contact Lens Spectrum 2002;17:43-51.

2. Efron N. Tear film dysfunction. In: Efron N, ed. Contact Lens Complications. Oxford: Butterworth-Heinemann, 1999:61-71.

3. Van der Worp E, De Brabander J, Swarbrick H, Nuijts R, Hendrikse F. Corneal desiccation in rigid contact lens wear: 3- and 9-o'clock staining. Optom Vis Sci 2003;80:280-90.

4. Little S, Bruce A. Role of post-lens tear film in the mechanism of inferior arcuate staining with ultrathin hydrogel lenses. CLAO J 1995;21:175-81.

5. Lowther GE. Dryness, Tears, and Contact Lens Wear: Clinical Practice in Contact Lenses. Boston: Butterworth-Heinemann, 1997:84-90.

6. Buch J. Peripheral corneal staining: a survey of RGP laboratories. Contact Lens Spectrum 1997;12(7):31-7.

7. Efron N. Blinking. In: Efron N, ed. Contact Lens Complications. Oxford: ButterworthHeinemann, 1999:3-9.

8. Andrasko G. Peripheral corneal staining: incidence and time course. Contact Lens Spectrum 1990;5(7):59.

9. Schnider C, Terry R, Holden B. Effect of patient and lens performance characteristics on peripheral corneal desiccation. J Am Optom Assoc 1996;67:144-50.

10. Arner RS. Corneal contact lens design by minimal corneal insult. J Am Optom Assoc 1969;40:308-9.

11. Barabas R, Fontana A. Juxtaposition staining. Contacto 1967;11:3-6.

12. Graham R. Persistent nasal and temporal stippling. Contacto 1968;12:20-12.

13. Steele E. Observations on the fitting of corneal contact lenses. Am J Optom Arch Am Acad Optom 1959;36:194-9.

14. Solomon J. Causes and treatments of peripheral corneal desiccation. Contact Lens Forum 1986;11:30-6.

15. Ghormley N, Bennett E, Schnider C. Corneal desiccation-clinical management. Int Contact Lens Clin 1990;17:5.

16. Wolffsohn J, Purslow C. Clinical monitoring of ocular physiology using digital image analysis. Contact Lens Ant Eye 2003;26:27-35.

17. Wolffsohn J. Incremental nature of anterior eye grading scales determined by objective image analysis. Br J Ophthalmol 2004;88:1434-8.

18. Efron N. Grading scales for contact lens complications. Ophthalmic Physiol Opt 1998;18:1826.

19. Terry RL, Schnider CM, Holden BA et al. CCLRU standards for success of daily and extended wear contact lenses. Optom Vis Sci 1993;70:234-43.

20. Doughty MJ. Further assessment of gender- and blink pattern-related differences in the spontaneous eyeblink activity in primary gaze in young adult humans. Optom Vis Sci 2002;79:43947.

21. Barbato G, Ficca G, Muscettola G, Fichele M, Beatrice M, Rinaldi F. Diurnal variation in spontaneous eye-blink rate. Psychiatry Res 2000;93:145-51.

22. Hulley SB, Cummings SR, Browner WS, Grady D, Hearst N, Newman TB. Designing Clinical Research. Philadelphia: Lippincott Williams \& Wilkins, 2001:65-91.

23. Morgan P, Efron N, Woods C et al. International Contact Lens Prescribing 2006. Contact Lens Spectrum 2007;22(1):34-8.

24. Schnider C, Terry R, Holden B. Effect of lens design on peripheral corneal desiccation. J Am Optom Assoc 1997;68:163-70. 
25. Müller-Treiber A. 3/9-Uhr-stippen - entstehung und problemmanagement. Die Kontaktlinse 1997;30:5-9.

26. Jones D, Bennett E, Davis L. How to manage peripheral corneal desiccation. Contact Lens Spectrum 1989;4(5):63-6.

27. Douthwaite WA. Contact Lens Optics and Lens Design. Bradford: Butterworth-Heinemann, 2006:69-80.

28. Pointer JS. Eyeblink activity with hydrophilic contact lenses. A concise longitudinal study. Acta Ophthalmol (Copenh) 1988;66:498-504.

29. Collins MJ, Iskander DR, Saunders A, Hook S, Anthony E, Gillon R. Blinking patterns and corneal staining. Eye Contact Lens 2006;32:287-93.

30. Brown M, Chinn S, Fatt I, Harris M. The effect of soft and hard contact lenses on blink rate, amplitude and length. J Am Optom Assoc 1973;44:254-7.

31. Carney LG, Hill RM. Variation in blinking behavior during soft lens wear. Int Contact Lens Clin 1984;11:250-3.

32. York M, Ong J, Robbins JC. Variation in blink rate associated with contact lens wear and task difficulty. Am J Optom Arch Am Acad Optom 1971;48:461-7.

33. Hill RM, Carney LG. The effect of hard lens wear on blinking behavior. Int Contact Lens Clin 1984;11:242-8.

34. Freudenthaler N, Neuf H, Kadner G, Schlote T. Characteristics of spontaneous eyeblink activity during video display terminal use in healthy volunteers. Graefes Arch Clin Exp Ophthalmol 2003;241:914-20.

35. Carney LG, Hill RM. The nature of normal blinking patterns. Acta Ophthalmol (Copenh) 1982;60:427-33.

36. Abelson MB, Holly FJ. A tentative mechanism for inferior punctate keratopathy. Am J Ophthalmol 1977;83:866-9.

37. Nakamori K, Odawara M, Nakajima T, Mizutani T, Tsubota K. Blinking is controlled primarily by ocular surface conditions. Am J Ophthalmol 1997;124:24-30.

38. Yolton DP, Yolton RL, Lopez R, Bogner B, Stevens R, Rao D. The effects of gender and birth control pill use on spontaneous blink rates. J Am Optom Assoc 1994;65:763-70.

39. Schlote T, Kadner G, Freudenthaler N. Marked reduction and distinct patterns of eye blinking in patients with moderately dry eyes during video display terminal use. Graefes Arch Clin Exp Ophthalmol 2004;242:306-12.

40. Collins M, Heron H, Larsen R, Lindner R. Blinking patterns in soft contact lens wearers can be altered with training. Am J Optom Physiol Opt 1987;64:100-3.

41. Yap M. Tear break-up time is related to blink frequency. Acta Ophthalmol (Copenh) 1991;69:92-4.

42. Doane MG. Interactions of eyelids and tears in corneal wetting and the dynamics of the normal human eyeblink. Am J Ophthalmol 1980;89:507-16. 


\section{CHAPTER 6 \\ Summary and Conclusions}


186 CHAPTER 6 


\section{Summary}

With currently available designs, materials and fitting methods, the majority of contact lenses can be fitted and worn successfully. One of the most frequently reported remaining problems, and an important reason for contact lens drop-out, relates to dryness symptoms, which have been reported to possibly affect up to $50 \%$ of all contact lens wearers. While corneal desiccation in soft lens wear (dehydration staining) has gained much attention over the recent years, corneal desiccation in rigid gas permeable lens wear (3- and 9-o'clock staining) has not been widely investigated and cited.

When the characteristics of modern rigid gas permeable lenses are listed, as presented in chapter 1 of this thesis, one could easily be misled and conclude that rigid gas permeable lenses must be the most popular contact lens modality prescribed. But the reality is that, although large variations exist among countries, on average less than one in every ten lens fits around the world is currently with rigid gas permeable lenses. It is generally agreed upon that comfort with this modality is one of the main factors in comparison to soft lens wear, despite the fact that rigid gas permeable lenses are believed to be the safest alternative to glasses for the correction of ametropia. One of the few complications that is typical for, and exclusively seen in, rigid gas permeable lens wear is 3 - and 9-o'clock staining. This condition is the main focus of this thesis, which aims are presented in its first chapter. The condition of 3- and 9-o'clock staining is considered to be the manifestation of corneal desiccation in rigid gas permeable lens wear. The goal of this thesis is to analyze 3- and 9-o'clock staining as well as potential mechanisms behind it to better understand the condition and possibly to better manage it.

Chapter 2 of this thesis is an introduction to rigid gas permeable lens fitting. The first section (part 2.1) tries to answer the question: can we improve the comfort of rigid gas permeable lens wear by optimizing the lens fit? Based on the results from our studies it appears that lens wear comfort is better with lenses that have an optimal lens-to-cornea relationship than with lenses that have a suboptimal lens-tocornea relationship. In order to create such an optimal lens-to-cornea relationship, more sophisticated fitting methods than traditionally used techniques based on keratometry measures to fit contact lenses may be required. In our studies it was found that the majority of lens parameter alterations required for lenses that were fitted with traditional methods could have been predicted by using corneal topography data. 
The second section of chapter two (part 2.2) describes how rigid gas permeable lens fitting can be improved from a practitioner's point of view. This section focuses on respecting the shape of the cornea to optimally distribute equal lens pressure over the entire cornea. If the surface area of contact between lens and cornea is maximized, the pressure of the lens is distributed over the largest possible area of the cornea. To respect the shape of the cornea while fitting rigid gas permeable lenses, flattening of the cornea towards the periphery - which occurs in the majority of corneas - should be taken into account. Since the amount of flattening varies among individuals, it should be assessed separately in every cornea to be of clinical use.

Another important factor of respecting the shape of the cornea is dealing with corneal astigmatism. In doing so, the same principle applies as in dealing with corneal flattening towards the periphery: the aim should be to evenly distribute lens pressure in all meridians of the cornea. Non-toric lenses on astigmatic corneas will create unevenly distributed pressure in particular regions of the corneal surface. To adjust for this, full back surface toric rigid gas permeable lenses can be fitted if substantial amounts of corneal astigmatism are present, especially if the astigmatism stretches out across the entire cornea (limbus-to-limbus corneal astigmatism). Practitioners can use peripheral back surface toric lens designs on corneas with moderate levels of astigmatism and on spherical (or near-spherical) corneas that exhibit an increasing amount of astigmatism towards the periphery.

Chapter 3 focuses on the background and classification of corneal desiccation in rigid gas permeable lens wear: part 3.1 of this thesis provides an overview of 3- and 9-o'clock staining and its possible management options derived from the literature. Epithelial staining in the nasal and temporal areas of the cornea is a well known condition in rigid gas permeable lens wear, but incidence rates vary greatly, ranging from $25 \%$ to $90 \%$ with different levels of clinical significance reported. One of the reasons for this large variation may be that there is no uniform way of classifying the condition. The clinical presentation of 3- and 9-o'clock staining can vary from transient superficial epithelial stippling to deep corneal defects (with stromal involvement) that stain intensely.

Several grading scales to evaluate 3- and 9-o'clock staining are available, but these are all descriptive in nature. Image grading scales have been found to have a better repeatability than descriptive scales. Image grading scales for general corneal staining do exist, but not specifically for 3- and 9-o'clock staining. For this thesis, an image 3- and 9-o'clock staining grading scale was developed and is presented in part 3.2. The scale was developed based on available descriptive grading scales for 
3- and 9-o'clock staining, general image grading scales for corneal staining and the clinical input of a large group of Dutch contact lens practitioners. The major advantage of using photographic images over pictorial scales is that they represent the true clinical picture. However, when developing photographic scales it is a challenge to find a picture that exactly represents the desired grade. Furthermore, differences in anatomy among eyes, lighting conditions and other observational variables hamper the clinical assessment and interpretation of the photographic grades. All of these disadvantages are overcome by pictorial scales in which the condition of interest is sketched at different levels of severity. This option obviously lacks a level of realism, which might be a disadvantage when used in a clinical setting. To benefit from the advantages both systems have to offer, a combination of a background photograph and an overlaid pictorial representation of staining was used for the development of this scale (see back cover of the thesis). The developed 3and 9-o'clock staining image grading scale proved to be linear in nature and therefore it is considered appropriate for practitioners to interpolate between scale images and to use small incremental steps to increase discrimination when grading this condition.

Chapter 4 of this thesis presents the results of clinical studies evaluating 3- and 9o'clock staining in existing rigid gas permeable lens wearers (both with and without 3- and 9-o'clock corneal staining), in order to better understand the condition and its etiology. The first part (part 4.1) evaluates the relationship between signs and symptoms (bulbar hyperemia, conjunctival staining and lens comfort) and 3- and 9-o'clock corneal staining. With regard to the signs related to 3- and 9-o'clock staining, bulbar hyperemia showed a low correlation with corneal staining, but eyes with conjunctival staining did show more corneal staining than eyes without conjunctival staining. No correlation between staining in the nasal and in the temporal parts of the cornea was found in subjects with substantial 3- and 9-o'clock staining. No correlation was found between visual analogue scale (VAS) comfort scores and corneal staining. Symptomatic subjects did show more corneal staining than asymptomatic subjects if substantial 3- and 9-o'clock staining was present.

It is clear that managing 3- and 9-o'clock staining in clinical practice is not easy and can be a frustrating task. Management of the condition is not straightforward, and often it is not possible to resolve the problem completely. Although there are several options suggested in the literature, not many of the publications refer to evidence-based studies. Based on an evaluation of all available information on the subject, including clinical and anecdotal publications, eight variables could be distilled for the management of the condition (part 3.1 of this thesis). Five of these relate to lens parameters, including lens edge lift, back optic zone radius (BOZR), 
back surface geometry, lens diameter and lens edge thickness/shape. Three other factors are related to lens performance, including centration of the lens, lens movement and surface wettability. But there is controversy regarding how to adjust many of these variables with respect to both the direction and the degree of the adjustment. This controversy also concerns the most important (most frequently mentioned) variables: edge lift, BOZR and lens diameter. Part 4.2 of this thesis reports on the investigation of the mentioned variables in existing lens wearers. It was found that flat lens fits, interpalpebral lens fits and large lenses could be a risk factor for the development of 3-and 9-o'clock staining. Based on this, it seems that avoiding flat fitting lenses can be beneficial in managing the condition. However; flat fitted lenses can also create a lid attachment fit, which in turn could be beneficial as well. The same is true for corneal coverage: reducing the lens diameter seems recommended to reduce 3- and 9-o'clock staining based on the results of our studies. But larger lenses can also lead to a lid attachment fit, which again could be beneficial. In other words, these variables cannot be considered as individual factors. But it does explain many of the contradictions found in the literature on how to manage 3- and 9-o'clock staining. The type of lens fit (lid attachment and interpalpebral fit) emerges as the source of many of the controversies and is essential to take into account when managing 3 - and 9-o'clock staining.

To further analyze the possible mechanisms behind the development of 3- and 9o'clock staining, the role of tear volume (tear meniscus height, TMH) and eyeblinking was analyzed and is described in chapter 5. The results, as presented in part 5.1, show that the TMH in rigid gas permeable lens wearers was statistically significantly lower than the TMH in non-lens wearers. This could in theory leave the remaining exposed ocular surface in rigid gas permeable lens wearers with a thinner tear layer, which may give rise to shorter tear break-up-times and may consequently lead to corneal desiccation. In our studies, subjects with substantial corneal staining showed slightly lower TMH values than subjects without substantial corneal staining, but this was not found to be statistically significant.

Large rated lenses demonstrated slightly lower TMH values compared to optimal and small rated lenses, but again this was not statistically significant and no difference at all in TMH between flat lens fits versus optimal/suboptimal and steep lens fits was found in our studies. Based on these findings, it may be suggested that $\mathrm{TMH}$, and therefore possibly tear volume, is influenced by wearing rigid gas permeable lenses. But the changes caused by individual lens parameters are either not substantially different or the method of investigating this is not sensitive enough to pick up the subtle differences that may occur. 
The goal of the study presented in part $\mathbf{5 . 2}$ was to evaluate whether there is a difference in frequency and in completeness of eyeblinks (type of eyeblink) in rigid gas permeable lens wearers experiencing 3- and 9-o'clock staining compared to rigid gas permeable lens wearers without substantial 3- and 9-o'clock staining and to non-lens wearers. No difference in overall eyeblink frequency was found among these three groups. However, substantial differences in different types of eyeblink frequency were found. Fewer complete eyeblinks, more incomplete eyeblinks and more eyeblink attempts were observed in subjects with 3- and 9-o'clock corneal staining compared to rigid gas permeable lens wearers without substantial 3- and 9o'clock staining and compared to non-lens wearers.

With regard to contact lens related variables, fewer complete eyeblinks and more eyeblink attempts were found in interpalpebral lens fits than in lid attachment fits. Also, fewer complete eyeblinks and more incomplete eyeblinks were found with lenses rated as large or optimal in size than with smaller rated lenses. No evidence for an alteration in eyeblink frequency was found for flat versus steep lens fit.

\section{Conclusions}

Based on the studies presented in this thesis, a number of recommendations can be made regarding the clinical implications and some statements can be made regarding the possible mechanisms involved in the development of 3- and 9-o'clock staining, which will be highlighted here.

\section{Clinical Implications}

Signs \& Symptoms

When analyzing comfort in subjects experiencing 3- and 9-o'clock staining, it appears that simply registering the presence or absence of ocular symptoms is a better predictor of 3- and 9-o'clock staining than using VAS comfort scores. But generally, low correlations between corneal staining and lens wear comfort were found, which seems to stress the need for routine contact lens examinations for all rigid gas permeable contact lens wearers.

Practitioners are advised, because of the low correlation between bulbar hyperemia and corneal staining, to be hesitant to use the amount of bulbar hyperemia for prediction of the severity of 3- and 9-o'clock staining. Observation of conjunctival staining could potentially be a useful tool in clinical practice to identify patients at risk for 3-and 9-o'clock staining. 
The special designed grading scale for 3- and 9-o'clock staining may be helpful to practitioners in order to rate, observe and assess the severity of the condition. Because of the linear nature of the scale it is considered appropriate to interpolate between scale images and to use small incremental steps to increase discrimination when grading the condition. It seems advisable that practitioners evaluate both the nasal and temporal side of the cornea when assessing 3- and 9-o'clock staining since the correlation between these two was found to be low in subjects with substantial 3-and 9-o'clock staining.

\section{Lens Fitting}

It was found that flat lens fits, interpalpebral lens fits and large rated lenses were related to higher levels of 3- and 9-o'clock staining. As straightforward as this may seem with regards to management options, some controversy remains. Avoiding flat fitting lenses can be beneficial in managing the condition, but flat fitted lenses can also create a lid attachment fit, which in turn could be beneficial as well. For lens diameter the same controversy was found: smaller lenses showed less 3- and 9o'clock staining in our studies, but larger lenses also can result in a lid attachment fit, which again could be beneficial.

From these findings, two strategies can be distilled for practitioners to manage 3and 9-o'clock staining: to minimize the effect of the lens on the tear film, and at the same time to minimize the effect of the lens on the eyelids. To minimize the effect of the lens on the tear film, the aim should be to respect the shape of the cornea maximally in order to create an optimal lens-to-cornea relationship. Optimal in this regard means an evenly distributed tear film, avoiding large edge clearances or tear menisci around the lens. In classical terms of lens fitting this would mean to aim at lowering the edge clearance of the lens and to decrease lens size.

But at the same time it is apparent to minimize the effect of the lens on the eyelids. At one hand this can be achieved by aiming at the same principle as with limiting the effect on the tear film: lowering the edge clearance and decreasing lens size to minimize the interaction of the upper eyelid with the lens edge to promote proper eyeblinking. Corneal topography to image corneal shape can be helpful in creating lenses that minimize interference with both the tear film and the eyelids. But at the other hand, it was shown that reducing the effect of the lens on the eyelids can also be effectively achieved by aiming at a lid attachment fit, typically by increasing the edge clearance and/or lens size. It should be kept in mind though that lid attachment fits may have some clinical disadvantages, e.g. side effects. Lid attachment fits are associated with unwanted corneal changes (corneal warpage) because these lenses are often decentered on the cornea. This should be monitored carefully by 
the contact lens practitioner on regular follow up visits using corneal topography. Visual performance in lid attached lenses should be monitored as well in clinical practice, since visual disturbances can occur under dim light conditions, resulting in glare and flare because the lens boundaries under those circumstances may be situated within the pupil margin.

One possible solution that has not been brought forward so far is the option to fit a hydrogel lens to solve the problem. This mode of lens wear is not without its own particular problems and corneal desiccation may become manifest in hydrogel lenses as dehydration staining, but the specific problem of 3- and 9-o'clock staining will be solved. In other words, if substantial 3- and 9-o'clock staining is present and is not easy to be solved, hydrogel lenses may be a good alternative. However, many rigid gas permeable lens wearers use this modality for a reason (see chapter one of this thesis). Often the reason is vision related: rigid gas permeable lenses for irregular corneas or bi- and multifocal contact lenses for presbyopes for instance can simply not be replaced by other lens modalities, and hydrogel lenses are not always an option.

In addition, tear supplements and eyeblink exercises have been suggested to remedy 3- and 9-o'clock staining. These factors were not investigated in this thesis. These options may help alleviate the symptoms associated with 3- and 9-o'clock staining. But if they do, they seem to only have a temporary effect and do not contribute to solving the cause of the problem.

\section{Mechanisms behind 3- and 9-O'Clock Staining}

Three main mechanisms have been proposed for the etiology of 3-and 9-o'clock staining: the eyeblink theory, the tear volume theory and the bridge theory. In addition, some have mentioned mechanical forces as a possible mechanism in which with-the-rule corneal toricity is considered as a risk factor.

\section{The Eyeblink Theory}

The eyeblink theory states that rigid gas permeable lens wear could lead to an altered eyeblink frequency, possibly leading to 3- and 9-o'clock staining caused by an increase in interaction between the upper eyelid and the edge of the lens. Because of this, eyeblink exercises are sometimes promoted by practitioners to manage the condition. The hypothesis regarding the eyeblink alteration was confirmed by the results of our studies: the frequency of partial eyeblinks was higher in rigid gas permeable lens wear, and especially so in subjects experiencing substantial 3- and 9o'clock staining. The findings that more 3- and 9-o'clock staining was seen in interpalpebral lens fits than in lid attachment fits and that more partial eyeblinks 
were found in interpalpebral lens fits than in lid attachment fits also seem to point directly to the eyeblinking theory. In addition, the quality of the eyeblink also varied in relation to lens size: eyes with larger lenses showed a higher level of partial eyeblinks, which may also interfere with eyeblink behavior.

Based on these findings it appears that the quality of eyeblinks is altered during rigid gas permeable lens wear, which may be linked to the development of 3- and 9-o'clock corneal staining. Some individual lens variables appear to interfere with the completeness of eyeblinks. If so, it seems advisable to minimize the interaction of the upper eyelid with the lens edge when fitting rigid gas permeable lenses to promote proper eyeblinking. However, flat lens fits did not demonstrate an effect on eyeblink behaviour compared to other lens fits, which is not in line with this theory. But it should be kept in mind that it is difficult to analyze the effect of contact lens related variables (such as lens fit) if they are present in one eye only, while eyeblink frequency is a variable that is measured for both eyes together. Future, prospective studies looking at this effect are needed to further analyse the effect of lens fit on eyeblink quality.

\section{The Tear Volume Theory}

With regard to the tear volume theory, or tear meniscus theory, it has been hypothesized that rigid gas permeable lenses attract tears to the area underneath the periphery of the lens, which may cause thinner tear layers and possibly corneal desiccation in the nasal and temporal portions of the exposed ocular surface. The results of our studies on TMH partly support this theory: statistically significantly lower TMH values were found in rigid gas permeable lens wearers than in non-lens wearers. This unique finding has not been reported before to the best of our knowledge.

Based on the analysis of lens variables in our studies, flat lens fits and large diameter lenses were found to be risk factors for the development of 3- and 9-o'clock staining which seems to be in line with the tear volume theory. Flat lens fits may be expected to attract more tear volume to the periphery of the lens than would optimal and steep lens fits, as could larger diameter lenses compared to smaller lenses. But the TMH values found in our studies were not different for the sequential lens fit groups. And no statistically significant differences in TMH between lens diameter groups were found. In addition, no difference in TMH between rigid gas permeable lens wearers with substantial 3- and 9-o'clock staining and those with minimal 3- and 9-o'clock staining was found. Either there is no relationship between these variables, or the method of measuring TMH is not sensitive enough to pick up the potentially small differences in TMH. It should be kept in mind that 
analysis of lens fit is complicated by the effect of the type of lens fit (interpalpebral versus lid attachment) as was shown in this thesis.

In conclusion: rigid gas permeable lens wear does seem to have an effect on TMH and possibly on the available amount of tear volume on the exposed ocular surface, thus supporting the tear volume theory. With regards to the relationship between $\mathrm{TMH}$ and individual lens variables, questions still remain.

\section{The Bridge Theory}

This theory states that due to a bridge that is formed by the upper eyelid between the edge of the lens and the cornea during eyeblinking, a gap is created under the eyelid and corneal mucin coverage of the area under the 'bridge' may be inadequate. Unfortunately, measurement techniques for mucin coverage are not readily available and this mechanism was not directly investigated in our studies.

Indirectly, the finding presented in this thesis that flat fitting lenses caused more 3and 9-o'clock staining could support this theory: flat lens fits will increase the bridge effect and potentially reduce mucin spreading over the cornea. Lenses with more corneal coverage (large diameter lenses) were found to result in more corneal staining as well, but it is less obvious than with flat lens fits if and how this relates to the bridge theory. At one hand do larger lenses cover more of the affected 3- and 9-o'clock positions on the cornea which prevents exposure of these areas of the cornea: the staining is always adjacent to the lens, never under it. At the other hand, smaller lenses do leave more of the cornea exposed to the palpebral eyelid, facilitating better mucin spreading over the corneal periphery and also may small lenses have smaller edge clearances thus decreasing the bridge effect and the risk of 3- and 9-o'clock staining. Therefore, this finding of larger diameter lenses being a risk factor for 3- and 9-o'clock staining could potentially be supporting the bridge theory.

No relationship between lens movement and corneal staining was found, which may have been expected if the bridge theory is followed, since decreased movement of the lens is believed to potentially inhibit mucin spreading over the cornea.

The eyeblink findings do not seem to contribute directly to the bridge effect. It could only have a secondary, amplifying effect on the bridge mechanism in the development of 3- and 9-o'clock staining: if the bridge effect leads to a decrease in tear film stability nasally and temporally, this could theoretically be exaggerated by a decrease in eyeblink quality. 
Generally; more controlled manipulation of the post lens tear film thickness to create the desired edge clearance may in theory help to reduce the bridge theory effect, but this mechanism could not be investigated directly.

\section{The Mechanical Theory}

No evidence was found in our studies to support the mechanical theory in which with-the-rule corneal astigmatism is considered as a risk factor for 3- and 9-o'clock staining. No correlation between the amount of corneal staining and amount or type of corneal astigmatism was found. Central keratometry values were used to establish the amount and type of corneal astigmatism and it has been shown with the use of corneal topographers that corneal astigmatism can increase or decrease towards the periphery, and also that the corneal astigmatism axis may vary towards the periphery. In other words, there may be a relationship between overall corneal astigmatism and 3- and 9-o'clock staining; however central keratometry alone is inadequate to characterize this.

In summary regarding the possible mechanisms behind 3- and 9-o'clock staining: the findings presented in this thesis seem to point to more than one possible etiology for 3- and 9-o'clock staining, possibly amplifying each other. But the most widely supported theory appears to be the eyeblink theory, followed by the tear volume theory. The bridging effect is another possibility, although this variable could not be tested directly. No evidence was found to support the mechanical theory.

\section{Closing Remarks}

Although the results of the studies presented in this thesis are not conclusive on the etiology of 3- and 9-o'clock staining, it appears that most findings point in a certain direction when it comes to managing the condition. It can be concluded that the aim when managing 3- and 9-o'clock staining should be to limit the effect of the lens on both the tear film and the eyelids. Practical guidelines have been provided to practitioners to manually achieve this with classical fitting techniques. But to do so optimally, accurate measurement of corneal shape with corneal topographers seems unavoidable to create better lens shapes. This can help limit the effect on both tear film and eyelids. Custom made contact lenses based on corneal topography could be a helpful tool in managing 3- and 9-o'clock staining, and seems to be the most logical way forward for the future of the rigid gas permeable lens. The technology to design and produce these custom made contact lenses is available. Maybe it is time to start using this technology on a larger scale in clinical practice, and to move away from the traditional fitting techniques. 


\section{Samenvatting en Conclusies}

\section{Samenvatting}

Met de huidige beschikbare lensontwerpen, materialen en aanpasmethoden kunnen contactlenzen in de meeste gevallen succesvol worden aangepast en gedragen. Als er problemen zijn bij het dragen van contactlenzen is een van de meest frequent gerapporteerde oorzaken gerelateerd aan droge ogen. Mogelijk dat de helft van alle lensdragers hier direct of indirect mee te maken heeft. Terwijl de laatste jaren veel aandacht is uitgegaan naar problemen met droge ogen bij zachte contactlenzen (dehydratie staining), is weinig onderzoek gedaan naar problemen met droge ogen bij vormstabiele lensdragers (3- en 9-uur staining).

Als de voordelen van vormstabiele contactlenzen ten opzichte van zachte contactlenzen worden opgesomd (hoofdstuk 1), zou men op basis van deze theoretische gronden misleid kunnen worden en veronderstellen dat vormstabiele contactlenzen de meest populaire vorm van contactlenscorrectie moet zijn. De realiteit is dat slechts één op elke tien lenspassingen wereldwijd met vormstabiele contactlenzen is, al wisselt dit sterk per regio. Aangenomen wordt dat de belangrijkste reden voor de populariteit van zachte contactlenzen ten opzichte van vormstabiele contactlenzen het draagcomfort is. Dit lijkt belangrijker dan bijvoorbeeld de veiligheid van het lensdragen: aangetoond is dat vormstabiele contactlenzen minder kans op complicaties veroorzaken dan zachte contactlenzen.

Eén van de weinige complicaties die zich bij vormstabiele contactlenzen voordoet, en die exclusief is voorbehouden aan vormstabiele contactlenzen, is 3- en 9-uur corneale staining. Dit wordt gezien als de manifestatie van droge ogen bij vormstabiele contactlenzen, en uit zich in beschadigde epitheelcellen in de nasale en temporale (3- en 9-uur) gebieden van de cornea. Deze contactlensgerelateerde complicatie staat centraal in dit proefschrift. De doelen hiervan worden in het eerste hoofdstuk van dit proefschift uiteengezet. Het proefschrift richt zich allereerst op de subjectieve en objectieve tekenen van de aandoening, en vervolgens op de mechanismen achter de mogelijke oorzaak ervan. Het streven is 3- en 9-uur staining en zijn mogelijke oorzaak beter te doorgronden, om zodoende inzichten te verkrijgen die kunnen leiden tot het beter managen van de aandoening in de praktijk. 
Allereerst wordt in hoofdstuk 2 van dit proefschrift een introductie in het aanpassen van vormstabiele contactlenzen weergegeven. Het eerste deel (deel 2.1) probeert een antwoord te vinden op de vraag of het draagcomfort van vormstabiele contactlenzen verbeterd kan worden door de aanpassing van de lens te optimaliseren. Op basis van de resultaten van onze studie blijkt dat het draagcomfort van vormstabiele contactlenzen die de cornea optimaal respecteren (en zodoende een optimale lenspassing hebben) beter is dan van vormstabiele contactlenzen met een suboptimale lenspassing. Om een optimale lenspassing te creëren is het aan te raden om gebruik te maken van nieuwere aanpastechnieken op basis van corneatopografie en niet van de traditionele aanpastechnieken op basis van centrale keratometrie uit te gaan. In onze studie werd aangetoond dat de meerderheid van de veranderingen die nodig was om bij traditioneel aangepaste contactlenzen een optimale lenspassing te creëren, vooraf voorspeld had kunnen worden als gebruik was gemaakt van corneatopografie data.

Het tweede deel van dit hoofdstuk (deel 2.2) beschrijft hoe de aanpassing van vormstabiele contactlenzen in de praktijk kan worden verbeterd. Het richt zich op het optimaal respecteren van de vorm van de cornea, om een evenredige drukverdeling te krijgen van de lens op het corneaoppervlak. Om dit te bereiken zal de afvlakking die plaatsvindt van centrum naar periferie (de excentriciteit) bij een gemiddelde cornea in aanmerking genomen moeten worden. Maar omdat de excentriciteit wisselt per oog, moet dit bij elke cornea afzonderlijk in kaart worden gebracht om van praktische waarde te kunnen zijn.

Een andere factor die een belangrijke rol speelt bij het respecteren van de vorm van de cornea aangaande het aanpassen van vormstabiele contactlenzen, is het corneaastigmatisme. Hiervoor geldt hetzelfde principe als bij cornea-excentriciteit. Het streven is om een gelijke drukverdeling te creëren over het corneaoppervlak, waarbij in dit geval de verschillen in corneavorm per meridiaan gerespecteerd moeten worden. Om dit te bereiken kunnen volledig binnentorische contactlenzen worden aangepast als er forse graden cornea-astigmatisme aanwezig zijn, en met name als het cornea-astigmatisme zich uitstrekt over de gehele cornea (van limbus tot limbus). Perifeer-torische contactlenzen kunnen worden aangepast bij cornea's met een gering cornea-astigmatisme en bij cornea's die centraal sferisch zijn (of bijna sferisch) maar waarbij het astigmatisme toeneemt naar de periferie.

Hoofdstuk 3 richt zich op de achtergronden en classificatie van 3- en 9-uur staining. Deel 3.1 van dit proefschrift bevat een overzichtsartikel betreffende 3- en 9uur staining, wat ingaat op de terminologie, de achtergronden en de mogelijke oor- 
zaak van de aandoening. Tevens wordt een opsomming gegeven van de opties die in de literatuur worden aangedragen om het probleem te beter te managen.

Epitheliale staining in de nasale en temporale delen van de cornea is een veel voorkomend fenomeen bij vormstabiele lensdragers, maar de gevonden incidentie ervan varieert sterk in de litaratuur van $25 \%$ tot $90 \%$. Eén van de redenen waarom de variatie zo groot zou kunnen zijn, is dat er geen uniforme manier is om 3- en 9-uur staining te graderen. Bij 3- en 9-uur staining kan de mate van staining variëren van zeer oppervlakkige staining tot een diep corneaal defect met intense staining, waarbij het stroma betrokken kan zijn.

Diverse graderingsschalen zijn beschikbaar om 3- en 9-uur staining te graderen, maar deze zijn allen beschrijvend van karakter. Er zijn wel visuele graderingsschalen voor corneale staining beschikbaar op basis van klinische beelden van de aandoening. Maar deze zijn voor algemene corneale staining ontworpen en niet specifiek voor 3- en 9-uur staining. Voor dit proefschrift werd een speciale 5-stap visuele graderingsschaal ontworpen, welke is weergegeven in deel 3.2. De graderingsschaal is gebaseerd op de beschikbare beschrijvende 3- en 9-uur staining schalen, en op basis van de algemene visuele graderingsschalen voor corneale staining. Tevens werd de inbreng van een grote groep contactlensspecialisten en optometristen in Nederland gebruikt om de graderingsschaal verder te ontwikkelen en te verfijnen. Voor het ontwerpen van een dergelijke graderingsschaal kunnen praktijkfoto's worden gebruikt, waarvan het voordeel is dat het de praktijksituatie goed representeert. Echter, indien een visuele graderingsschaal wordt ontwikkeld, is het niet altijd eenvoudig om exact dát beeld te vinden dat de klinische situatie op een bepaald moment het beste weergeeft. Tevens zijn er anatomische verschillen tussen ogen, verschillen in lichtcondities en andere observatiebeperkingen die een goede weergave bemoeilijken. Al deze beperkingen worden weggenomen als wordt overgegaan op graderingsschalen die geanimeerd zijn, zodat exact het juiste gewicht aan een bepaalde fase van de conditie kan worden gehangen en visueel kan worden gemaakt. Het voornaamste nadeel hiervan is uiteraard dat het een niet-realistische weergave van de werkelijkheid geeft.

Om gebruik te maken van de voordelen die beide soorten graderingsschalen kunnen bieden, is een combinatie van een achtergrondfoto van de cornea met fluoresceïnebeeld van een vormstabiele contactlens gebruikt waarop vervolgens softwarematig de hoeveelheid staining werd angebracht (zie achterkant van dit proefschrift). Het is aangetoond dat discriminatie van het graderen toeneemt als er gegradeerd wordt in kleine stappen (tot op decimalen nauwkeurig), maar hiervoor moet een graderingsschaal wel lineair van aard zijn. Er werd aangetoond in deze 
studie dat de ontwikkelde 3- en 9-uur staining graderingsschaal een lineair karakter heeft, welke daardoor gebruikt kan worden om tussen de hele stappen van de schaal te interpoleren.

Hoofdstuk 4 geeft een overzicht van de klinische studies naar 3- en 9-uur staining bij bestaande lensdragers, om inzicht te krijgen in het ontstaan van de aandoening en om mogelijke opties te distilleren om het probleem beter te managen. Het eerste deel (deel 4.1) analyseert de relatie tussen de subjectieve en objectieve karakteristieken van 3- en 9-uur staining, waaronder met name bulbaire hyperemie, conjunctivale staining en draagcomfort. De correlatie tussen bulbaire hyperemie en corneale staining was laag bij lensdragers met substantiële 3- en 9-uur staining. Maar ogen met conjunctivale staining lieten wel meer corneale staining zien dan ogen zonder conjunctivale staining. Nasale en temporale staining waren niet gecorreleerd, en er werd ook geen correlatie tussen draagcomfort en VAS (visual analogue scale) comfort scores gevonden. Symptomatische lensdragers vertoonden meer corneale staining dan asymptomatische lensdragers in de groep met substantiële 3- en 9-uur staining.

Het managen van 3- en 9-uur staining in de praktijk is geen simpele, en vaak een frustrerende taak. Soms is het onmogelijk het probleem volledig te verhelpen. De therapeutische oplossingen voor deze contactlenscomplicatie die worden geboden in de literatuur zijn vaak in strijd met elkaar. En alhoewel er diverse oplossingen worden aangedragen in de internationale literatuur, zijn niet veel van deze factoren gebaseerd op 'evidence-based' studies. Op basis van een evaluatie van alle beschikbare literatuur op dit gebied konden acht mogelijke variabelen worden gedistilleerd die belangrijk kunnen zijn bij het managen van 3- en 9-uur staining. Vijf van deze acht variabelen zijn lensparameters: lensradius, edge lift, binnengeometrie van de lens, lensdiameter en ten slotte de dikte en vorm van de lensrand. Drie andere variabelen zijn gerelateerd aan het gedrag van de lens op het oog: lenscentratie, beweging van de lens en de bevochtiging van de lens. Maar er is verdeeldheid in de literatuur wat betreft de mate en het soort van veranderingen die aangebracht moeten worden om de staining te verminderen aangaande veel van deze variabelen. Het betreft hierbij ook de ogenschijnlijk meest belangrijke variabelen: edge lift, lensradius en lensdiameter (deze variabelen worden het vaakst genoemd en/of als belangrijkste aangegeven). Deel 4.2 van dit proefschrift onderzoekt de genoemde parameters bij bestaande lensdragers.

Uit onze studie bleek dat vlakke lensaanpassingen, interpalpebrale lenspassingen en grotere lensdiameters risicofactoren kunnen zijn voor het ontstaan van 3- en 9-uur staining. Op basis hiervan lijkt het aan te raden vlakke lenspassingen te vermijden 
bij 3- en 9-uur staining. Echter: vlakke contactlenzen kunnen ook een lidattachment lenspassing veroorzaken, wat weer een positief effect kan hebben op 3en 9-uur staining. Dezelfde controverse geldt ook voor lensdiameter. Er wordt aangeraden een kleinere lensdiameter te gebruiken om 3- en 9-uur staining te beperken, maar grotere contactlenzen kunnen leiden tot lid-attachment lenspassingen wat ook kan bijdragen aan het verminderen van 3- en 9-uur staining. De voornaamste conclusie is derhalve dat de genoemde variabelen niet als individuele factoren gezien kunnen worden. Dit verklaart wel de controverse in de literatuur met betrekking tot deze variabelen. Het type lenspassing (lid-attached of interpalpebraal) speelt dus een belangrijke rol bij 3- en 9-uur staining, en het effect hierop dient te allen tijde meegewogen te worden indien andere parameters worden aangepast.

Om de mogelijke achterliggende mechanismen betreffende 3- en 9-uur staining verder te onderzoeken werden de rol van het traanfilmvolume (gemeten als de traanmeniscus hoogte, TMH) en het knipperpatroon van vormstabiele lensdragers onderzocht (hoofdstuk 5). Deze onderzoeken lieten zien dat de TMH van vormstabiele lensdragers lager was dan van niet-lensdragers (deel 5.1). In theorie zou een verminderd traanvolume op het oculaire oppervlak kunnen leiden tot een dunnere en minder stabiele traanfilm, mogelijk leidend tot uitdroging van de cornea en 3- en 9-uur staining. Er werd tevens gevonden dat lensdragers met substantiële 3en 9-uur staining een lagere TMH waarde vertoonden dan lensdragers zonder substantiële 3- en 9-uur staining, maar dit was niet statistisch significant. Bij grotere contactlenzen werden iets lagere TMH waarden gevonden dan bij optimale en kleinere diameter contactlenzen, maar ook dit was niet statistisch significant. Geen verschil in TMH werd gevonden voor lenspassing (vlakke versus diepe lenspassingen). Op basis van de bevindingen van deze studie werd geconcludeerd dat TMH, en daarmee mogelijk het traanvolume op het oog, beïnvloed wordt door het dragen van vormstabiele contactlenzen. De hypothetische TMH veranderingen die door invloed van verschillende lensparameters verwacht zouden kunnen worden zijn óf niet substantieel, óf de methode om dit te meten is niet gevoelig genoeg om de mogelijk subtiele veranderingen die aanwezig zijn aantoonbaar te maken.

Het doel van het onderzoek dat gepresenteerd is in deel $\mathbf{5 . 2}$ was om te evalueren of er een verschil is in de frequentie en in de volledigheid van knipperslagen tussen vormstabiele lensdragers (met en zonder 3- en 9-uur staining) en niet-lensdragers. Er werd geen verschil in totale knipperfrequentie gevonden tussen deze groepen. Echter, minder complete knipperslagen, meer incomplete knipperslagen en meer pogingen-tot-knipperslag werden waargenomen bij lensdragers met 3- en 9-uur staining vergeleken met lensdragers zonder 3- en 9-uur staining, en ook dan bij 
niet-lensdragers. In relatie tot individuele contactlensgerelateerde variabelen werd gevonden dat minder complete knipperslagen en meer pogingen-tot-knipperslag werden gezien bij interpalpebrale lenspassingen dan bij lid-attachment lenspassingen. Tevens werden minder complete knipperslagen en meer incomplete knipperslagen gevonden bij lenzen die als groot of normaal werden aangemerkt in vergelijking met contactlenzen die als klein werden aangemerkt. Geen bewijs werd gevonden voor een invloed van de lenspassing (vlakke lenspassing versus diepe lenspassing) op het knippergedrag van vormstabiele lensdragers.

\section{Conclusies}

Op basis van de studies in dit proefschrift kunnen een aantal aanbevelingen worden gedaan met betrekking tot de klinische implicaties en enkele uitspraken worden gedaan met betrekking tot de mogelijke mechanismen die 3- en 9-uur staining kunnen veroorzaken.

\section{Klinische Implicaties}

\section{Subjectieve en Objectieve Bevindingen}

Om het draagcomfort te evalueren van dragers van vormstabiele contactlenzen met 3- en 9-uur staining lijkt het eenvoudigweg registreren van de aan- of afwezigheid van symptomen een betere voorspellende waarde te hebben dan het gebruik van VAS comfort scores. Maar in het algemeen geldt dat er lage correlaties tussen draagcomfort en corneale staining werden gevonden. Dit suggereert dat routinematige contactlenscontroles om 3- en 9-uur staining op te sporen noodzakelijk zijn, daar het draagcomfort geen duidelijke aanwijzing geeft voor de aanwezigheid van de contactlenscomplicatie.

Vanwege de lage correlatie tussen bulbaire hyperemie en corneale staining is het tevens niet aan te raden de hoeveelheid hyperemie mee te laten wegen in het oordeel over de mate van 3- en 9-uur staining. Het observeren van conjunctivale staining daarentegen kan potentieel van toegevoegde waarde zijn in de praktijk voor het analyseren van de ernst en aard van 3- en 9-uur staining.

De ontwikkelde 3- en 9-uur staining graderingsschaal kan een bijdrage leveren aan het observeren en graderen van de aandoening. Vanwege de lineariteit van de schaal kan er tussen de vijf stappen van de schaal geïnterpoleerd worden, zodat het discriminerend vermogen van het graderen toeneemt. Het is van belang zowel de nasale als temporale zijde van de cornea te evalueren, en de maximale hoeveelheid staining te gebruiken voor het inschatten van de omvang van de staining omdat de 
correlatie tussen de mate van nasale en temporale staining laag was in ons onderzoek.

\section{Lenspassing}

Vlakke lenspassingen, interpalpebrale lenspassingen en grotere contactlenzen lijken een verhoogd risico te zijn voor 3- en 9-uur staining. Op basis hiervan lijkt een advies voor de lenspassing eenvoudig, maar toch blijft er ruimte voor controverse en discussie. Want terwijl het streven is om vlakke lenspassingen te voorkomen, kunnen vlakke lenspassingen ook leiden tot lid-attachment lenspassingen welke een positief effect kunnen hebben. Met betrekking tot lensdiameter geldt dezelfde controverse: minder 3- en 9-uur staining werd waargenomen bij kleinere contactlenzen, maar grotere contactlenzen kunnen leiden tot een lid-attachment lenspassing met een mogelijk gunstig effect op 3- en 9-uur staining.

Op basis van het voorgaande kan geconcludeerd worden dat het advies bij 3- en 9uur staining is om de invloed van de lens op de traanfilm alswel op het ooglid zoveel mogelijk te minimaliseren. Om het effect van de lens op de traanfilm zoveel mogelijk te beperken moet ernaar gestreefd worden de vorm van de cornea zoveel mogelijk te respecteren, zodat een optimaal lens-cornea profiel gecreëerd wordt. Optimaal in dit opzicht betekent een gelijkmatige traanfilm tussen lens en cornea, waarbij een grote perifere lift en traanmeniscus rond de lensrand vermeden dienen te worden. In klassieke lensaanpastermen betekent dit doorgaans de perifere lift minimaliseren en de lensdiameter klein houden.

Maar tegelijkertijd moet het streven zijn de invloed van de lens op de oogleden zoveel mogelijk te verminderen om het knippergedrag van de lensdrager zo optimaal mogelijk te houden. Dit kan aan de ene kant bereikt worden door dezelfde strategie te hanteren als bij de invloed van de lens op de traanfilm: de perifere lift verminderen en de diameter verkleinen om de interactie tussen bovenste ooglid en lensrand zoveel mogelijk te beperken. Corneatopografie data die de corneavorm nauwkeurig in kaart brengen kunnen een belangrijke toegevoegde waarde zijn om de invloed van de lens op zowel de traanfilm als op het ooglid te verminderen. Echter, aan de andere kant is duidelijk dat het effect van de lens op het ooglid ook effectief kan worden verminderd door een lid-attachment lenspassing te creëren, waarbij juist een grotere perifere lift en/of een grotere lensdiameter aan te raden zijn. Hierbij moet wel aangemerkt worden dat lid-attachment lenspassingen enkele nadelige gevolgen kunnen hebben voor de lensdrager. Lid-attachment lenspassingen kunnen leiden tot een toename van cornea-irregulariteiten (corneal warpage) omdat de lenzen doorgaans gedecentreerd zijn op de cornea. Dit mogelijke effect moet worden geëvalueerd door middel van corneatopografie tijdens routinecontro- 
les. Tevens kunnen er visuele klachten ontstaan: gedecentreerde lenzen die onder het bovenste ooglid gepositioneerd zitten kunnen leiden tot visusklachten in schemerlicht omdat de lensrand binnen het pupilbereik komt. Dit kan leiden tot storende schitteringen en vertekeningen in dergelijke omstandigheden.

Een alternatieve mogelijkheid die tot nu toe nog niet genoemd is, is de mogelijkheid een zachte lens aan te passen om het probleem van 3- en 9-uur staining het hoofd te bieden. Theoretisch gezien kan dit inderdaad een prima alternatief zijn. Aangezien 3- en 9-uur staining gerelateerd is aan droge ogen, is er geen garantie dat het dragen van hydrofiele contactlenzen probleemloos zal zijn omdat dehydratie staining een veel voorkomende complicatie is bij het dragen van zachte contactlenzen. Echter, het kan ook zeker niet worden uitgesloten dat zachte contactlenzen wel succesvol kunnen zijn, zodat indien substantieel 3- en 9-uur staining aanwezig is dat een chronisch karakter heeft, zachte contactlenzen een goed alternatief kunnen zijn. Echter, veel vormstabiele lensdragers dragen deze lenzen voor een reden. Vaak is die reden gerelateerd aan gezichtsscherpte. Vormstabiele contactlenzen voor de irregulaire cornea, en bi- en multifocale vormstabiele contactlenzen voor presbyopie kunnen bijvoorbeeld simpelweg niet vervangen worden door andere lensmodaliteiten, en zachte contactlenzen zijn derhalve lang niet altijd een optie.

Ook knipperoefeningen en het druppelen van kunstmatig traanvocht zijn mogelijke middelen om 3- en 9-uur staining te verminderen. Deze factoren zijn niet onderzocht in dit proefschrift. Deze opties kunnen een mogelijkheid bieden om de symptomen van 3- en 9-uur staining te verminderen, maar zijn tijdelijk van aard en kunnen niet de oorzaak van het probleem wegnemen.

\section{Mechanismen achter 3- en 9-Uur Staining}

Er zijn drie hoofdtheorieën geïntroduceerd die het ontstaan van het fenomeen 3en 9-uur staining proberen te verklaren: de knipperslag theorie, de traanvolume theorie en de brug theorie. Tevens is er gesuggereerd dat mechanische factoren een rol kunnen spelen bij het ontstaan van 3- en 9-uur staining, waarbij corneaastigmatisme volgens-de-regel als risicofactor wordt aangemerkt.

\section{De Knipperslag Theorie}

Deze theorie gaat ervan uit dat het dragen van vormstabiele contactlenzen het knippergedrag van de lensdrager kan beïnvloeden, door de interactie tussen lensrand en bovenste ooglid tijdens de knipperslag. Knipperoefeningen worden regelmatig geadviseerd in de praktijk in een poging dit effect zoveel mogelijk te minimaliseren. Deze hypothese werd bevestigd door de resultaten van ons onderzoek: de frequentie van onvolledige knipperslagen was hoger bij dragers van vormstabiele 
contactlenzen dan bij niet-lensdragers, en ook werden meer onvolledige knipperslagen gevonden bij lensdragers met 3- en 9-uur staining dan bij diegenen zonder 3- en 9-uur staining. De bevindingen dat meer 3- en 9-uur staining werd waargenomen bij lensdragers met een interpalpebrale lenspassing, en dat meer onvolledige knipperslagen werden waargenomen bij lensdragers met een interpalpebrale lenspassing lijken een directe aanwijzing te zijn in de richting van deze theorie. Ook de bevinding dat lensdragers met grotere lenzen meer onvolledige knipperslagen vertoonden dan lensdragers met normale of kleinere lenzen kan in dezelfde richting wijzen.

Op basis van bovenstaande bevindingen kan gesteld worden dat de kwaliteit van de knipperslag beïnvloed kan worden door het dragen van vormstabiele contactlenzen, met een mogelijke relatie tussen knippergedrag en het ontstaan van 3- en 9-uur staining. Sommige individuele lensvariabelen (in het bijzonder lensdiameter en interpalpebrale lenspassingen) bleken een invloed op de kwaliteit van de knipperslag te hebben en ondersteunen derhalve de knipperslag theorie. Maar, vlakke lenspassingen demonstreerden geen effect op de knipperkwaliteit, wat op basis van theoretische overwegingen wel verwacht zou kunnen worden. Het onderzoeken van lenspassing in relatie tot knippergedrag is echter geen eenvoudige opgave omdat de lenspassing per oog verschillend kan zijn, terwijl de knipperfrequentie voor beide ogen gelijk is. Toekomstige, prospectieve studies zijn nodig om de invloed van lenspassing (voor beide ogen) op de knipperkwaliteit verder te onderzoeken.

\section{De Traanvolume Theorie}

Met betrekking tot de traanvolume- of traanmeniscustheorie wordt verondersteld dat vormstabiele contactlenzen traanvocht onttrekken aan het oculaire oppervlak naar de periferie van de lens, wat kan leiden tot een minder stabiele traanfilm en mogelijk corneale uitdroging in de 3- en 9-uur posities van de cornea. De resultaten van de TMH studie onderschrijven deze theorie ten dele: statistisch significante lagere TMH waarden werden gevonden bij vormstabiele lensdragers in vergelijking met niet-lensdragers. Deze unieke bevinding is tot nu toe niet eerder gerapporteerd.

De bevindingen dat grotere lenzen en vlakke lenspassingen een risicofactor kunnen zijn voor het ontstaan van 3- en 9-uur staining lijkt in lijn met de traanvolume theorie. Vlakke lenspassingen kunnen meer traanvocht onttrekken aan het oculaire oppervlak, en hetzelfde geldt voor grotere lenzen. Maar een relatie tussen lenspassingen en TMH werd niet gevonden in ons onderzoek. Geen statistisch significant verschil werd gevonden tussen verschillende lensdiameters en de TMH. Ook werd er geen statistisch significant verschil in TMH gevonden tussen lensdragers met 3- 
en 9-uur staining en diegenen zonder 3- en 9-uur staining. Er is óf geen verschil in $\mathrm{TMH}$ voor de gemeten variabelen, óf de verschillen zijn te klein en/of de methode om dit te meten te grof om de potentieel aanwezige kleine verschillen te kunnen meten. Hierbij moet aangetekend worden dat het effect van het type lenspassing (interpalpebraal versus lid-attached) een complicerende factor is bij het analyseren van deze variabelen, zoals eerder in dit proefschrift is aangetoond.

Concluderend: de TMH bij vormstabiele lensdragers was lager dan bij nietlensdragers, wat een directe ondersteuning van de traanvolume theorie lijkt te zijn. Maar met betrekking tot individuele lensvariabelen blijft de relatie onduidelijk.

\section{De Brug Theorie}

Deze theorie stelt dat doordat er een brug wordt gevormd door het bovenste ooglid, met aan de ene kant van de brug de afstaande lensrand en aan de andere kant de cornea, er een ruimte ontstaat onder het ooglid. Hierdoor is de verspreiding van mucine over de corneale epitheelcellen onder de 'brug' suboptimaal, en kan er 3en 9-uur staining ontstaan. Meettechnieken voor mucine spreiding zijn echter niet voorhanden en deze theorie kon derhalve niet direct getest worden in onze onderzoeken.

Indirect zou het gegeven dat vlakkere lenzen een verhoogde kans op 3- en 9-uur staining geven gezien kunnen worden als een ondersteuning van deze theorie. Immers: als de lensrand meer afstaat, neemt het brug effect toe. Grotere lenzen bleken ook een risicofactor te zijn voor het onstaan van 3- en 9-uur staining, maar het is niet meteen duidelijk hoe lensdiameter zich verhoudt tot de brug theorie. In de praktijk worden vaak juist grotere diameter lenzen aangepast, omdat dit de blootstelling van het oculaire oppervlak verkleint, en het gebied waar uitdroging kan plaatsvinden verkleint (3- en 9-uur staining bevindt zich altijd naast de lens, nooit onder de lens). Aan de andere kant ontstaat er bij kleinere lenzen meer blootstelling van corneale epitheelcellen aan de palpebrale conjunctiva, wat voor een betere mucine spreiding kan zorgen. Kleinere lenzen hebben doorgaans een dunnere lensrand en perifere lift, wat ook een gunstig effect kan hebben op het brug effect. Derhalve zou deze bevinding, dat grotere lenzen een risicofactor zijn voor 3- en 9-uur staining, mogelijk aan kunnen sluiten bij de brug theorie.

Er werd geen relatie gevonden tussen beweging van de lens en 3- en 9-uur staining, terwijl op basis van de brug theorie een betere beweging van de lens, leidend tot een betere mucine spreiding en daarmee tot een vermindering in 3- en 9-uur staining, aannemelijk zou zijn geweest. 
De resultaten van de knipperslag studie laten een afname van de kwaliteit van de knipperslag zien, dus meer onvolledige knipperslagen, bij vormstabiele lensdragers en bij mensen met 3- en 9-uur staining. Alhoewel dit op zichzelf geen directe invloed op het brug effect heeft, kan het mogelijk indirect een bijdrage leveren aan het ontstaan van 3- en 9-uur staining, door versterking van het effect dat ontstaat door het brug effect.

Samenvattend: een betere controle van het traanprofiel tussen lens en cornea met een gereduceerde perifere lift zou in theorie een positief effect op het brug effect kunnen hebben. De bevinding dat vlakke lenzen een risicofactor zijn voor 3- en 9uur staining zou hiervan indirect een bevestiging kunnen zijn, maar het mucine spreiding mechanisme kon niet direct worden gemeten in onze studies.

\title{
De Mechanische Theorie
}

Geen bewijs werd gevonden voor de mechanische theorie, waarbij astigmatisme volgens-de-regel als risicofactor wordt gezien. Er werd geen correlatie gevonden tussen de hoeveelheid staining en de mate en soort cornea-astigmatisme. Hierbij werd gebruikt gemaakt van centrale keratometriewaarden, en het is aangetoond dat de mate van astigmatisme, en de richting van het astigmatisme, kunnen toenemen of afnemen naar de periferie van de cornea. Met andere woorden, het is mogelijk dat er een relatie tussen 3- en 9-uur staining en totaal cornea-astigmatisme bestaat, maar centrale keratometriewaarden alleen bleken onvolledig in staat deze relatie aan te tonen.

Samenvattend met betrekking tot de mogelijke mechanismen achter het onstaan van 3- en 9-uur staining: de bevindingen in dit proefschrift lijken in de richting van meer dan één oorzaak te wijzen. De resultaten van onze studies lijken de knipperslag theorie het meest te ondersteunen, gevolgd in tweede instantie door de traanvolume theorie. Het brug effect is een derde optie, die deels werd ondersteund maar niet direct kon worden onderzocht. Verschillende mechanismen kunnen mogelijk tegelijkertijd plaatsvinden en elkaar eventueel versterken. De onderzoeken vonden geen bewijs voor de mechanische theorie.

\begin{abstract}
Afsluitend
Alhoewel de gepresenteerde resultaten in dit proefschrift geen definitief uitsluitsel geven betreffende een eenduidige oorzaak van 3- en 9-uur staining, kan wel gesteld worden dat de uitkomsten in een bepaalde richting wijzen als het gaat om het beter managen van de aandoening. De conclusie is dat het beste gestreefd kan worden naar het beperken van het effect van de lens op zowel de traanfilm als de oogleden. Praktische adviezen om dit te bereiken met de klassieke aanpastechnieken zijn be-
\end{abstract}


schreven in dit proefschrift. Echter, om een optimaal effect te bereiken lijkt het onoverkomelijk om gedetailleerde informatie over de corneavorm te verkrijgen. Corneatopografie kan bijdragen aan de strategie om het effect van de lens op zowel de traanfilm als de oogleden te minimaliseren. Custom-made contactlenzen op basis van corneatopografie kunnen een bruikbaar hulpmiddel zijn bij het voorkomen van 3- en 9-uur staining, en lijken tevens de meest logische weg voor de toekomst van de vormstabiele contactlens. De technologie om deze contactlenzen te ontwerpen en fabriceren is inmiddels beschikbaar. Misschien wordt het tijd om in plaats van de nog veelgebruikte klassieke aanpastechnieken, de op basis van de nieuwste technologie gebaseerde technieken op grotere schaal toe te gaan passen in de dagelijkse contactlenspraktijk. 


\section{Abbreviations \& Terms}

3-and 9-o'clock staining corneal staining in the three- and nine o'clock positions of the cornea in rigid gas permeable contact lens wear

AS

BOZR

BUT

epithelial staining

$\mathrm{Dk}$

$\mathrm{Dk} / \mathrm{t}$

DW

edge clearance

edge lift

$e$-value

EW

FDA

fluorescein

GP

hard lenses

HEMA

hydrogel lenses

IEBI

interpalpebral lens fit

lid attachment lens fit

k-reading

MC

MST

NIBUT

NRS

OCT

PMMA

RGP

rigid lenses

SEBR

TMH

VAS

VVID

Wratten \#12 filter aspheric back surface contact lens geometry

back optic zone radius of a contact lens

break up time of the tear film, using fluorescein as an indication of tear film stability

observation of stained corneal epithelial cells with fluorescein (see fluorescein)

oxygen permeability (to describe the oxygen delivery through a material)

oxygen transmissibility (to describe the oxygen delivery through a material taking the thickness of the material into account)

contact lens wear on a daily basis (not overnight)

height of the lens edge relative to the corneal surface

height of the lens edge relative to the extension of the BOZR

eccentricity (amount of flattening from centre to periphery)

contact lens wear on an extended wear basis (day and night)

the food and drug administration of the USA

$\mathrm{NaFl}$ dye used to observe, assess and grade damaged corneal epithelial cells rigid gas permeable contact lens (USA)

lenses made of a PMMA material

poly-hydroxyethylmethacrylate (hydrogel contact lens material)

hydrated lenses, also referred to as soft lenses

inter eyeblink interval (time between eyeblinks)

lens is positioned between the eyelids during interblink periods

lens stays under the upper eyelid during interblink periods

keratometric value

multicurve back surface contact lens geometry

Maastricht shape topographer

non-invasive break up time of the tear film (not using fluorescein)

non-rotational symmetrical back surface contact lens geometry

ocular coherence tomography

poly-methylmethacrylate (hard contact lens material)

rigid gas permeable contact lens (UK)

term used to describe rigid gas permeable and hard lenses as a group

spontaneous eyeblink rate

tear meniscus height

visual analogue scale

visual vertical iris diameter

yellow barrier filter used to enhance the contrast of corneal fluorescein staining 



\section{Dankwoord}

De eerste conceptversies van de vraagstelling voor dit proefschrift, voor zover bewaard gebleven, dateren van vroeg in het jaar 2000. De eerste manuscripten vinden zelfs hun oorsprong in de vorige eeuw. Dat laatste klinkt uiteraard dramatischer dan het is (maar toch: 11 september 2001 was bijvoorbeeld nog een gewone datum in de agenda). In de afgelopen periode heb ik, terwijl ik aan dit project heb mogen werken, een belangrijke persoonlijke ontwikkeling doorgemaakt. Het was niet altijd gemakkelijk: integendeel soms. Maar mijn denkwereld is daardoor voorgoed veranderd.

Ik ben Professor Hendrikse bijzonder dankbaar dat hij in het najaar van 1999 meteen enthousiast reageerde op mijn voorzichtige verzoek of er misschien, wellicht, een kansje zou zijn dat ik een promotieonderzoek zou kunnen starten in Maastricht. Ik ben u, zowel als de universiteit, daarvoor eeuwig dankbaar. Dat geldt ook voor de corona: mijn dank is groot dat $\mathrm{u}$ de tijd en moeite wil nemen om $\mathrm{u}$ te verdiepen in dit proefschrift, zodat ik de laatste horde in dit proces kan nemen.

In the early process of this project it became evident that specific knowledge and expertise was desirable in order to deepen its content. I am still extremely proud and thankful that Helen Swarbrick agreed to be my external supervisor, as she was recommended to me by contact lens experts from around the world. Adding another contributing location to the existing ones truly globalized this project. But the triangle Maastricht-Washington DC-Sydney oftentimes turned out to be an effective one. By the time I was preparing papers on the east coast of the United States, based on Maastricht's guidance (while Maastricht itself was ready to go to bed), Sydney was about to wake up for additional input. E-mail conversations would sometimes continuously run around the globe (as opposed to back-andforth). Your contribution to this project has been invaluable Helen, and extends much further out than just your worldwide recognized professional knowledge. My gratitude is enormous. You really made me think. And work. But it worked. I think.

John de Brabander, mijn 'dagelijkse' begeleider (bijna letterlijk), is mijn spreekwoordelijke vader geweest in dit hele verhaal. Hij nam me vanaf het begin tot het einde aan het handje door het oerwoud van het promotieonderzoek. John: zonder jou had ik het niet gered denk ik. Bedankt voor je geduld met mijn ongeduld, en voor al de tijd en energie die je hierin hebt willen stoppen. Je bent een (Drentse) 
kei. En je was veel meer dan alleen een begeleider. En ook op veel andere vlakken zaten we vaak helemaal op één (bio)logische lijn. Het ging zelfs zo ver dat ik onderdak kreeg, te eten en te drinken. Loni en John: als jullie weer eens in Washington DC zijn, de deur staat altijd wagenwijd open.

Gedurende de laatste acht jaar heb ik samengewerkt met vele mensen aan de Universiteit van Maastricht en binnen verschillende projecten, waaronder het CLAAS project (Contact Lenses with Aspheric and Asymmetric Surfaces). Mijn dank gaat daarom uit naar medewerkers van deze projecten, en naar mijn medeauteurs van artikelen voor dit proefschrift: Rudy Nuijts, Gildas Marin, Nicolas Chateau, Pablo Artal, Norberto Lopez-Gil en Ben Lubberman. Jullie hebben een overgetelijke indruk achtergelaten op mij terwijl ik mijn eerste voorzichtige stapjes in de wereld van de wetenschap deed. Tos Berendschot, Theo Hendricks en Frans Jongsma: dank voor jullie hulp bij de statistische analyses en morele ondersteuning tijdens dit project.

James Wolffsohn and Michael Doughty: thank you for your help with subsequently the grading scale analysis and the eyeblink studies. Michael Baertschi: thanks for your clinical, and always positive, input.

De klinische studies voor dit proefschrift vonden plaats in grote contactlenspraktijken in Nederland: Groenhof Optometrie in Amstelveen en Ziemerink Totaal Optiek in Glanerbrug. Rob (Faas), Jantien (Bos), Peter (van Etten) en Jurgen (Ziemerink): dank voor jullie bijna onbegrensde gastvrijheid en hulp.

Ineke (Krijger), Yvette (Huiskes), Lisa (Starcher) and Matthew (Lampa): heel erg bedankt voor jullie hulp bij misschien wel het lastigste deel van alles: het geheel op een goede manier op papier krijgen. Ineke, ik heb veel gehad aan je hulp in de beginfase.

Special thanks to Bettie Serveert, Coldplay, Frank Boeijen, Idan Raichel, Keane, Milow, Rowwen Hèze, U2 en Starbucks Coffee for their company.

En dan zal ik daar ineens staan, voor de corona. Ik ben daarom heel blij dat ik twee mensen achter me heb staan (letterlijk) op wie ik kan bouwen: Mirjam van Tilborg en Marco van Beusekom. Mirjam en Marco: we hebben voor andere hete vuren gestaan. In de vroege jaren van de opleiding aan de Hogeschool van Utrecht, bij het podium Cornea \& Contactlenzen en het Nederlands Contactlens Congres was het ook vaak onwennig en overweldigend. En idem voor onze eerste (ook gezamelijke) optredens voor het grote publiek in binnen- en buitenland. Maar we hebben 
ons er telkens doorheen geslagen, en uiteindelijk altijd met een resultaat om trots op te zijn. Ik ben blij dat jullie er nu ook bij zijn.

Verder wil ik iedereen bedanken, van Schalkwijk tot aan Washington DC, die me direct of indirect ondersteund heeft. In Washington trof ik veel gelijkgezinden aan: gedeelde smart is halve smart. Daar heb ik veel aan gehad. Bedankt! In Schalkwijk waren er altijd wel ouders, koeien, of Bickels (in willekeurige volgorde) die me op relativerende gedachten konden brengen. Meestal was dat een welkome afwisseling. In het geval van de Bickels had het best wat genuanceerder gemogen. Pa, ma: jullie 'scholing' in doorzettingsvermogen al van jongs af aan was van doorslaggevende betekenis in dit proces. Een speciaal woord ook voor mijn schoonmoeder, die er altijd voor ons was wanneer nodig. Melanie, dank je voor je steun in het lange proces van deze promotie. Je frequente aanwezigheid in Washington en al je hulp, alswel je continue interesse, enthousiasme en morele ondersteuning hebben veel voor mij betekend.

En dan: Larissa. Je was, en bent er altijd voor me. En je hebt me altijd gestimuleerd verder te gaan. Dat was al zo toen ik naar de Hogeschool van Utrecht wilde. Of niet wilde. Maar door jou ging ik toch. En dat is zo gebleven tot aan de dag van vandaag. Ik weet heel zeker dat zonder jouw invloed ik hier niet had gestaan. Maar wat jij verder voor mij betekent, valt ver buiten de context van dit proefschrift. En dat we in de tussentijd ook nog twee kinderen hebben gekregen, blakend van Hollandse gezondheid op Amerikaanse bodem, getuigt van veel flexibiliteit van jouw kant. Luca (2004) en Youri (2006): ik kan jullie wel noemen in dit dankwoord, maar jullie hebben er nog geen benul van waar dit over gaat. Alleen dat jullie pappa en mamma nu weer even moeten missen, als pappa dit proefschrift gaat verdedigen in Maastricht. Ik kan alleen maar zeggen dat mooiere kinderen dan jullie ik me niet kan voorstellen. En als jullie me ooit zouden vragen: "Pa, wat denk je ervan als ik een promotieonderzoek zou beginnen?”, zou ik zonder enige twijfel zeggen: "doen". Het is het waard. 



\section{Curriculum Vitae}

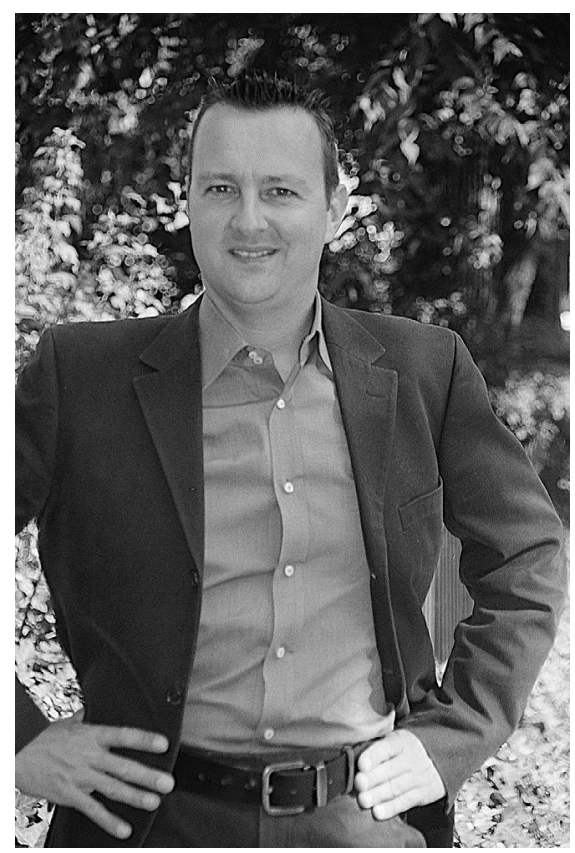

Eef van der Worp, born on August 3rd 1969 in Schalkwijk (Houten), received his optics degree in 1989 from the Christaan Huygensschool in Rotterdam. Following this he studied optometry at the Hogeschool van Utrecht. He was fortunate enough to be part of the pioneer group to study optometry in the Netherlands. During and immediately following his study, he completed educational/research programs at the University of Manchester (UK) and the University of Houston College of Optometry (Texas, USA) under supervision of Professors Nathan Efron and Jan Bergmanson, respectively. Their enthusiasm awakened his interest in the field of eye research. Eef has been in private practice part-time since 1985 and learned the skills of fitting contact lenses from Jan Merkx at his practice in Culemborg. Between 1994 and 1997, he worked part-time at the ophthalmology department of the Flevoziekenhuis in Almere.

His passion for optometry Eef could express while working at the Hogeschool van Utrecht - school of Optometry in different positions. He received his higher education didactic degree in 1995. Since 1994 he has been affiliated with the optometry school, serving as a head of the Contact Lens department for over eight years. During that period, the contact lens curriculum was formed and the eye clinic was established at the school. He currently remains a guest lecturer at the Hogeschool Utrecht, as it is called today, where he truly enjoys working with the Dutch optometrists of the future.

He has been a member of IACLE (International Association of Contact Lens Educators) since 1994 and an IACLE Fellow since 2000. Between 2003 and 2005 he was the vice-president of IACLE-Europe. He has been a Fellow of the American Academy of Optometry since 1996. He also has been active in the Dutch optometric and Contact Lens associations: between 1995 and 2000 he served as an editor 
of the Dutch contact lens \& optometry journal VISUS and between 1995 and 2005 he was the Chairman of the section Cornea \& Contact Lenses in the Netherlands, of which he is still a board member. He is a member of the Gas Permeable Lens Institute (GPLI) Advisory Committee in the USA.

Since 1993 Eef writes columns and articles for the Dutch optical journal Eyeline. As of January 2007 he produces a digital monthly contact lens newsletter for the Dutch market (CNN - Contactlens Nieuws Nederland) and is an independent consultant for the contact lens industry.

Currently Eef is involved with research projects at the University of Maastricht and at Pacific University (Oregon, USA). His specialties include corneal topography, the irregular cornea, rigid gas permeable lens wear, bifocal contact lenses and orthokeratology.

Eef is lecturing extensively at conferences and meetings worldwide and is a guest lecturer at a variety of optometry schools in the USA. He resides both in Washington DC (USA) and Amsterdam. 


\section{Publications}

\section{In this Thesis}

Van der Worp E, De Brabander J, Lubberman B, Marin G, Hendrikse F. Optimising RGP lens fitting in normal eyes using $3 D$ topography data.

Cont Lens Anterior Eye 2002;25:95-9

Van der Worp E.

Respecting the shape of the cornea in RGP lens fitting.

Optometry in Practice 2004;5:153-162

Van der Worp E, De Brabander J, Swarbrick H, Nuijts R, Hendrikse F.

Corneal desiccation in rigid contact lens wear: 3- and 9-o'clock staining - a review.

Optom Vis Sci 2003;80:280-290

Van der Worp E, De Brabander J, Swarbrick H, Hendrikse F.

Evaluation of signs and symptoms in 3- and 9-o'clock staining.

Optom Vis Sci-Accepted

Van der Worp E, De Brabander J, Swarbrick H, Hendrikse F.

Evaluation of contact lens related variables in 3-and 9-o'clock staining.

Submitted

Van der Worp E, De Brabander J, Swarbrick H, Hendrikse F.

Eyeblink frequency and type in relation to 3-and 9-o'clock staining and gas permeable contact lens variables.

Optom Vis Sci 2008;85:857-66

\section{Other Publications}

Van der Worp E, Veldman E. Glaucoomdetectie. VISUS 1994;1:9-17

Van der Worp E. Primäres Offenwinkelglaukom. Die Kontaktlinse 1997;4:31

Van der Worp E. Case report: PMMA heraanpassing/ primair open kamerhoek glaucoom. VISUS 1997;1:23-26

Van der Worp E. Tenido corneal al las tres y a las nueve ("Three and nine o'clock staining"). Ciencias de la Optometría 1998;N21-4o Trimestre 
Luiten R, Trap N, Van der Worp E, Gutter M. Geneesmiddelen en het oog. Deel I: Diagnostische pharmaca. 1999; Luiten-Barendrecht. ISBN 907625222

Van der Worp E. Adherencia de las Lentes" ("Lens adherence"). Ciencias de la Optometría 1999; N22-4o Trimestre

Van der Worp E, Morgan P. Enquête naar contactlens-aanpassingen in Nederland en Groot-Brittannië. VISUS 1999;1:9-13

Van Moerland CBJ, Van der Worp E. Invloed van de cornea-dikte op de meting van de intraoculaire druk. VISUS 1999;3:25-29

Van der Worp E, Morgan P, Efron N, Helland M, Jones L, Woods C. Contactlensaanpassingen internationaal. VISUS 2001;1:151-157

Morgan P, Efron N, Helland M, Jones L, Woods CA and van der Worp E. How does the UK market compare with other countries? Optician 2001;221(5799):26-32

Van der Worp E, Morgan P, Efron, N, Helland M, Jones D, Woods C. Contactlensaanpassingen internationaal. VISUS 2002;2:27-31

Van der Worp E, De Brabander J. De relatie tussen het draagcomfort en de passing van vormstabiele lenzen. VISUS 2002;4:116-120

Morgan P, Efron N, Woods C, Jones D, Tranoudis Y, Van der Worp E, Helland M, Chee Hong, Barr J, Bailey G. International Contact Lens Prescribing 2002. Contact Lens Spectrum 2003;1:34-8

Van der Worp E. Why the Dutch prefer GP lenses. Optometry Today 2003;7:20

Van der Worp E, De Brabander J. Oorzaken van en oplossingen voor 3 - 9 uur staining. VISUS 2003;3:69-75

Van der Worp E. Why so many GP lens wearers in the Netherlands? Global Contact 2003;No.34

De Brabander J, Chateau N, Marin G, Lopez-Gil N, Van Der Worp E, Benito A. Simulated optical performance of custom wavefront soft contact lenses for keratoconus. Optom Vis Sci 2003;9:637-43

Morgan P, Efron N, Woods C, Jones D, Tranoudis Y, Itoi M, Van der Worp E, Helland M, Yeo, Barr J. International Contact Lens Prescribing 2003. Contact Lens Spectrum 2004;1:34-7

Ruston D, Van der Worp E. Is Ortho-k OK? Optometry Today 2004;12:25-32

Morgan P, Efron N, Woods C, Jones D, Grein H-J, Tranoudis Y, Marani E, Itoi M, Van der Worp E, Phillips G, Helland M, Thunholm-Henriksson I, Hong, Barr J. International Contact Lens Prescribing 2004. Contact Lens Spectrum 2005;1:34-7

Nilsson SE, Van der Worp E. Richtlijnen voor het gebruik van contactlenzen op continuous wear basis in Europa. VISUS 2004;4: 111-114

Van der Worp, E, De Brabander, J. Modern RGP lens fitting. Optometry Today 2005;July 15:17-32

Van der Worp, E. Comfort O2 - an independent clinical evaluation. Optometry Today 2005 September 23:30-1

Van der Worp, E. The status of the RGP contact lens. Global Contact 2005;No.41:30-5 
Morgan P, Efron N, Woods C, Jones D, Tan K, Yanneth Gonzalez M, Pesinova A, Grein H, Runberg S, Tranoudis I, Chandrinos A, Fine P, Montani G, Marani E, Itoi M, Bendoriene J, Van der Worp E, Phillips G, Belousov V, Helland M, Barr J.International Contact Lens Prescribing 2005. Contact Lens Spectrum 2006;1:35-9

Van der Worp E, Ruston D. Orthokeratology: An Update. Optometry in Practice 2006: 47-60

Van der Worp E. What Contact Lenses Are Fitted Today (and Tomorrow)? Global Contact 2006;No.42:12-15

Van der Worp E. Comfort O2 RGP Material Study. Global Contact 2006;No.42:34-38

Van der Worp E. How fit is your orthokeratology patient? Contact Lens Spectrum 2006;3:21

Van der Worp E. The Art of GP Contact Lens Fitting for Presbyopia. The Boston update 2006;5:1-5

Van der Worp E. Correcting astigmatism with orthokeratology. Contact Lens Spectrum 2006;7:21

Van der Worp E. A good start (orthokeratology). Contact Lens Spectrum 2006;11:17

Morgan P, Efron N, Woods C, Jones D, Tan K, Yanneth Gonzalez M, Pesinova A, Grein H, Runberg S, Tranoudis I, Chandrinos A, Fine P, Montani G, Marani E, Itoi M, Bendoriene J, Van der Worp E, Phillips G, Belousov V, Helland M, Barr J. International Contact Lens Prescribing 2006. Contact Lens Spectrum 2007;1:34-8

Van der Worp E. Trends in contactlenzen wereldwijd. VISUS 2007;1:26-30

Van der Worp E. What is changing your market? Global Contact 2007;No.46:34-40

Van der Worp E. Keratoconus-what do we know? Contact Lens Spectrum 2007;10:36-43

Van der Worp E. Keratoconus - GKC summary. Global Contact 2007;No.47:44-47

Morgan P, Efron N, Woods C, Jones D, Tan K, Yanneth Gonzalez M, Pesinova A, Grein H, Runberg S, Tranoudis I, Chandrinos A, Fine P, Montani G, Itoi M, Bendoriene J, Van der Worp E, Phillips G, Belousov V, Helland M, Santodomingo J, Mack, C. International Contact Lens Prescribing 2007. Contact Lens Spectrum 2008;1:36-41

Van der Worp E. Orthokeratology-getting in shape. Contact Lens Spectrum 2008;3:28-33

Van der Worp E. Contact lens prescribing trends. Global Contact 2008;No.49:50-53

Van der Worp E, De Brabander J, Jongsma F. Corneal Topography. In: Bennett ES, Henry VA, eds. Clinical Manual of Contact Lenses, 3rd ed. Philadelphia: Lippincott Williams \& Wilkins, 2008:48-78 